\title{
MINOS Calibration and NA49 Hadronic Production Studies
}

\section{Robert Morse}

Submitted for the degree of D. Phil.

University of Sussex

August, 2003 


\section{Declaration}

I hereby declare that this thesis has not been submitted, either in the same or different form, to this or any other university for a degree.

Robert Morse 


\section{Acknowledgements}

This work would not have been possible without the help and support of many people:

I would like to thank my supervisor, Philip Harris, for his support, guidance and advice throughout my D.Phil. studies.

I would also like to thank the many people I have collaborated with over the years while working on MINOS: Phil Adamson, Lisa Falk Harris, Leo Jenner, Ryan Nichols, Chris Smith as well as many others, both at the calibration detector in CERN, and the far detector in Soudan. My thanks also go to the NA49 collaboration for the use of their detector to obtain the data analysed in the latter part of this thesis, and to Giles Barr for his valuable input. The time I spent working at Sussex was made very enjoyable by the people I have shared an office with: Jim Richardson, Dara Doyle, Phil Symes and Peter Smith.

Finally I would like to thank my family and friends for their support and tolerance during my long studies. Especially my parents whose encouragement and support were never-ending.

I would like to acknowledge PPARC who funded my D.Phil. .

This work is dedicated to my Granny and Grandpa Morse. 


\title{
MINOS Calibration and NA49 Hadronic
}

\section{Production Studies}

\section{Robert Morse}

Submitted for the degree of D. Phil.

University of Sussex

August, 2003

\begin{abstract}
An overview of the current status of the Main Injector Neutrino Oscillation Search (MINOS) is presented. MINOS is a long-baseline experiment with two detectors situated in North America. The near detector is based at the emission point of the NuMI beam at Fermilab, Chicago, the far detector is $735 \mathrm{~km}$ downstream in a disused iron mine in Soudan, Minnesota. A third detector, the calibration detector, is used to cross-calibrate these detectors by sampling different particle beams at CERN. A detailed description of the design and construction of the light-injection calibration system is included.

Also presented are experimental investigations into proton-carbon collisions at 158 $\mathrm{GeV} / \mathrm{c}$ carried out with the NA49 experiment at CERN. The NA49 experiment is a Time Projection Chamber (TPC) based experiment situated at CERN's North Area. It is a well established experiment with well known characteristics. The data gained from this investigation are to be used to parameterise various hadronic production processes in accelerator and atmospheric neutrino production. These hadronic production parameters will be used to improve the neutrino generation models used in calculating the neutrino oscillation parameters in MINOS.
\end{abstract}




\section{Preface}

Like many modern particle physics experiments MINOS and NA49 are both experiments that are only possible due to the large collaborations that work on them. By its nature, collaborative effort obscures the contribution of the individual. The following remarks are intended to clarify for the reader the part that I have played in the two projects.

For MINOS, my principal contribution lay in assisting with the development of the light-injection calibration system (Chapter 6) [1,2]. Although the outline concept of the system in its present form had been established by the time I joined, I carried out many crucial tests and helped with the final development of the design. These tests included, for example, verification of the PIN linearity and stability, and comparison of the PIN with a proposed alternative monitoring system that we had developed (the so-called Monitor PMT, based on scintillator doped with a radioactive source, which was eventually dropped); finalising the design of the cone and of the LIM systems (to optimise uniformity of illumination in each case); and of course establishing that the assembled system really performed as anticipated, and was able to correct gain changes to the $1 \%$ level. I was also involved early on in technical liaison with OMC, the company that built the pulser boxes, about production techniques. Much of this work was carried out during my first year. Following this, in June 2000 I gave a series of talks about the system to the MINOS collaboration; this was part of a thorough review process that led to the incorporation of our LED-based system (rather than a laser-based system that had been proposed earlier) in the MINOS detectors. 
During my third year, I spent several months at the mine in Soudan, helping to install the far detector and, in particular, helping to install and to debug the lightinjection system there. I was at the time the resident expert on the complex and sophisticated MINOS data-acquisition system, and I developed a number of software tools for data analysis and system evaluation. Likewise, I spent some months at CERN, working on the Calibration Detector during its first year of running; naturally, my principal focus was on the light-injection system, although I also found time to carry out a number of studies on the beam-related events.

MINOS will not see neutrinos from the Fermilab beam until late 2004 at the earliest. I was therefore very glad of the opportunity to continue my MINOS calibration studies in a manner that allowed me to take, and to analyse, real physics data by studying hadronic production with the NA49 experiment. As discussed in Chapter 7, the results thus obtained are important in order to understand the expected relative signals at the near and far detectors.

I took an active part in taking the NA49 data, during an intense two-week run at CERN in the summer of 2002. I spent several months thereafter looking at the data in great detail, trying to establish for myself the most appropriate cuts to make and the best approach to analysing the data. I presented the results of my workin-progress to the NA49 collaboration in October 2002. Appreciative of my effort, they brought into play the powerful tools that they had developed over many years, and produced for me the so-called micro-DSTs (Data Summary Tapes) that incorporated automatically many subtle calibration procedures and cuts, including those that I had come to realise were most important. The standard (and painstaking) NA49 fitting and analysis procedures thereafter were explained to me in some detail by Hans Gerhardt Fischer, the leader of the collaboration. Thus armed, I was able to study the reduced data set extremely carefully and effectively. I developed ROOT-based tools which enabled me to carry out this analysis on the large data set collected. This enabled the calculation of the results for hadronic production 
parameters presented in Chapter 9. 


\section{Contents}

1 Introduction 1

2 The Neutrino 3

2.1 The Discovery of the Neutrino . . . . . . . . . . . 3

2.1.1 Properties of the Neutrino . . . . . . . . . . 5

2.2 Neutrino Mass . . . . . . . . . . . . . . . 6

2.2.1 Dirac and Majorana Masses . . . . . . . . . . 7

2.2.2 The See-Saw Method . . . . . . . . . . . . . 9

2.3 Neutrino Oscillations . . . . . . . . . . . . . . . . 10

2.3.1 Two-Flavour Oscillation . . . . . . . . . . . 11

2.3.2 MSW - The Matter Effect . . . . . . . . . . . 13

3 Neutrino Oscillation Results $\quad 20$

3.1 Solar Neutrinos . . . . . . . . . . . . . . . 20

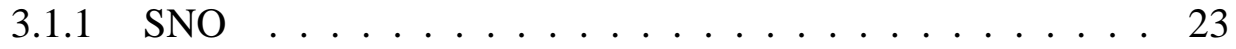

3.2 Atmospheric Neutrinos . . . . . . . . . . . . . . 26

3.2.1 Super-Kamiokande . . . . . . . . . . . . . 28

3.3 Reactor Neutrinos . . . . . . . . . . . . . . . . 28

3.3 .1 KamLAND . . . . . . . . . . . . . . 31

3.4 Accelerator Experiments . . . . . . . . . . . . . . 31

3.4.1 Short-Baseline Experiments . . . . . . . . . . 34

3.4.2 Long-Baseline Experiments . . . . . . . . . 35 
3.5 Summary of Results . . . . . . . . . . . . . . . . 35

4 MINOS Overview $\quad 38$

4.1 NuMI Beam . . . . . . . . . . . . . . . . . 40

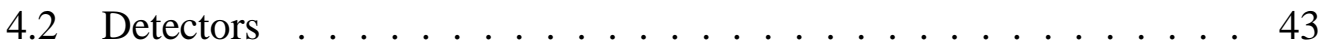

$4.2 .1 \quad$ Far Detector . . . . . . . . . . . . . 45

4.2 .2 Near Detector . . . . . . . . . . . . . . . . 48

4.2 .3 Calibration Detector .............. 52

4.3 Physics Capabilities . . . . . . . . . . . . . . . . . 54

5 Calibration $\quad 61$

5.1 Calibration Methods ................ 61

5.1 .1 Charge Injection $\ldots \ldots \ldots 62$

5.1 .2 Light Injection . . . . . . . . . . . . . . 62

5.1.3 Attenuation and Path Length Correction . . . . . . . . . 63

5.1.4 Strip-to-Strip Calibration . . . . . . . . . . . . 64

5.1 .5 Stopping Muons . . . . . . . . . . . . . 64

5.1.6 Absolute Calibration ............... 66

5.2 Calibration Detector Results . . . . . . . . . . . . . 66

5.2.1 Particle Identification and Response . . . . . . . . . . 66

6 Light Injection System $\quad 71$

6.1 Light Injection Aims . . . . . . . . . . . . . . 71

6.2 Light Injection System Design _ . . . . . . . . . . . . . 72

6.3 LEDs ............................ 75

6.4 Pulser Boxes . . . . . . . . . . . . . . . . . . 77

6.4.1 Mechanical Construction . . . . . . . . . . . . 77

6.4 .2 Cones and Collars . . . . . . . . . . . 78

6.5 Light Injection Modules _ . . . . . . . . . . . . . . . 82 
6.6 PIN Diode Linearity and Stability . . . . . . . . . . . . . 88

6.7 Trigger PMT f . . . . . . . . . . . . . . . . . . . 92

6.8 Electronics . . . . . . . . . . . . . . . 92

6.9 Linearity and the UV LED . . . . . . . . . . . . . . . . 96

7 Hadronic Production and NA49 Introduction 103

7.1 Introduction . . . . . . . . . . . . . . 103

7.1.1 MINOS Neutrino Oscillation Parameters . . . . . . . . 103

7.1.2 Atmospheric Neutrinos and the $K^{+} / \pi^{+}$Ratio . . . . . . . 108

7.2 Experimental Apparatus . . . . . . . . . . . . . . 112

7.2.1 The SPS Accelerator Beam . . . . . . . . . . . 113

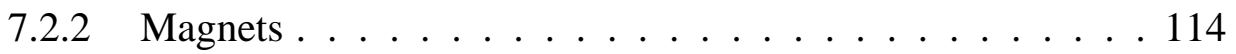

7.2.3 Time Projection Chambers . . . . . . . . . . . . . 114

7.2 .4 Electronics ................... 117

8 NA49 Data Reconstruction and Calibration $\quad 120$

8.1 Acquisition ....................... 120

8.1 .1 Storage ..................... 120

8.1.2 Reconstruction ................. 121

$8.2 \frac{d E}{d x}$ Calibration . . . . . . . . . . . . . . . . 122

8.2.1 Track Corrections . . . . . . . . . . . . . 123

8.2.2 Sector Calibration . . . . . . . . . . . . 125

8.2.3 Truncated Mean Correction _. . . . . . . . . . . . 127

8.2.4 Drift Length Correction . . . . . . . . . . . . . . 128

8.3 Particle Identification . . . . . . . . . . . . . . . . . 129

8.3.1 $\frac{d E}{d x}$ Energy Loss . . . . . . . . . . . . . . . . 129

8.3.2 Physical Data Cuts . . . . . . . . . . . . 133

$8.4 \quad \frac{d E}{d x}$ Fitting . . . . . . . . . . . . . . . . 136

8.4.1 Monte Carlo Simulation of $\frac{d E}{d x} \ldots \ldots . \ldots . . . . . . .138$ 
8.4.2 $\frac{d E}{d x}$ Fitting Corrections . . . . . . . . . . . . . 139

8.4.3 $\frac{d E}{d x}$ Fitting Method . . . . . . . . . . . . . . 140

9 NA49 - Results $\quad 144$

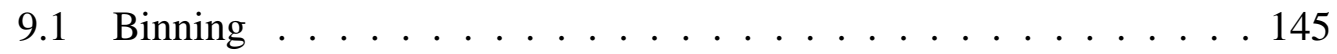

9.1.1 Bin Representation Values . . . . . . . . . . . . . . . 146

$9.1 .2 p_{T}$ Constraints . . . . . . . . . . . . . . . 148

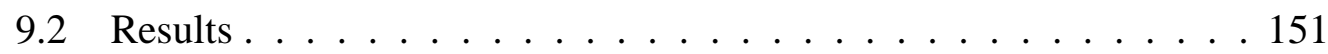

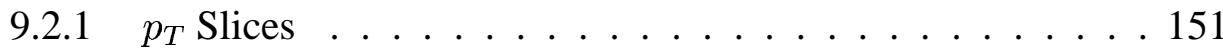

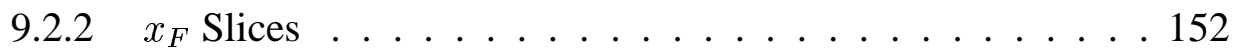

9.3 Comparison with Previous Experiments . . . . . . . . . . . 159

$\begin{array}{lr}10 \text { Conclusions } & 161\end{array}$

A NA49 $\frac{d E}{d x}$ Fitting Parameter Checking $\quad 163$

B MINOS Light Injection Software 166

$\begin{array}{lll}\text { C NA49 Software Development } & 170\end{array}$

$\begin{array}{lr}\text { Bibliography } & 173\end{array}$ 


\section{List of Figures}

2.1 Beta decay energy spectrum $\ldots \ldots \ldots \ldots \ldots$

2.2 The standard neutrino-producing double beta decay. . . . . . . . 8

2.3 Neutrino-less double beta decay $\ldots \ldots$. . . . . . . . . . 8

2.4 A schematic showing the affects $\Delta m^{2}$ and $\sin ^{2} 2 \theta$ will have on the neutrino spectra measured by MINOS. . . . . . . . . . . . . . 13

2.5 Oscillation probabilities over the MINOS baseline of $735 \mathrm{~km}$, with $\sin ^{2} 2 \theta=1 \ldots \ldots \ldots \ldots \ldots \ldots \ldots \ldots$

2.6 The neutral current interactions available to all neutrinos $\ldots \ldots$.

2.7 The charged current interactions available only to electron neutrinos 15

2.8 The distortion of $\sin ^{2} 2 \theta_{M}$ due to the MSW effect $\ldots \ldots \ldots$

2.9 The distortion of the effective $m^{2}$ differences due to the MSW effect 19

3.1 Summary of energy spectra for solar neutrinos . . . . . . . . 21

3.2 Neutrino interactions with heavy water . . . . . . . . . . . . 24

3.3 Neutrino interactions with light water $\ldots \ldots \ldots . \ldots . . \ldots 24$

3.4 Summary of recent solar neutrino experimental results $\ldots \ldots .25$

3.5 Zenith angle sensitivity in Super-Kamiokande . . . . . . . . . . 29

3.6 Summary of results for several atmospheric neutrino experiments . . 30

3.7 Super-Kamiokande zenith angle results $\ldots \ldots \ldots$

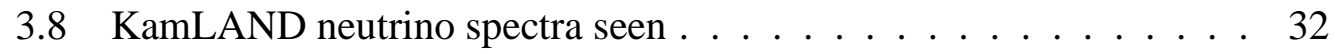

3.9 KamLAND neutrino oscillation parameter results $\ldots \ldots \ldots$

3.10 Allowed and excluded neutrino oscillation parameter space for several experimental results searching for $\nu_{\mu} \rightarrow \nu_{\tau} \ldots \ldots \ldots$ 
3.11 Allowed and excluded neutrino oscillation parameter space for several experimental results searching for $\nu_{\mu} \rightarrow \nu_{e} \ldots \ldots 37$

4.1 The route taken by the NuMI beam through the Earth . . . . . . . 39

4.2 The route taken by the NuMI beam overlaid on a map of the Great Lakes region of the USA. . . . . . . . . . . . . . . 39

4.3 Schematic of the NuMI beam line . . . . . . . . . . . . . 41

4.4 Energy spectra for the three different configurations of the NuMI beam generation equipment . . . . . . . . . . . . . 41

4.5 Schematic of the scintillator strip construction . . . . . . . . . . . 44

4.6 Schematic of the far detector . . . . . . . . . . . . 46

4.7 Magnetic field models for the near and far detectors . . . . . . . . . 47

4.8 Multiplexing at the far detector . . . . . . . . . . . . . 48

4.9 Photograph of the far detector . . . . . . . . . . . . . 49

4.10 The near detector. . . . . . . . . . . . . . . . 50

4.11 Photograph of the calibration detector . . . . . . . . . . . 52

4.12 Positioning of the calibration detector particle identification detectors 54

4.13 TOF separation at the calibration detector . . . . . . . . . 55

4.14 The three types of neutrino interaction sought by MINOS . . . . . . 56

4.15 Phase space to be covered by MINOS by the T-test for $\nu_{\mu} \rightarrow \nu_{\tau}$

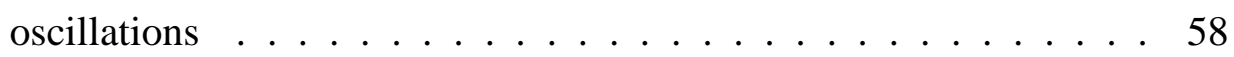

4.16 Energy spectrum produced by oscillations . . . . . . . . . . . . 59

$4.1790 \%$ exclusion limits for two years of CC spectrum oscillation analysis 60

5.1 A schematic of the calibration methods used in MINOS . . . . . . . 62

5.2 A schematic of the optical readout chain . . . . . . . . . . . 63

5.3 An example of the attenuation correction calibration . . . . . . . . 65

5.4 Calibration detector energy responses for $\pi$ and $p \ldots \ldots 7$

5.5 Hadronic energy resolution in the calibration detector . . . . . . 68 
5.6 Electron response and resolution for the calibration detector (whole momentum range $) \ldots \ldots \ldots$. . . . . . . . . . . . 69

5.7 Electron response and resolution for the calibration detector (low momentum range) . . . . . . . . . . . . . 70

6.1 A typical histogram of the spectrum of a single photoelectron peak. 72

6.2 A schematic of the light-injection system . . . . . . . . . . . 74

6.3 A demonstration of the need for uniformity in the light-injection system ........................... 76

6.4 A comparison of the wavelengths of light emitted by the blue LED and laser-stimulated MINOS scintillator. . . . . . . . . . . 77

6.5 Spectrum of light emitted from wavelength shifting fibre when excited with the light in Figure 6.4 . . . . . . . . . . . . 78

6.6 Schematic of the pulser box . . . . . . . . . . . . . 79

6.7 Photograph of a pulser box . . . . . . . . . . . . . . 80

6.8 Plot showing the beam profile of an LED used in the pulser system. 81

6.9 The "tea-strainer" plate used in the cone/collar assembly . . . . . . 82

6.10 Uniformity of illumination of a fibre tea-strainer with an LED . . . 83

6.11 Photograph of a cone and collar . . . . . . . . . . . . 84

6.12 Schematic of the cone and collar assembly . . . . . . . . . . . 84

6.13 Photograph of a scintillator module during construction . . . . . . . 85

6.14 Schematic of a Light Injection Module . . . . . . . . . . . . . . 86

6.15 LIM cutaway profile . . . . . . . . . . . . . . . 86

6.16 The LIM cover . . . . . . . . . . . . . . . . . . . . 87

6.17 Results from the testing of two LIM shapes . . . . . . . . . . . 88

6.18 Comparison of reflective surfaces of the LIM. . . . . . . . . . . . . 89

6.19 Results from the linearity tests of the PIN diodes . . . . . . . . . 90

6.20 PIN stability test results . . . . . . . . . . . . . . . . . 91 
6.21 Pulser light output shapes: widths _. . . . . . . . . . . . 93

6.22 Pulser light output shapes: heights . . . . . . . . . . . . . 93

6.23 Pulse tail elongation due to WLS fibre . . . . . . . . . . . . . 94

6.24 Schematic pulser electronic circuitry . . . . . . . . . . . . 95

6.25 Pulser non-linearity seen by the calibration detector . . . . . . . . . 97

6.26 LED spectra before and after wavelength shifting . . . . . . . . . 98

6.27 WLS absorption spectra compared with the UV pulse spectra . . . . 99

6.28 Linearity curve: UV LEDs compared to standard blue LEDs . . . 100

6.29 The residuals from linearity fits for blue and for UV LEDs . . . . . 101

6.30 Improved pulse shape from Bivar UV LEDs . . . . . . . . . . . . . 102

7.1 Predictions of absolute neutrino rates at the MINOS near detector . 104

7.2 Ratios of near/far detector neutrino rates in MINOS . . . . . . . . 105

7.3 Kaon contributions to the muon neutrino flux from the NuMI beam . 106

7.4 The distribution in $p$ and $p_{T}$ for secondary pions produced on the NuMI target . . . . . . . . . . . . . . . 107

7.5 Response for vertically upward muons in Super-Kamiokande . . . . 109

7.6 Energy distribution of the primary cosmic-ray nucleons giving rise to sub-GeV neutrino interactions in Super-Kamiokande . . . . . . . 110

7.7 Existing models of $\pi^{+}$and $K^{+}$distributions . . . . . . . . . . 111

7.8 Schematic of the NA49 detector . . . . . . . . . . . . . . . . . 112

$7.9 p, p_{T}$ phase space covered by the NA49 p-C experiment . . . . . . 113

7.10 Schematic of the NA49 TPC chambers . . . . . . . . . . . . 115

7.11 Example from the NA49 event display . . . . . . . . . . . . . 116

8.1 Multiplicity dependence of vertex fitting . . . . . . . . . . . 122

8.2 Example $\frac{d E}{d x}$ raw data plot . . . . . . . . . . . . . 123

8.3 Track length correction . . . . . . . . . . . . . . 124

8.4 The krypton calibration method for the NA49 TPCs . . . . . . . . 126 
8.5 Relative gains of NA49 TPC chambers . . . . . . . . . . . . . . 127

8.6 Example charge distribution histogram from an NA49 event . . . 128

8.7 Number of clusters per track for NA49 events . . . . . . . . . . . . 129

8.8 Drift length correction in NA49 TPCs . . . . . . . . . . . . . 130

8.9 Bethe-Bloch distribution . . . . . . . . . . . . 131

8.10 Bethe-Bloch distribution from reconstructed $\mathrm{p}+\mathrm{C}$ data . . . . . . . . 132

8.11 Schematic of the sagitta cut in NA49 event reconstruction . . . . . . 134

8.12 The relation between $\phi$ and $p$ for a range of $p_{T} \ldots \ldots . \ldots 135$

$8.1350^{\circ} \phi$ cut justification . . . . . . . . . . . . 136

8.14 Example MC $\frac{d E}{d x}$ fit . . . . . . . . . . . . . . . . 137

$8.15 \frac{d E}{d x}$ fitting algorithm. . . . . . . . . . . . . . . . . . 140

8.16 Initial fit produced by $\frac{d E}{d x}$ fitting routines . . . . . . . . . . . 141

8.17 An example of a bad fit produced by automated Bethe-Bloch shift

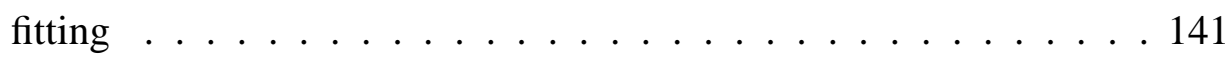

8.18 An example of a result from the $\frac{d E}{d x}$ fitting routine. . . . . . . . . . . 142

8.19 An example of a result from the $\frac{d E}{d x}$ fitting routine, showing the sum of the fits. . . . . . . . . . . . . . . . . 143

9.1 Event distribution in $x_{F}-p_{T}$ space . . . . . . . . . . . . . 145

9.2 Track distribution in relation to $x_{F} \ldots \ldots$. . . . . . . 146

9.3 The need for small $x_{F}$ bins in analysis . . . . . . . . . . . . 147

9.4 Bin representation values . . . . . . . . . . . . . . . . . . 149

9.5 NA49 momentum acceptance . . . . . . . . . . . . . . . 150

$9.6 p_{T}$ data binning . . . . . . . . . . . . . . . 151

9.7 Kaon production (as a percentage of total kaon and pion production) as a function of $x_{F}($ Feynman $x) \ldots \ldots \ldots$. . . . . . . . . . . . .

9.8 Pion production (as a percentage of total kaon and pion production) as a function of $x_{F}($ Feynman $x) \ldots \ldots \ldots$ 
$9.9 \frac{K^{+}}{\pi^{+}}$as a function of $x_{F} \ldots \ldots \ldots \ldots \ldots$

$9.10 x_{F}$ data binning . . . . . . . . . . . . . 155

9.11 Kaon production (as a percentage of total kaon and pion production) as a function of $p_{T} \ldots \ldots \ldots \ldots$

9.12 Pion production (as a percentage of total kaon and pion production) as a function of $p_{T} \ldots \ldots \ldots \ldots \ldots \ldots$

$9.13 \frac{K^{+}}{\pi^{+}}$as a function of $p_{T} \ldots \ldots . \ldots . \ldots . \ldots . \ldots 158$

9.14 Comparison of NA49 hadronic production data with SPY data . . . 159

9.15 Comparison of a selection of the NA49 hadronic production data with SPY $15 \mathrm{GeV}$ data $\ldots \ldots . . \ldots 160$

A.1 Parameter shifts for $x_{F}$ fits . . . . . . . . . . . . . . . 164

A.2 Parameter shifts for $p_{T}$ fits . . . . . . . . . . . . 165

B.1 Light Injection software screenshot (correct cabling) . . . . . . . 168

B.2 Light Injection software screenshot (incorrect cabling . . . . . . . . 169

C.1 Screenshot of NA49 analysis package . . . . . . . . . . . 171 


\section{List of Tables}

2.1 Properties of the leptons ............... 6

4.1 Summary of the MINOS parameters . . . . . . . . . . . . . 42

6.1 PIN diode specifications . . . . . . . . . . . . . . . 89

6.2 Pulser parameters . . . . . . . . . . . . . . . . 94

7.1 Properties of the TPCs . . . . . . . . . . . . . 117

7.2 Geometrical dimensions of the NA49 TPC systems. . . . . . . . . . 118 


\section{Chapter 1}

\section{Introduction}

At the heart of every important measurement lies a sound calibration. This thesis presents two very distinct studies that will each contribute in their own way towards the very precise calibration that is ultimately required for the success of the MINOS project.

The MINOS experiment comes at a very exciting time for neutrino physics. In the last five years, a flurry of activity has revealed a set of extraordinary results: the zenith-angle dependence of the atmospheric neutrino anomaly, revealed by SuperKamiokande in 1998, followed by SNO's "discovery" of the "missing" neutrinos underlying the 30-year old solar neutrino deficit, have now demonstrated beyond doubt both that neutrinos have mass and that they undergo flavour transformation. This was, to some extent, expected; the Standard Model's aesthetically unappealing assumption that neutrinos were massless was based merely on the fact that no mass had yet been observed (unsurprising, given its magnitude), together perhaps with some wishful thinking, as it made some calculations easier. Nonetheless, the demonstration of the existence of neutrino mass represents the first successful assault on the established Standard Model in its 35-year history. 
MINOS itself will be one of only three long-baseline neutrino experiments in the world (and the first of only two in the West) within the next ten years. With two main detectors separated by $735 \mathrm{~km}$, one at Fermilab in Chicago and one in North Minnesota, MINOS will be the first experiment to be able to directly compare the neutrino spectrum seen at each detector. By utilising a third detector, the calibration detector at CERN, MINOS is designed to reach a $2 \%$ relative calibration and a $5 \%$ absolute calibration. Its main aim will be to measure precisely the neutrino mixing parameters.

The mixing parameters $\Delta m^{2}$ and $\sin ^{2} 2 \theta$ arise quite simply, in principle, from studying the energy spectrum of neutrinos that arrive at the far detector. In the simplest of models, neutrino oscillations would generate a dip in the measured ratio of the number of neutrinos (as a function of energy) in the far to near detectors. The position (i.e. energy) of the dip yields $\Delta m^{2}$ directly; its magnitude gives $\sin ^{2} 2 \theta$. This is discussed in detail in Chapter 2. The energy calibration, to which the lightinjection system is central, is therefore crucial to establishing a precise measurement of $\Delta m^{2}$ in particular. However, differences in the beam itself between the two detectors can distort significantly the shape of the spectrum. It is for this reason that the hadronic production studies are also important. They provide direct input to the Monte Carlo predictions of the nature of the beam at the two sites. The information gained from the knowledge of the $\frac{K^{+}}{\pi^{+}}$ratio can also be used to improved the model used to calculate atmospheric neutrino models. 


\section{Chapter 2}

\section{The Neutrino}

\subsection{The Discovery of the Neutrino}

In the early 1930s beta-decay experiments were not producing the results that had been anticipated from energy conservation considerations. In interactions such as

$$
\mathrm{T} \rightarrow{ }^{3} \mathrm{He}+\mathrm{e}^{-}
$$

it was predicted that the electron would carry nearly all of the energy from the interaction as it is much lighter than the helium atom. However, this was not the case, as shown in figure 2.1.

In 1940 Pauli [4] suggested a solution to this missing energy problem. He proposed that there was a third particle emitted, which would share the energy with the electron, thus accounting for the spectrum seen. Fermi later formalised this idea with his model for the beta decay process, and gave the name "neutrino" to the new particle. The neutrino would then be involved in the following beta decay processes: 


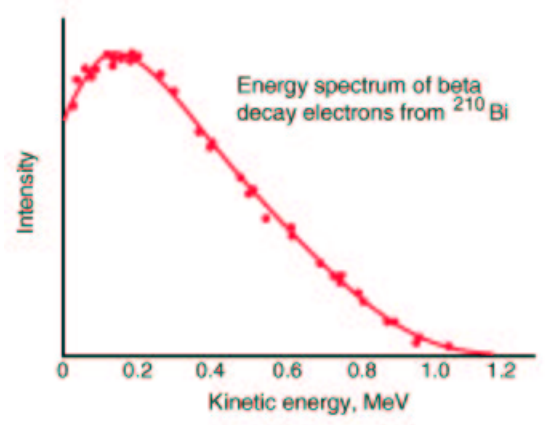

Figure 2.1: Experimental energy spectrum from beta decay. Instead of a single energy being measured, as expected, a wide range of energies was seen [3].

$$
\begin{array}{cc}
\text { Neutron beta decay } & n \rightarrow p+e^{-}+\bar{\nu} \\
\text { Electron capture } & e^{-}+p \rightarrow n+\nu \\
\text { Inverse beta decay } & \bar{\nu}+p \rightarrow n+e^{+} .
\end{array}
$$

In Fermi's theory neutrinos in their free state would be very hard to detect, as they only interact via the weak force. They would have half integer angular momentum, and zero charge. The reaction that would prove the existence of the neutrino would be inverse beta decay; this would only be possible if the neutrino existed.

However, the chances of detecting neutrinos via inverse beta decay were still very slim. There were two ways of increasing the probability of neutrino interaction. The first was to build extremely large detectors, light years long; the second was to have a far higher flux of neutrinos than was available at that time. Luckily, with the advent of nuclear fission through reactors and bombs, a source of higher flux did become available.

Reines and Cowan [5] were the first to measure a neutrino signature in 1950. They observed inverse beta decay as shown in equation 2.4. They used the Savannah 
River nuclear reactor in South Carolina as a $1 \mathrm{MeV}$ source of anti-neutrinos, with a target of cadmium chloride and water. The neutron from the decay was detected by a short burst of gamma rays from the positron, followed by a slower gamma ray pulse from the neutron capture. This was only possible due to the high neutrino flux from the reactor and the large detector volume. These are needed due to the exceptionally small cross section of the reaction: $10^{-43} \mathrm{~cm}^{2} /$ nucleon.

\subsubsection{Properties of the Neutrino}

It was demonstrated in 1956 by Wu et al. [6] that the weak interaction maximally violated parity symmetry. Their experiment was designed to measure the direction of electrons emitted from the reaction

$$
{ }^{60} \mathrm{Co}_{27} \rightarrow{ }^{60} \mathrm{~N}_{28}+\mathrm{e}^{-}+\bar{\nu}_{e}
$$

The ${ }^{60} \mathrm{Co}$ atoms were aligned with their spins in the same direction by cooling them in a strong magnetic field. The directions of the emitted electrons were then measured. Most of the electrons were emitted in the direction opposite to the nuclear spin. This parity violation suggested that only two neutrino forms, righthanded anti-neutrinos and left-handed neutrinos existed. However, for this to hold in all frames of reference, the neutrino must travel at the speed of light: otherwise, if viewed from a frame of reference sufficiently fast to overtake the neutrinos, both $\nu$ and $\bar{\nu}$ would be seen to have the opposite spin. Despite extensive searches, neutrinos of opposite spin have never been observed. It was therefore believed for many years the neutrinos travelled at $c$, and were therefore truly massless.

Further to this discovery, Goldhaber et al. [7] in 1958 demonstrated that the neutrino was left-handed. Following the discovery of the muon neutrino in 1962, measurements of the $Z^{0}$ resonance [8-13] showed that there were three types of light neutrino, accompanying the three generations of charged leptons.

In the mid 1960s, Ray Davis and his Homestake experiment [14] began to mea- 


\begin{tabular}{|c|c|c|c|}
\hline Flavour & Charge & Mass & Lifetime \\
\hline \hline $\mathrm{e}$ & -1 & $0.51 \mathrm{MeV}$ & $>4.2 \times 10^{24} \mathrm{yr}$ \\
$\nu_{e}$ & 0 & $<3 \times 10^{-3} \mathrm{eV}$ & $>21 \times 10^{9} \mathrm{~s}$ (solar), $>900 s$ reactor \\
\hline$\mu$ & -1 & $105.7 \mathrm{MeV}$ & $2.2 \times 10^{-6} \mathrm{~s}$ \\
$\nu_{\mu}$ & 0 & $<0.19 \mathrm{MeV}$ & $>2.9 \mathrm{~s}$ \\
\hline$\tau$ & -1 & $1777 \mathrm{MeV}$ & $291 \times 10^{-15} \mathrm{~s}$ \\
$\nu_{\tau}$ & 0 & $<18.2 \mathrm{MeV}$ & unknown \\
\hline
\end{tabular}

Table 2.1: Properties of the leptons. The electron neutrino mass limits are obtained from energy spectra end-points

sure the flux of neutrinos from the sun. A shortfall in the flux of neutrinos was detected, and $\sim 1 / 3$ of that expected were seen. Other later experiments looking at neutrinos generated in the sun also reported deficits.

A possible solution to this was proposed by Pontecorvo [15-17], neutrino oscillations. If the neutrinos were a superposition of mass-eigenstates, just as $\mathrm{K}^{0}$ and $\overline{\mathrm{K}^{0}}$ are in the K-system, and if they propagated as mass eigenstates but were detected as weak eigenstates, then the phenomenon of flavour oscillations could occur. This would explain the disappearance of the neutrinos, as they would have changed flavour. This theory would however require the introduction of finite neutrino mass.

\subsection{Neutrino Mass}

The standard model can be extended to incorporate neutrino masses. There are two ways of doing this, either via a Dirac mass term in the Lagrangian as is done for the charged leptons, or by introducing a Majorana mass term. 


\subsubsection{Dirac and Majorana Masses}

The Dirac mass term assumes that a particle is different from its antiparticle. For neutrinos this implies that the right-handed state $\nu_{R}$ is different from the left-handed state $\nu_{L}$. There is then a mass term which connects these two components:

$$
\begin{aligned}
\mathcal{L}_{\text {Dirac }} & =M_{\text {Dirac }} \bar{\nu} \nu \\
& =M_{\text {Dirac }}\left(\bar{\nu}_{L} \nu_{R}+\bar{\nu}_{R} \nu_{L}\right)
\end{aligned}
$$

where $\nu_{L}, \nu_{R}$ are the neutrino flavour eigenstates, and $M_{\text {Dirac }}$ is the $3 \times 3$ neutrino mass matrix.

As neutrinos are neutral particles, it is possible in principle for a neutrino to be its own anti-particle. In this case, the charge-conjugate partner of the left-handed neutrino is identical to the right-handed anti-neutrino: $\left(\nu_{L}\right)^{C}=\nu_{R} \cdot \nu=\nu_{L}+\nu_{R}^{C}$ is then a self-conjugate state, satisfying $\nu=\nu^{C}=C \bar{\nu}^{T}$ where $\mathrm{C}$ is the chargeconjugation matrix. The form of the Majorana mass term is thus

$$
\begin{aligned}
\mathcal{L} & =-\frac{1}{2} M_{\text {Majorana }} \nu \bar{\nu} \\
& =-\frac{1}{2} M_{\text {Majorana }}\left(\bar{\nu}_{L} \nu_{R}^{C}+\bar{\nu}_{R}^{C} \nu_{L}\right)
\end{aligned}
$$

These mass terms violate lepton number by two units. If the neutrino does indeed have a Majorana mass, it should be possible to observe neutrino-less double beta decay. Figure 2.2 shows the form of standard double beta decay which does produce neutrinos.

If the neutrino has Majorana mass, the right-handed anti-neutrino emitted by one decay can transform via the Majorana mass into a left-handed neutrino; this can then be absorbed by the second decay, as in figure 2.3 .

The result of this decay would be for each isotope decaying, two electrons would be produced that carried all the energy lost. In a similar fashion to figure 2.1 a delta function should be expected in the energy spectrum, rather than the continuous distribution seen when neutrinos carry away some of the energy. 


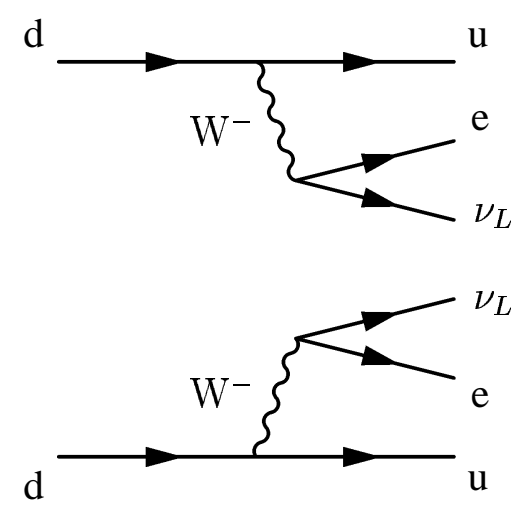

Figure 2.2: The standard neutrino-producing double beta decay.

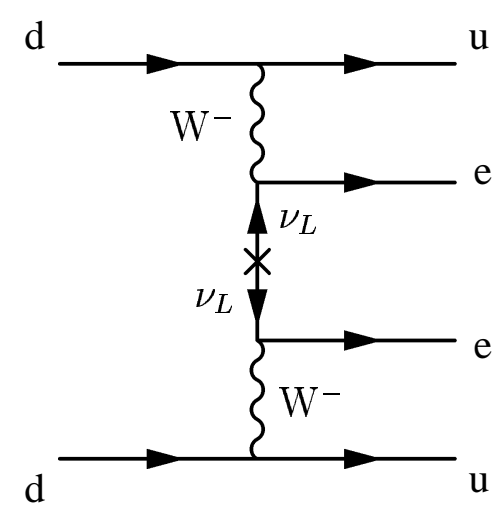

Figure 2.3: Neutrino-less double beta decay. The left-handed anti-neutrino has been transformed to become a right-handed neutrino; indicated by the cross. 
So far, the non-observation of this decay has set a limit on the electron neutrino mass of $m_{\nu_{e}} 0.5 \mathrm{eV}$ if the $\nu_{e}$ is indeed a Majorana particle [18].

\subsubsection{The See-Saw Method}

A deep mystery that remains is the major mass difference between the charged and neutral lepton masses, assuming of course that the neutrino mass is really non zero. The simplest of these theories is the "see-saw" mechanism $[19,20]$. In this method the Majorana and Dirac masses are combined.

We can write the Lagrangian for the neutrino masses as

$$
\mathcal{L}=-\frac{1}{2} \bar{n}_{L} M n_{L}+\text { h.c. }
$$

where $n_{L}=\left(\begin{array}{c}\chi \\ \omega\end{array}\right)$ and $M=\left(\begin{array}{cc}m_{L} & \frac{m^{D}}{2} \\ \frac{m^{D}}{2} & m^{R}\end{array}\right)$. Here $m_{L}$ and $m_{R}$ are the Majorana masses associated with the left-handed and right-handed neutrinos, and $m^{D}$ is the Dirac mass. The two self conjugate fields

$$
\begin{aligned}
& \chi=\nu_{L}+\left(\nu_{L}\right)^{C} \\
& \omega=\nu_{R}+\left(\nu_{R}\right)^{C}
\end{aligned}
$$

describe the left and right handed neutrinos.

In the general case of $n_{a}$ active neutrinos, and $n_{s}$ sterile neutrinos, $\mathrm{M}$ is an $\left(n_{a}+n_{s}\right) \times\left(n_{a}+n_{s}\right)$ matrix. When assuming only two neutrino generations, the diagonalisation of $\mathrm{M}$ results in the mass eigenvalues

$$
m_{1,2}=\frac{1}{2}\left[\left(m_{L}^{M}+m_{R}^{M}\right) \pm \sqrt{\left(m_{L}^{M}-m_{R}^{M}\right)^{2}+4\left(m^{D}\right)^{2}}\right]
$$

There are then four possible cases to be considered for these masses: 


$$
\begin{array}{ll}
m_{L}^{M}=m_{R}^{M}=0 \rightarrow m_{1,2}=m^{D} & - \text { The result is a pure Dirac neutrino field. } \\
m^{D} \gg m_{L}^{M}, m_{R}^{M} \rightarrow m_{1,2} \approx m^{D} & - \text { The neutrinos are pseudo-Dirac neutrinos. } \\
m^{D}=0 \rightarrow m_{1,2}=m_{L}^{M}, m_{R}^{M} & - \text { The neutrinos are pure Majorana particles. } \\
m_{R}^{M} \gg m^{D}, m_{L}^{M}=0 & - \text { This leads to the see-saw mechanism. }
\end{array}
$$

In the see-saw scenario there are two neutrino masses produced, one of which is a very light state $m_{1}$ and the other of which if a very heavy state $m_{2}$ :

$$
\begin{aligned}
& m_{1} \approx \frac{m^{D}}{m_{R}^{M}} \ll m^{D} \\
& m_{2} \approx m_{R}^{M} .
\end{aligned}
$$

\subsection{Neutrino Oscillations}

If neutrinos do indeed have mass, and flavour mixing does occur, then the flavour eigenstates can be written as a linear superposition of the mass eigenstates:

$$
\left|\nu_{l}\right\rangle=\sum_{m} U_{l m}\left|\nu_{m}\right\rangle
$$

where $l$ indicates the flavour eigenstates $\nu_{e}, \nu_{\mu}$ and $\nu_{\tau}$, and $m$ indicates the three mass eigenstates $\nu_{1}, \nu_{2}$ and $\nu_{3}$. $U$ represents a matrix known as the Maki-NakagawaSakata (MNS) leptonic mixing matrix [21], which can be parametrised as:

$$
\left(\begin{array}{c}
\nu_{e} \\
\nu_{\mu} \\
\nu_{\tau}
\end{array}\right)=\left(\begin{array}{ccc}
c_{1} & s_{1} c_{3} & s_{1} s_{3} \\
-s_{1} c_{2} & c_{1} c_{2} c_{3}-s_{2} s_{3} e^{i \delta} & c_{1} c_{2} s_{3}+s_{2} c_{3} e^{i \delta} \\
-s_{1} s_{2} & c_{1} s_{2} s_{3}+c_{2} s_{3} e^{i \delta} & c_{1} s_{2} s_{3}-c_{2} c_{3} e^{i \delta}
\end{array}\right)\left(\begin{array}{c}
\nu_{1} \\
\nu_{2} \\
\nu_{3}
\end{array}\right)
$$

where $c_{i} \equiv \cos \theta_{i}$ and $s_{i} \equiv \sin \theta_{i}$. The $\theta_{i}$ are the weak mixing angles, and $e^{i \delta}$ is a complex phase.

We can then write, for an initial state $\nu_{\alpha}$ at $t=0$,

$$
\left|\nu_{\alpha}(0)\right\rangle=U_{\alpha i}\left|\nu_{i}\right\rangle
$$

where the Einstein summation convention is implied. The neutrino will then propagate as

$$
\left|\nu_{\alpha}(t)\right\rangle=U_{\alpha i} e^{-i E_{i} t}\left|\nu_{i}\right\rangle,
$$


with the neutrinos having momentum $p$ and masses $m_{i}$. $E_{i}$ for the mass eigenstates are therefore given by $E_{i}^{2}=p^{2}+m_{i}^{2}$.

Therefore, the probability of finding the neutrino in state $\nu_{\beta}$ at time $t$ is

$$
\begin{aligned}
P_{\alpha \rightarrow \beta} & =\left|\left\langle\nu_{\beta} \mid \nu_{\alpha}(t)\right\rangle\right|^{2} \\
& =\left|\left\langle U_{\beta i} \nu_{i} \mid U_{\alpha i} e^{-i E_{i} t} \nu_{i}\right\rangle\right|^{2} \\
& =\sum_{i}\left|U_{\alpha i}\right|^{2}\left|U_{\beta i}\right|^{2}+\sum_{i \neq j} U_{\alpha i} U_{\beta i}^{*} U_{\alpha j}^{*} U_{\beta j} e^{-i\left(E_{i}-e_{j}\right) t} .
\end{aligned}
$$

\subsubsection{Two-Flavour Oscillation}

By considering a simplified case of mixing, using only two neutrino masses, we can discover how the phenomenon of neutrino oscillation depends on the neutrino mass differences. This approach is taken as many theoretical models predict that the mixing angle $\theta_{13}$ is very small, leaving the mixing between only two flavours being dominant.

By expressing the two neutrino flavours $\nu_{e}$ and $\nu_{\mu}$ as mixtures of two mass eigenstates $\nu_{1}$ and $\nu_{2}$ with masses $m_{1}$ and $m_{2}$, as with the three-generation model, we can write

$$
\left(\begin{array}{c}
\left|\nu_{e}\right\rangle \\
\left|\nu_{\mu}\right\rangle
\end{array}\right)=\left(\begin{array}{cc}
\cos \theta & \sin \theta \\
-\sin \theta & \cos \theta
\end{array}\right)\left(\begin{array}{c}
\left|\nu_{1}\right\rangle \\
\left|\nu_{2}\right\rangle
\end{array}\right) .
$$

We can then define the two neutrino wave functions at time $t=0$ as

$$
\begin{aligned}
& \left|\nu_{e}(0)\right\rangle=\left|\nu_{e}\right\rangle=\sin \theta\left|\nu_{2}\right\rangle+\cos \theta\left|\nu_{1}\right\rangle \\
& \left|\nu_{\mu}(0)\right\rangle=\left|\nu_{\mu}\right\rangle=-\sin \theta\left|\nu_{1}\right\rangle+\cos \theta\left|\nu_{2}\right\rangle
\end{aligned}
$$

where $\theta$ represents the mixing angle between the two mass eigenstates. The wave functions propagate, so that at time $t$ we have

$$
\left|\nu_{\mu}(t)\right\rangle=-e^{-i E_{1} t} \sin \theta\left|\nu_{1}\right\rangle+e^{-i E_{2} t} \cos \theta\left|\nu_{2}\right\rangle,
$$


with a similar expression for $\nu_{e} . E_{1}$ and $E_{2}$ are the energies of the states $\left|\nu_{1}\right\rangle$ and $\left|\nu_{2}\right\rangle$ respectively, and, as $E_{\nu}>>m_{\nu}$, we can approximate

$$
\begin{aligned}
E_{\nu} & =\left(p_{\nu}^{2}+m_{\nu}^{2}\right)^{1 / 2} \\
& \approx p_{\nu}+\frac{m_{\nu}^{2}}{2 p_{\nu}} \\
\therefore E_{1}-E_{2} & \approx \frac{m_{\nu_{1}}^{2}-m_{\nu_{2}}^{2}}{2 p_{\nu}} \\
& \approx \frac{\Delta m_{\nu_{1} \nu_{2}}^{2}}{2 p_{\nu}} .
\end{aligned}
$$

The wave function (2.26) then becomes

$$
\left|\nu_{\mu}(t)\right\rangle=e^{-i t\left(p+\frac{m_{\nu_{1}}^{2}}{2 E_{\nu}}\right)}\left(-\sin \theta\left|\nu_{1}\right\rangle+\cos \theta\left|\nu_{2}\right\rangle e^{\frac{i t \Delta m_{\nu_{1} \nu_{2}}^{2}}{2 E_{\nu}}}\right) .
$$

We can now calculate the probability of oscillation $P\left(\nu_{\mu} \rightarrow \nu_{e}\right)$ by replacing $t$ with $x / c$, as neutrinos travel at almost the speed of light.

$$
\begin{aligned}
P\left(\nu_{\mu} \rightarrow \nu_{e}\right) & =\left|\left\langle\nu_{e} \mid \nu_{\mu}\right\rangle\right|^{2} \\
\left|\left\langle\nu_{e} \mid \nu_{\mu}\right\rangle\right|^{2} & =\left|\cos \theta \sin \theta\left(1-e^{\frac{i \Delta m^{2} t}{2 E_{\nu}}}\right)\right|^{2} \\
& =\sin ^{2} 2 \theta \sin ^{2}\left(\frac{\Delta m^{2} x}{4 E_{\nu}}\right)
\end{aligned}
$$

in natural units. By quoting $\Delta m^{2}$ in $\mathrm{eV}^{2}, x$ in $\mathrm{km}$, and $E_{\nu}$ in $\mathrm{GeV}^{2}$, we derive the very popular oscillation appearance formula

$$
P\left(\nu_{\mu} \rightarrow \nu_{e}\right)=\sin ^{2} 2 \theta \sin ^{2}\left(\frac{1.27 \Delta m^{2} x}{E_{\nu}}\right)
$$

and its partnering disappearance formula

$$
P\left(\nu_{\mu} \rightarrow \nu_{\mu}\right)=1-\sin ^{2} 2 \theta \sin ^{2}\left(\frac{1.27 \Delta m^{2} x}{E_{\nu}}\right)
$$

The two unknown parameters of these formula are $\Delta m^{2}$ and $\sin ^{2} 2 \theta$. When looking for neutrino oscillations, $\sin ^{2} 2 \theta$ determines the maximum possibility of oscillation, 
and therefore the depth of any dip (or peak in $\nu$ appearance experiments) in a neutrino spectrum. Accurate measurement of this parameter needs a large number of statistics. $\Delta m^{2}$ however, defines the position of any dip (or peak) in the neutrino spectrum, an accurate measurement of this requires good energy calibration. The affect of these properties on the neutrino spectra measured by MINOS is demonstrated in figure 2.4

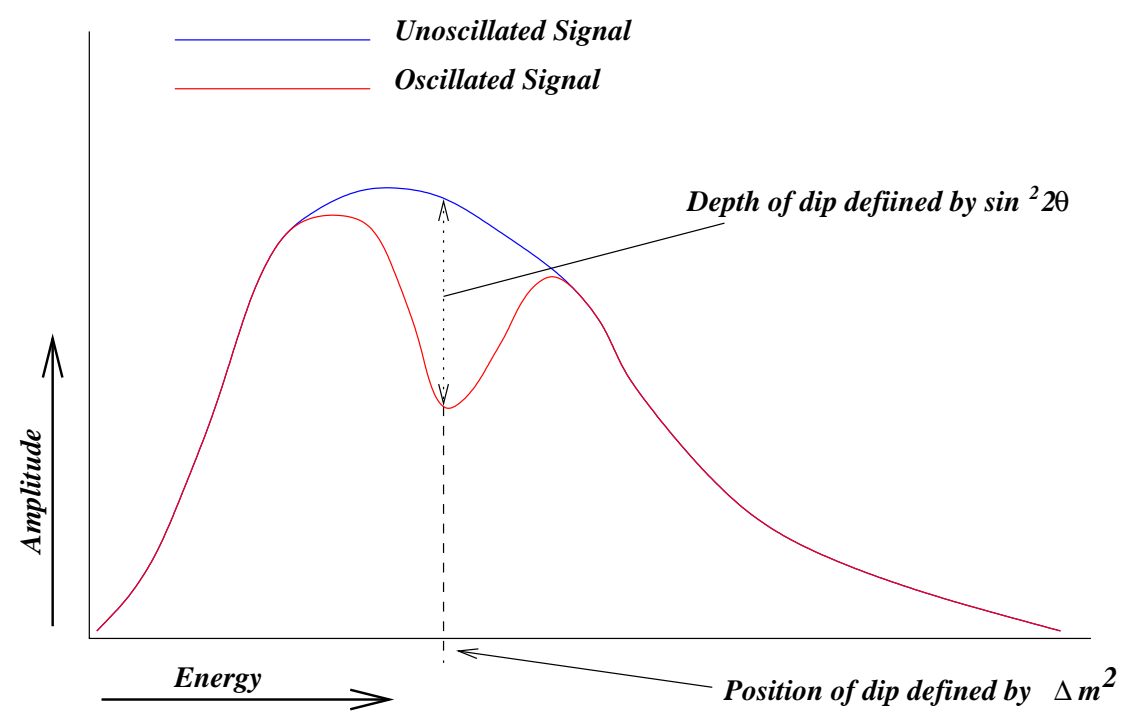

Figure 2.4: A schematic showing the affects $\Delta m^{2}$ and $\sin ^{2} 2 \theta$ will have on the neutrino spectra measured by MINOS.

Figure 2.5 shows the oscillation probability for a baseline of $735 \mathrm{~km}$, which is that of MINOS, and $\sin ^{2} 2 \theta=1$, for several neutrino energies.

\subsubsection{MSW - The Matter Effect}

Neutrinos can interact with matter, albeit with very small cross sections $\left(\sim 10^{-42}-\right.$ $10^{-43} \mathrm{~m}^{2}$ ). They can take part in elastic forward scattering, which leaves their momentum unchanged. This takes place via the neutral current interactions shown in figure 2.6. Electron neutrinos can also take part in the charged current interaction in figure 2.7. The effect that this would have on neutrino oscillations was first 

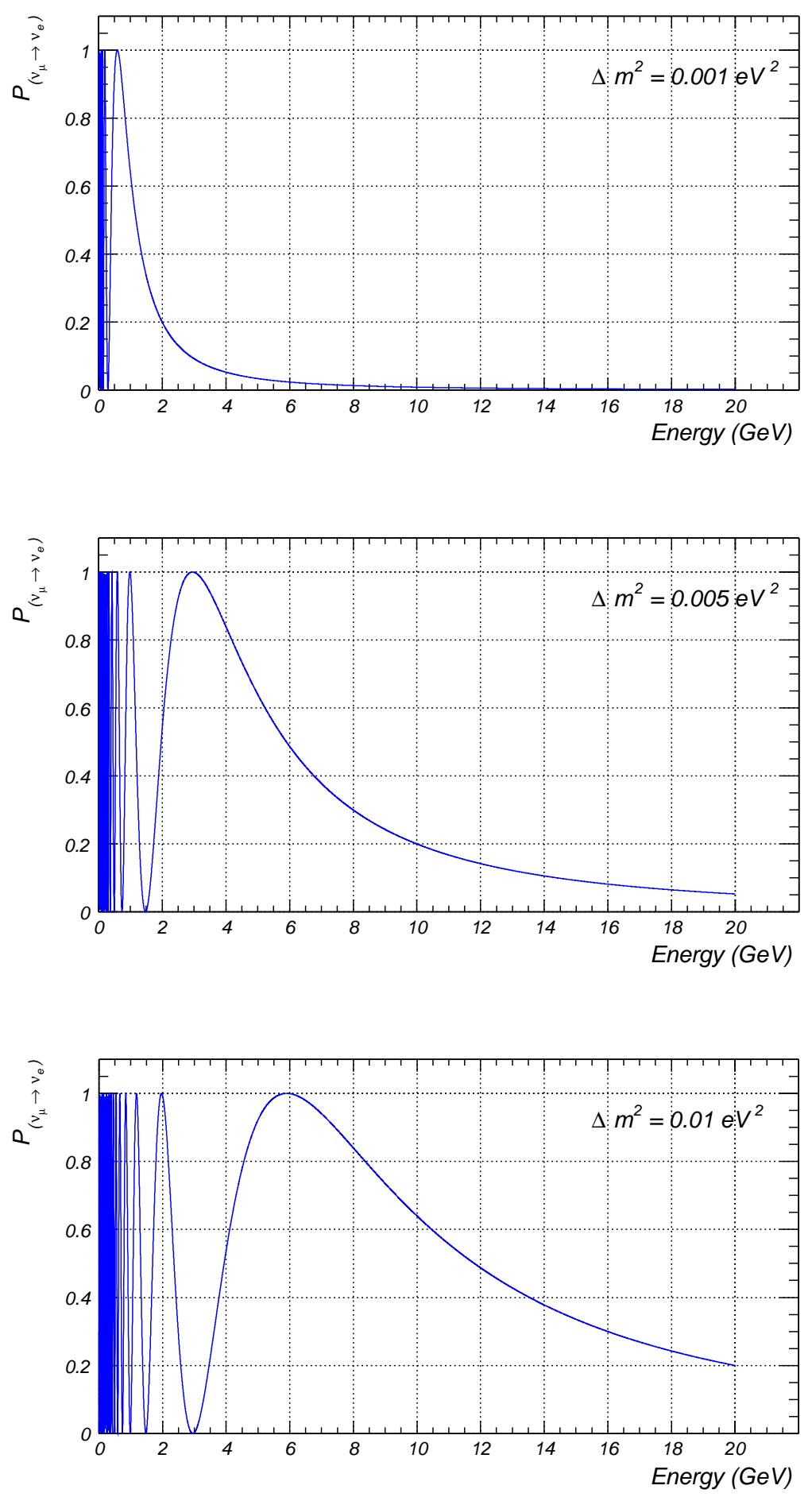

Figure 2.5: Oscillation probabilities over the MINOS baseline of $735 \mathrm{~km}$, with $\sin ^{2} 2 \theta=1$. 
studied by Wolfenstein [22] and independently by Mikheyev and Smirnov [23], and is known as the MSW effect.

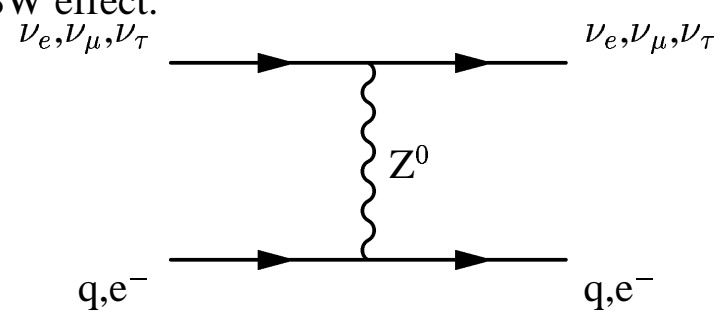

Figure 2.6: The neutral current interactions available to all neutrinos

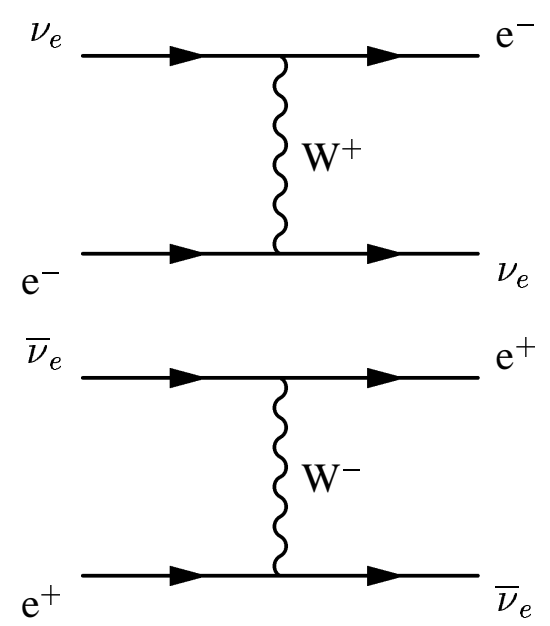

Figure 2.7: The charged current interactions available only to electron neutrinos

As indicated above the effect arises due to the charged current interaction available only to the electron neutrino. As the neutral current interaction is available to all neutrinos, its contribution to the different flavours is the same, and it therefore only adds an overall phase. The result of the charged interaction is dependent on the electron number density of the material, and for two flavour mixing is given by

$$
\begin{array}{r}
V_{C C}\left(\nu_{e}\right)=\sqrt{2} G_{F} N_{e} \\
V_{C C}\left(\bar{\nu}_{e}\right)=-\sqrt{2} G_{F} N_{e} .
\end{array}
$$

The evolution equation in free space is given in terms of flavour by

$$
i \frac{d}{d t}\left(\begin{array}{c}
\nu_{e} \\
\nu_{\mu}
\end{array}\right)=\left(\begin{array}{cc}
E_{1} \cos ^{2} \theta+E^{2} \sin ^{2} \theta & \left(E_{2}-E_{1}\right) \sin \theta \cos \theta \\
\left(E_{2}-E_{1}\right) \sin \theta \cos \theta & -E_{1} \sin ^{2} \theta+E_{2} \cos ^{2} \theta
\end{array}\right)\left(\begin{array}{c}
\nu_{e} \\
\nu_{\mu}
\end{array}\right) .
$$


By adding in the matter effects contributed by the electron interactions in equation 2.37, we can write

$i \frac{d}{d t}\left(\begin{array}{c}\nu_{e} \\ \nu_{\mu}\end{array}\right)=\left(\begin{array}{cc}\left(E_{2}-E_{1}\right) \sin ^{2} \theta+\sqrt{2} G_{F} N_{e} & \left(E_{2}-E_{1}\right) \sin \theta \cos \theta \\ \left(E_{2}-E_{1}\right) \sin \theta \cos \theta & \left(E_{2}-E_{1}\right) \cos ^{2} \theta\end{array}\right)\left(\begin{array}{c}\nu_{e} \\ \nu_{\mu}\end{array}\right)$.

In this step, $E_{1}$ times the unit matrix has been subtracted from the Hamiltonian. This is equivalent to removing an overall phase, and it therefore has no effect on the neutrino oscillations, which are a consequence of phase differences.

The eigenstates are then

$$
\begin{aligned}
& \left|\nu_{1}^{\prime}\right\rangle=\cos \theta_{M}\left|\nu_{e}\right\rangle+\sin \theta_{M}\left|\nu_{\mu}\right\rangle \\
& \left|\nu_{2}^{\prime}\right\rangle=-\sin \theta_{M}\left|\nu_{e}\right\rangle+\cos \theta_{M}\left|\nu_{\mu}\right\rangle
\end{aligned}
$$

where the mixing angle in matter $\theta_{M}$ is

$$
\tan 2 \theta_{M}=\frac{\left(E_{2}-E_{1}\right) \sin 2 \theta}{\left(E_{2}-E_{1}\right) \cos 2 \theta-\sqrt{2} G_{F} N_{e}} .
$$

We can then recover the derivation of oscillation in free space, and include the matter effects, by writing

$$
\begin{aligned}
P_{\left(\nu_{\mu} \rightarrow \nu_{e}\right)} & =\left|\left\langle\nu_{e} \mid \nu_{\mu}\right\rangle\right|^{2} \\
& =\sin ^{2} 2 \theta \sin ^{2}\left(\frac{\left(E_{1}{ }^{\prime}-E_{2}{ }^{\prime}\right) L}{2}\right)
\end{aligned}
$$

where $E_{1}{ }^{\prime}$ and $E_{2}{ }^{\prime}$ are the eigenvalues of equations 2.41 and 2.42. The difference between these can be shown to be

$$
E_{1}{ }^{\prime}-E_{2}{ }^{\prime}=\frac{\Delta m_{21}^{2}}{2 E} \sqrt{\sin ^{2} 2 \theta+\left(\cos 2 \theta-\frac{\sqrt{2} G_{F} N_{e}}{\Delta m_{21}^{2} / 2 E}\right)^{2}} .
$$

This therefore results in an expression similar to that for free space oscillations:

$$
P_{\left(\nu_{\mu} \rightarrow \nu_{e}\right)}=\sin ^{2} 2 \theta_{M} \sin ^{2}\left(\frac{1.27 \Delta M^{2} L}{E}\right)
$$


with

$$
\sin ^{2} 2 \theta_{M}=\frac{\sin ^{2} 2 \theta}{\sin ^{2} 2 \theta+\left(\cos 2 \theta-\frac{\sqrt{2} G_{F} N_{e}}{\Delta m_{21}^{2} / 2 E}\right)^{2}},
$$

and

$$
\Delta M^{2}=\Delta m_{21}^{2} \sqrt{\sin ^{2} 2 \theta+\left(\frac{-\sqrt{2} G_{F} N_{e}}{\Delta m_{21}^{2} / 2 E}+\cos 2 \theta\right)^{2}} .
$$

It can be seen that at low matter densities, $N_{e} \rightarrow 0$, this reverts to the vacuum solution for oscillations. However, at high matter densities $\sin ^{2} \theta_{M} \propto 1 / N_{e}^{2}$, so the oscillation amplitude is suppressed. This MSW effect allows maximal mixing even for low $\sin ^{2} 2 \theta$, since $\sin ^{2} 2 \theta_{M}$ equals one if

$$
\frac{\sqrt{2} G_{F} N_{e}}{\Delta m_{21}^{2} / 2 E}=\cos 2 \theta .
$$

This allows enhancement of neutrino oscillations if $m_{2}>m_{1}$ and anti-neutrino oscillations if $m_{2}<m_{1}$. Figures 2.8 and 2.9 show the distortion of the oscillation parameters due to the MSW effect. 
$\sin ^{2} 2 \theta=1$

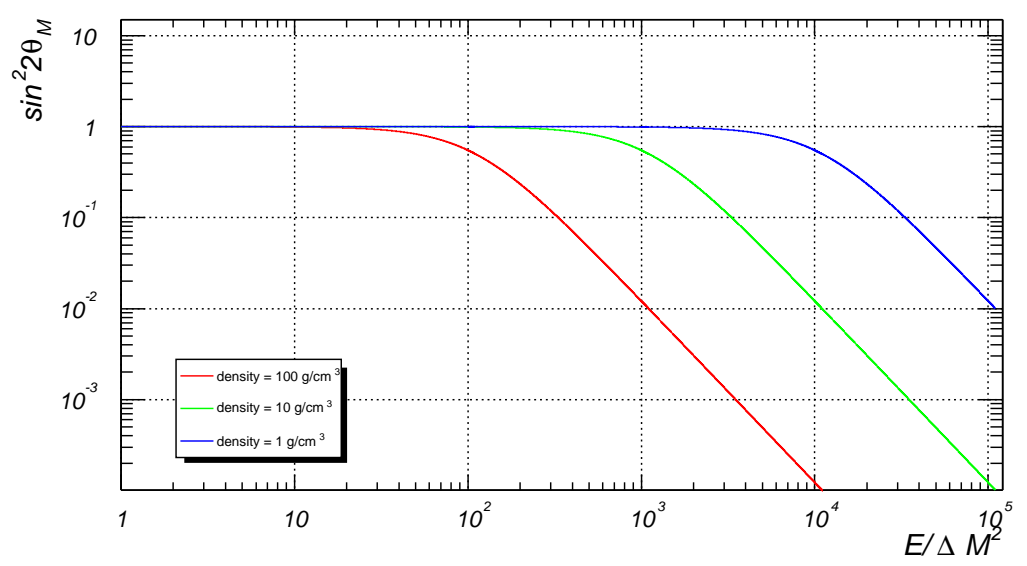

$\sin ^{2} 2 \theta=0.1$

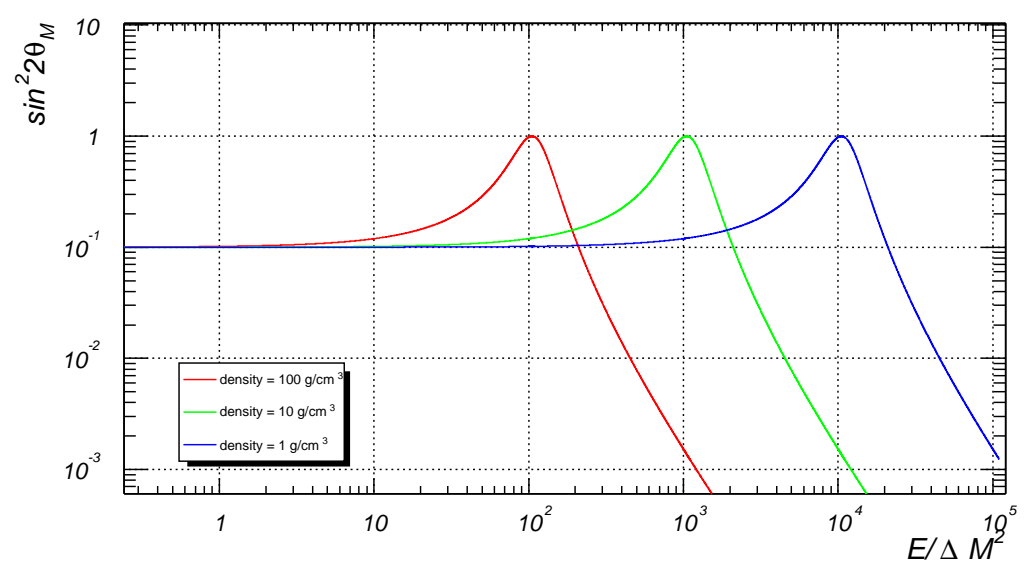

$\sin ^{2} 2 \theta=0.01$

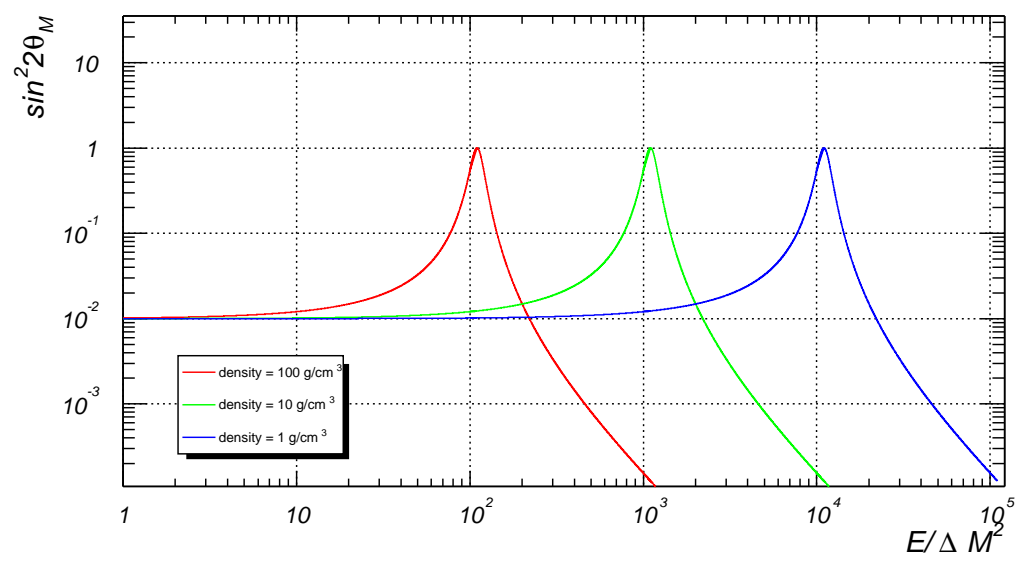

Figure 2.8: The distortion of $\sin ^{2} 2 \theta_{M}$ due to the MSW effect; note the possibility of $\sin ^{2} 2 \theta_{M}=1$ even though $\sin ^{2} 2 \theta<1$. 

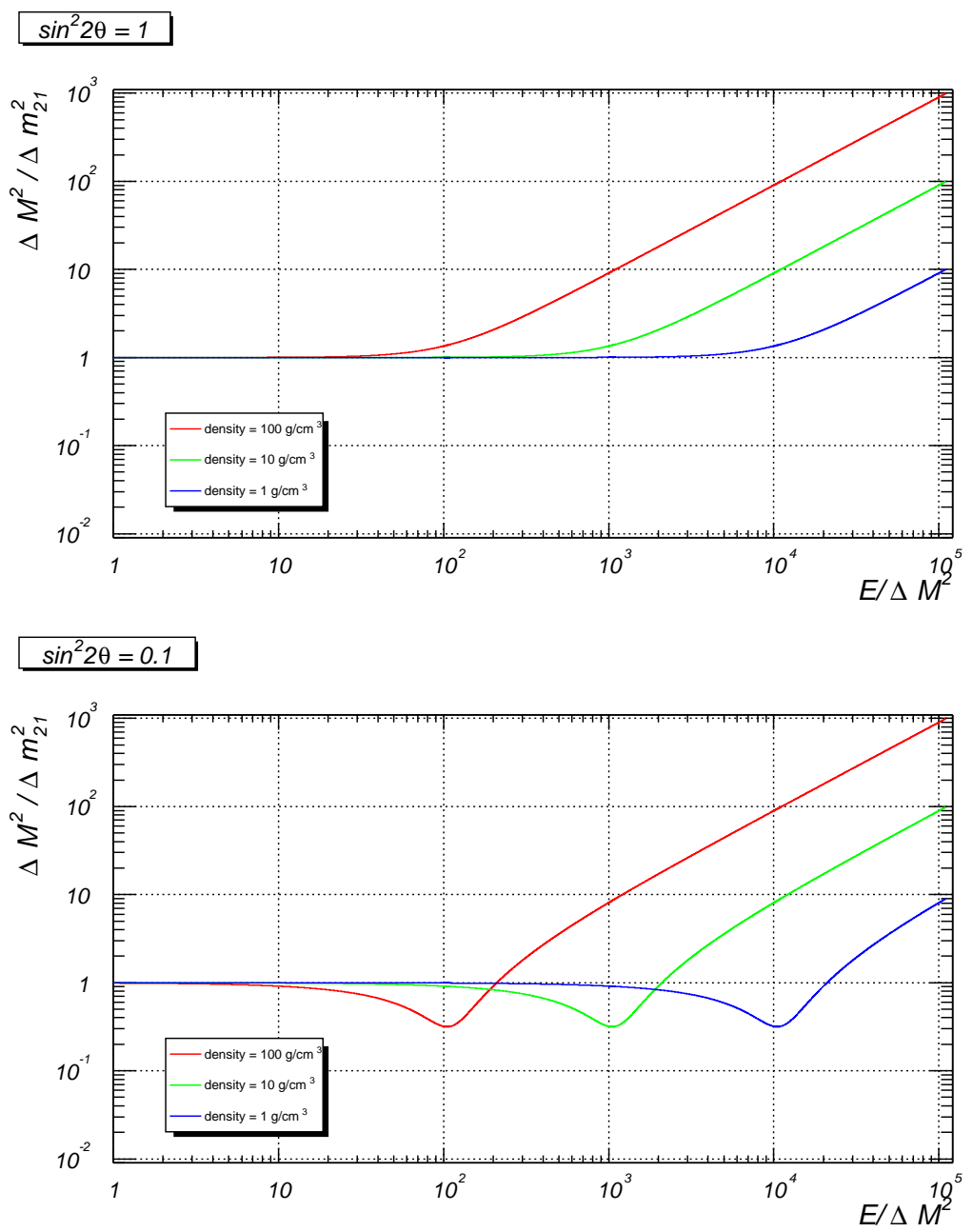

$\sin ^{2} 2 \theta=0.01$

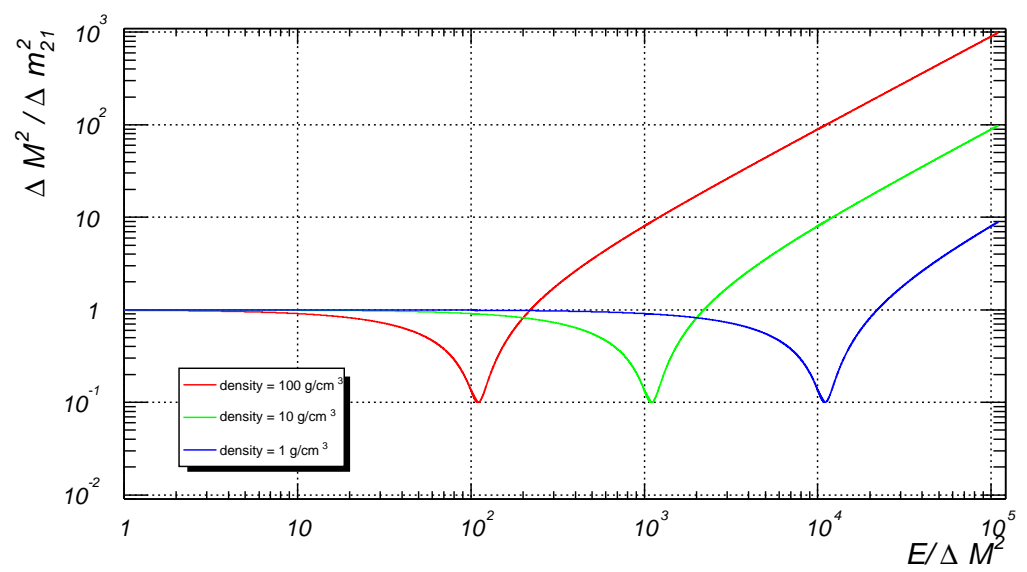

Figure 2.9: The distortion of the effective $m^{2}$ differences due to the MSW effect. 


\section{Chapter 3}

\section{Neutrino Oscillation Results}

Since the first discovery of the neutrino, there have been many experiments to investigate its properties. Many of these have attempted to detect neutrino oscillations. This chapter sets out to summarise the more influential neutrino oscillation experiments. Neutrino experiments may be grouped into four categories: Solar, Atmospheric, Reactor and Accelerator.

\subsection{Solar Neutrinos}

According to modern astrophysical models there are three main sources of neutrinos from the nuclear reactions within the sun:

- The proton-proton produced neutrinos are the most abundant, and have a continuous energy spectrum up to about $420 \mathrm{KeV}$.

$$
p+p \rightarrow{ }^{2} \mathrm{H}+\mathrm{e}^{+}+\nu_{e}
$$

- ${ }^{7} \mathrm{Be}$ decay produces $\nu_{e}$ with energies at $862 \mathrm{Kev}$ and $383 \mathrm{Kev}$, with a total flux of about $8 \%$ that of the proton-proton production: 


$$
{ }^{7} \mathrm{Be}+e^{-} \rightarrow{ }^{7} \mathrm{Li}\left({ }^{7} \mathrm{Li}^{*}\right)+\nu_{e}
$$

- ${ }^{8}$ Be decay produces about $10^{-4}$ times the flux of the proton-proton reactions, but produces the most energetic neutrinos with energies reaching $14 \mathrm{MeV}$ :

$$
{ }^{8} \mathrm{Be} \rightarrow{ }^{8} \mathrm{Be}^{*}+\mathrm{e}^{+}+\nu_{e} .
$$

Figure 3.1 shows the energy spectrum of neutrinos from various solar models along with the fluxes. It also shows the energy coverage of various detector technologies.

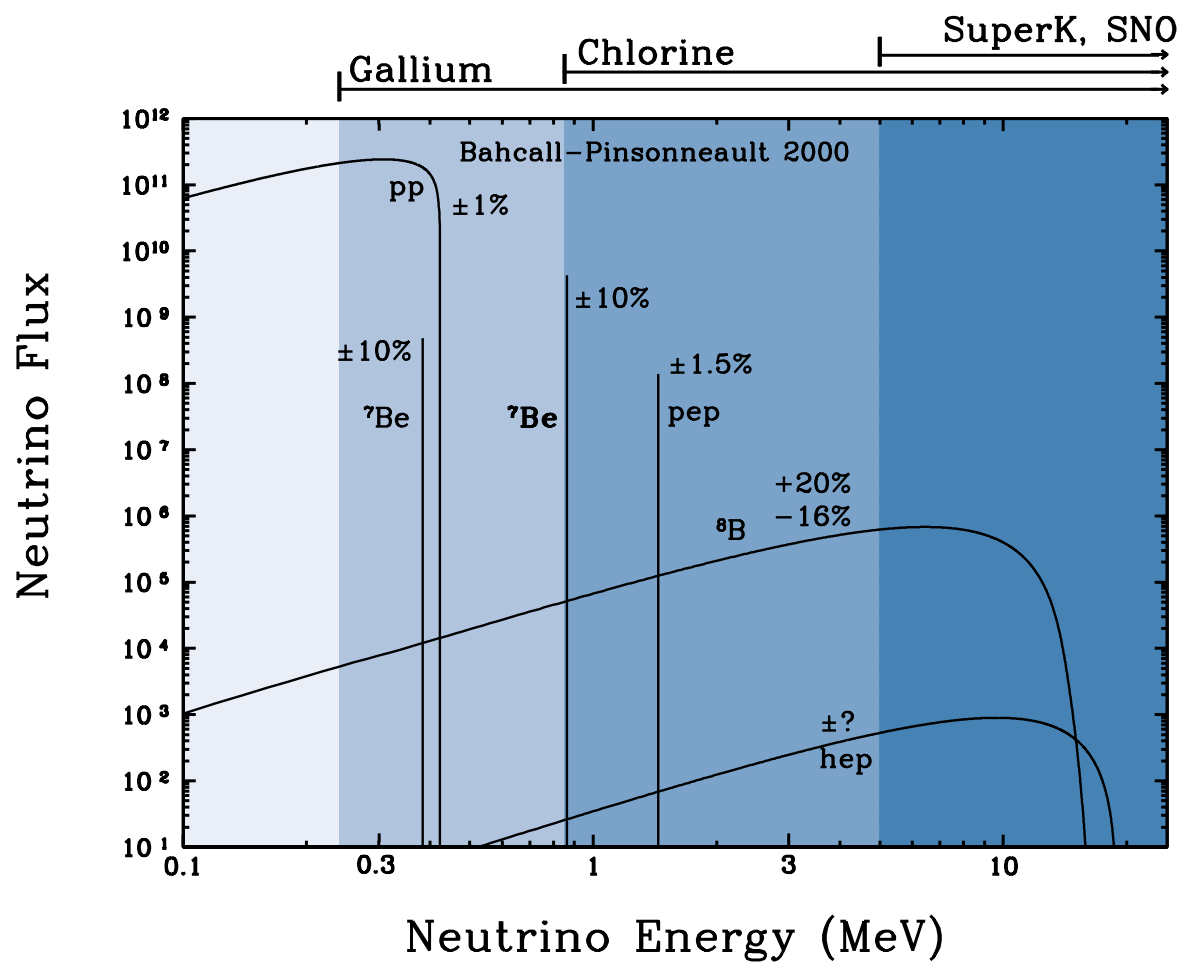

Figure 3.1: The energy spectrum for solar neutrinos, and the thresholds of various neutrino detector technologies [24].

In 1960 Ray Davis [14] began developing techniques to measure the neutrino fluxes from the sun. His pioneering experiment was in the Homestake mine, and 
was based on the following reaction:

$$
\nu_{e}+{ }^{37} \mathrm{Cl} \rightarrow \mathrm{e}^{-}+{ }^{37} \mathrm{Ar}
$$

This reaction has a threshold neutrino energy of $814 \mathrm{KeV} .100,000$ gallons of perchloroethylne was used as the chlorine source. After several weeks of running the chlorine was filtered to remove the argon. The decay rate of the argon was then monitored to reveal the number of $\nu_{e}$ that had interacted. Due to the high threshold energy, this experiment was only sensitive to the ${ }^{7} \mathrm{Be}$ and ${ }^{8} \mathrm{Be}$ sources from 3.2 and 3.3. The experiment recorded a deficit in the number of neutrinos detected, suggesting either that oscillations were taking place or that there were problems with the Standard Solar Model.

These early results have been confirmed by later experiments. The SAGE [25, 26] and GALLEX/GNO [27,28] experiments employed a different radiochemical process to try to measure the solar neutrino flux. These experiments used gallium rather than chlorine:

$$
\nu_{e}+{ }^{71} \mathrm{Ga} \rightarrow \mathrm{e}^{-}+{ }^{71} \mathrm{Ge}
$$

This reaction has a far lower threshold - just $233 \mathrm{KeV}$ - than reaction 3.4. It is therefore sensitive to the lower energy neutrinos produced from the $\mathrm{p}+\mathrm{p}$ reaction (3.1). In a similar manner to the Homestake experiment, after several weeks of exposure the gallium was filtered and the germanium was counted to reveal the number of $\nu_{e}$ that had interacted.

Neither of these experiments measured the neutrino flux in real time. They both integrated the neutrinos counted over a long period of time, and gave an average for the period.

A real-time neutrino counter was produced by the Kamiokande $[29,30]$ experiment and its larger successor Super-Kamiokande $[31,32]$. They were both pure- 
water C̆erenkov counters based $1 \mathrm{~km}$ underground in the Kamioka mine in Japan. Neutrinos passing through the detector scatter elastically from electrons in the water, and the recoiling electrons produce C̆erenkov light. This light is recorded by many inward facing photo-multiplier tubes (PMTs) that line the walls of the detector volume.

The original Kamiokande experiment consisted of $3 \mathrm{kT}$ of water, with 980 PMTs. The later Super-Kamiokande experiment was much larger, at $50 \mathrm{kT}$ with about 11,000 PMTs. The threshold of these detectors is set by their ability to detect the recoil electrons, which have an energy of about 5-7.5 MeV. Therefore, the Kamiokande experiments are looking at higher energy neutrinos than the other solar neutrino experiments, mainly neutrinos from reaction 3.3. As the energy and direction of the recoil electron can be measured, the original neutrino energy and direction can be inferred.

\subsubsection{SNO}

The Sudbury Neutrino Observatory (SNO) [33] is also a water C̆erenkov counter. It uses heavy water, $\mathrm{D}_{2} \mathrm{O}$, as apposed to standard $\mathrm{H}_{2} \mathrm{O}$. The benefit of this is that the SNO detector is able to distinguish between charged current (CC) events and neutral current (NC) events. Figure 3.2 shows the different interactions for CC and $\mathrm{NC}$ events in heavy water, as compared to those in normal water, which are shown in figure 3.3. In normal water counters, the two interactions are indistinguishable. However, in the heavy water of the SNO detector, the cross section of the neutrino interaction with the deuteron dominates.

The electrons produced from the CC events emit C̆erenkov radiation, which is detected by the PMTs. If the NC interaction energy transfer is above $2.2 \mathrm{MeV}$, the deuteron binding energy, a neutron is produced. Neutron capture then releases photons that can likewise be detected. 

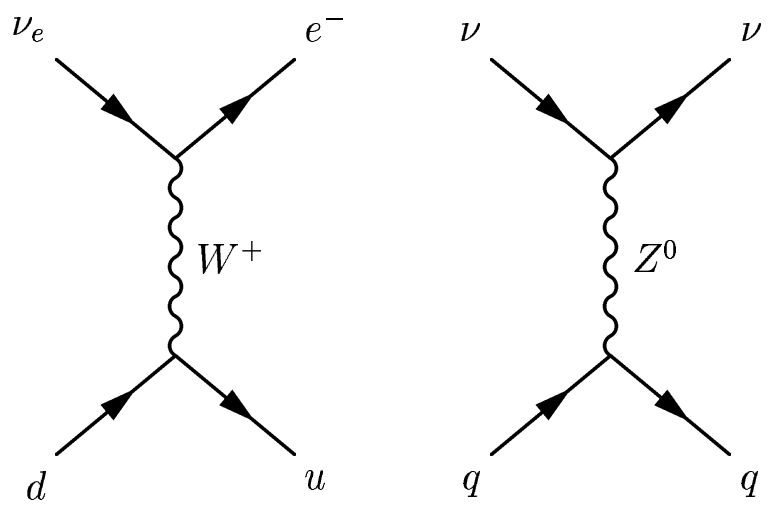

Figure 3.2: For deuteron interactions, the extra CC interaction available (left) can be distinguished from the NC interaction (right).
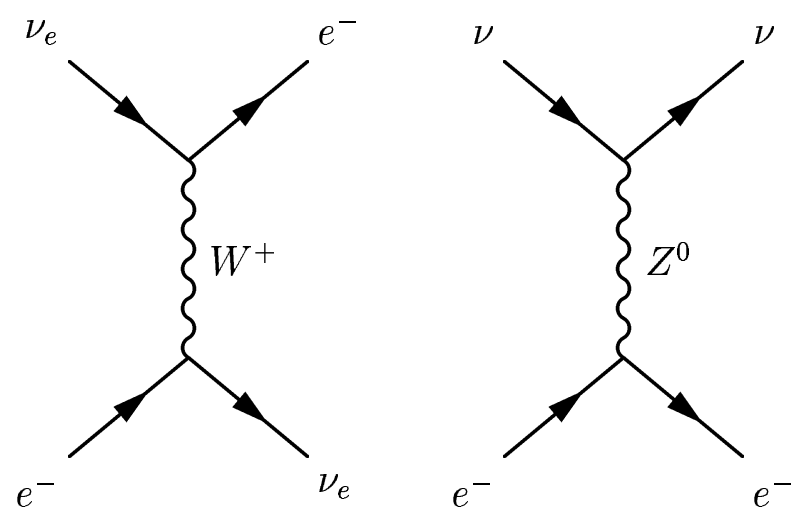

Figure 3.3: Neutrino interactions with light water. The charged current (left) and neutral current (right) are indistinguishable. 
The efficiency of this neutron capture in heavy water is low, at around $25 \%$. Because of this, SNO added sodium chloride to the water, bring the efficiency up to $\sim 85 \%$. A second method of detecting the neutron, using ${ }^{3} \mathrm{He}$ proportional counters, is also to be employed. It will provide a method of cross checking the NC interaction rate with different systematics providing a possibility of improving the precision.

\section{Total Rates: Standard Model vs. Experiment Bahcall-Pinsonneault 2000}

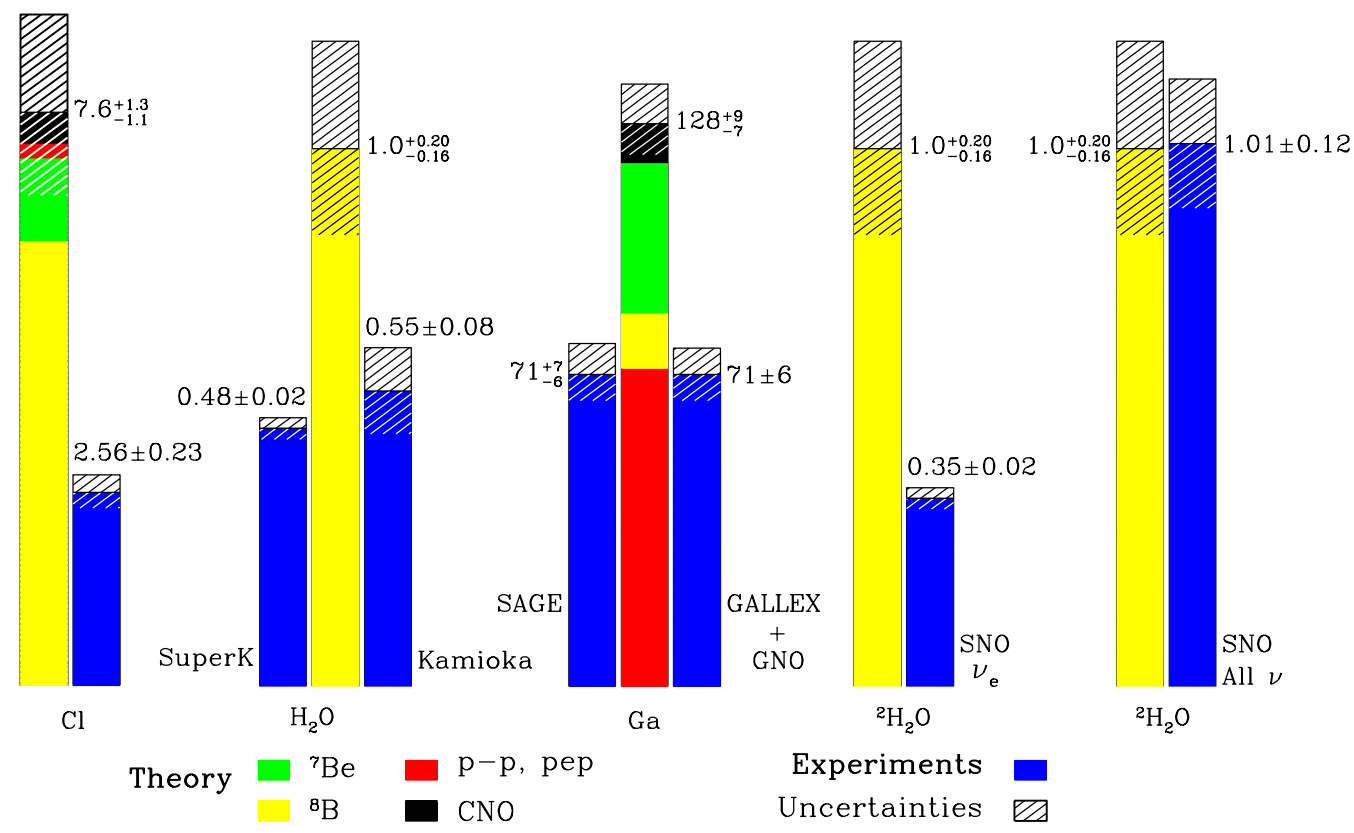

Figure 3.4: Summary of solar neutrino experimental results compared to theoretical expectations, from [24]. The first four sets of results show a deficit in the number of expected $\nu_{e}$ from Homestake, Super-Kamiokande, Kamiokande, GALLEX, GNO and SNO. The column on the right shows SNO's results for all neutrino flavours. They see a total number in agreement with predictions, implying that the neutrinos are all there, but that some have changed flavour. 
Figure 3.4 shows a summary of the solar neutrino experiment results. The diagram clearly shows a discrepancy between theoretical predictions and experimental results. The last two pairs of columns on the right show the most recent results from SNO $[34,35]$. The columns on the furthest right show that the total number of neutrinos detected agrees very well with theoretical predictions. However, the number of electron-neutrinos seen (set 4) is roughly a third of that expected. This is in agreement with the other solar-neutrino experimental results shown. This deficit strongly points towards a large number of $\nu_{e} \rightarrow \nu_{\tau}$ or $\nu_{e} \rightarrow \nu_{\mu}$ oscillations. SNO have announced a mass difference in a two-flavour model of $\Delta m^{2} \approx 5.0 \times 10^{-5}$ $\mathrm{eV}^{2}[35]$ from these results.

\subsection{Atmospheric Neutrinos}

Atmospheric neutrino experiments use cosmic-ray interactions in the upper atmosphere as their source of neutrinos. The cosmic rays interact with nucleons, producing an array of secondary particles - mainly kaons and pions. These mesons decay and provide part of the neutrino flux by producing $\nu_{\mu}$ and $\bar{\nu}_{\mu}$;

$$
\begin{aligned}
\pi^{ \pm} & \rightarrow \mu^{ \pm}+\nu_{\mu}\left(\bar{\nu}_{\mu}\right) \\
K^{ \pm} & \rightarrow \mu^{ \pm}+\nu_{\mu}\left(\bar{\nu}_{\mu}\right) .
\end{aligned}
$$

Further decays take place increasing the $\nu_{\mu}$ and $\bar{\nu}_{\mu}$ fluxes, and producing a $\nu_{\mathrm{e}}$ and $\bar{\nu}_{\mathrm{e}}$ flux,

$$
\mu^{ \pm} \rightarrow e^{ \pm}+\nu_{e}\left(\bar{\nu}_{e}\right)+\bar{\nu}_{\mu}\left(\nu_{\mu}\right)
$$

There is also a contribution to the neutrino fluxes from the decays

$$
\begin{aligned}
& K_{L} \rightarrow \pi^{+}+\ell^{-}+\bar{\nu}_{\ell} \\
& K^{+} \rightarrow \pi^{0}+\ell^{+}+\nu_{\ell}
\end{aligned}
$$


with $\ell=\mathrm{e}, \nu$. At low energies the most important process is the pion decay. At higher energies the kaon decays become more influential.

These interactions provide $\nu_{\mu}$ and $\nu_{e}$ in a ratio of approximately 2:1. Neutrino detectors are able to measure this ratio. It should be independent of the incident direction of the incoming neutrinos if there are no oscillations present. As the path lengths are different for neutrinos from different zenith angles, as shown in figure 3.5 , if there are oscillations present, the ratio will be distorted and will become a function of zenith angle.

In order for experiments to compare measurements of these ratios, it is common to quote results in terms of a ratio of ratios:

$$
\mathcal{R} \equiv \frac{\mathcal{R}_{\text {data }}}{\mathcal{R}_{\text {simulation }}}=\frac{\left(N_{\mu} / N_{e}\right)_{\text {data }}}{\left(N_{\mu} / N_{e}\right)_{\text {simulation }}}
$$

As the data were normalised by the simulation without oscillation for each experiment, $\mathcal{R}$ is in principle independent of the experiment involved. Therefore it is a good method by which to compare results from different experiments and technologies.

There have been two main types of atmospheric neutrino detectors to date: water C̆erenkov counters (IMB, Kamiokande and Super-Kamiokande), and fine-grained iron calorimeters (NUSEX, Frejus, Soudan 2). These experiments are able to monitor atmospheric neutrino fluxes with energies in the range from $1 \mathrm{GeV}$ to several hundred GeV.

The atmospheric neutrino deficit was first measured by the IMB experiment, a water Čerenkov detector situated in the Morton mine in the USA. The IMB collaboration announced a value for $\mathcal{R}$ of $0.54 \pm 0.05[36,37]$. These results were confirmed two years later by Kamiokande [38].

A few years later, NUSEX [39,40] and Frejus [41] announced a result for $\mathcal{R}$ of very close to unity. However, Soudan 2 [42] was also a fine grained tracking 
iron calorimeter and their results, which had higher statistics that the previous iron detectors, agreed with the ratio seen by Kamiokande and IMB.

\subsubsection{Super-Kamiokande}

The Super-Kamiokande experiment has provided us with the best measurement yet of the atmospheric neutrino deficit. As figure 3.5 shows, by recording the direction of the incoming neutrinos, the distance that they must have travelled since their creation can be calculated. This gives a much stronger base for the calculation of the neutrino oscillation parameters. Using this extra information, Super-Kamiokande announced a value for $\mathcal{R}$ of about 0.6 in 1998 [43].

Most importantly, they also reported a variation in the $\nu_{\mu}$ flux with the zenith angle in a manner that would be consistent with neutrino oscillations. This is shown in figure 3.7. The larger the zenith angle, i.e. the smaller $\cos \theta$, the greater the distance the neutrino had travelled. The $\nu_{e}$ spectrum was however recorded as expected. This led to the conclusion that the oscillations $\nu_{\mu} \rightarrow \nu_{\tau}$ or $\nu_{\mu} \rightarrow \nu_{\text {sterile }}$ must be the culprit.

More data, and further analysis [44], have enabled the exclusion of $\nu_{\mu} \rightarrow \nu_{\text {sterile }}$ to a $99 \%$ C.L. The value of $\Delta m^{2}$ has also been calculated to be $\approx 2.5 \times 10^{-3} \mathrm{eV}^{2}$ [45] assuming a two flavour mixing.

\subsection{Reactor Neutrinos}

During nuclear fission $\bar{\nu}_{e}$ 's are created as a byproduct. These neutrinos have an energy of $\sim 3 \mathrm{MeV}$. To detect these neutrinos, experiments are placed at distances ranging from $10 \mathrm{~m}$ to $1 \mathrm{~km}$ from the source. The $\bar{\nu}_{e}$ are detected via the inverse beta decay interaction

$$
\bar{\nu}_{e}+p \rightarrow \mathrm{e}^{+}+n
$$

Detectors operating at these distances from a neutrino source provide a method 


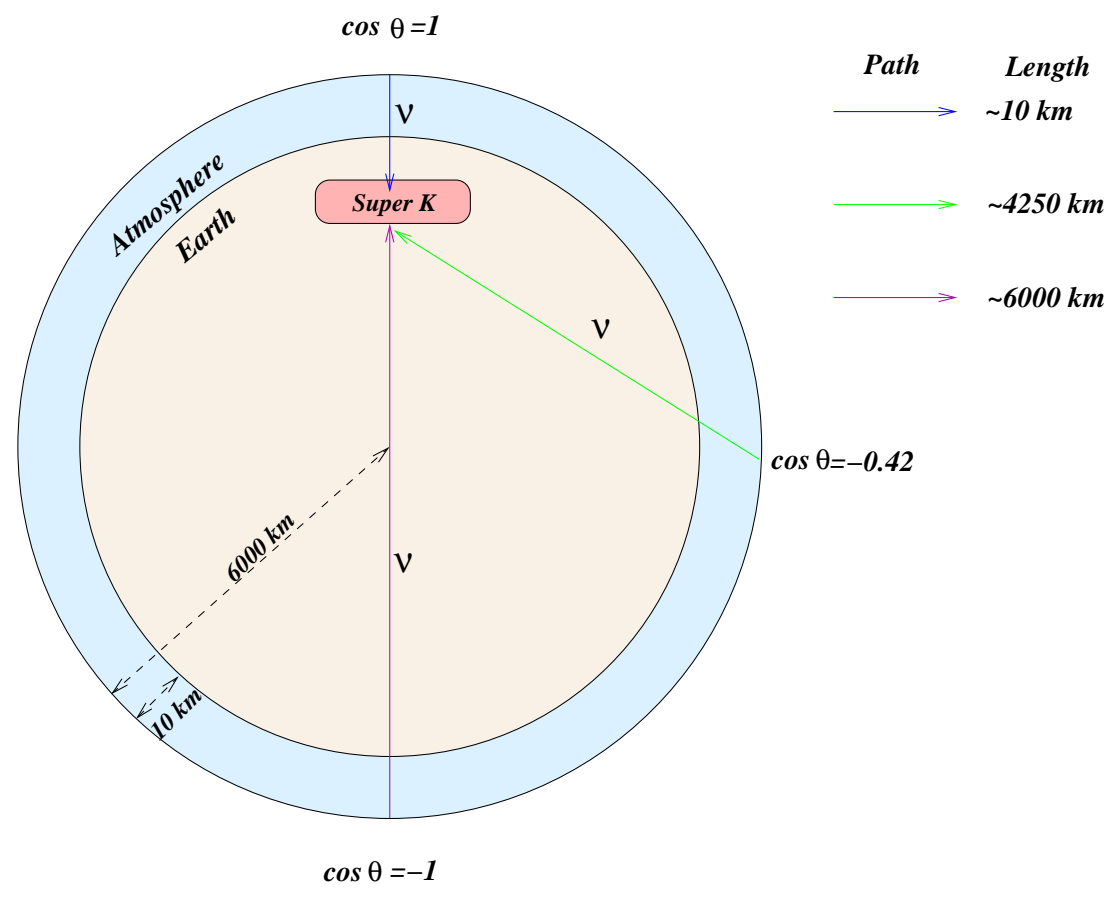

Figure 3.5: Due to its ability to identify the direction of the incoming neutrino, Super-Kamiokande is able to classify neutrino interactions by zenith angle (Not to scale). 


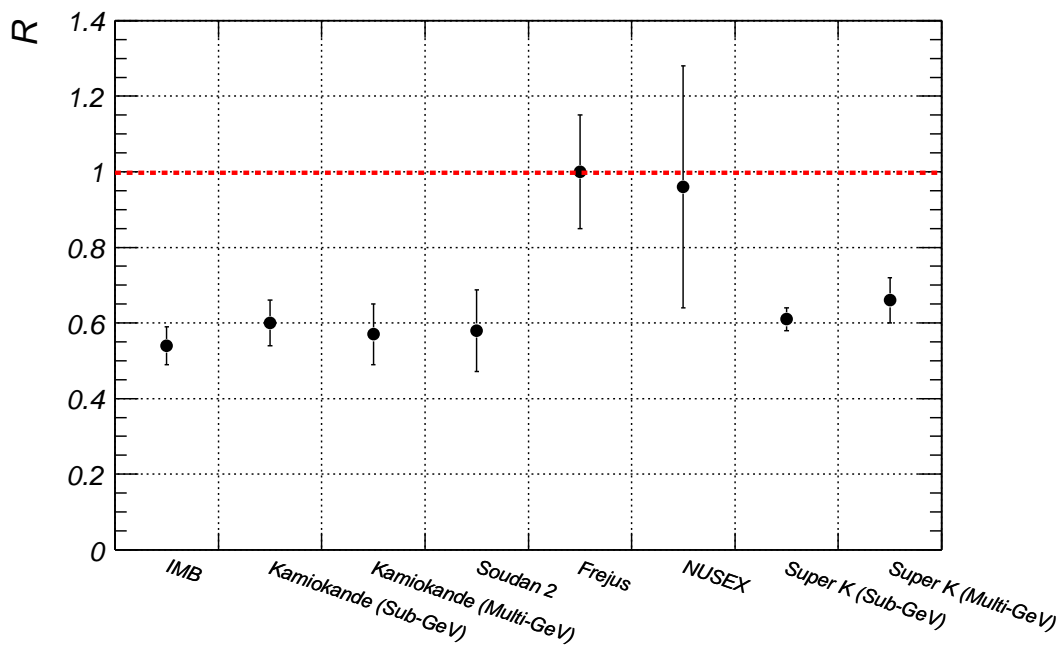

Figure 3.6: The ratio of ratios measured by the major atmospheric neutrino experiments. The red line represents the situation with no neutrino oscillations.

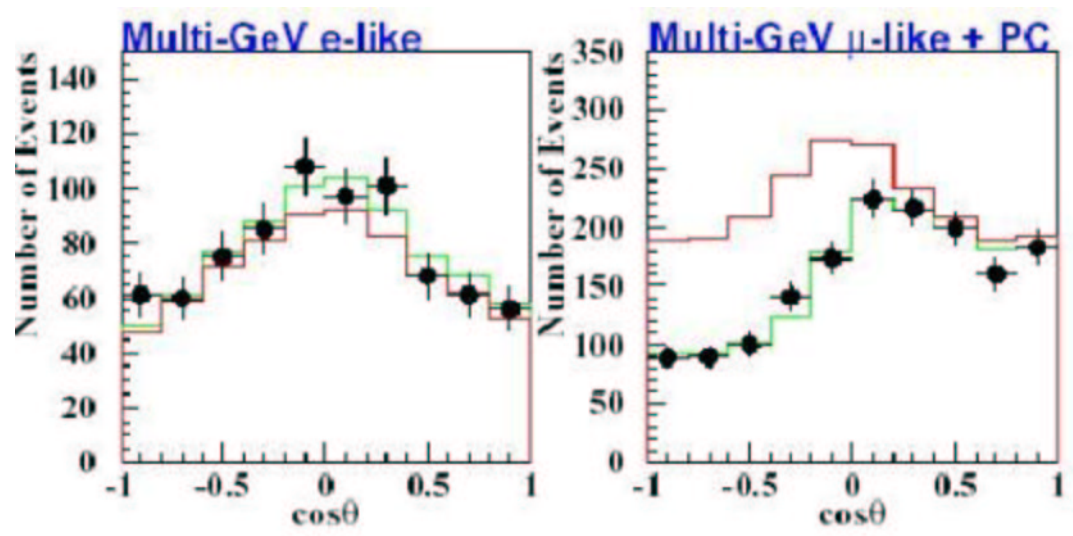

Figure 3.7: These results from Super-Kamiokande show strong evidence for neutrino oscillations. The left-hand plot shows that the number of $\nu_{e}$-like events observed did not vary with distance from the point of creation. The right-hand plot clearly shows a deficit in $\nu_{\mu}$ like events with increasing zenith angle. This is consistent with $\nu_{\mu} \rightarrow \nu_{\tau}$ oscillations. 
of detecting $\Delta m^{2}$ in the region of $\sim 10^{2} \mathrm{eV}^{2}$. Until recently, the most sensitive reactor experiment was the CHOOZ [46] detector. The experiment lasted until 1998. When all of the data from their running was analysed no discrepancy in the $\bar{\nu}_{e}$ flux was seen. This allowed a large part of the $\nu_{e}, \nu_{\mu}$ neutrino oscillation phase space to be ruled out. They also confirmed the results from the Super-Kamiokande experiment, suggesting that $\nu_{\mu} \rightarrow \nu_{e}$ oscillations were not the cause of the deficit.

\subsubsection{KamLAND}

The Kamioka Liquid-scintillator Anti-Neutrino Detector experiment (KamLAND) is a 1000 ton liquid scintillator detector based in the old Kamiokande cavern. KamLAND searched for the oscillation of $\bar{\nu}_{e} \mathrm{~s}$ emitted from distant nuclear power reactors. The baseline had a typical length of $180 \mathrm{~km}$, and the anti-neutrinos were detected via inverse beta decay shown above, with energies over 1.8 MeV. They have recently announced results for reactor anti-neutrino disappearance [47]. Figure 3.8 show the predicted and actual neutrino spectra seen.

Figure 3.9 shows their results for $162 \mathrm{kTy}$ (kiloton-years) of data. They produced a best-fit solution of $\sin ^{2} 2 \theta=1.0$ and $\Delta m^{2}=6.9 \times 10^{-5} \mathrm{eV}^{2}$.

\subsection{Accelerator Experiments}

Accelerator experiments are the only neutrino oscillation search experiments that produce their own controlled source of neutrinos. They are therefore able to select the energy of the neutrinos that they fire at the detectors. Accelerator experiments fall into two categories: short baseline, where the detector is typically less than 1 $\mathrm{km}$ from the source; and long baseline, where the detector is based hundreds of kilometres from the source. 


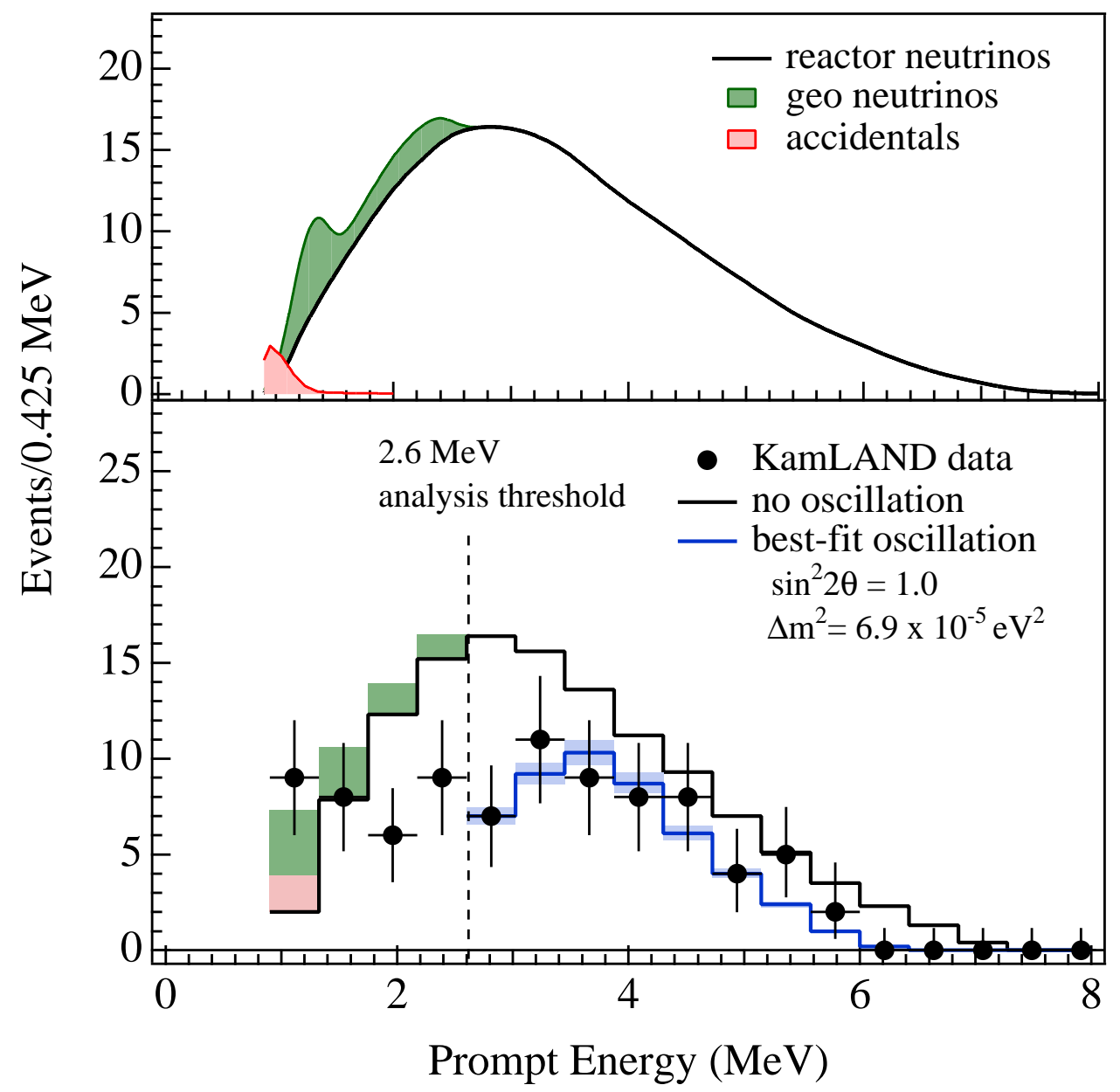

Figure 3.8: Upper panel: The reactor $\bar{\nu}_{e}$ energy spectrum expected. Lower panel: Energy spectrum of the observed events (solid circles with error bars), along with the expected no oscillation spectrum (upper histogram) and best fit (lower blue histogram) including neutrino oscillations. The shaded band indicates the systematic error in the best-fit spectrum. The vertical dashed line corresponds to the analysis threshold at 2.6 MeV, from [47]. 


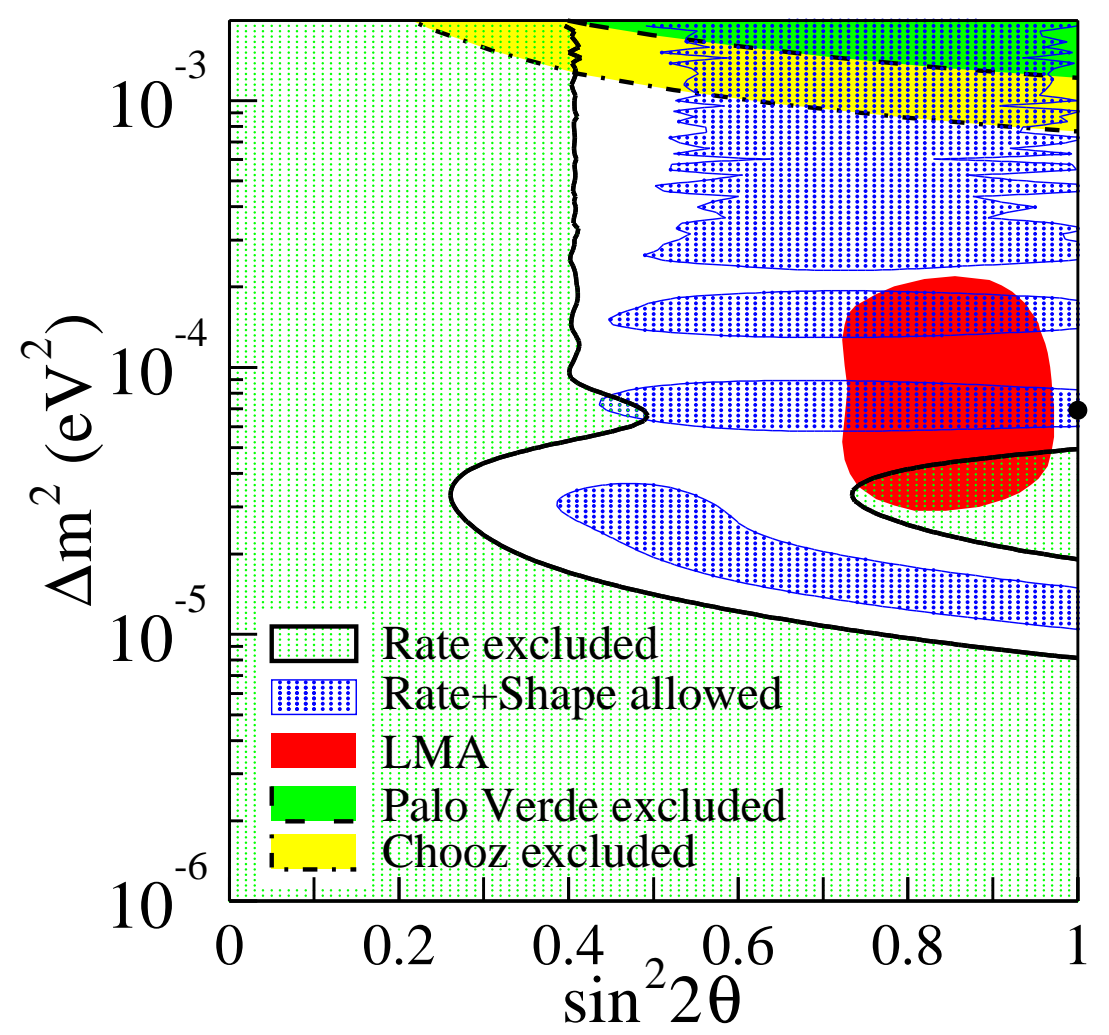

Figure 3.9: Excluded regions of $\nu_{e}$ and $\nu_{\mu}$ neutrino oscillation parameters from KamLAND at 95\%C.L. The thick dot represents the KamLAND best fit in the region: $\sin ^{2} 2 \theta=1.0$ and $\Delta m^{2}=6.9 \times 10^{-5} \mathrm{eV}^{2}$, from [47]. 


\subsubsection{Short-Baseline Experiments}

Accelerators used in short-baseline experiments produce neutrinos with energies ranging from $10 \mathrm{MeV} \rightarrow 10 \mathrm{GeV}$. This allows them to analyse the oscillation phase space down to $\Delta m^{2} \sim 0.1 \mathrm{eV}^{2}$.

CHORUS [48] and NOMAD [49] were two short-baseline experiments based at CERN. They were both looking for $\nu_{\tau}$ appearance through $\nu_{\mu} \rightarrow \nu_{\tau}$ oscillations. No signal was found, and therefore a portion of $\nu_{\mu} \rightarrow \nu_{\tau}$ phase space has been excluded for $\Delta m^{2}>1 \mathrm{eV}^{2}$.

The only accelerator-based experiment so far to have shown any positive evidence of neutrino oscillations is the Liquid Scintillator Neutrino Detector (LSND) [50] at the Los Almos Meson Physics Facility.

The LSND experiment used neutrinos produced from two different mechanisms. A proton beam was fired into a water target to create $\pi^{ \pm}$. These decay via the following chain:

$$
\begin{aligned}
& \pi^{ \pm} \rightarrow \mu^{ \pm}+\nu_{\mu}\left(\bar{\nu}_{\mu}\right) \\
& \mu^{ \pm} \rightarrow e^{ \pm}+\nu_{e}\left(\bar{\nu}_{e}\right)+\bar{\nu}_{\mu}\left(\nu_{\mu}\right) .
\end{aligned}
$$

There are two forms of analysis carried out on the detected neutrinos. For the decay-at-rest (DAR) analysis, the $\pi^{+}$would stop and decay in a water-cooled copper beam dump. The resulting muons would decay as above, providing a source of $\bar{\nu}_{\mu} \mathrm{s}$. The oscillation $\bar{\nu}_{\mu} \rightarrow \bar{\nu}_{e}$ was then sought, with $\bar{\nu}_{e}$ being detected via the annihilation of the positron from $\bar{\nu}_{e}+p \rightarrow e^{+}+n$. A delayed 2.2 MeV $\gamma$ is also detected from the neutron capture. The decay-in-flight (DIF) analysis used the $\nu_{\mu}$ from the $\pi^{-}$ decay to look for $\nu_{\mu} \rightarrow \nu_{e}$ oscillations. $\nu_{e}$ were to be detected via $\nu_{e}+\mathrm{C} \rightarrow \mathrm{e}^{-}+\mathrm{N}$ in the organic scintillator.

A comparison of these events allowing for background showed an excess of neutrinos. This excess allowed a further region of phase space to be denoted as 
allowed; this corresponded to $\Delta m^{2} \sim 1 \mathrm{eV}^{2}$ and $\sin ^{2} 2 \theta \sim 10^{-2}$.

\subsubsection{Long-Baseline Experiments}

The first long-baseline to start taking data was the K2K, (KEK to Super-Kamiokande), experiment. This has a baseline of 250 miles, and uses a beam of $\nu_{\mu}$ with an energy of around the $1.3 \mathrm{GeV}$. Initial results from $\mathrm{K} 2 \mathrm{~K}$ seem to indicate $\nu_{\mu} \rightarrow \nu_{\tau}$ oscillations in the region suggested by Super-Kamiokande for atmospheric neutrinos. However, there has been no formal interpretation so far [51].

Another long-baseline experiment in the pipeline is NCGS, (Neutrinos from CERN to Gran Sasso). Again using a beam of $\nu_{\mu}$, this time fired from CERN to Gran Sasso, two existing detectors are to be used: The OPERA emulsion based detector, and the ICARUS liquid-argon time projection chamber. Both will be searching for $\nu_{\tau}$ appearance.

MINOS (Main Injector Neutrino Oscillation Search) is another long-baseline experiment, under construction in the USA. MINOS will allow the first precision measurements of the neutrino mixing parameters. It is explained in detail later in this thesis.

\subsection{Summary of Results}

The standard method of displaying neutrino oscillation results is in the form of parameter-space diagrams for $\sin ^{2} 2 \theta$ and $\Delta m^{2}$. Figure 3.10 shows the allowed and excluded regions for $\nu_{\mu} \rightarrow \nu_{\tau}$ oscillations from various experiments. Figure 3.11 shows the regions selected for the more popular $\nu_{\mu} \rightarrow \nu_{e}$ oscillation searches. 


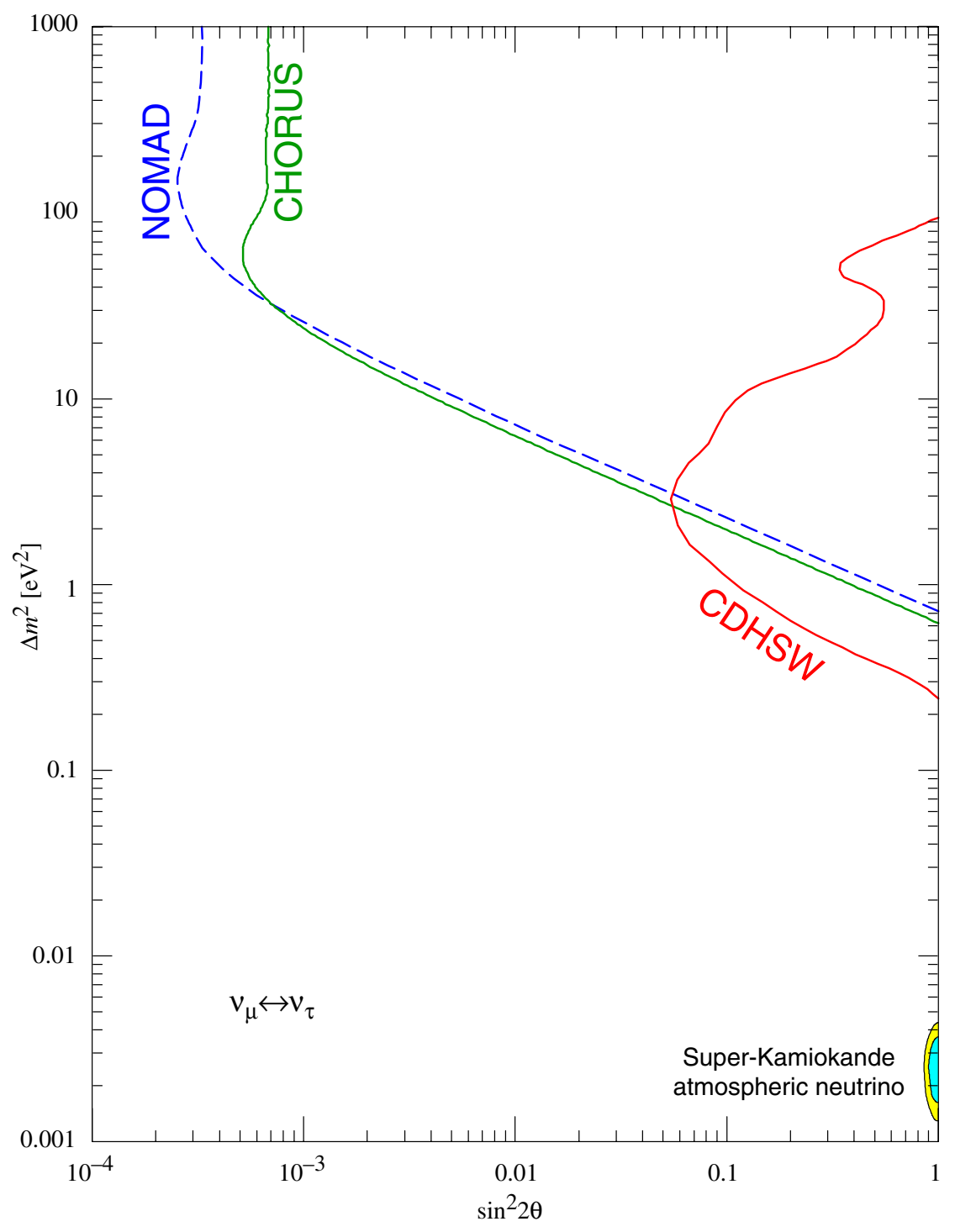

Figure 3.10: Shown here are the allowed and excluded regions of the neutrinooscillation parameter space for $\nu_{\mu} \rightarrow \nu_{\tau}$. The excluded regions are from the short baseline experiments Nomad, Chorus and CDHSW at CERN. The allowed parameter space from Super-Kamiokande is also shown. [52] 


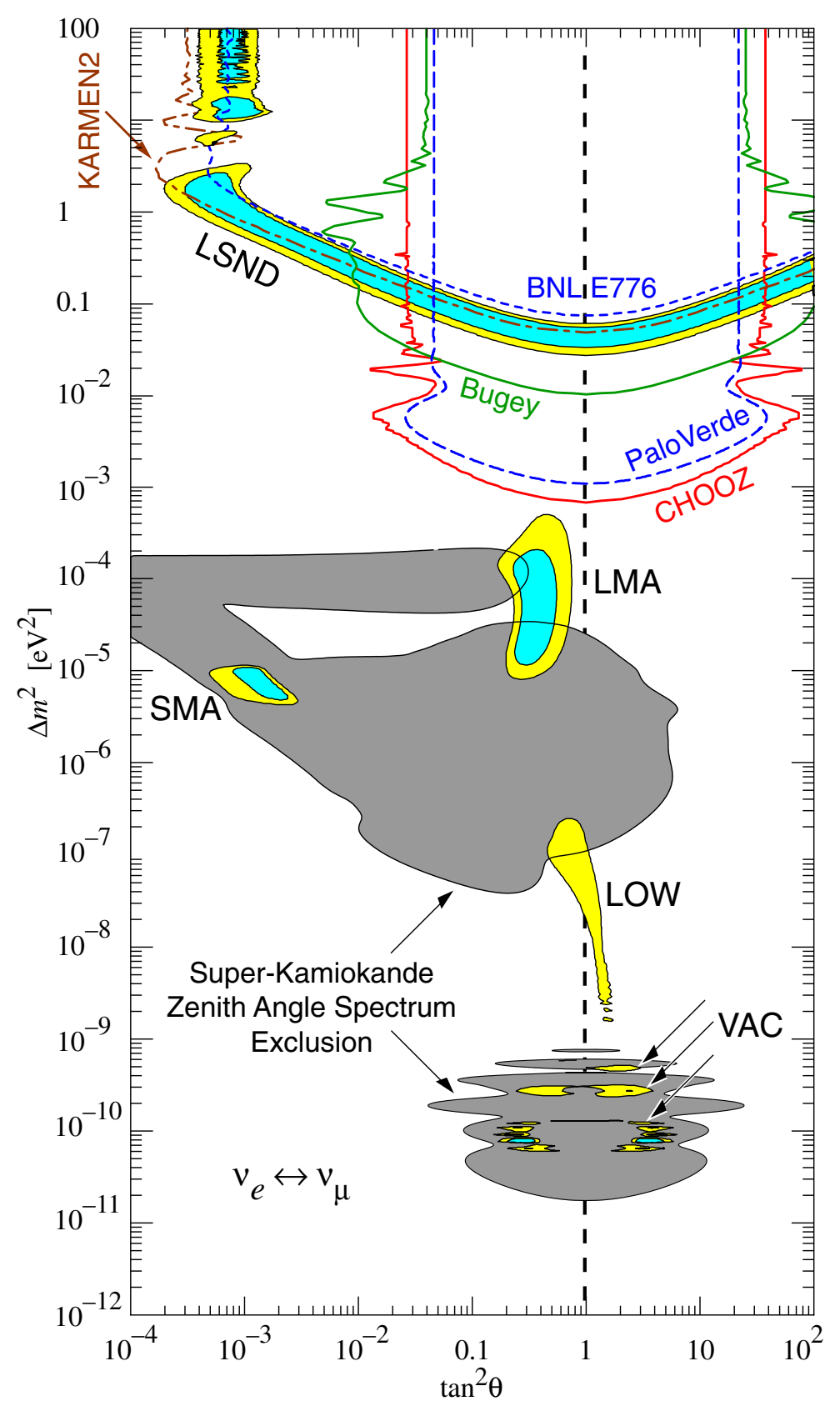

Figure 3.11: Shown here are the allowed and excluded regions of neutrino oscillation parameter space for $\nu_{\mu} \rightarrow \nu_{e}$. The allowed region from LSND is shown, along with the excluded regions from the short baseline experiments, Karmen2, Bugey and BNL E776. Also shown are the excluded regions from the reactor experiments $\mathrm{CHOOZ}$ and Palo Verde. The solar neutrino experiment results from SAGE, GALLEX, GNO, Super-Kamiokande and SNO are shown as Large Mixing Angle (LMA), Small Mixing Angle (SMA), Low $\Delta m^{2}$ (LOW) and VAC (vacuum “just so" solutions). [52] 


\section{Chapter 4}

\section{MINOS Overview}

The Main Injector Neutrino Oscillation Search (MINOS) is a long-baseline neutrino oscillation experiment. As previously discussed, to detect neutrinos you need detectors with a very large mass, to increase the probability of a neutrino decaying within them. You also need to be able to measure the neutrino spectra at two points. This allows the spectra of the neutrino beam to be compared, and the appearence or disappearence of any detectable flavour to be measured.

To achieve this the MINOS experiment employs two main detectors, separated by $735 \mathrm{~km}$, known as the "near" and "far" detectors. Both these detectors contain a large amount of iron to increase their mass, and hence their ability to detect neutrinos. The near detector is based only $1 \mathrm{~km}$ from the beam target, and is expected to receive 130,000 events per day. At the far detector only 4 events per day are expected, and so reduction of background noise is very important. This is achieved in part by situating the detector inside a former iron mine, where it is covered by 2341 feet of rock.

The beam is to be generated by the NuMI beam-line under construction at Fermilab, near Chicago. Protons produced at the Main Injector at Fermilab will be 
used to generate a neutrino beam consisting mainly of $\nu_{\mu}$. This beam will be directed northwest through the Earth to the Soudan mine in northern Minnesota, 735 $\mathrm{km}$ away, as shown in figures 4.1 and 4.2.

Full details of the detectors are available in the Technical Design Reports [53, 54]; however, I will outline the detector construction and beam details here.

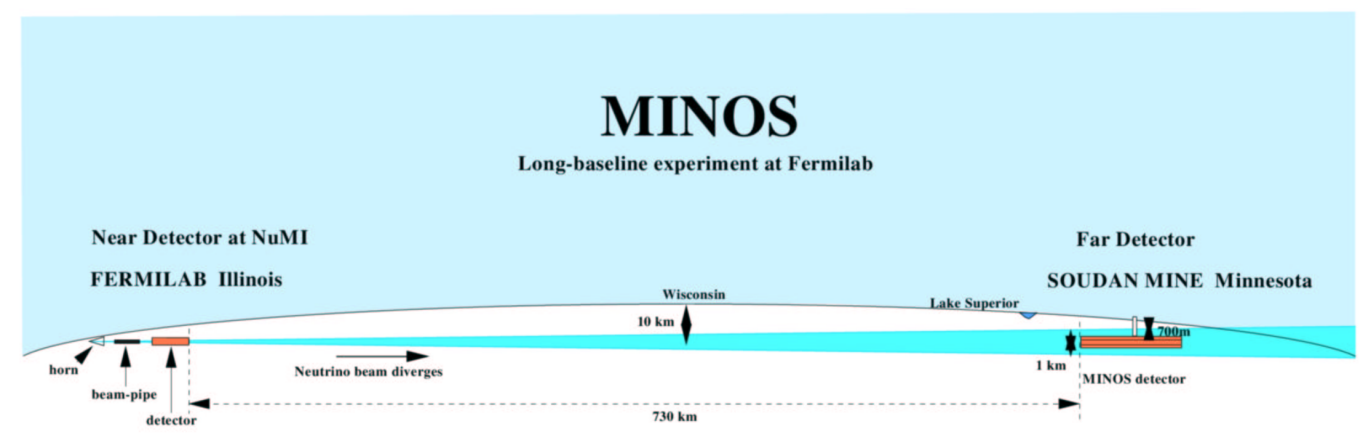

Figure 4.1: The route taken by the neutrino beam through the Earth

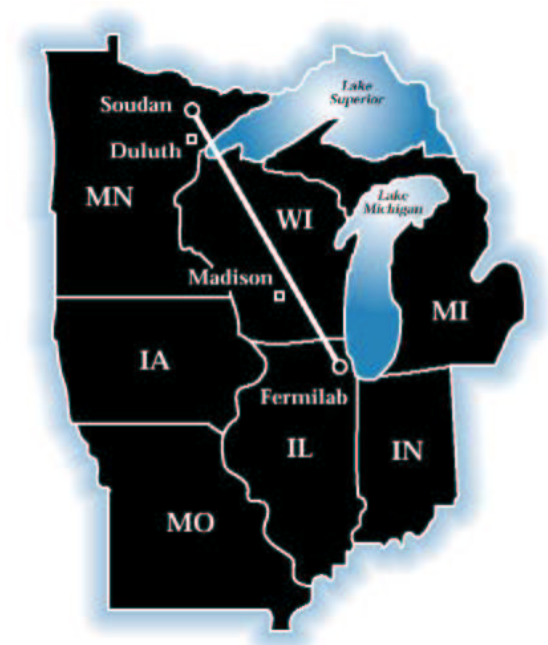

Figure 4.2: The route taken by the neutrino beam overlaid on a map of the Great Lakes region of the USA. 


\subsection{NuMI Beam}

The Minos experiment will use the new NuMI (Neutrinos at the Main Injector) [55] neutrino beam line which has been constructed at Fermilab.

The Main Injector at Fermilab focuses $120 \mathrm{GeV}$ protons on to a $1 \mathrm{~m}$ long carbon target. This generates secondary mesons, mostly $\mathrm{K}$ and $\pi$. These are directed by two magnetic focusing horns, which select positive pions, down a $675 \mathrm{~m}$ decay pipe. In the decay pipe, the pions decay via $\pi^{+} \rightarrow \mu^{+}+\nu_{\mu}$ to generate the neutrino beam. A hadron absorber then removes any hadrons that have not decayed. There is then $290 \mathrm{~m}$ of rock between the hadron absorber and the near detector. This will give most of the muons enough distance to range out. The result is a beam of mainly $\nu_{\mu}$ with a small contamination of $\nu_{e}$. Figure 4.3 shows a schematic of this production method.

There are three choices of energy available for the NuMI beam: low, medium and high. Figure 4.4 shows the different energy spectra and configurations of the focusing horns. The MINOS collaboration has recently decided to run with the low energy beam, with the option of changing to a higher energy in the future. This will initially allow the parameter space suggested by recent experiments such as Super-Kamiokande to be investigated.

The NuMI beam will be the most intense neutrino source to date. The Main Injector will provide $4 \times 10^{13}$ protons per spill. A spill occurs every 1.9 seconds and is $8.1 \mu \mathrm{s}$ long. Even with a beam this intense, MINOS is only expected to observe $300 \mathrm{CC}$ events/kT/year at the far detector, assuming no oscillations.

For the MINOS experiment to make the most sensitive measurements it is vital that the beam generation dynamics are very well understood, so that the neutrino spectrum is known exactly. To do this an experiment has been designed to measure the properties of $\mathrm{p}-\mathrm{C}$ collisions. Known as MIPP, Main Injector Particle Production, it is under construction and will start taking data in the next couple of years. The 


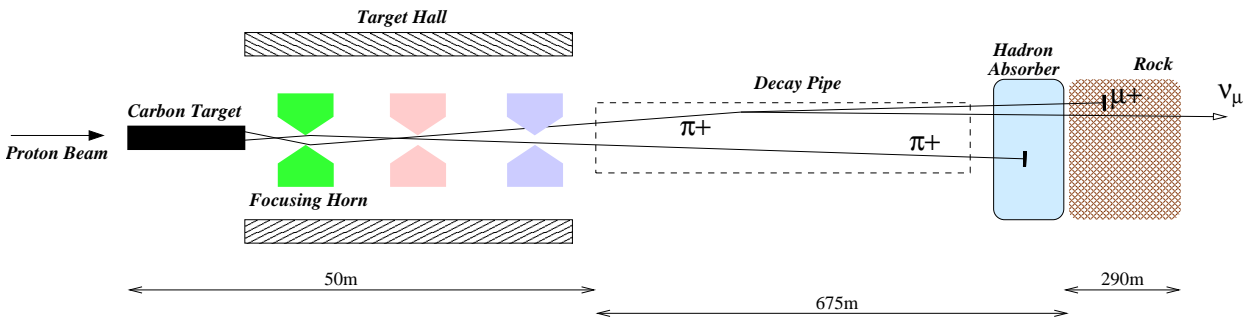

Figure 4.3: Schematic of the NuMI beam line. This schematic shows how the neutrinos to be used in the MINOS experiment are produced. The proton beam from the accelerator can be seen hitting the target, where the resulting particles, mainly pions and kaons are produced. The $\pi^{+}$are then focused into a beam by the focusing horn. The heavily shaded green horn shows the low energy configuration. The lighter shaded red and blue horns show the medium and high energy alternative setups.
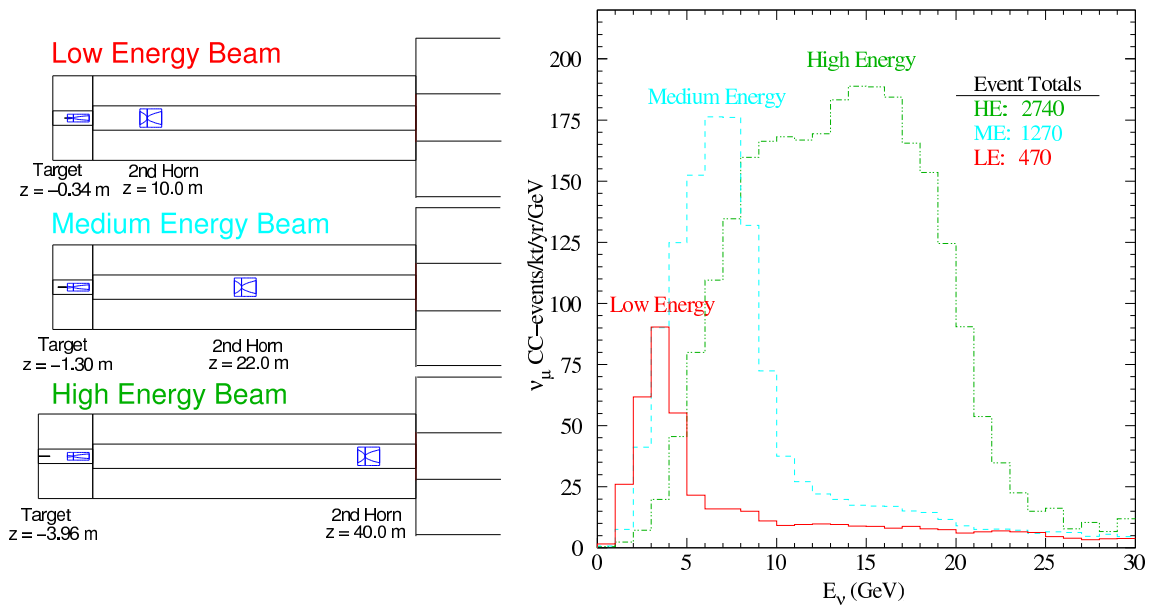

Figure 4.4: Energy spectra for the three different configurations of the NuMI beam generation equipment. MINOS has decided to opt for the low energy configuration, producing $\approx 2400 \nu_{\mu} \mathrm{CC}$ events per year at the far detector. 


\begin{tabular}{|c|c|}
\hline Proton energy & $120 \mathrm{GeV}$ \\
\hline Time Structure & $8.1 \mu$ s every 1.9 seconds \\
\hline Intensity & $3.8 \times 10^{13}$ protons/spill \\
\hline Beam Generation & $1 \mathrm{~m}$ graphite target, 2 horn pion focusing \\
\hline$\nu$ Beam Spectrum & Low Energy 1-6 GeV $\nu_{\mu}$ with high tail to $50 \mathrm{GeV}$ \\
\hline Baseline & $735.340 \mathrm{~km}$ \\
\hline Detector Type & $2.54 \mathrm{~cm}$ iron, $1 \mathrm{~cm}$ scintillator. \\
\hline Detector Mass & Far - 5.4 kT, Near - $680 \mathrm{~T}$, Calibration - $1 \mathrm{~T}$ \\
\hline B field & $1.5 \mathrm{~T}$ average \\
\hline \multirow[t]{2}{*}{ Resolution } & Hadronic $60 \% / \sqrt{(} E)$ \\
\hline & Electromagnetic $25 \% / \sqrt{(} E)$ \\
\hline \multirow[t]{2}{*}{ Event Rate } & Near detector - 3 events/spill \\
\hline & Far Detector - $300 \nu_{\mu} \mathrm{CC}$ events/kT/yr (no osc) \\
\hline
\end{tabular}

Table 4.1: Summary of the MINOS parameters 
data from this experiment will help to refine the Monte Carlo simulations used in the flux calculations.

As a preliminary measurement, a group, of which I was a member, carried out a number of runs on the NA49 detector at CERN to investigate $\mathrm{p}-\mathrm{C}$ collisions. This measurement is presented in detail later in this thesis, in chapter 7. These measurements will provide valuable points of reference for the MIPP experiment.

\subsection{Detectors}

In addition to the near and far detectors, there is a so called calibration detector designed to measure the MINOS detector response in different test beams at CERN. This allows calibration to be made of the other two detectors. To reduce systematic errors, all three detectors are built with very similar designs.

The detectors are all coarse-grained tracking calorimeters. They are designed with a steel-scintillator-air sandwich structure with the same $6 \mathrm{~cm}$ pitch between planes. Each "plane" of the detector consists of a 1" thick sheet of steel backed with plastic scintillator strips. The scintillators are encased in an aluminium module to provide light tightness. There is also a small air gap between each pair of planes to allow for local variations in the flatness of the steel.

The scintillator strips are extruded from $663 \mathrm{~W}$ polystyrene from the DOW chemical company. The scintillator strips are doped with impurities of fluoring PPO at a $1 \%$ level, and a wavelength shifter (POPOP) at $0.030 \%$. They are $1 \mathrm{~cm}$ thick and $4.1 \mathrm{~cm}$ wide, and they run the width of the detector. A $0.25 \mathrm{~mm}$ layer of $\mathrm{TiO}_{2}$ is coextruded with the polystryene to maximize internal reflection along the strips. Each strip has a groove cut into it, along which lies a length of $1.2 \mathrm{~mm}$ Kurary Y11 wavelength shifting fibre (WLS) running the whole length of the strip. These fibres are optically coupled to the scinitilator strips with epoxy, and provide the signal output of each strip. 
Both the scintillator and the readout fibres have wavelength shifting properties to increase the light yield aquired by the detector. By shifting the wavelength of the light to one in tune with the fibre you increase the probability of the light being captured in the fibre. Hence you also increase the ability of the detector to detect low energy interactions which produce very little light.

The WLS fibres are coupled to cables of clear signal fibres at the end of each module. These readout cables lead to light tight boxes containing the PMTs that provide the optical readout, known as MUX boxes. Adjacent planes of the detector are rotated by $90^{\circ}$, to provide three-dimensional read out of the detector.

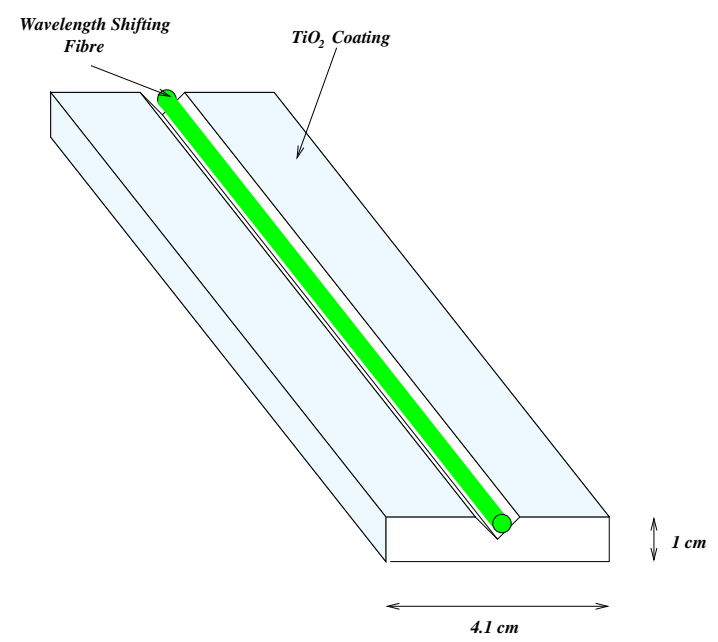

Figure 4.5: Schematic of the scintillator strip construction, showing the position of the wavelength-shifting signal fibre and the reflective coating.

Both the near and far detectors are magnetised by current-carrying coils. The coils provide a magnetic field ranging from 1 to $1.5 \mathrm{~T}$. This magnetic field provides a means to measure the charge and momentum of muons by their track curvature. The calibration detector is not magnetised, studies done investigating the influence of a magnetic field [56] have shown that a magnetic field does not affect either the energy resolution of the detector, or the mean of the energy distribution. Due to this, and to the problems that would be encountered in creating a magnetic field 
of strength comparable to the far or near detector, the calibration detector is not magnetised.

The detectors have a resolution $\frac{\sigma_{(E)}}{E}$ of $\sim 23 \% / \sqrt{E}$ for electromagnetic showers, and $\sim 55 \% / \sqrt{E}$ for hadronic showers, where $\mathrm{E}$ is measured in GeV. Multiple Coulomb scattering in the steel sections of the planes limits the resolution, such that a $10 \mathrm{GeV}$ muon can have its momentum measured to $\pm 14 \%$ [57]. For stopping muons the momentum can be measured far more accurately, to $\pm 6 \%$, by considering its range [57].

\subsubsection{Far Detector}

The far detector is the biggest of all three MINOS detectors, with a mass of 5.4 kT. There are 486 planes, each each of which is an $8 \mathrm{~m}$ octagon of steel, backed with aluminium scintillator modules. Access to the mine is only possible via a $2 \mathrm{~m}$ square shaft, requiring everything to be split into sections and reconstructed down the mine. Therefore each steel plane is constructed from 4 subsections each only 2 m wide. Each scintillator plane has 192 scintillator strips, and is constructed from 8 aluminium modules: four modules with 28 strips, and four with 20 strips. The detector is split into 2 so called "super-modules". Each super-module is individually magnetised via a $15 \mathrm{kA}$-turn coil. This provides a magnetic field of $1.5 \mathrm{~T}, 2 \mathrm{~m}$ from the centre. The field produced by this coil is shown in figure 4.7.

Every plane is read out on all 192 strips at both ends. The readout cables are bundled together, so each bundle contains all the fibres from one side of two planes. This bundle of fibres then leads to a Multiplexing (MUX) box. In this box the individual fibres from each strip are bundled into groups of eight, with each bundle leading to one pixel from three 16 pixel PMTs. Each PMT then reads a total of 128 channels, 8 fibres on each of its 16 pixels.

The fibres are grouped in such a way that none of the 8 strips read out by a bundle is less than $1 \mathrm{~m}$ from its nearest neighbour, as shown in figure 4.8. This 


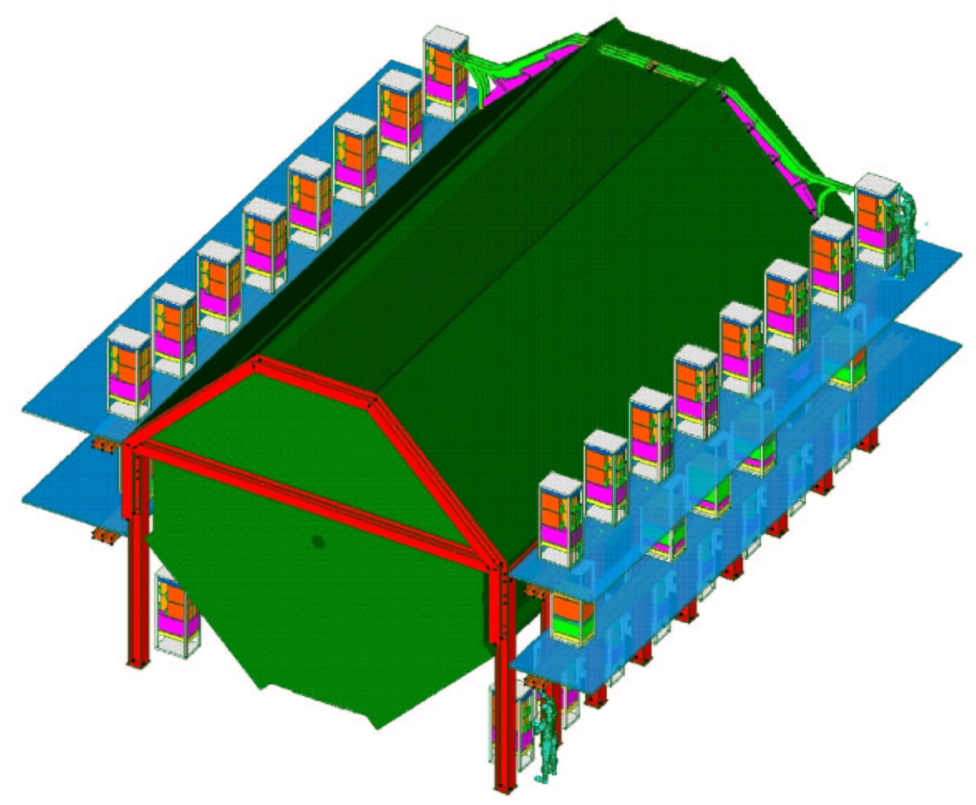

Figure 4.6: Schematic of the far detector. It shows one supermodule, i.e. half of the full detector, and the cable detail for one plane, towards the rear. The instrumentation crates can also be seen. In this picture the coil is missing; however the hole for the coil can just be made out in the centre of the detector.

multiplexing is possible due to the low event rate at the far detector. As a typical event will have a width of around $50 \mathrm{~cm}$, it is unlikely to deposit light in the same pixel twice from different fibres. As the multiplexing pattern is different on each side of the detector, unambiguous event reconstruction can in principle be done by software de-multiplexing of the data. The result of this multiplexing of signal fibres is that only 1452 PMTs are required to read out the entire detector. There is another complication to the multiplexing involving the light-injection calibration system; this is explained later in chapter 6 .

Digitisation of the PMT signals is done by customised Viking VA Chips [58], constructed by IDE AS of Norway [59]. Each Viking chip has 32 channels of amplification, shaping and holding circuitry, allowing each channel to be read out in turn. Each MUX box has a front end board containing 3 sampling chips, one for 

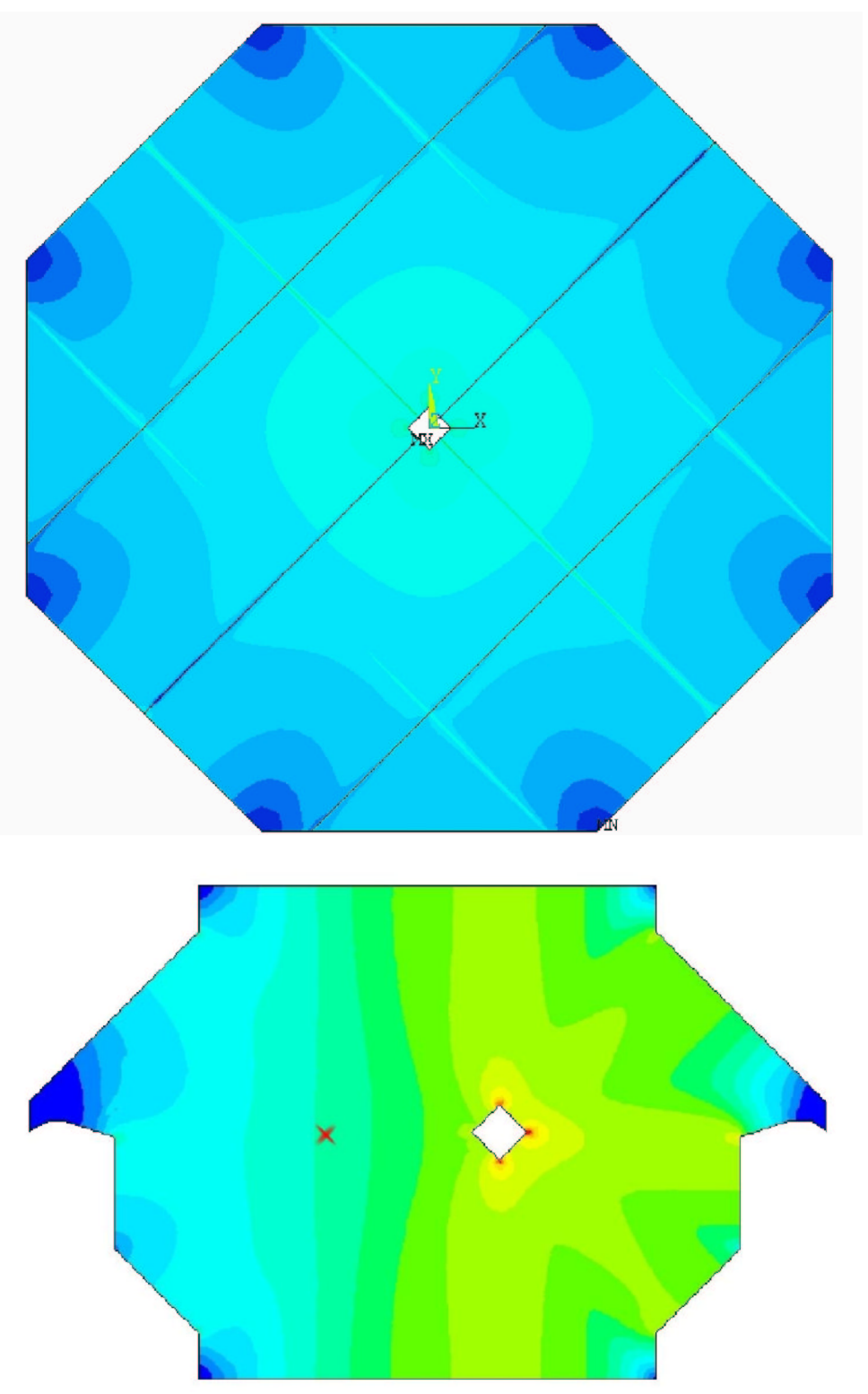

Figure 4.7: Comparison of models of the magnetic fields of the near (bottom) and far (top) detectors. The diamond holes show the coil locations, and the red cross on the near detector map shows the beam target point (Not to scale). 

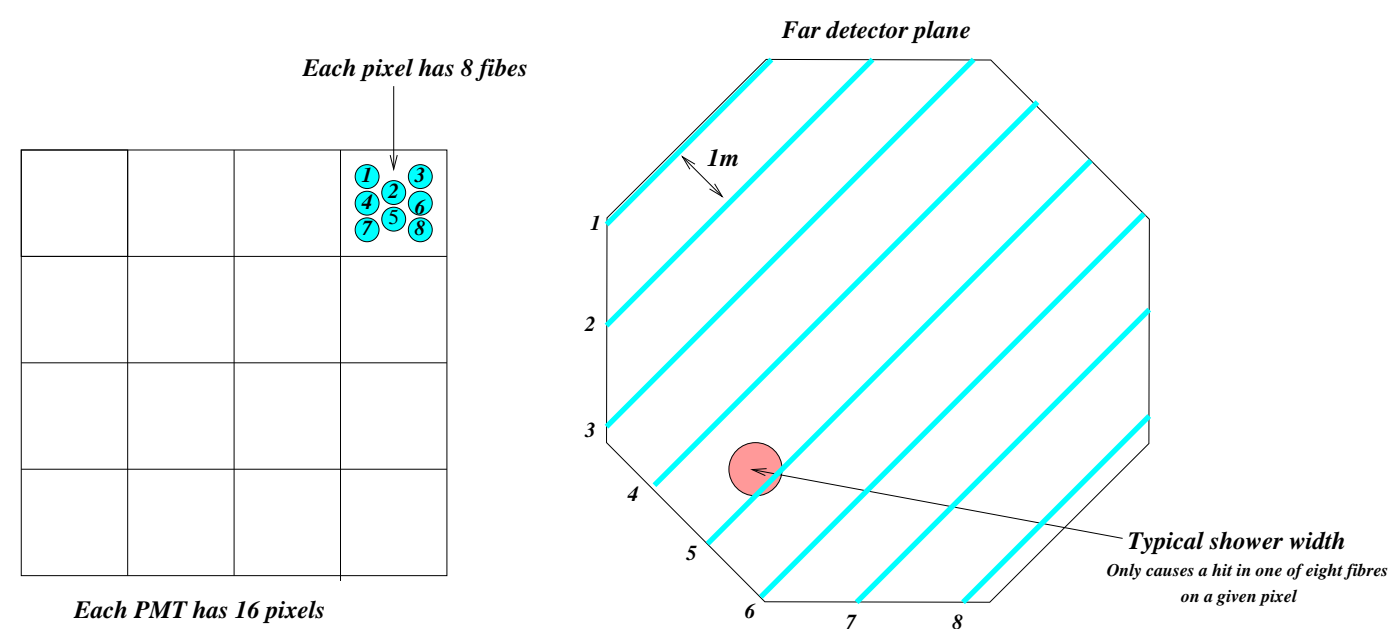

Figure 4.8: The multiplexing system employed at the far detector. Each PMT has eight fibres leading to each pixel, and each of these eight fibres leads from a strip in the far detector that is at least $1 \mathrm{~m}$ from any other strip illuminating the same pixel. The spacing is then wider than any typical shower size, as shown.

each PMT. The front end boards also contain 2 PIN photo diodes for light-injection calibration purposes.

Along the mid-level of the detector on both sides are VME readout crates. These are controlled by single board CPUs running VxWorks. This enables each VME crate to control and receive signals from 32 MUX boxes, thus in turn, reading out one side of 64 planes. There are $8 \mathrm{VME}$ crates per side of the detector to enable full coverage. Each VME crate also contains a timing PC card which timestamps events using a high-precision time source. Along the mid-level of the detector are crates containing programmable LeCroy 1440 high-voltage supplies for the PMT tubes, and "pulser boxes" which contain LEDs for light-injection calibration.

\subsubsection{Near Detector}

The aim of the near detector is to provide a reference for the neutrino beam at the point of its source, at Fermilab. Therefore the detector is situated only $1 \mathrm{~km}$ from the 


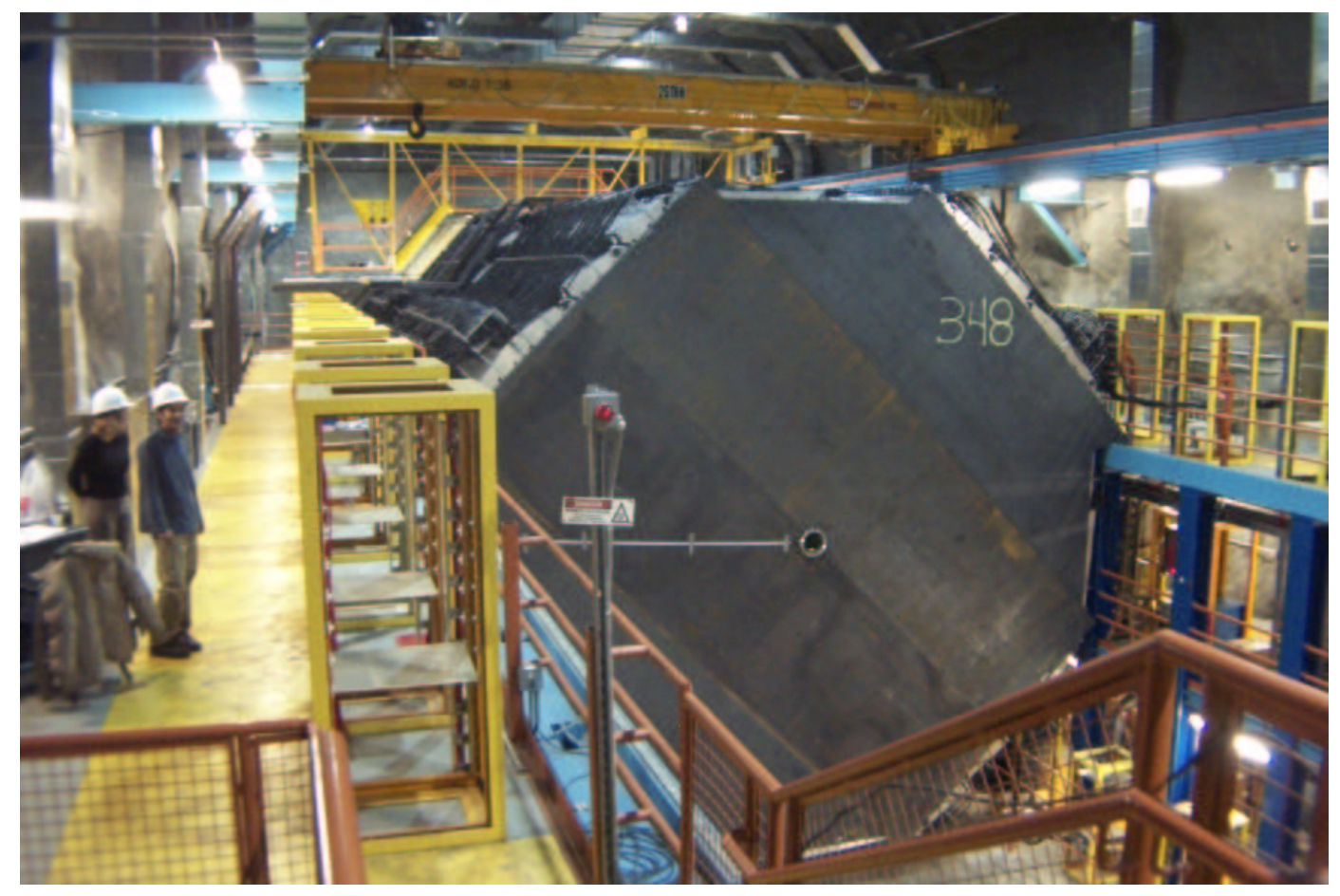

Figure 4.9: A photograph of the far detector during construction. The detector is built up to plane 348 at this point. The black conduit for the readout cables is clearly visible on the top left of the detector. Also the hole for the magnetic coil is present in the centre of the plane.

target hall and $100 \mathrm{~m}$ underground. In order to reduce systematic errors, the near detector is designed to be almost identical to the far detector. There are however several major differences in the construction of the detector.

The detector has a slightly different base shape. It is an elongated octagon, 3.8 $\mathrm{m}$ in height and $4.8 \mathrm{~m}$ in width. It is constructed with the same steel-scintillator-air design as the far detector, albeit with a few instrumentation differences which will be discussed. The detector is magnetised with a $1.5 \mathrm{~T}$ field. The hole for the coil is situated $0.5 \mathrm{~m}$ from the centre of the detector. The beam at the near detector only has a radius of $25 \mathrm{~cm}$ and is offset by $50 \mathrm{~cm}$ from the centre of the detector, on the opposite side to the coil. There is therefore a $1 \mathrm{~m}$ gap between the beam centre and 
the coil centre, as can be seen in figure 4.7.

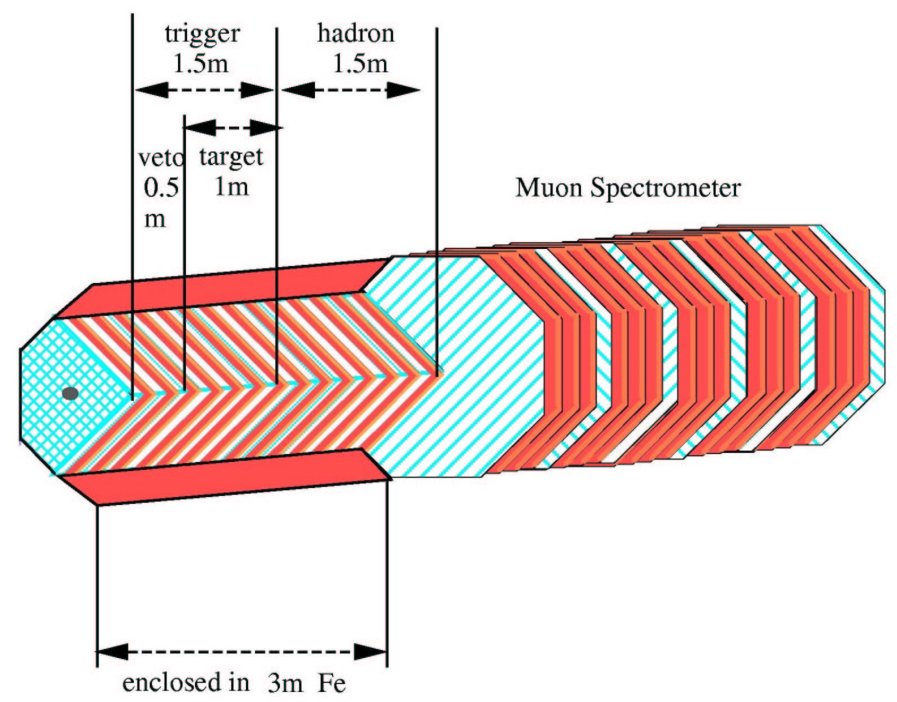

Figure 4.10: The near detector.

The near detector has a much higher neutrino flux than the far detector. Due to this having a high fiducial volume is not as important. To reduce cost and to improve data collection, the detector is divided into four sections as shown in figure 4.10. The first three sections make up the hadron/em shower calorimeter section of the detector, while the last section is intended for muon tracking.

- Veto Region - The first twenty planes of the detector are a veto region. They are to detect muons formed in the rock in front of the detector. Neutrino events here are ignored. This reduces possible end effects and ensures there is no background from neutron events. As with the next two sections which make up the hadron/shower calorimeter, this section only has one quadrant instrumented because the beam profile is so small. However, to keep track of low energy muons, which can wander out of the small region, every fourth plane is fully instrumented. 
- Target Region - The next 40 planes are to detect the vertex of the neutrino event. Only events that have the vertex here will be used for comparison with the far detector.

- Hadron Shower Region - 60 planes are dedicated to measuring the hadronic shower energies from interactions. To contain all hadrons from interactions formed within the target area, the hadronic shower region has a total thickness of $1.5 \mathrm{~m}$.

- Muon Spectrometer - The final 160 planes are designed to act as the muon spectrometer. As this section does not need to act as a calorimeter, only every fifth plane is instrumented. In this section however, the instrumentation extends over the whole of the plane. This allows the muon charge and momentum to be measured from the curvature of the track. In addition, in low-energy events, the range of stopping muons gives a more accurate measurement of their momentum.

Unlike the far detector only one side of each strip in a plane is read out. The opposite end of each fibre is coated with a reflector to increase the light yield. Due to the lower number of channels, and the higher event rate compared to the far detector, there is also no multiplexing. Slightly different PMTs are also used at the near detector: M64 tubes, also from Hamamatsu, have 64 pixels rather than the M16's 16 pixels. There are a total of 215 PMTs required to instrument the entire detector.

Due to the high event rate, a fast QIE [60] electronics system is used for the PMT readout. This is a multi-ranging ADC system in which dead time is eliminated by seperating each event into $19 \mathrm{~ns}$ buckets, which are individually digitised. These individual data sets are then recombined during reconstruction to reform the whole event. These electronics are very well understood, and therefore the systematic difference that they introduce to the experiment will be slight. 


\subsubsection{Calibration Detector}

The calibration detector (CalDet) allows the behaviour of muons, electrons and hadronic showers to be investigated within the MINOS detector structure. By placing the detector in test beams of known constitution these properties can be measured. The calibration detector is also used to refine the calibration techniques that are used for the near and far detectors. To these ends, the calibration detector was designed to be representative of a segment of the other detectors. Its size is such that it should contain showers of the size expected to be generated by neutrino events.

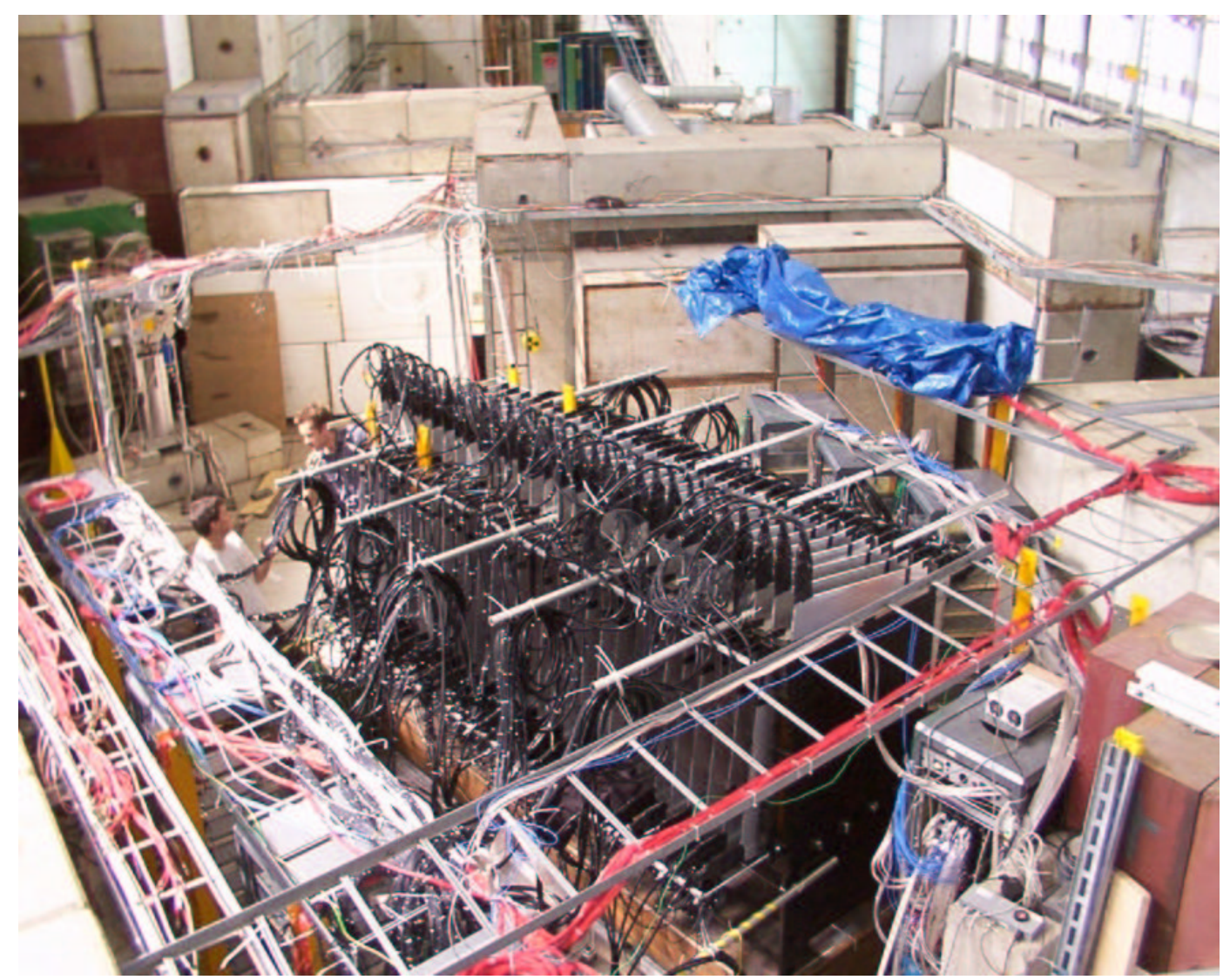

Figure 4.11: A photograph of the calibration detector in the CERN T11 beam area. Note the alternating planes at $90^{\circ}$ to each other, revealed by the black cabling conduit.

The CalDet is comprised of 60 planes, each $1 \mathrm{~m}$ square. As in the other detec- 
tors, alternate planes are rotated by $90^{\circ}$, as shown in figure 4.11. Each plane is fully instrumented with 24 strips of scintillator. The $6 \mathrm{~cm}$ pitch between planes is the same as that for the other detectors. As in the far detector, both sides of CalDet are read out. To help simulate conditions at the other detectors, one side of each plane is cabled with $6 \mathrm{~m}$ of clear optical fibre, while the other is cabled with $6 \mathrm{~m}$ of the green WLS fibre used in the modules. This helps simulate the longer fibre lengths within the modules used in the far detector.

These cables are fed into MUX boxes, where they are coupled to multi-anode PMTs. No multiplexing takes place. To date the front end electronics used have been the same as the far detector, with M16 PMTs. In the future, these will be replaced on one side of the detector by near detector M64 PMTs and QIE electronics. This will allow the response of each system to be directly compared.

As the far-detector electronics were designed for a very low event rate, they could not cope with the high flux of particles from the test beam. The result of this at the CalDet is that after each event, the DAQ has an effective dead time of $\sim 5-10 \mu s$. Events that fall within this time region are discarded.

Over the past two years, CalDet has been placed in two different beam areas in the East Hall of the CERN PS system. The beam lines used have been T11, which has energies from 0.5-3.5 GeV, and T7, which has energies from 3.5-10 GeV. Both beams contain a mix of $\pi, p, e^{-}$and $\mu$; these latter are generated from pion decay. The beam spill lasts $\sim 0.5$ seconds.

To enable particle identification in the calibration detector there are two Time Of Flight (TOF) counters, and three Čerenkov detectors placed within the beam line, as shown in figure 4.12. The TOF counters enabled $\pi$ and $p$ separation up to $3 \mathrm{GeV}$; an example is shown in figure 4.13. Above this energy the $\pi$ and $p$ identification was performed by the C̆erenkov counters. The C̆erenkov detectors were capable of identifying electrons at all energies. 


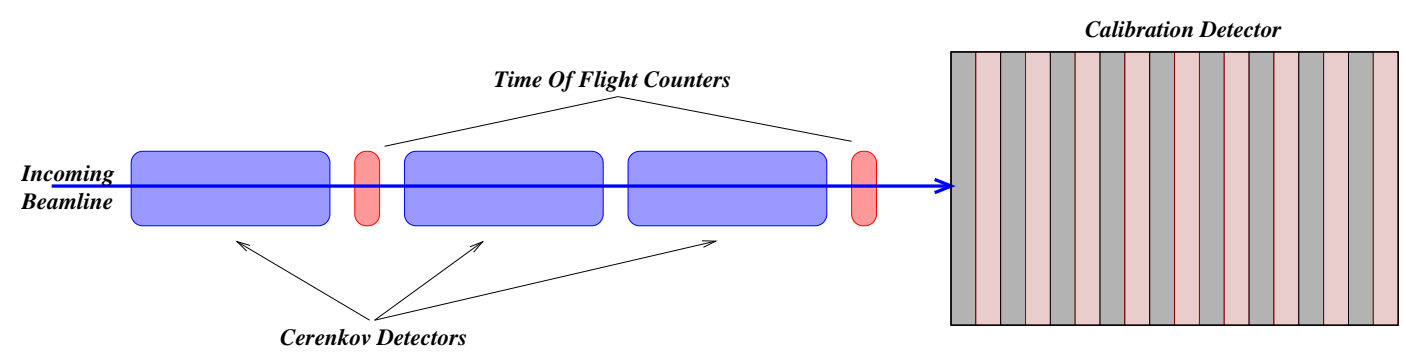

Figure 4.12: A schematic of the calibration beam line. Three argon Čerenkov detectors and two TOF counters are used.

\subsection{Physics Capabilities}

Neutrino events within the MINOS detector can be classified under three groups: so-called long Charged Current (CC) and short Charged Current (short) events, in which a W boson exchange takes place; and Neutral Current (NC) events, in which a $\mathrm{Z}$ boson exchange takes place. These are illustrated in figure 4.14. The ratio of these short and long events as a function of energy can be used to identify $\nu_{\mu} \rightarrow \nu_{\tau}$ oscillations. This is known as the $T$-test:

$$
T=\frac{(\text { short } / \text { long })_{\text {near }}}{(\text { short } / \text { long })_{f a r}} .
$$

This method removes some of the systematics due to the differing rates at the near and far detectors, as they cancel out in the ratio. The measurement is sensitive to oscillations both via the reduction in long $\mathrm{CC}$ events and via the corresponding increase in short events as the $\nu_{\mu}$ in the beam are replaced by other flavours. Figure 4.15 shows the sensitivity of MINOS from using the $T$-test after two years of running.

The $T$-test is vulnerable to a systematic error in the number of $\mathrm{NC}$ events recorded. As the cross section for NC events rises sharply at low energies, it is very important to have as accurate a calibration as possible. The lower in energy that we can detect and accept the NC events, the more statistics we will have for the $T$-test measurement. 

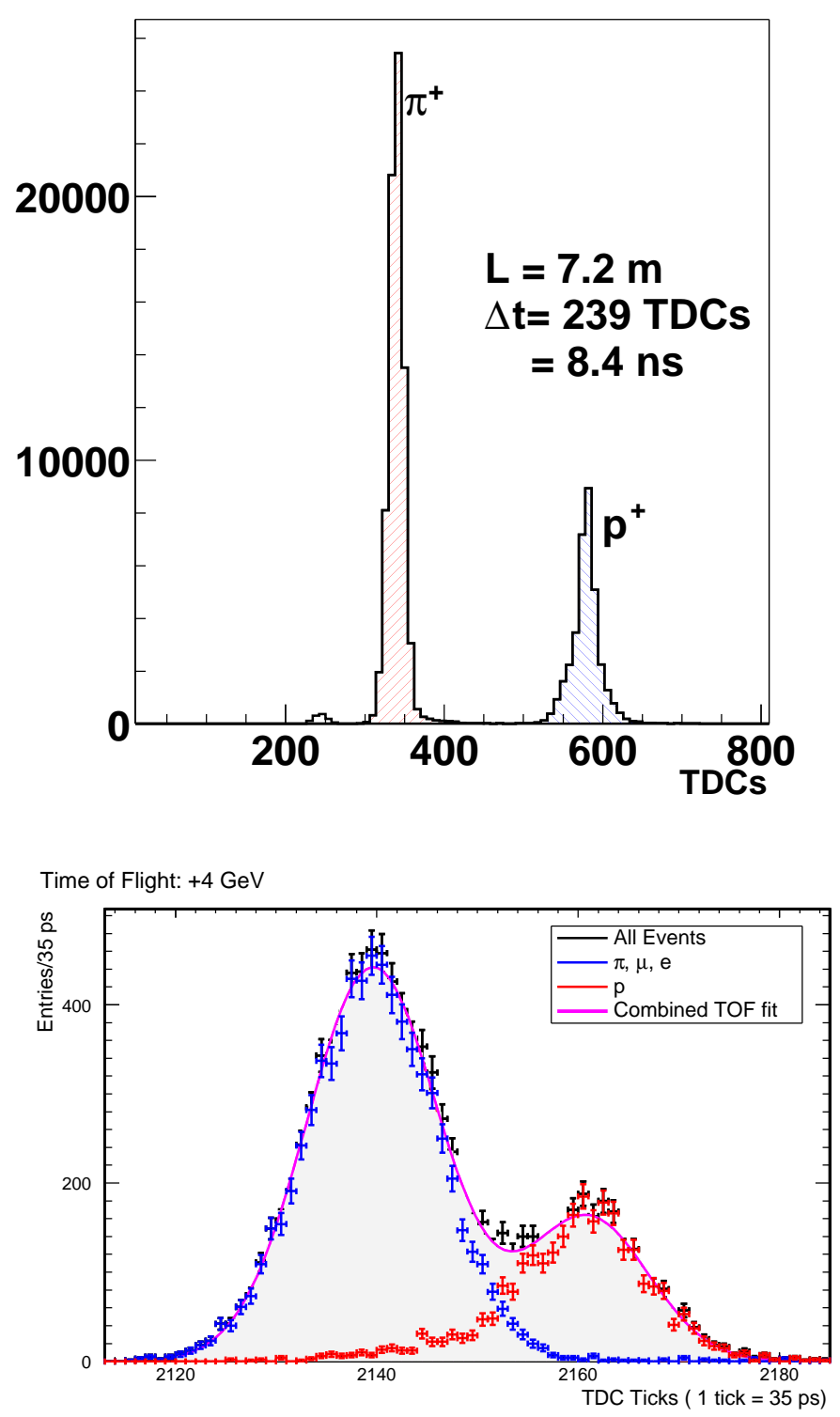

Figure 4.13: TOF separation at the calibration detector. The top plot shows the very clear separation at $1 \mathrm{GeV}$. The bottom plot shows the limit of the separation capabilities at $4 \mathrm{GeV}$. 

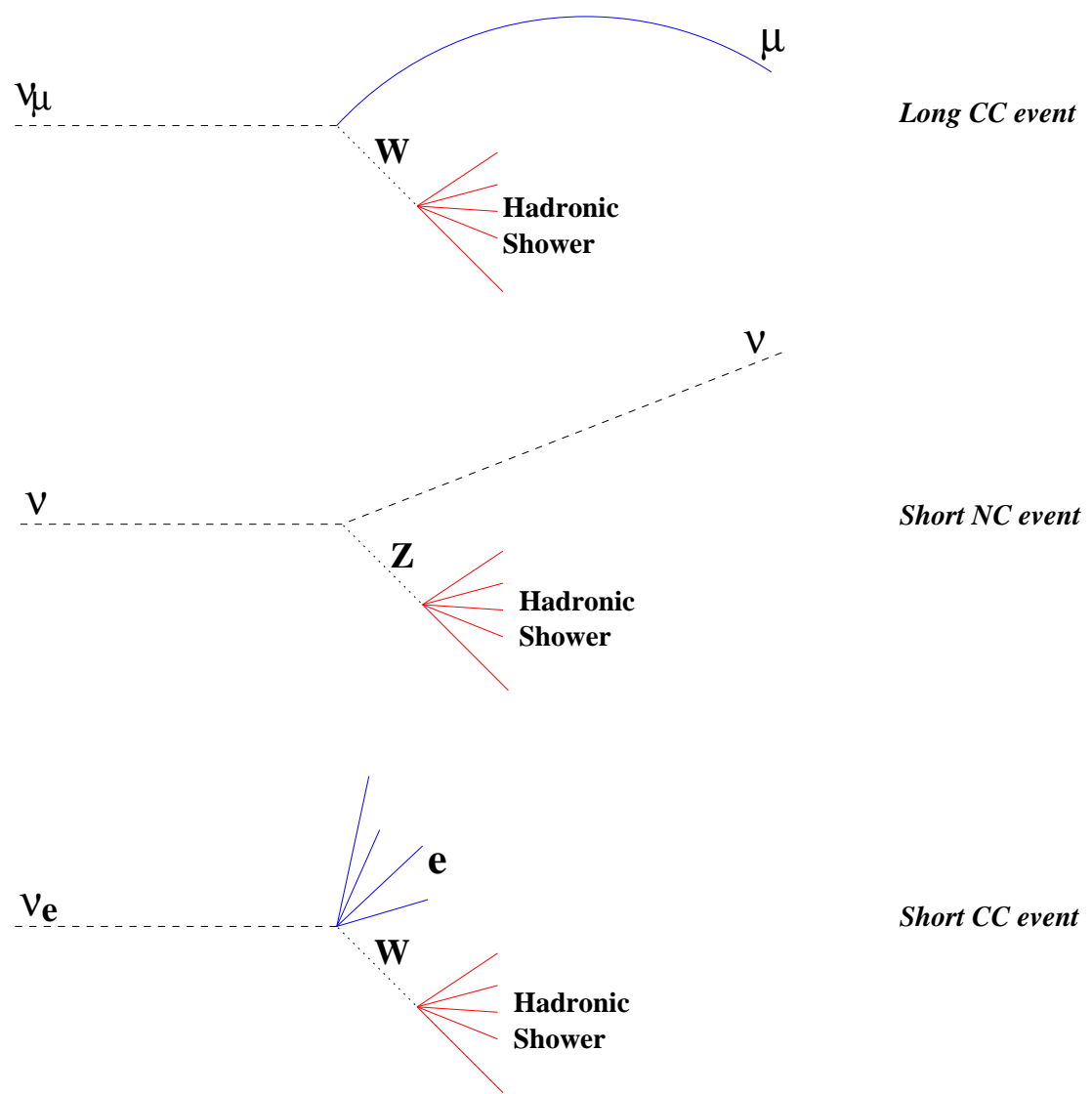

Figure 4.14: Diagram showing the three main types of neutrino interaction that will be detected. $\nu_{\tau}$ events, with high enough energy, will look like long $\mathrm{CC}$ if the tau decay yields a high-energy muon; otherwise it will appear as a short $\mathrm{CC}$ event.

The second oscillation measurement available to the MINOS detector arises from measurement of the CC energy spectrum. Figure 4.16 shows simulations of this method to detect oscillations under three scenarios. This technique works by extrapolating the near detector spectrum to predict the far detector spectrum, as if there were no oscillations. A depletion of $\mathrm{CC}$ events at particular energies should then appear if oscillations take place. The position of the dip in the $\mathrm{CC}$ spectrum is defined by $\Delta m^{2}$, while the fractional amount of depletion is governed by $\sin ^{2} 2 \theta$. As this method is very dependent upon the energy of the events, the absolute energy calibration between the detectors is extremely important. Also important is 
the Monte Carlo used to extrapolate the near detector spectrum to the expected far detector spectrum. Figure 4.17 shows the sensitivity of MINOS to CC spectrum oscillations after two years, running with maximal mixing (i.e. $\sin ^{2} 2 \theta=1$ ). 


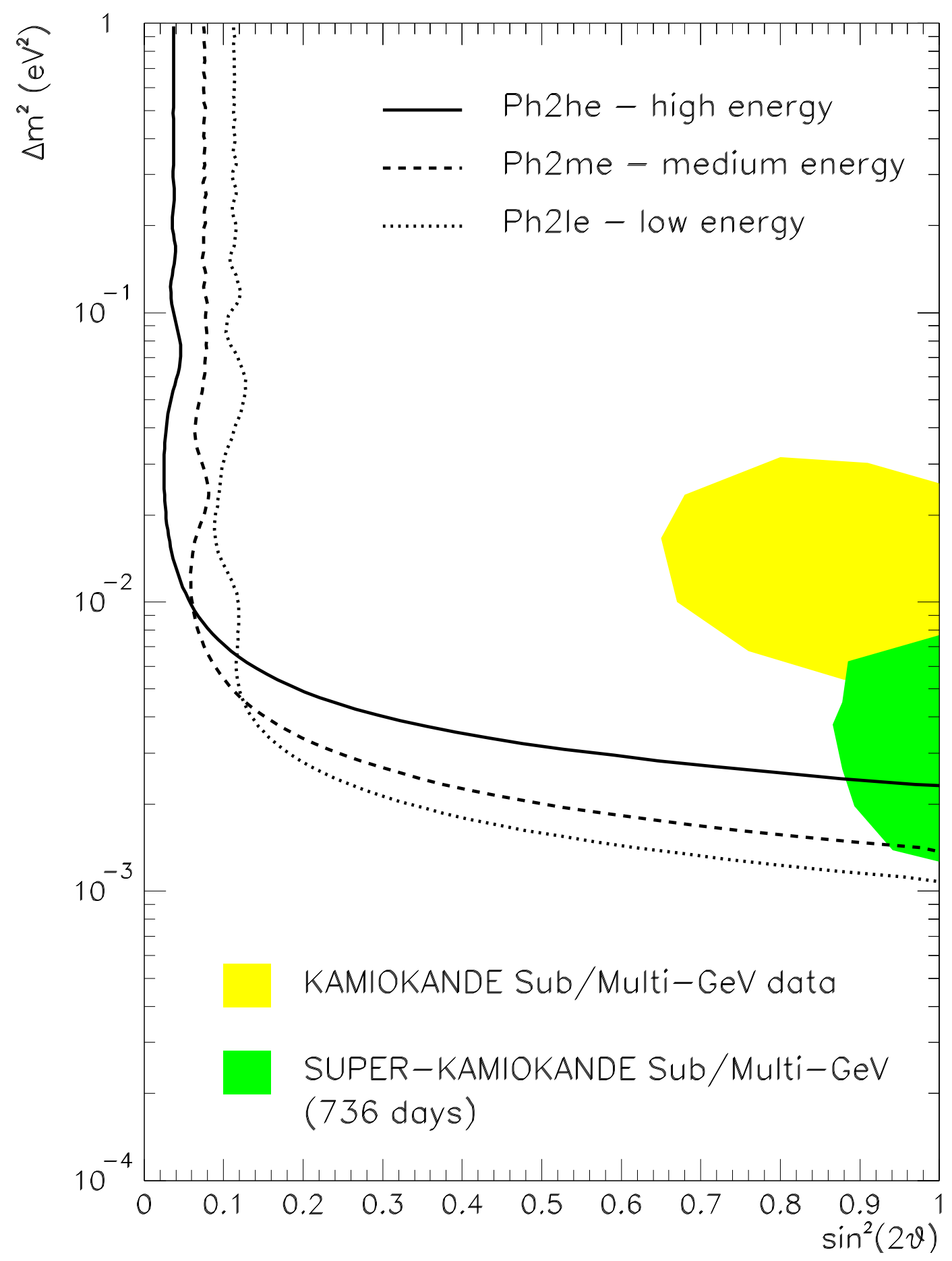

Figure 4.15: This plot shows the region of phase space covered to $90 \%$ exclusion by the T-test for $\nu_{\mu} \rightarrow \nu_{\tau}$ after two year's running. Also shown are the regions favoured by the Super-Kamiokande results. The regions for the three possible beam energies are shown. Even though MINOS will run with the low energy beam initially. 
CC energy distributions - Ph2le, $10 \mathrm{kt} . y \mathrm{r} . \sin ^{2}(2 v)=0.9$
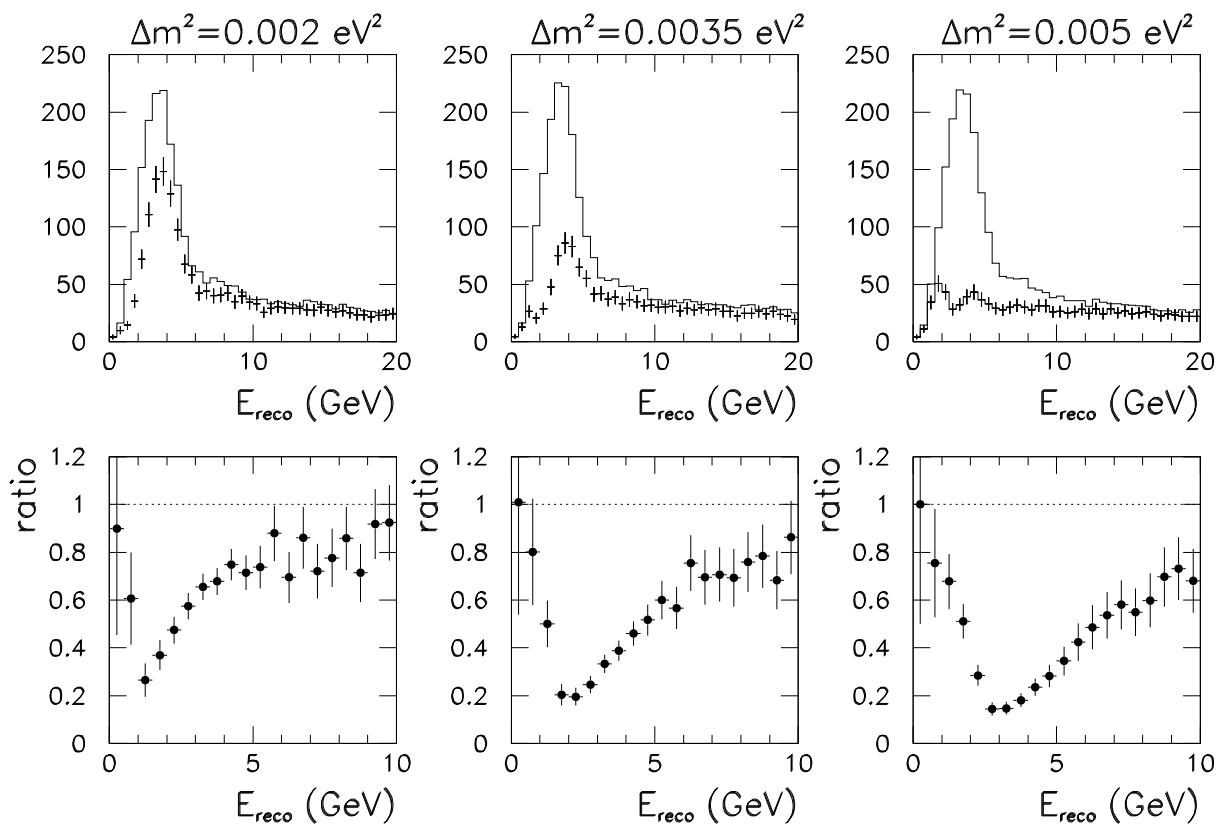

Figure 4.16: Energy spectrum produced by oscillations. The top three plots show the reconstructed energy distributions with and without oscillations. All plots assume $\sin ^{2} 2 \theta=0.9$. The solid histograms show the unoscillated distributions, the points with error bars are the oscillated distributions. The bottom plot shows the ratio of these. All plots are for $10 \mathrm{kt}$ year exposure, with the low energy beam. 


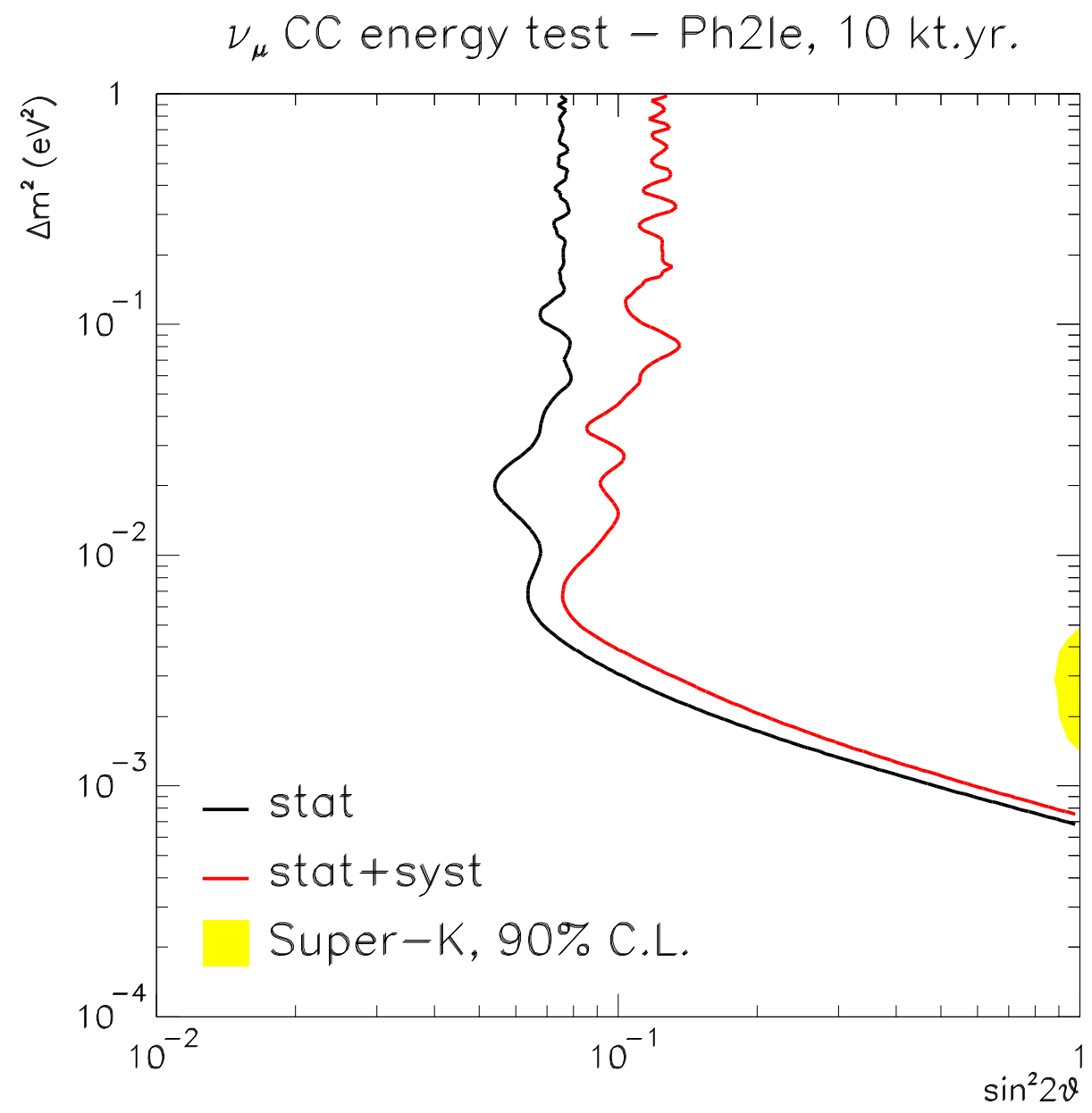

Figure 4.17: $90 \%$ exclusion limits for two years of CC spectrum oscillation analysis, assuming maximal mixing and no oscillations are observed. (The SuperKamiokande results are shown for comparison) 


\section{Chapter 5}

\section{Calibration}

\subsection{Calibration Methods}

The calibration of any detector is obviously very important, and greatly affects the resolution and accuracy of any results. The MINOS detectors especially need careful calibration, as the energy calibration between the two main detectors will greatly affect the measurements of the oscillation parameters.

The calibration of the MINOS system therefore requires that all three detectors should be calibrated to remove any internal variations. Each detector then needs to be calibrated relative to the others, to allow energy readings in each detector to be compared. Finally, these normalised energy responses from each detector need to be translated into physical energy units.

There are six main calibration techniques that can be carried out to optimise internal energy response of the detectors. Figure 5.1 shows a summarised calibration chain used to convert the raw ADC units integrated from the PMTs, into particle energies and types. 


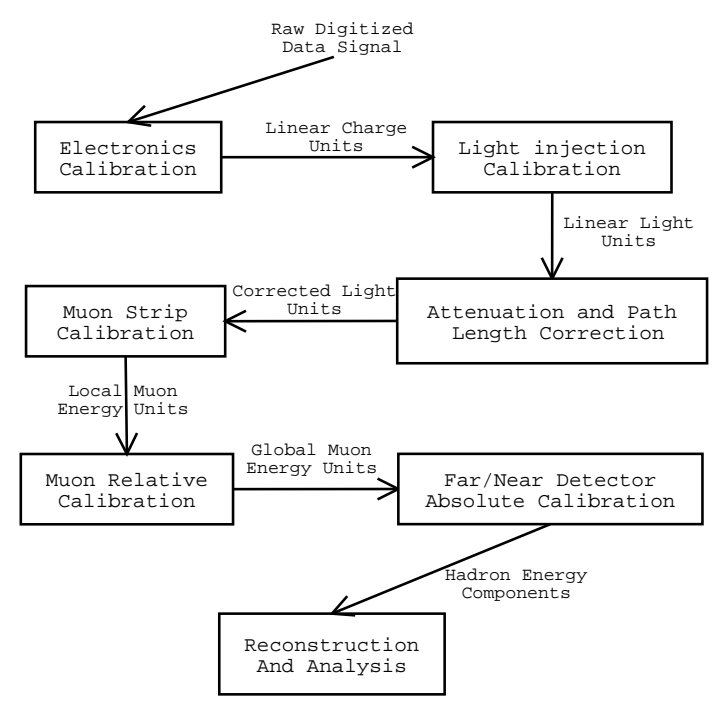

Figure 5.1: A schematic showing the calibration method used in MINOS.

\subsubsection{Charge Injection}

The electronics on the front end boards are calibrated by a method of charge injection. A digital potentiometer on the electronics board sets the amount of charge that is to be injected into the digitisation electronics. As the injected charge is known very accurately, this method is capable of calibrating the front end electronics to the level of $1 \%$ of full range. The simple single-range ADCs of the far detector only need a few points per channel to achieve this. The near detector QIE electronics have a more complicated electronics system, so require more data points per electronic channel to map the response.

\subsubsection{Light Injection}

The role of light injection in calibration is fourfold. It is designed to monitor any changes in the gain of the PMTs with time, as well as to measure the non-linearity of the PMTs at high light levels. It is also used to measure PMT gains, via single photo electron spectrum, and ensures integrity of the optical readout chain. The light-injection system consists of "pulser boxes" that contain blue LEDs. These 


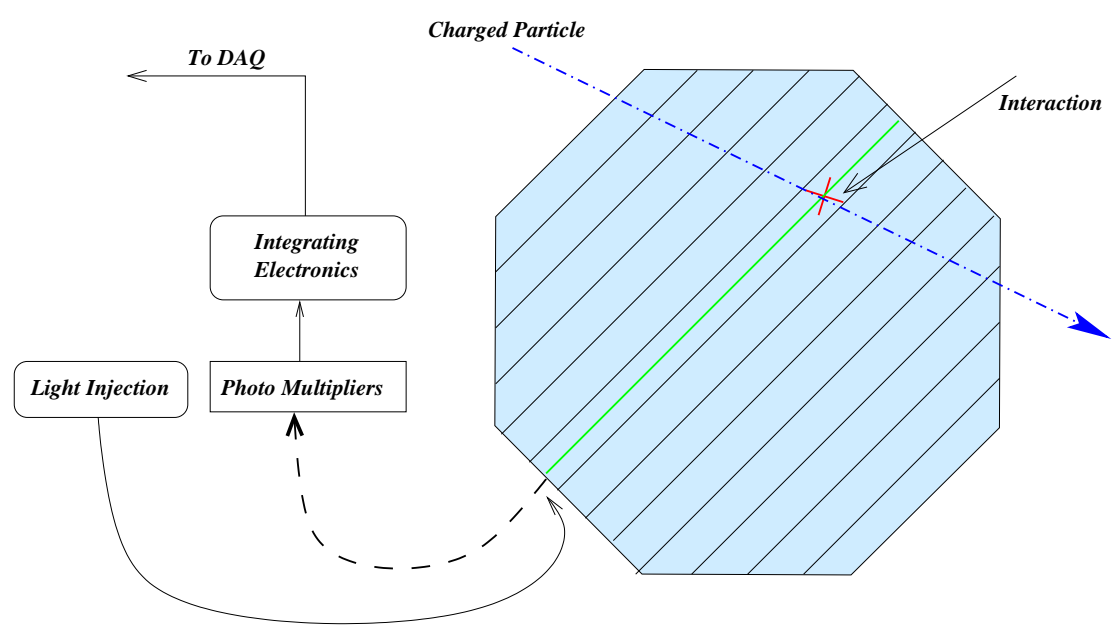

Figure 5.2: A schematic showing the readout chain of a signal produced by a charged particle interaction, and the light-injection system entry point.

can be pulsed for very short durations to mimic the light deposited from a charged interaction within the scintillator. This light is "injected" into the readout chain via Light Injection Modules (LIMs). The light from the pulser system can then be read out by the normal front end electronics. The design and testing of the light injection system formed a major part of my PhD studies. I have therefore devoted chapter 6 to a detailed discussion of the light injection system.

\subsubsection{Attenuation and Path Length Correction}

The amount of light received by any PMT from an event is directly affected by the length of fibre the signal has passed though. Therefore an attenuation correction is made to the data depending upon the position of the interaction. The hits are corrected for as if they all passed through the centre of a strip. As there is no reflective material on the end of each strip, light is lost into the manifold at the extremities of each strip. A sample of cosmic muons are used to measure this behaviour for each strip. This data is then fitted to calculate a correction function according to position along the strip, which can be calculated by looking at the next strip which is rotated 
by $90^{\circ}$. Figure 5.3 shows an example of this.

A path length correction is also made depending on the angle the particle enters the scintillator strip. Generally particles with a higher angle of attack to the scintillator strips will deposit more light due to the longer path length. All hits are therefore corrected as if they had passed orthogonally through the strip.

\subsubsection{Strip-to-Strip Calibration}

Calibration can also be preformed on a strip-to-strip basis within each detector by using cosmic muons. As the muons will deposit a known amount of energy per unit length travelled in the scintillator, they can be used to find the relative strip response within the detector. It is not possible however to use this method to cross-calibrate the detectors, as each detector see muons of varying energies. The cosmic muon energy spectrum rises sharply at the surface, so the near detector will mainly see low energy muons of a few GeV. However the far detector, as it is very well shielded with rock, will only receive muons with energy of the order of $100 \mathrm{GeV}$ or more. Simulations have shown that there will be enough muons detected within a period of one month to calibrate the near and far detectors to $0.5 \%$ and $2.4 \%$ respectively. Higher accuracy than this is not necessary, as there will be a large statistical error on the strip-to-strip response of a particle interaction, .

\subsubsection{Stopping Muons}

Muons can also play a second part in the calibration of the minos detectors. The energy of a stopping muon can be measured to within $6 \%$ by looking at its range. Each detector will receive a large number of stopping muons per day: $\sim 700$ in the far detector and two orders of magnitude more than that in the near detector. By studying the response of the detectors to these muons of known energy we can calibrate the two large detectors, and the calibration detector, together in terms of 

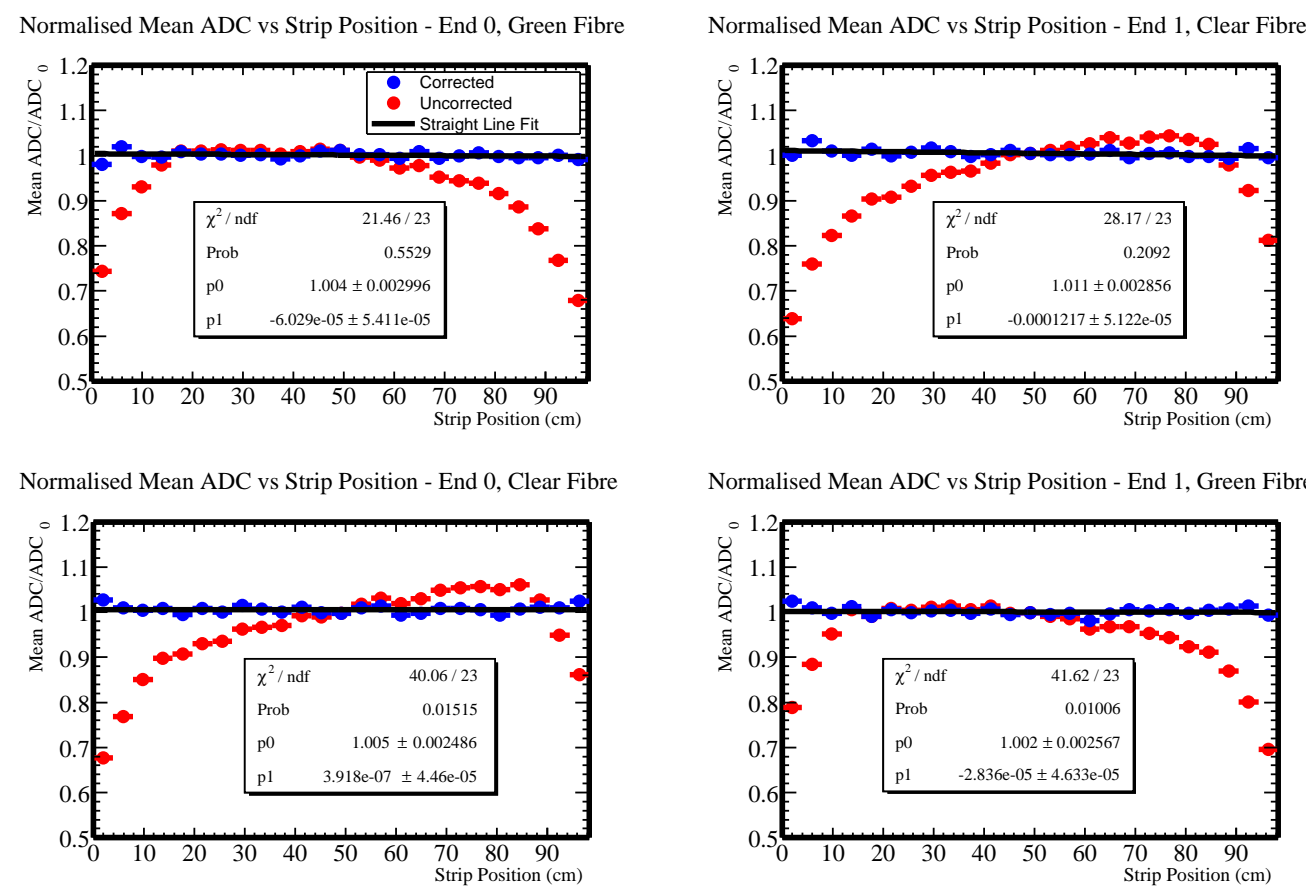

Figure 5.3: The dependence on muon pulse height with position along a scintillator strip with and without corrections, from [61]. There are two strips shown here, with the readout from both ends shown for each. As expected the uncorrected curve is mirrored for each end. Each curve is characterised using a three quadratic terms, one for each of the three different regions: the central region where the light level drops smoothly with distance from the PMT, and the two edge regions where the light output drops suddenly due to light absorption from the manifold. This occurs because a large proportion of light dispersed from an interaction at the end of a strip will be absorbed by the manifold itself, before having a chance to be absorbed by the the WLS fibre. 
Muon Energy Units ${ }^{1}$ (MEUs) to within $1 \%$.

\subsubsection{Absolute Calibration}

The calibration detector is designed to relate the response of the detector technologies to hadronic and electromagnetic showers to the known responses of muons. By subjecting the calibration detector to particles from CERN test beams of known energies, the response of the detector can be measured. The muons from these beams can then be utilised to transfer this energy knowledge to the far and near detectors. The response from the hadrons, electrons and other particles from the test beam can then be translated into muon energy units (MEUs) and transferred to the other detectors. This will provide the final calibration step needed to translate the light output from an interaction within the detector into physical energy units. The design of the calibration detector will allow it to perform this absolute calibration to a level of $5 \%$.

\subsection{Calibration Detector Results}

\subsubsection{Particle Identification and Response}

By using the particle identification detectors the response of the MINOS technologies within the calibration detector has been successfully measured. Figure 5.4 shows the different responses of the calibration detector to $\pi$ and $p$. The energy resolution of the calibration detector has proved to be as expected. Figure 5.5 shows the energy resolution for $\pi$ and $p$. Above $1 \mathrm{GeV}$ this resolution is approximately 0.56 as shown in figure 5.5. This information is very useful for fine-tuning the Monte Carlo simulations, which will be used in the final CC analysis of the MINOS data.

\footnotetext{
${ }^{1}$ A Muon Energy Unit is the energy deposited by a muon passing through a known length of scintillator, a useful baseline measurement for comparing the responses of the detectors .
} 


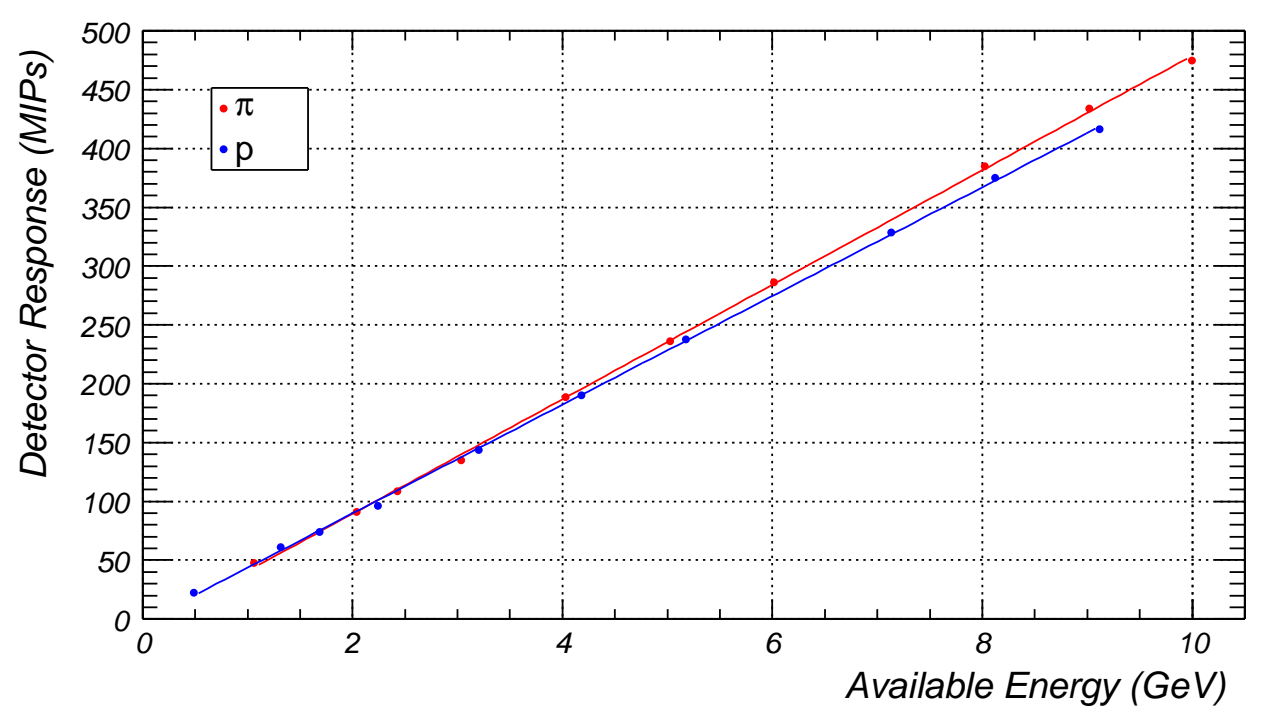

Figure 5.4: Calibration detector energy responses for $\pi$ and $p$.

Similar analysis has been done with electrons. Figure 5.6 shows the detector response and resolution for electrons.

These calculations of detector response form a very important part of the MINOS calibration. By transferring these response profiles to the near and far detectors, deposits from particle interactions at these detectors can be represented as energy. This provides us with a method of measuring the energy deposits of interactions as required. 

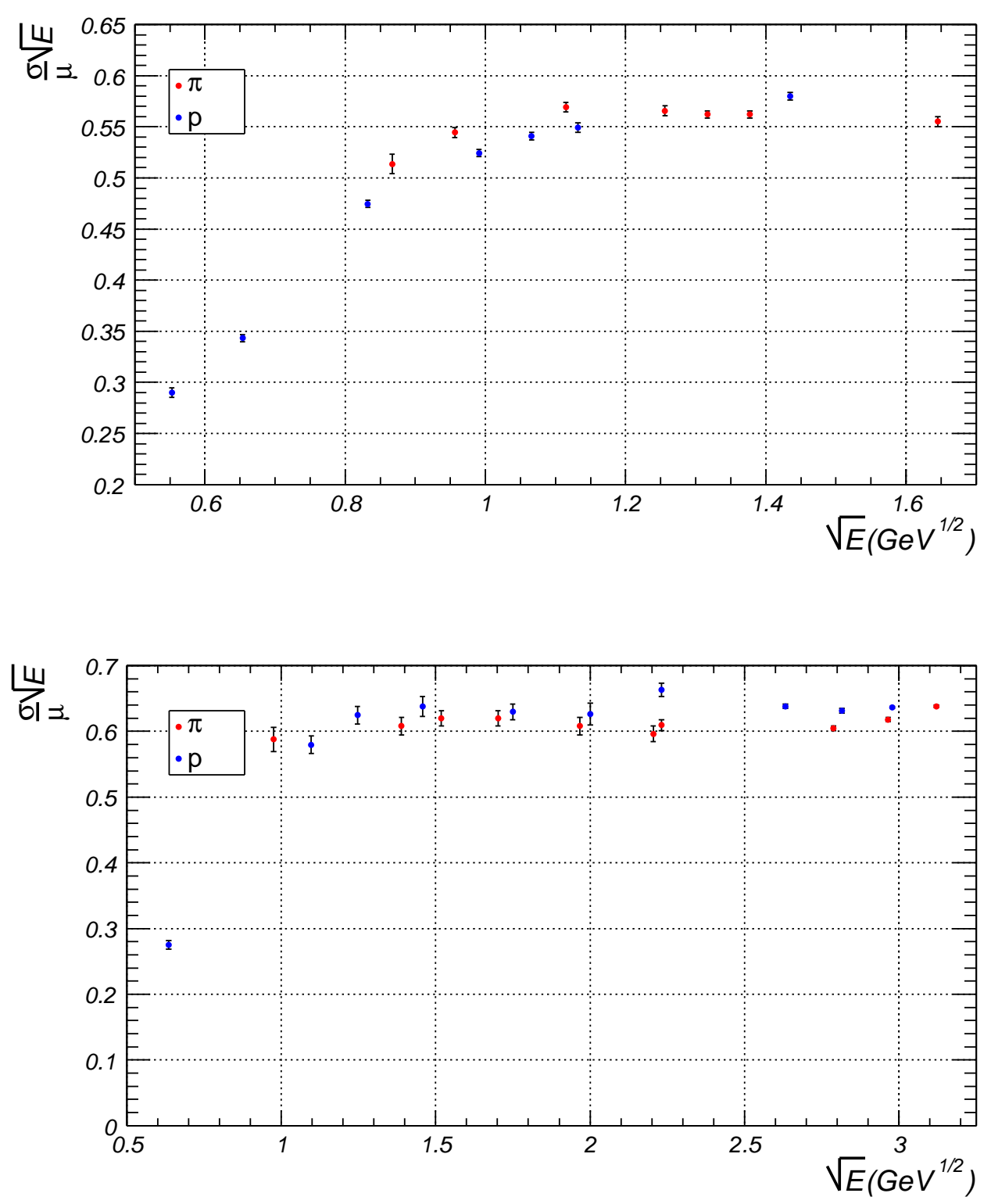

Figure 5.5: These two plots show the hadronic energy resolution of the calibration detector. The top plot shows different data taken covering just the lower energies. 

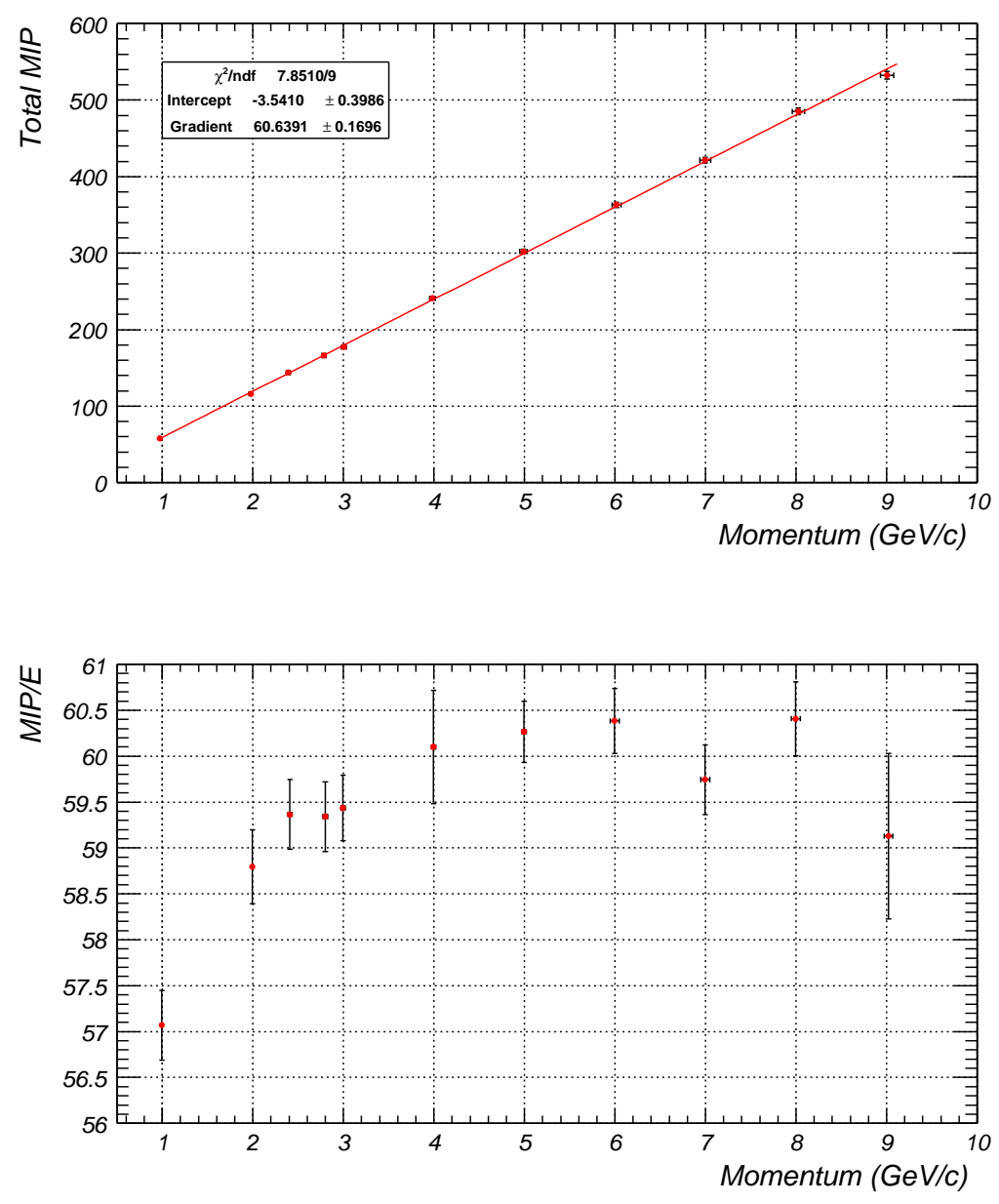

Figure 5.6: These plots show the response of the calibration detector to electrons, as a function of momentum. The energy resolution is also shown 

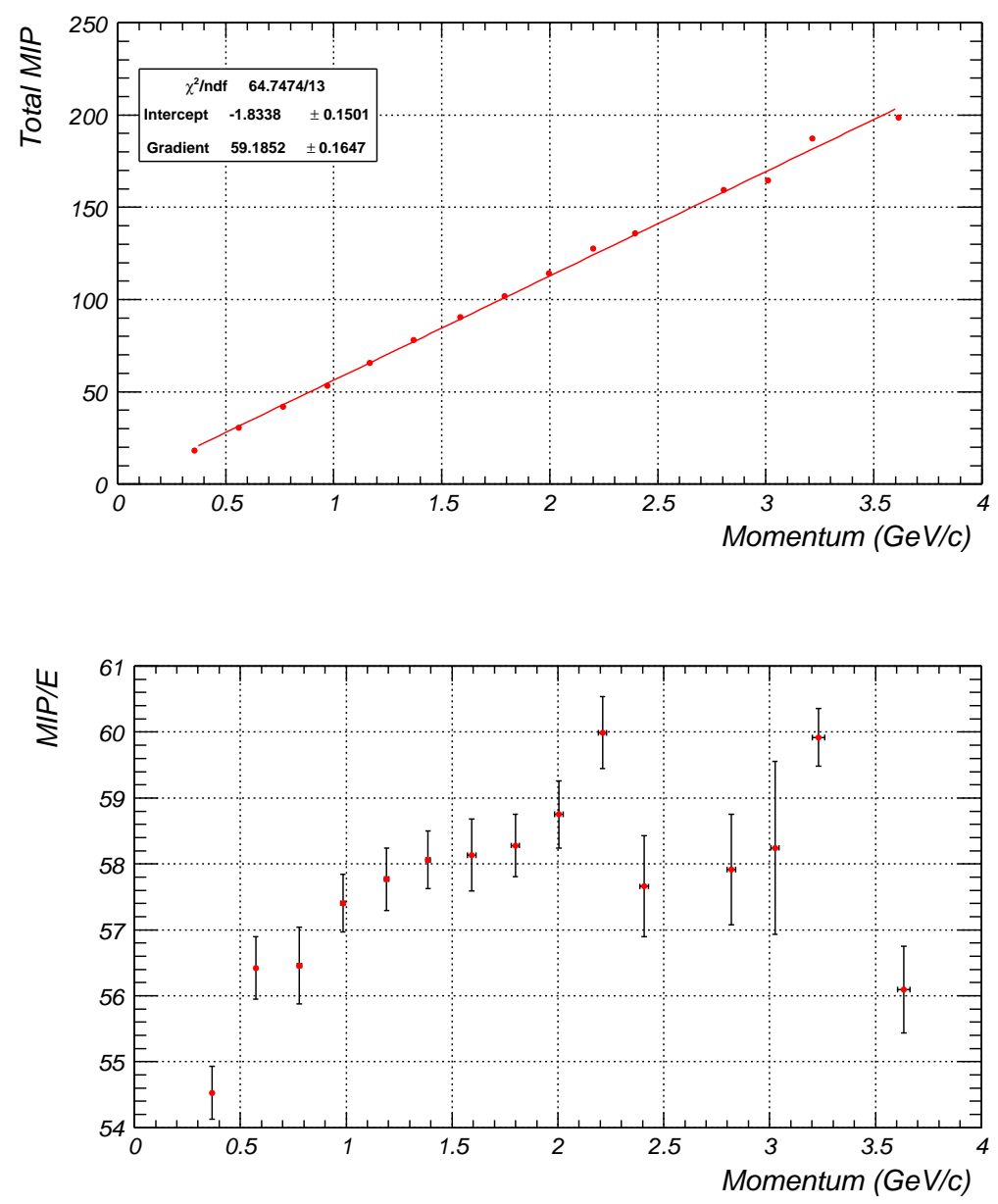

Figure 5.7: Similar to the previous plots, in figure 5.6, these plots show the response of the calibration detector to electrons, along with the energy resolution. However on this plot only the lower momentum range is shown for clarity. 


\section{Chapter 6}

\section{Light Injection System}

\subsection{Light Injection Aims}

The aim of the light injection system is fourfold:

- Mapping the gain curve - The PMTs are only linear at low light levels, below about 100 photoelectrons [62]. The light injection system is to be used to characterise the linearity of each photomultiplier channel.

- Monitoring PMT gain drifts - photomultiplier gains can be sensitive to temperature and voltage variations. By pulsing the system on an hourly basis, these gain changes can be measured and taken into consideration during reconstruction.

- Gain Calculation - By pulsing the system at very low light levels, the response spectrum from the PMTs can produce a single photoelectron peak, as in figure 6.1. This peak can be used to calculate the gain of each PMT.

- Optical path integrity - Beyond the scope of calibration issues, the light injection system can also be used to check the integrity of the entire readout 


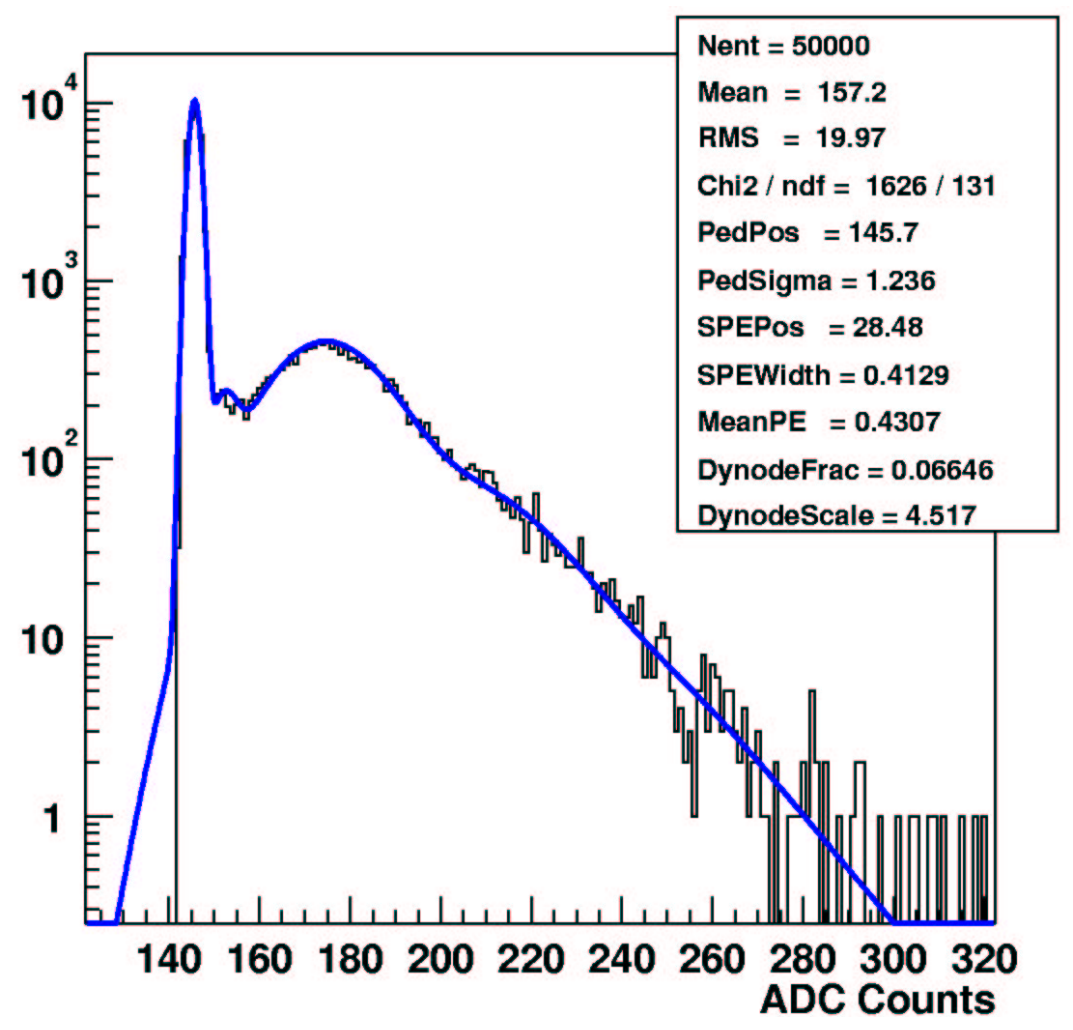

Figure 6.1: A typical plot of the single photoelectron peak. Shown is a fit from the algorithm used to calculate the gain of the PMTs; this plot corresponds to a light level of 0.43 photoelectrons. [63]

system. As the light injection system flashes every fibre, broken or wrongly connected fibres are easily identified, and can be repaired. This is especially important during construction.

\subsection{Light Injection System Design}

The light injection system is based on pulsed Light Emitting Diodes (LEDs). These LEDs are situated in a rack-mounted crate known as a pulser box. Each pulser box supplies light to one side of 64 planes. There are normally twenty LEDs per pulser box. The LEDs are each coupled to 64 multi-way optical connectors on the rear of the pulser box via collections of optical fibre known as ponytails. The ponytails are 
knitted into these connectors such that each connector has one fibre from each of the LEDs. Optical cables are then attached to these connectors, and these carry the light from each LED to a separate Light Injection Module (LIM) on each plane. The LIMs incorporate $\sim 3 \mathrm{~cm}$ of the green WLS signal fibres at the end of the scintillator modules. The interior of each LIM is highly reflective, to maximise the amount of incoming light absorbed by the signal fibres. The light signals then follow the same readout path as real data signals, figure 6.2 shows a schematic of the system. This system of light injection allows controlled light pulses to be injected into the readout chain as if it were light from a particle interaction.

There were several constraints placed upon the design of the pulser system. These were to ensure the maximal performance of the calibration system:

- In the far detector the M16 PMTs are multiplexed, with eight fibres carrying light to each pixel. No two fibres on a pixel must be illuminated simultaneously. Also, to reduce crosstalk, no two edge-adjacent pixels may be illuminated simultaneously.

- The dynamic range of the system should be such that it can supply a range of light levels from a fraction of a photoelectron to several hundred photoelectrons. It must be able to achieve this with a resolution of one photoelectron.

- The relative light levels injected into the PMTs by the LEDs must be monitored by a separate device to measure non-linearity. PIN photo diodes were selected for this purpose. There are two PIN photo diodes for monitoring each LED, one with a gain of roughly twice the other. There are several reasons for having two PINs:

- It allows one to maintain the calibration even if changing the front-end board on which a PIN is mounted, the other PIN provides a reference point to calibrate the new PIN. 


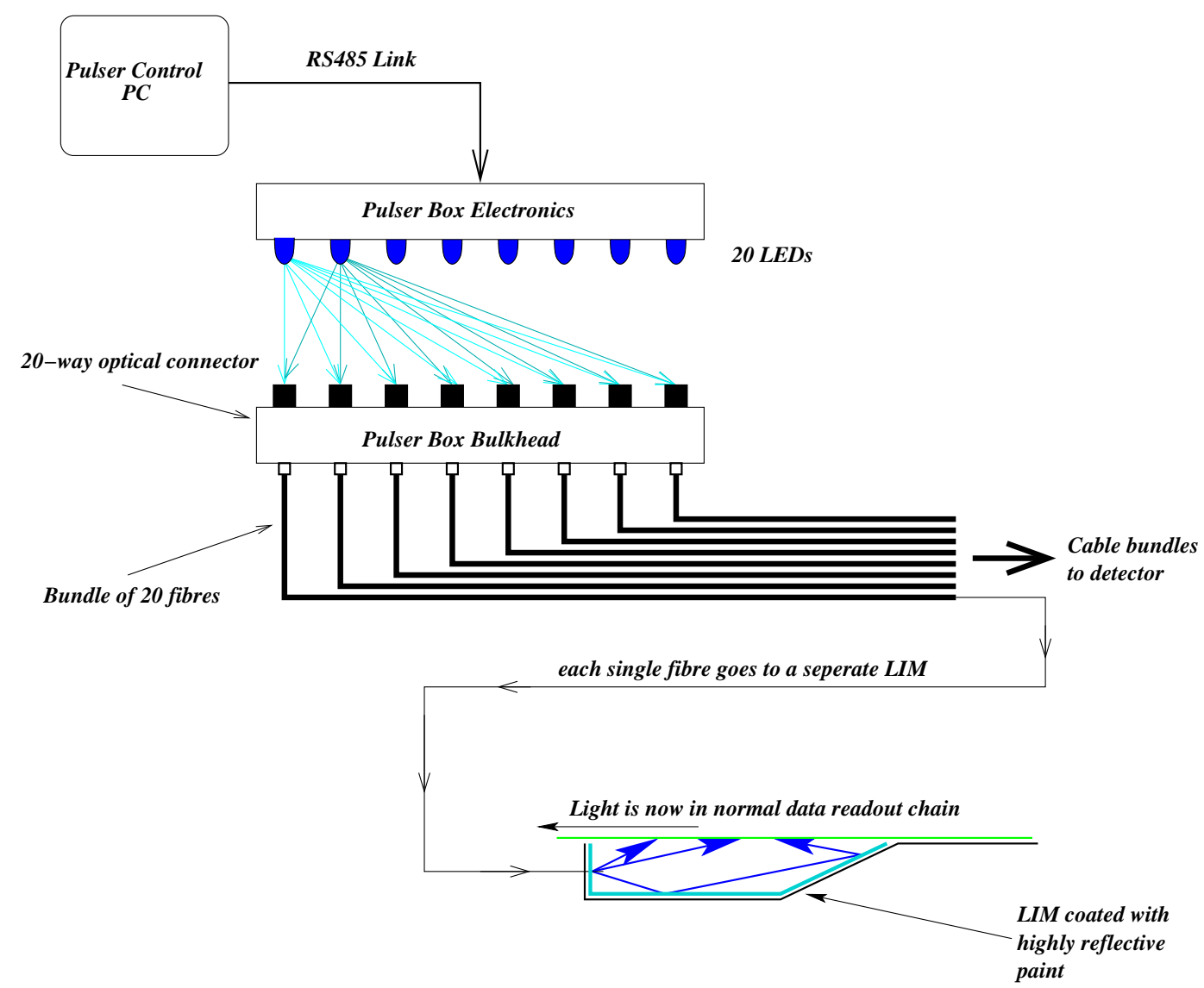

Figure 6.2: A schematic of the light injection system. Only a small number of the components for each section, and the fan out from only two LEDs, are shown for clarity. The light from each LED is shared between 70 bundles of 20 fibres, with each fibre from a bundle leading to a separate Light Injection Module. The distribution of these fibres has to be carefully organised to allow calibration data to be reconstructed. This is due to the multiplexing described at the beginning of chapter 4. 
- It provides a backup of the system in the unlikely event of a failure.

- The twin-gain option effectively doubles the resolution of the system: the high-gain PIN is particularly sensitive at low light levels, but may saturate the front end electronics at high light levels.

- The response of the independent linearity-monitoring PIN photo diodes must be stable to within $1 \%$ over the period of a month.

- The arrival times of the light pulses to each PMT must be stable to within one nanosecond.

- The amount of light on simultaneously illuminated pixels on a given PMT should differ by no more than a factor of two. This is important for two reasons; firstly, it helps to reduce excessive crosstalk between pixels, and prevents the problem of a bright pixel causing the other pixels to behave in a non-linear fashion. It also reduces the number of data points needed to cover the dynamic range of all pixels to the required resolution. This is explained in figure 6.3 .

\subsection{LEDs}

It was decided to use LEDs for light injection. Lasers were considered, and have several advantages over LEDs. Lasers are brighter by several orders of magnitude, and have a spectrum of light that closely mimics that of the scintillator. However, for MINOS the advantages of using LEDs outweighed these factors. It was found that the pulse to pulse variations from LEDs was very small, resulting in the response of the PMT being dominated by photon statistics. The LED system design is based on solid state electronics with no moving parts, and there are no consumable supplies such as dye or gas needed. Therefore the system is perfect for long term remote control. The pulsing light level of the system is controlled by software 


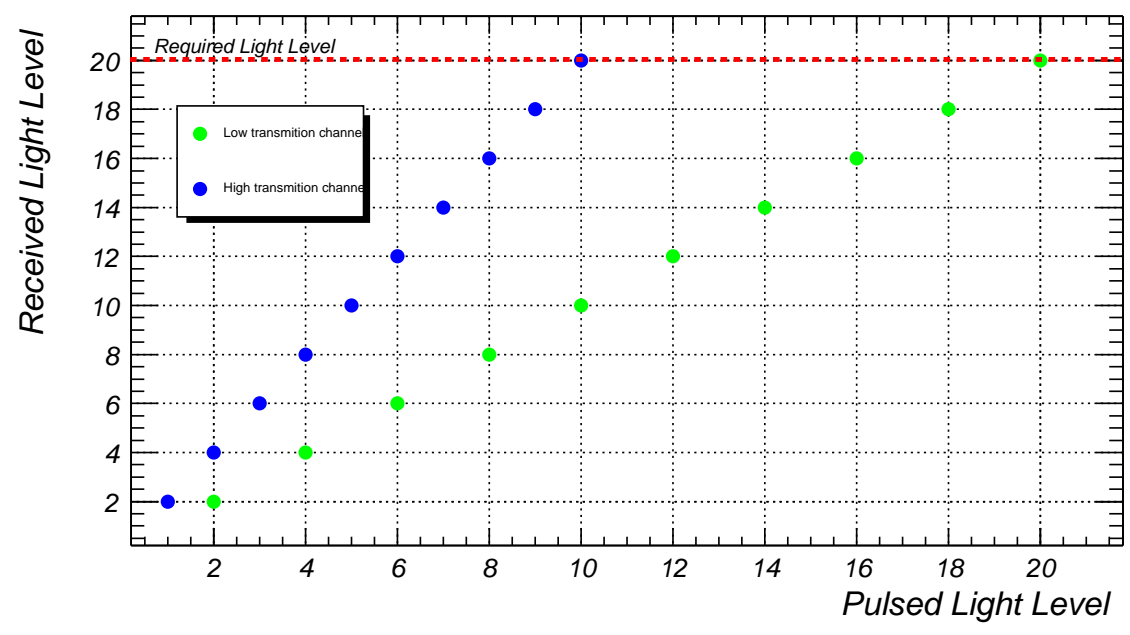

Figure 6.3: A demonstration of the need for uniformity in the light injection system. Each channel in the light injection system needs to be injected with enough light to map the response of the PMTs. As shown by this diagram, if two channels from the same LED differ greatly in their transmission, then the led will need to be pulsed at a higher brightness to compensate for the low-transmission channel. To achieve the same granularity in pulse heights, extra height settings are also needed

allowing quick and repeatable changes of amplitude. Finally, the wavelengths of light produced by blue LEDs can propagate down polymer fibre, greatly reducing the cost of the fibre plant as compared to the quartz fibre needed for UV laser light.

The LEDs chosen at the design stage were Nichia Chemical Industries [64]. However, due to changes in the specifications of these LEDs the response time changed considerably. This resulted in using Hewlett Packard HLMP-CB15 [65] LEDs as an alternative.

LEDs are very long lasting. In lifetime tests, LEDs were driven at high rates such that the integrated number of pulses would correspond to 2,000 years of MINOS running time. No LED has ever failed this test, or ever has reached the limit 
of its lifetime.

Figure 6.4 shows a comparison between the spectrum of light produced by the pulser system, and light from a laser-simulated scintillator. It is obvious that these are very different. However, figure 6.5 shows the spectrum of the two signals after being absorbed and re-emitted by the wavelength shifting signal fibre. Here it can be seen that the two spectra are very similar. This greatly reduces concerns about the wavelength dependence of the photocathode of the PMTs.

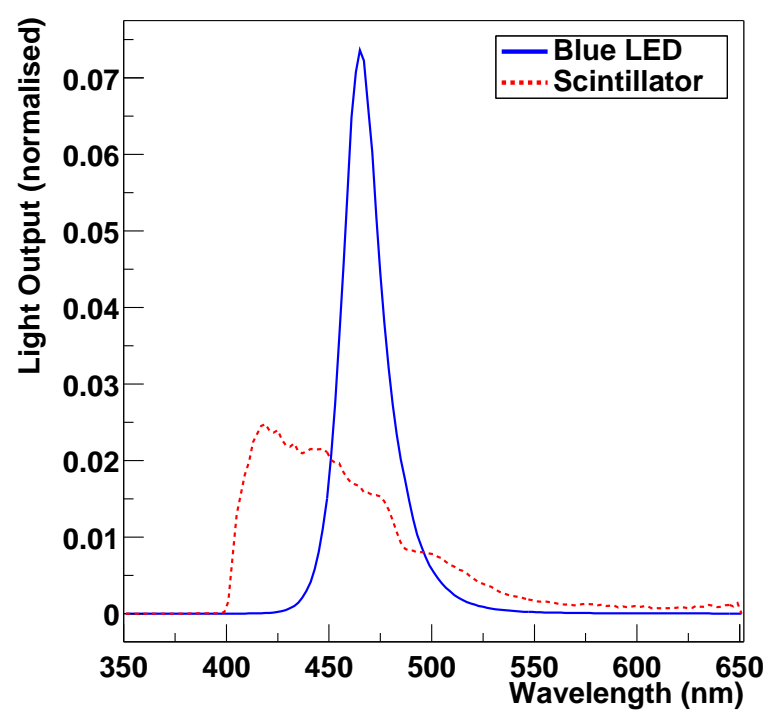

Figure 6.4: A comparison of the wavelengths of light emitted by the blue LED and laser-stimulated MINOS scintillator.

\subsection{Pulser Boxes}

\subsubsection{Mechanical Construction}

The pulser boxes are 21 " high crates that fit into a standard 19" rack. The crate is split into two compartments as seen in figure 6.6.

The smaller front compartment contains the electronics. There are three circuit 


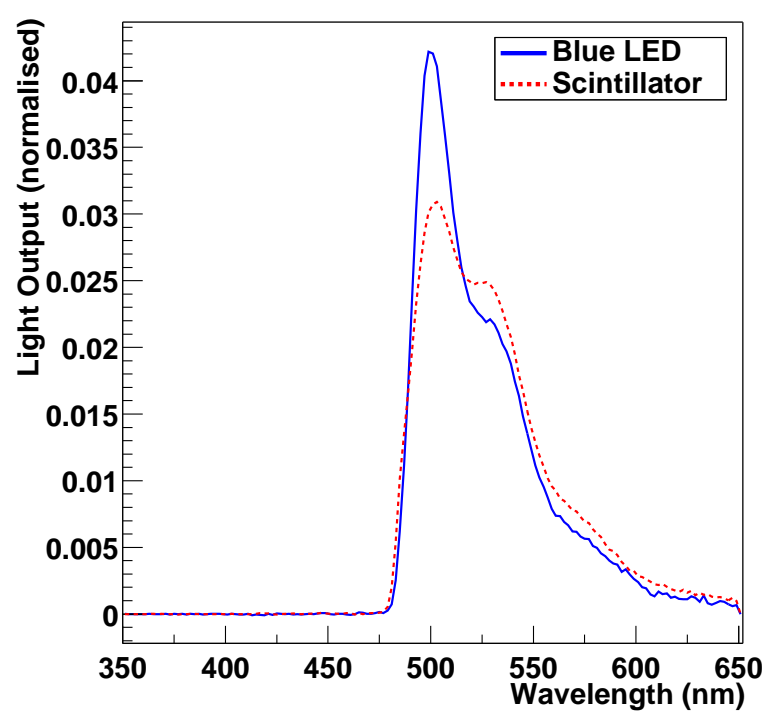

Figure 6.5: Spectrum of light emitted from wavelength shifting fibre when excited with the light in Figure 6.4.

boards in a standard pulser box; two driver boards that hold 10 LEDs each, and a control board. There is also a power supply and an RS485 interface.

The rear compartment contains the LEDs, distribution cones, ponytails and connectors. The cones distribute the light through the ponytails into the rear connectors. There are also three extra multi-way connectors on the rear of the box. Two of these feed light to the monitoring PIN photo diodes, and the third is spare. A single fibre connector supplies light to a dedicated PMT, the trigger PMT, that is fed a signal (of constant amplitude) at each pulse of an LED. The signal for the trigger PMT comes from an LED mounted on the controller card. This trigger PMT (tPMT) is read out in the normal data chain, and allows light injection events to be flagged as such.

\subsubsection{Cones and Collars}

In the light-tight rear section of the pulser box the cone and collar assembly fans out the light, from the twenty LEDs, to 67 connectors (64 normal, two for PIN photo 


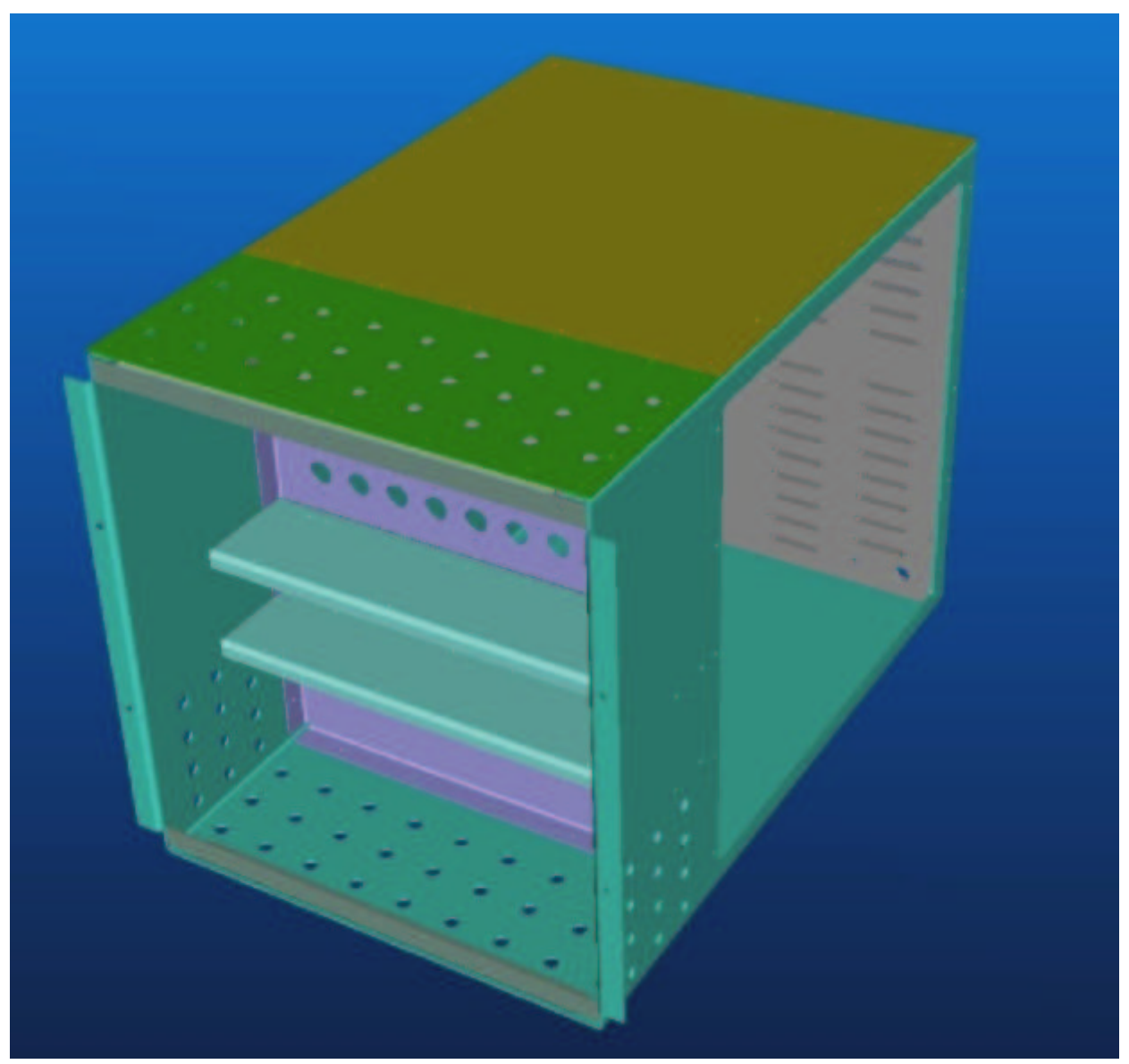

Figure 6.6: This is a front view of a pulserbox crate. The two shelves can be seen in the front compartment to hold the electronic pulser circuitry. The holes for the LEDs to be presented into the rear compartment can also be seen just above the shelves. On the rear of the pulserbox the connector positions have been drawn. 


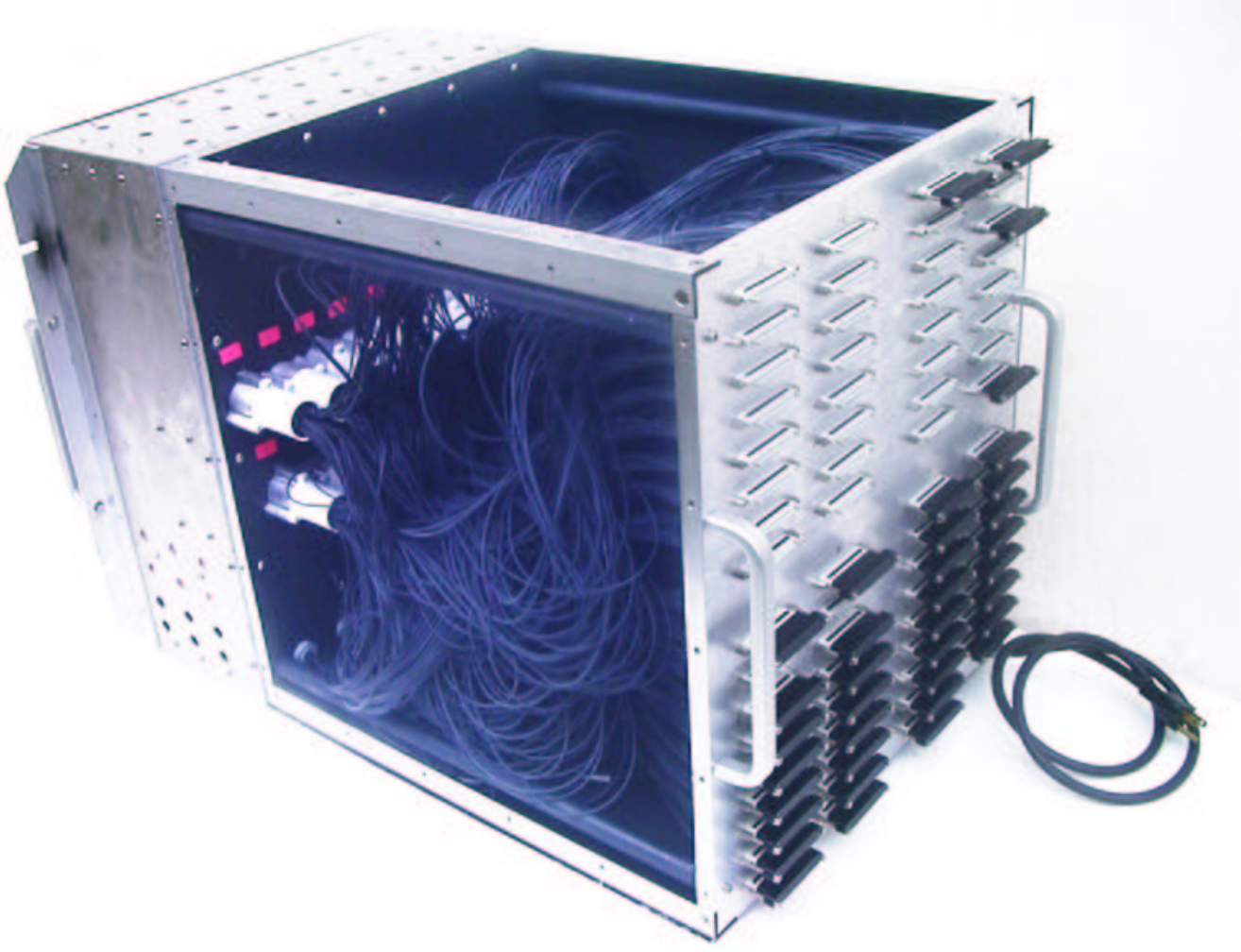

Figure 6.7: Photograph of a pulser box. The sides have been removed for clarity. The ponytail collars attached to the cones, and the bundles of fibre emanating from them are clearly visible. The complexity of the fibre "knitting" is also clear. 
diodes, and one spare). At this point several measures have been taken to improve the performance of the light injection system.

The light from the LED is reflected in a forward beam by an aluminium cone. To maximise light output into the fibres, highly polished mylar is cut to fit the interior of the cone. This cone reflects the light from the LED into a uniform as possible forward beam.

Figure 6.8 shows the pattern of light produced from this cone assembly. At this point there is only a $\sim \% 10$ variation in the light level over the surface of the fibres.

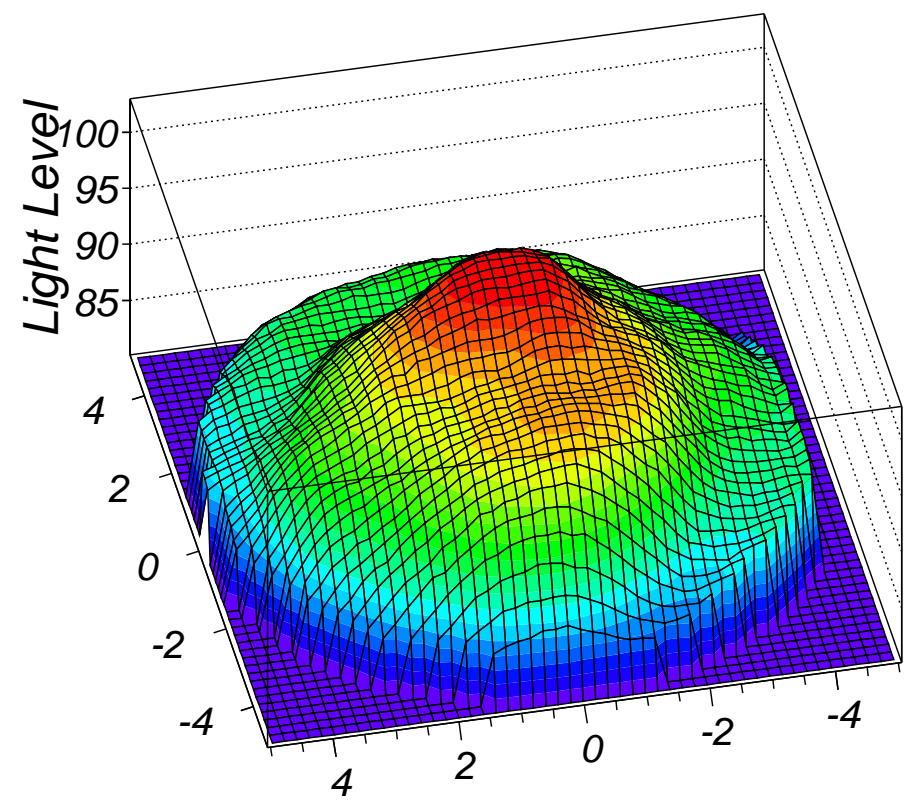

Figure 6.8: Plot showing the beam profile of an LED used in the pulser system.

Facing this beam is then the start of the ponytail and collar assembly. The ponytail is so called because 70 fibres are bundled together into a collar. The fibres are grouped into a ring so they avoid the central intense peak visible in figure 6.8. Each fibre is then slotted into a hole on a face plate of the collar, known as a tea-strainer, 
which is presented to the front of the cone. This tea-strainer is shown in Figure 6.9. Figure 6.10 shows the resultant uniformity of the light levels from these 70 fibres.

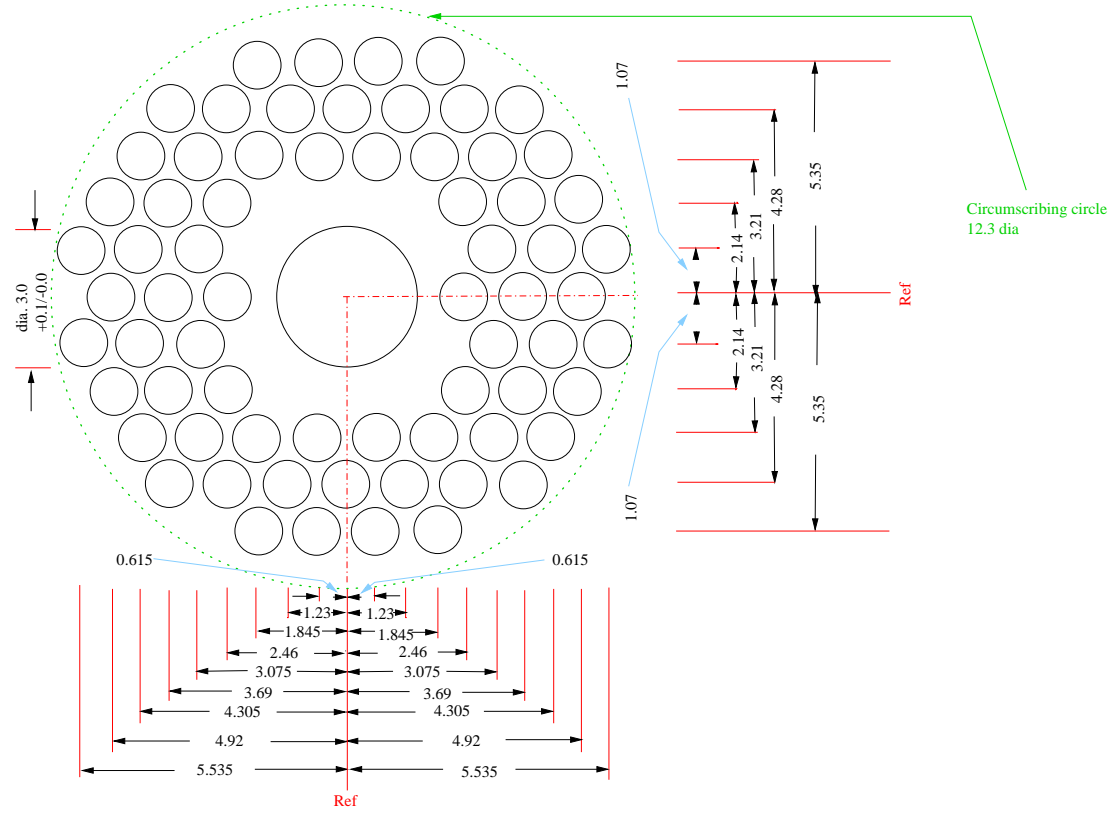

Figure 6.9: Diagram showing the tea-strainer plate. There is a hole for each fibre in the ponytail. The snout for the three elongated PIN diode fibres fits in the central hole on the tea-strainer.

In the centre of the tea-strainer face there are three fibres protruding from the face within a snout. These are the fibres that lead to the PIN photo diodes. These fibres are held closer to the LED and within the central intensity peak to maximise the intensity of the light delivered. This is necessary as PIN photo diodes are much less sensitive the PMTs. Figure 6.11 shows a photograph of a collar and cone, while figure 6.12 shows a schematic of the whole cone-collar-ponytail assembly.

\subsection{Light Injection Modules}

Each fibre from each pulser box leads to a Light Injection Module (LIM), these are moulded in manifolds on the end of each scintillator module. There are different 

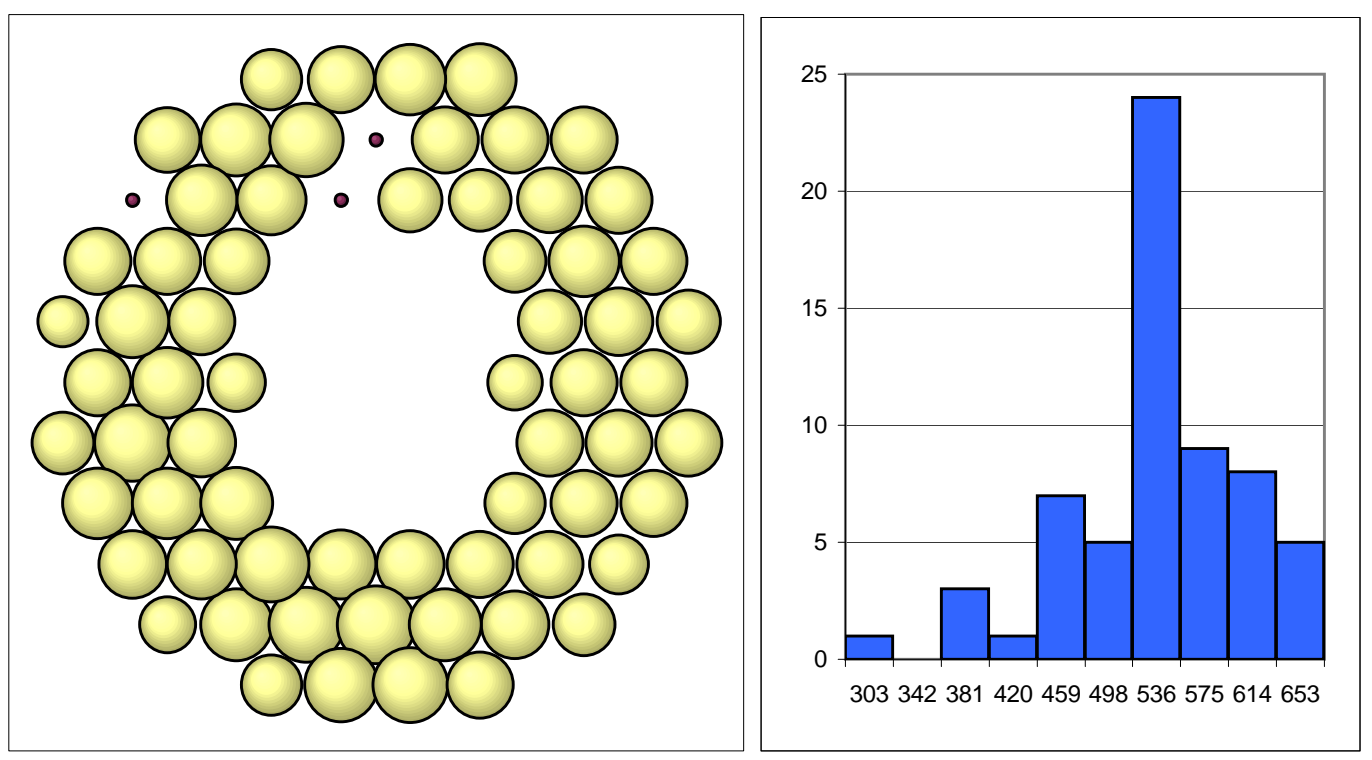

Figure 6.10: Uniformity of illumination of a fibre tea-strainer with an LED. In the diagram on the left, the area of each circle is proportional to the intensity of illumination. (The bundle included three spare fibres whose outputs were not measured). To the right is a histogram showing the distribution of intensities.

numbers of LIMs on each scintillator module, depending upon the number of fibres. For a 28-strip wide scintillator module there are 3 LIMs, two with 10 fibres, and one with only 8 . When there are only 20 strips in a scintillator module there are only two LIMs, with 10 fibres in each.

A module can be seen in figure 6.13, with the green signal fibres leading through LIMs. The reflective part of the LIM fits over these modules. Figure 6.14 shows the design of the LIMs, and how they are mounted onto the modules.

The light injection modules are design to distribute the light from the pulser fibres into the green WLS signal fibres. Figure 6.15 shows a schematic of this.

Investigations were made into the best design for maximising the light absorbed 


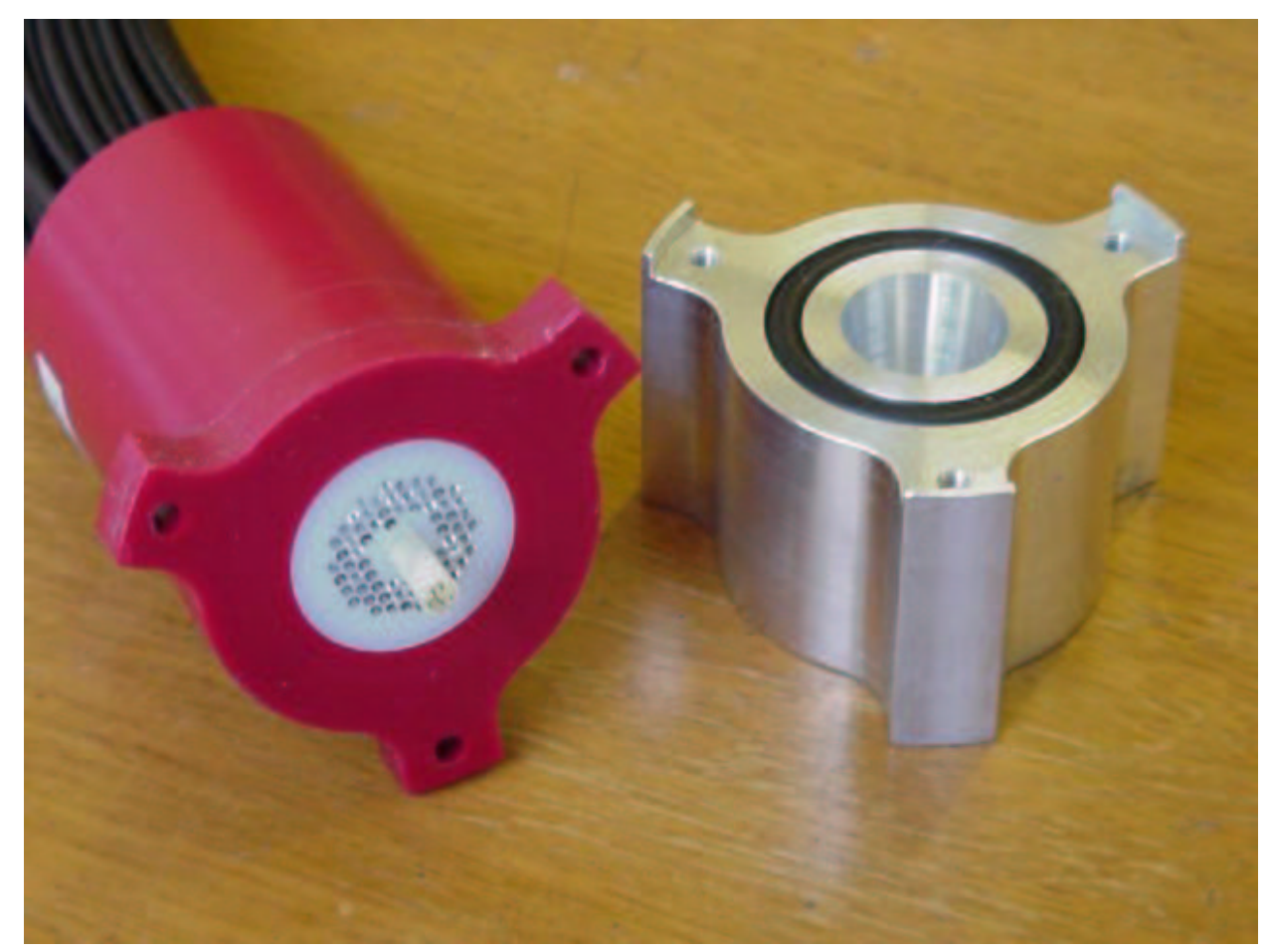

Figure 6.11: Photograph of a cone and collar.

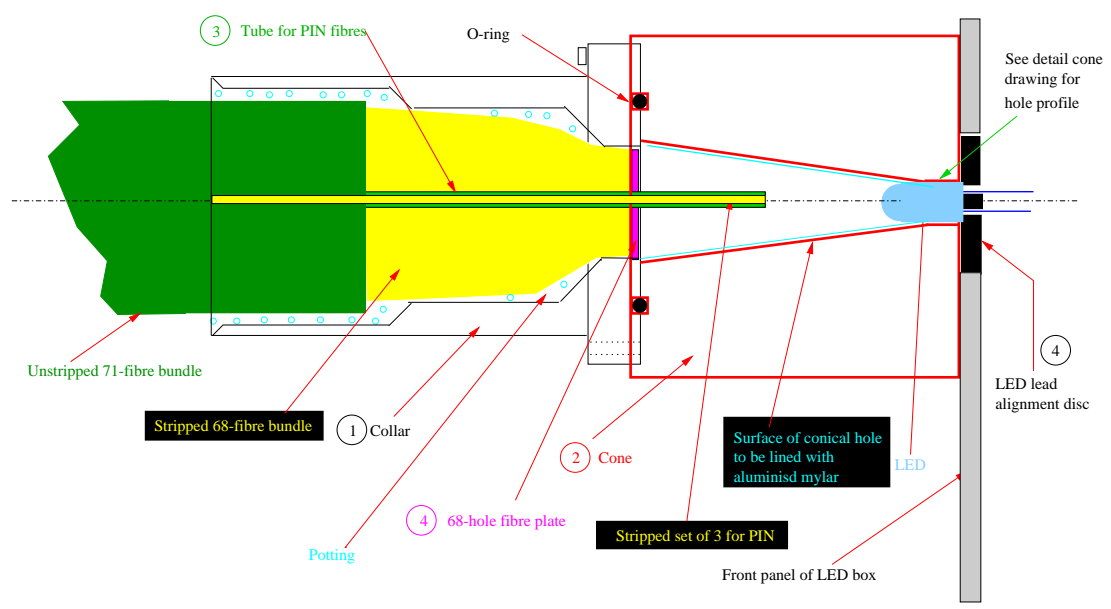

Figure 6.12: Schematic showing the cone and collar assembly. Notice the elongated snout protruding into the cone for the PIN diode LEDs. 


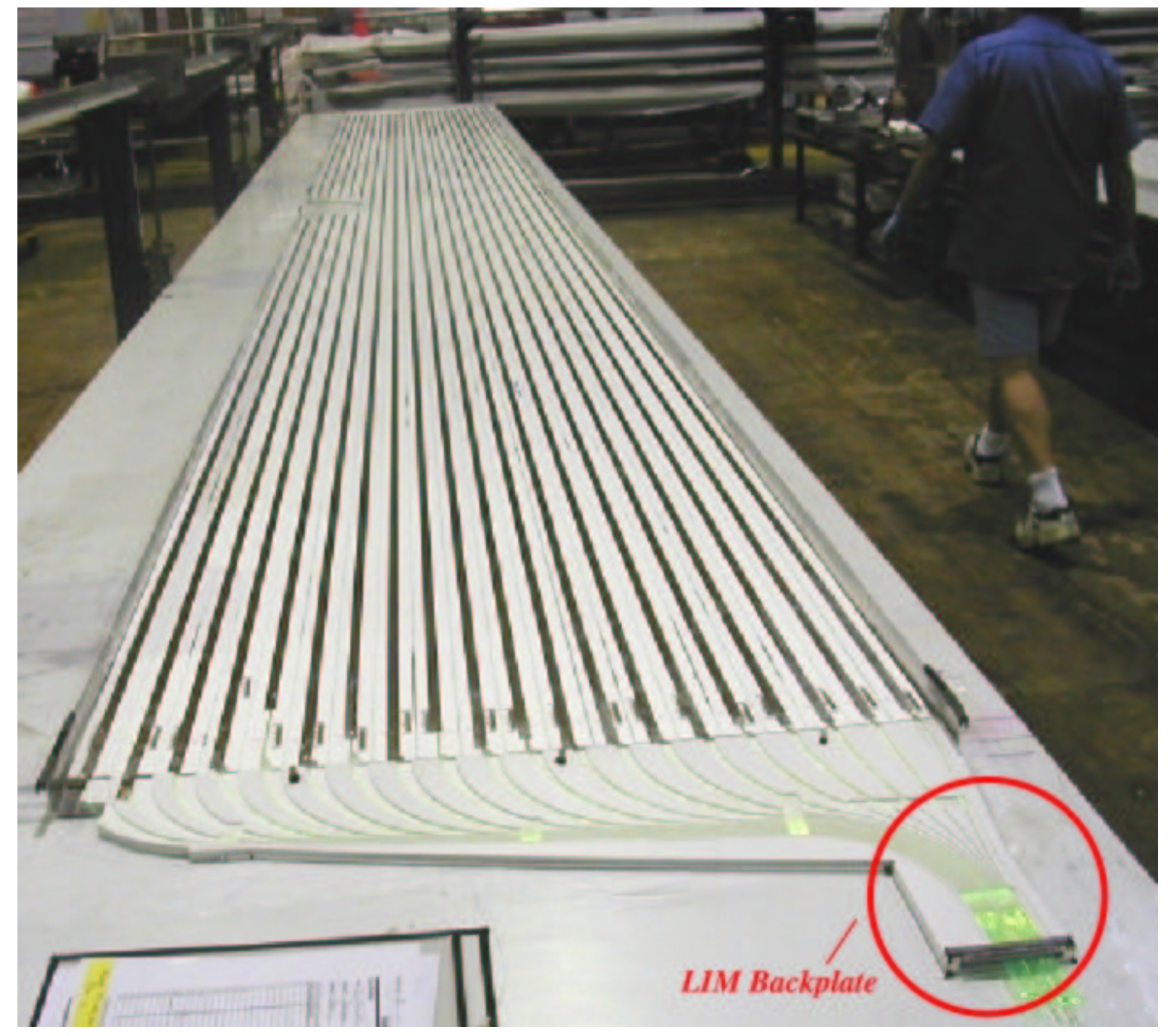

Figure 6.13: Photograph of a scintillator module during construction. This is a twenty strip wide module. At the front of the module one can see the green WLS fibres leading out of a grey connector; after construction these fibres will be trimmed and polished. Just behind the grey connector is the section that the LIM fits over, as seen in figure 6.14 . 


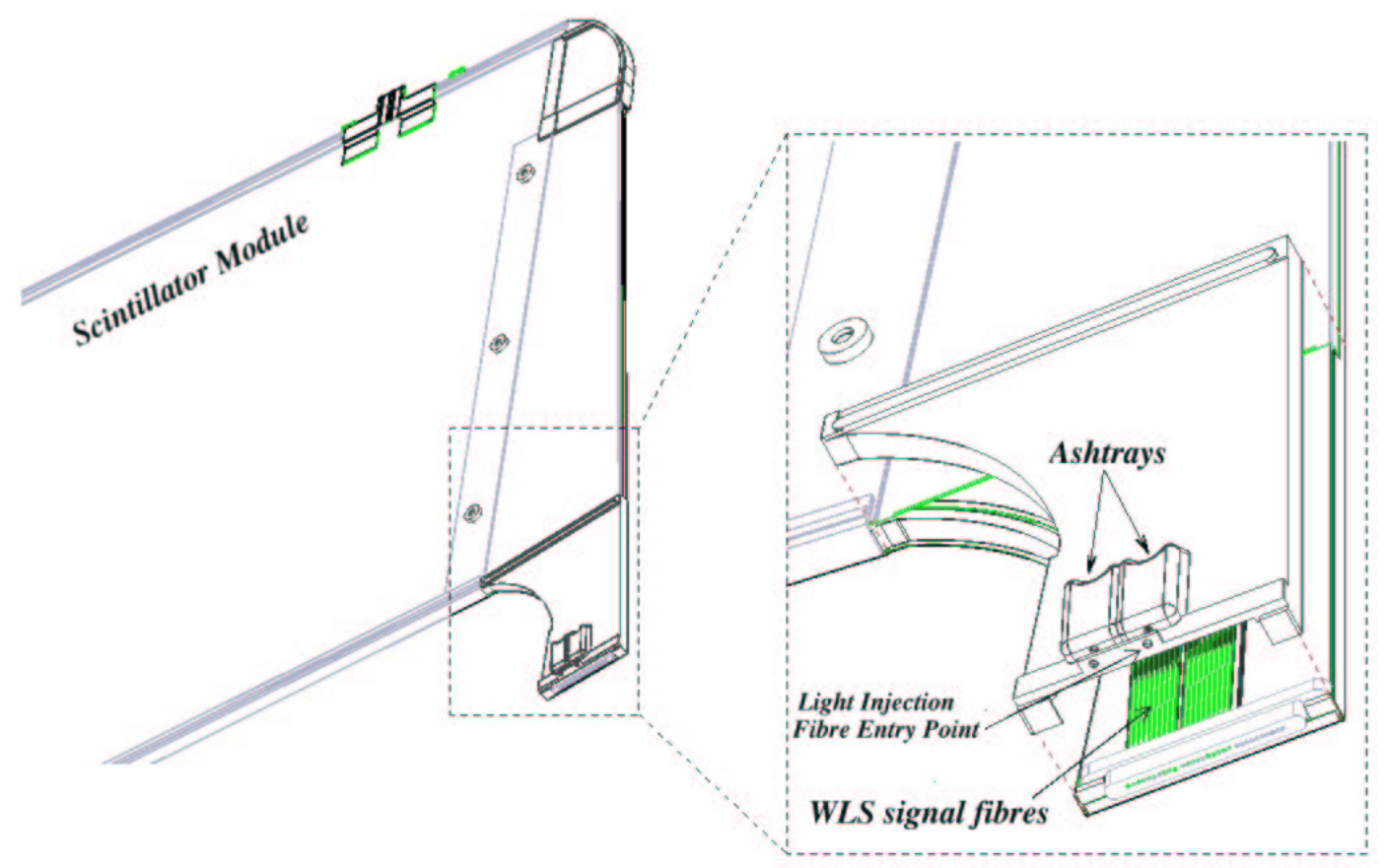

Figure 6.14: Schematic of a Light Injection Module. This manifold only has 20 signal fibres, therefore it has two ashtrays of 10 fibres each.

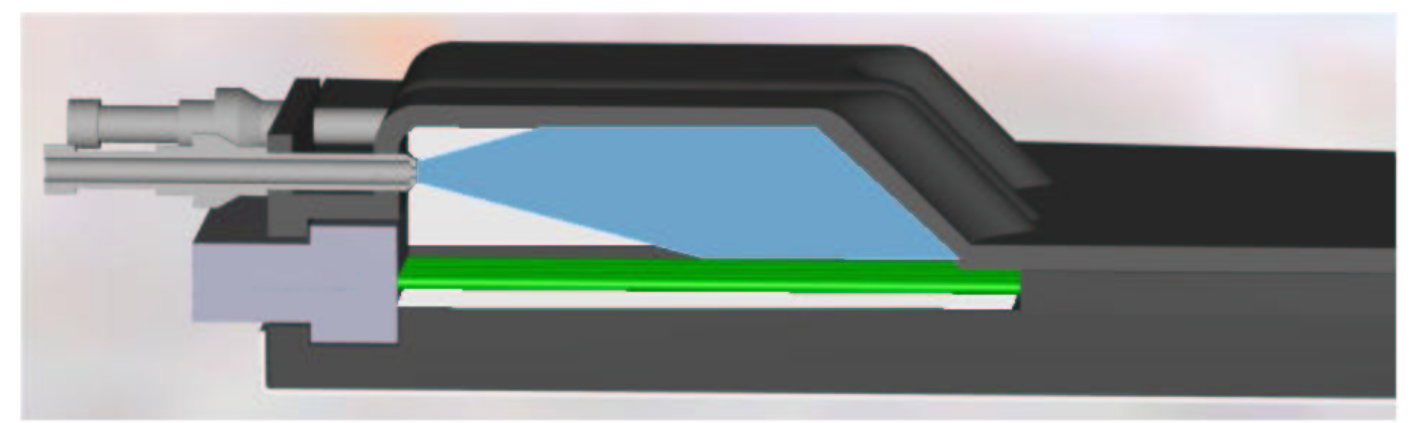

Figure 6.15: LIM cutaway profile. This figure shows a cut away profile of the LIM. The light injection fibre connector can be seen shinning light into the module, onto the green signal fibres. 
by the signal fibres within the LIM. Figure 6.17 shows the results of two different LIM back designs. The first was a simple $45^{\circ}$ slope; the second was a circular back, tilted to $45^{\circ}$, as shown in figure 6.16 . The circular sloping back gives better uniformity along the LIM, however this is at a slight sacrifice of the brightest amplitude. Unfortunately due to time restraints there was not time to optimise this design, however the final construction version (seen in figure 6.16) showed only $10 \%$ variation in light levels absorbed by the green signal fibres.

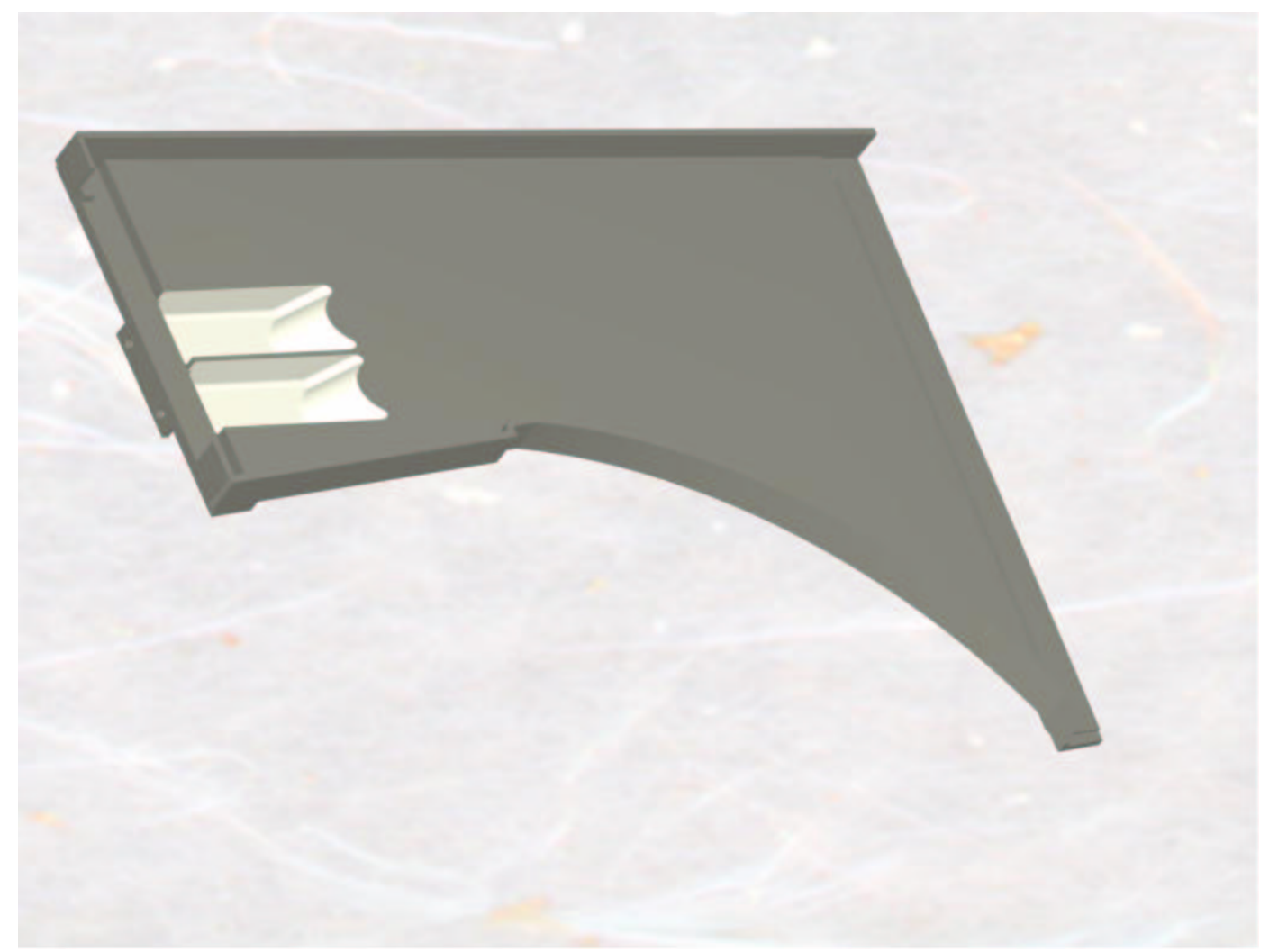

Figure 6.16: The LIM cover. This portion of the LIM fits over the manifold as show in figure 6.14. The reflective portions can be seen as white, representing the reflecting paint. Note the semi-circular curved shape of the back wall of the LIM.

Both paint and reflective film were tested to increase the internal reflection of the LIMs. The paint used was $\mathrm{TiO}_{2}$ reflective paint (Bicron BC625). The Mylar was extremely difficult to cut and to fit in a LIM without scratching and damaging the 
reflective surface. As can be seen from the plot (Figure 6.18) the paint was the most reflective. Paint was therefore used in the final design to cover the black plastic LIMS. On the underside of the LIM, under the signal fibres, reflective plastic strips were placed to provide further reflectivity.
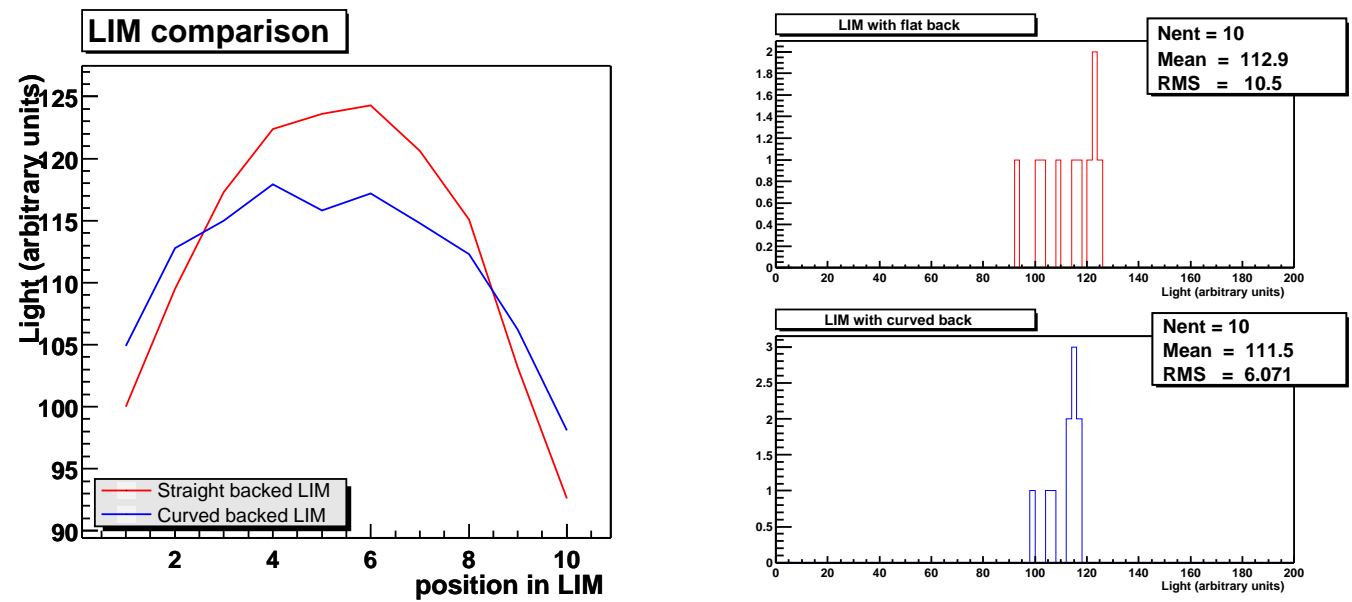

Figure 6.17: Results of testing of two LIM shapes. The red plot shows the results for a LIM with a straight reflector on the back panel. The blue is for a curved panel. The curved panel lifts the light levels of the edge fibres within the LIM, producing a more uniform light distribution to the fibres, albeit at a sacrifice of maximum brightness for the central fibres.

\subsection{PIN Diode Linearity and Stability}

\section{Linearity}

One of the design constraints placed on the light injection system was that it was to provide a measure of the absolute linearity of the light levels pulsed. It was decided that PIN diodes would be utilised for this task. The PINs used are Hamamatsu S5971, which have a number of desirable features including a relatively large surface area to maximise light intake from a $1 \mathrm{~mm}$ fibre. They also have a fast response time. 


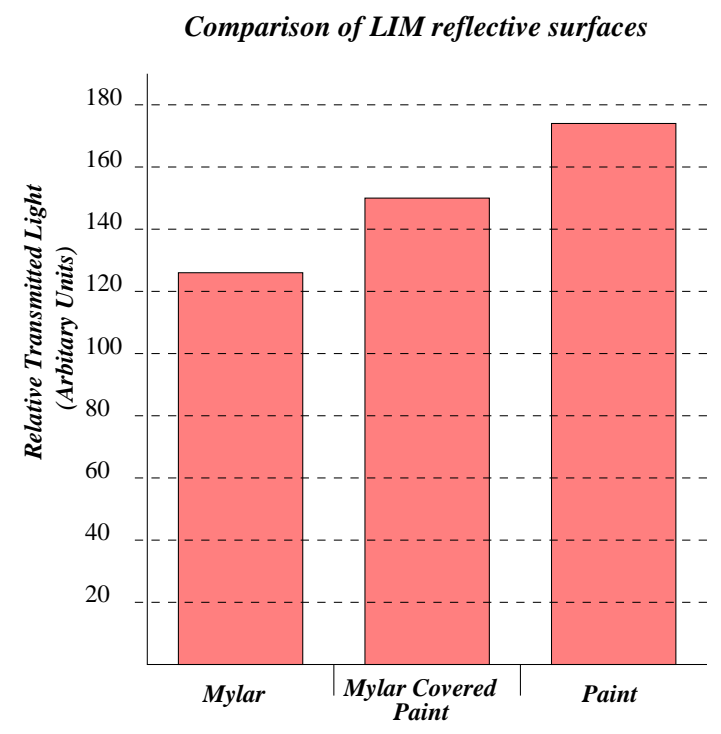

Figure 6.18: Comparison of reflective surfaces of the LIM.

\begin{tabular}{|c|c|}
\hline Active area size & $1.2 \mathrm{~mm}$ diameter \\
Spectral response & $320 \mathrm{~nm}$ to $1020 \mathrm{~nm}$ \\
Dark current & $0.07 \mathrm{nA}$ typ. \\
Temperature coefficient of dark current & $15 \%{ }^{\circ} \mathrm{C}$ \\
Photo sensitivity @ 470nm & $0.24 \mathrm{~A} / \mathrm{W}$ \\
Temperature coefficient of photo sensitivity & $0.01 \%{ }^{\circ} \mathrm{C}$ at lab temperatures \\
\hline
\end{tabular}

Table 6.1: PIN diode specifications

A high/low gain test was used to test the linearity of the PIN diodes. The PIN was pulsed with a range of light levels, and at each light level a reading was taken with the light directly incident on the PIN diode. A reading was also taking with the light passing through a $10 \%$ transmission neutral-density filter. The results from this test are shown in Figure 6.19. It can clearly be seen that there was no non-linearity present. 

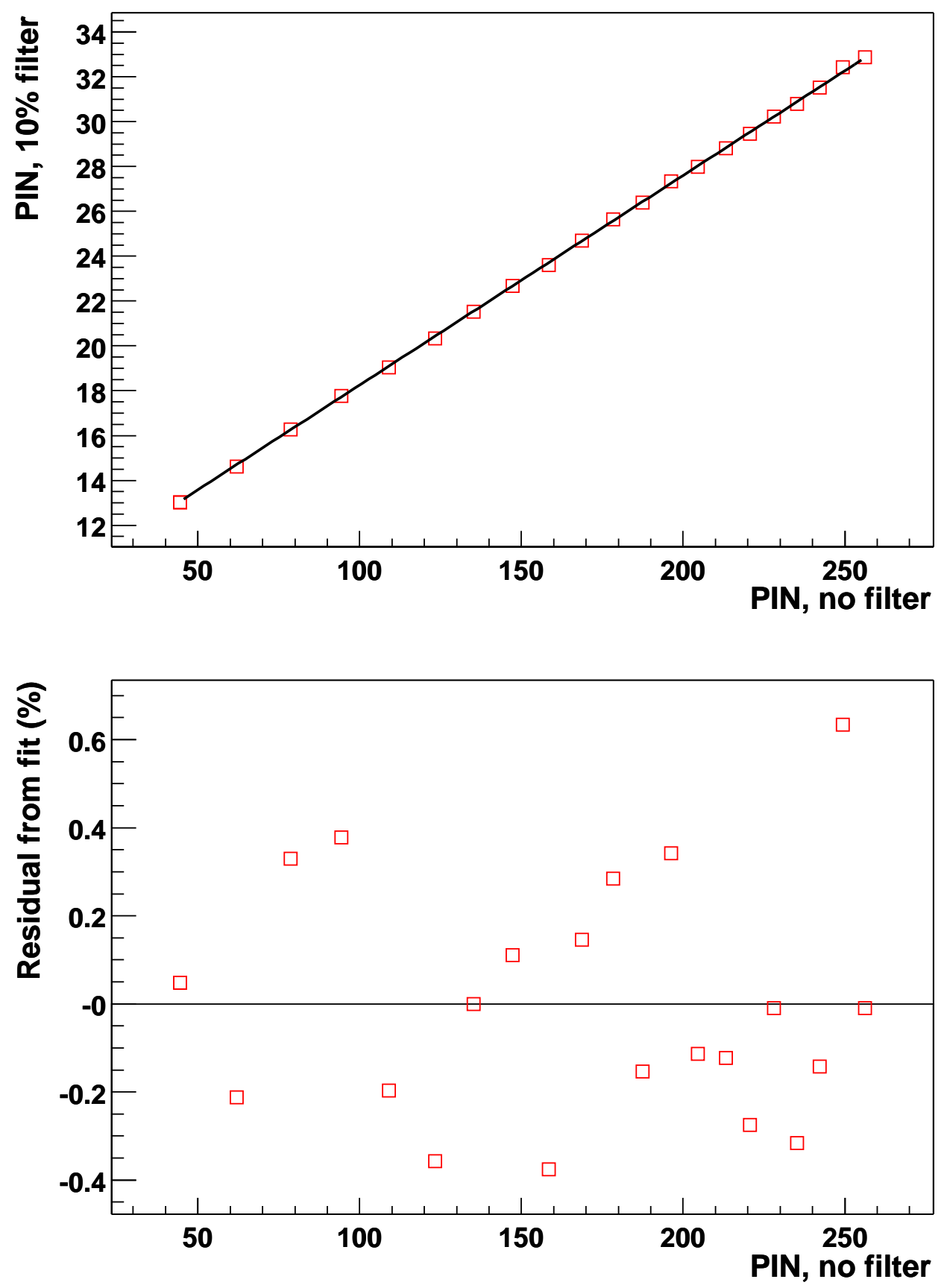

Figure 6.19: Results from the linearity tests of the PIN diodes. The residuals from a straight line fit are clearly purely statistical, showing that there is no non-linearity of the PIN diode over the whole range of light levels. 


\section{Stability}

The PIN diodes were also tested for stability. A test stand was set up with two separate PIN diode circuits illuminated by the same pulsing LED. These PIN diodes were read out by a digital oscilloscope. Figure 6.20 shows the results from this test. It can be seen that there was a change in the light level emitted from the LED at 15 days; this jump was followed by both PIN diodes. The ratio of one PIN signal to the other was not affected above the $1 \%$ level. At the 40-day mark the LED light level changed again, and once again the PIN doides stayed perfectly in line with each other. This shows that the PIN diodes and amplification circuitry are stable to within $1 \%$ over a one-month timescale, as required.

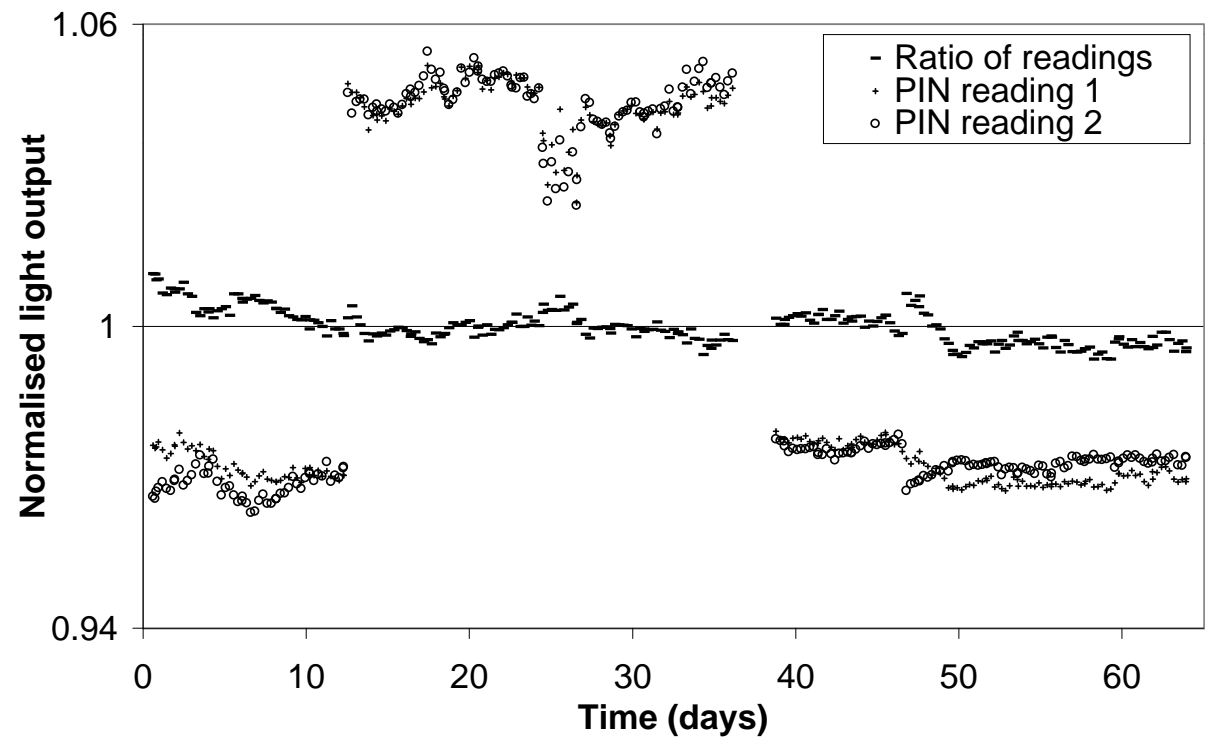

Figure 6.20: Results from the PIN stability test. The shifts in light levels at around ten and forty days were due to accidental physical movement of the pulsing fibre positions. However, these anomalies were successfully tracked by both PIN diodes. The ratio of both never wandered by more than $1 \%$. 


\subsection{Trigger PMT}

From time to time with the MINOS detectors, we will want to take light injection runs with light levels at a fraction of a photo-electron, so as to be able to measure the single photo-electron spectrum. This implies that a significant number of pulses will result in no readout from the PMT. A trigger is therefore needed to prompt the DAQ to read out. To do this there is a so-called trigger PMT. The trigger PMT is fitted in a modified MUX box, which only has one PMT. It is read out in an identical way to the other boxes. The DAQ software can be configured to store all events that are in the same time slot as the trigger PMT pulses, allowing the zero readings to be counted. Each of the 16 far detector pulser boxes has a single fibre that carries light to this PMT.

\subsection{Electronics}

The pulser box is controlled by a PIC16F877 microprocessor. Communication with this processor is via an ASCII RS485 link with a standard PC running Linux. All parameters of the pulser box characteristics can be controlled via software. Table 6.2 shows some of the pulsing options and their parameters. The pulse height (i.e. voltage applied to the LED) is controlled by an applied voltage from a 10 bit ADC, providing 1024 settings. The pulse width is controlled by a programmable delay line. This can provide 7 different width settings from 15 to 36 ns. Some sample pulse shapes covering the whole range are shown by figures 6.22 and 6.21 . It should be noted that the sharp pulse shapes produced by the pulsing system are "smeared" by the absorption and re-emission of light within the the WLS fibre. Figure 6.23 shows the 50 ns tail that is added to the pulse shape when passed through the WLS fibre.

In normal operation, the controlling PC sets the pulser box parameters to carry out a number of pulses (normally 1000 for reasonable statistics) at a set height and 


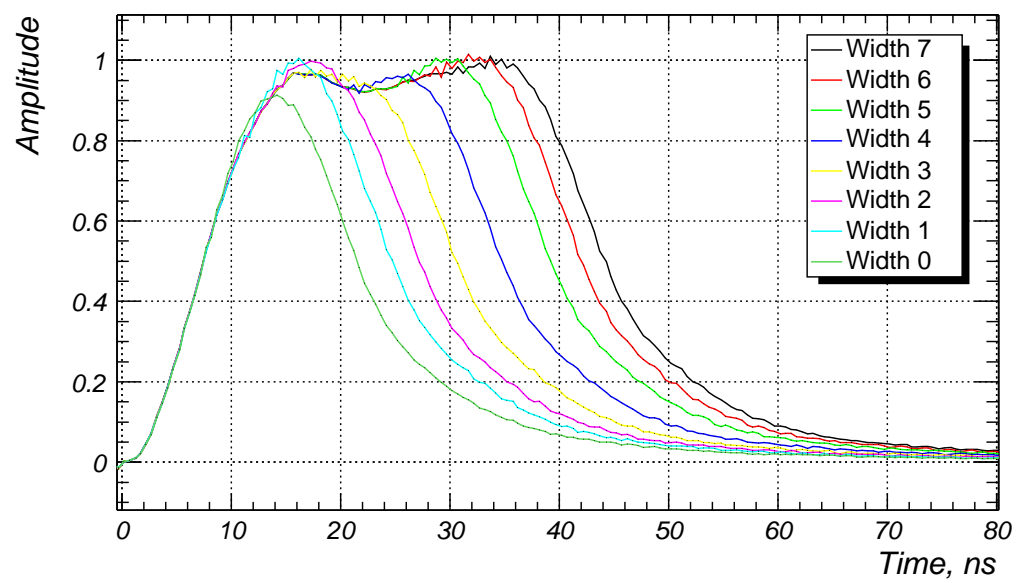

Figure 6.21: Pulser light output shapes. Eight original widths are possible, providing a pulse duration ranging from 15 to $36 \mathrm{~ns}$. Additional programmable widths are available to extend the pulse further if needed.

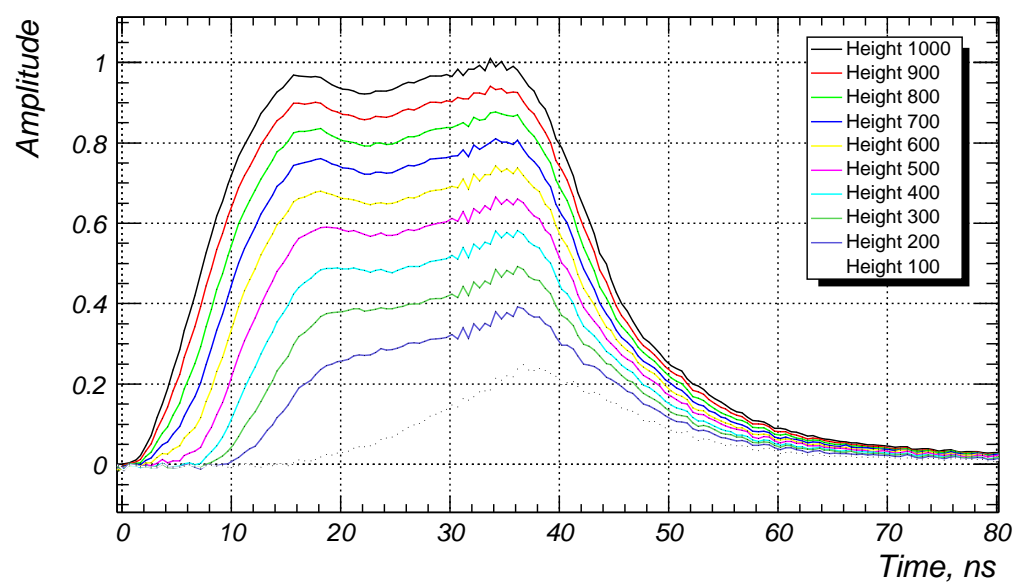

Figure 6.22: Pulser light output shapes for different pulse height settings. As well as the eight pulse width settings there are also 1024 height settings, from 0-1023. 


\begin{tabular}{|c|c|}
\hline LED number & $0-20$ \\
Pulse height & $0-1023$ \\
Pulse width & $0-7(15)$ \\
Pulse repetition rate & $1 \mathrm{~Hz}-65 \mathrm{kHz}$ \\
Number of pulses & $1-65000$ \\
\hline
\end{tabular}

Table 6.2: Pulser parameters. All can be set before a pulser run via computer control.

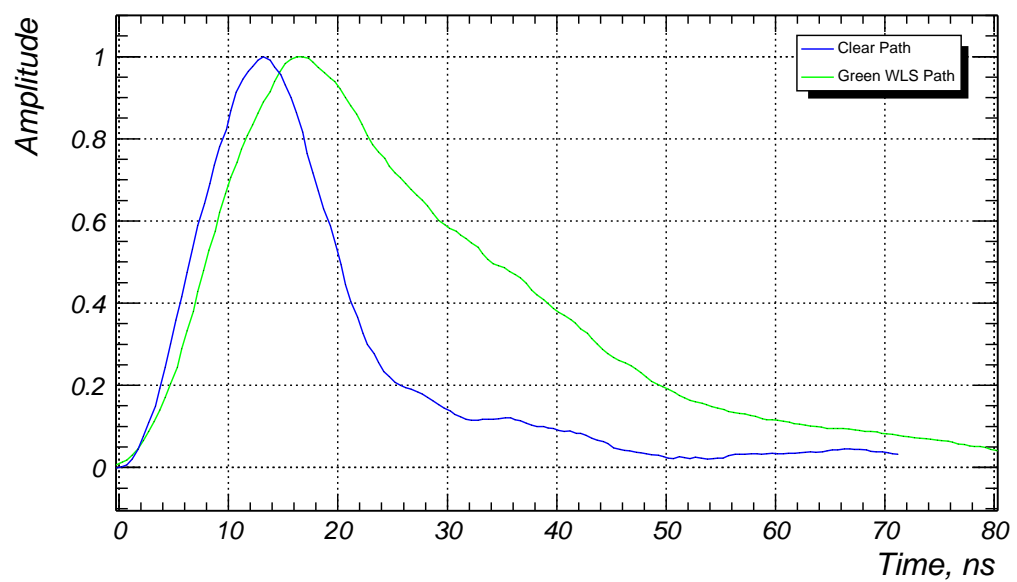

Figure 6.23: The blue line shows the pulse profile for a width setting of 0 and a height of 1000. The green line shows the distortion of this pulse due to the absorption and re-emission of light within the WLS fibre. 


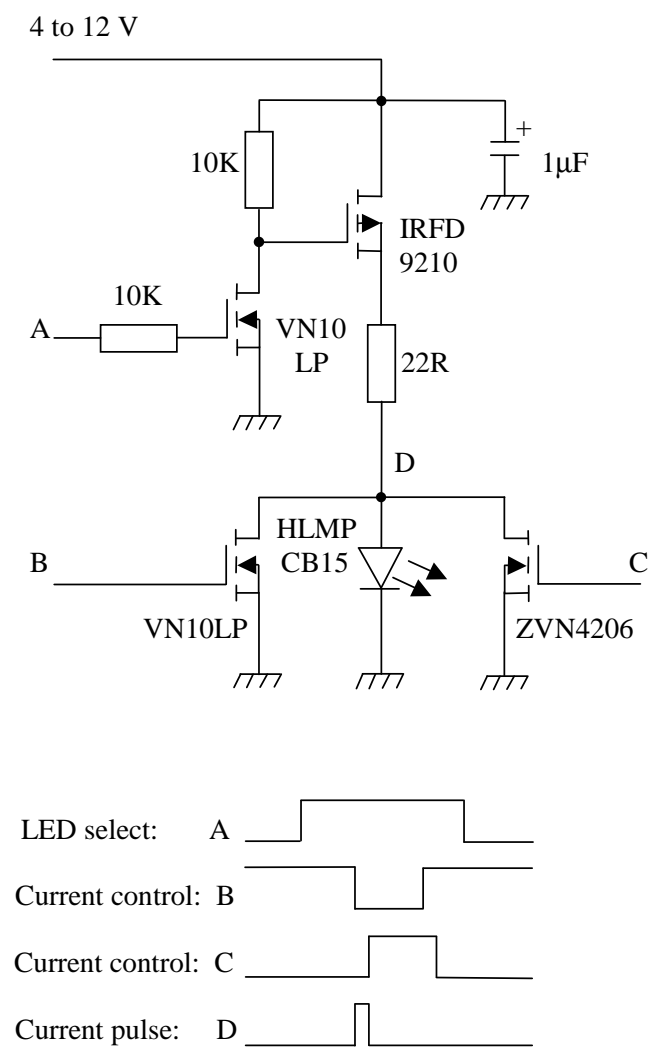

Figure 6.24: Schematic pulser electronic circuitry designed by Dick White from Sussex University. 
width. It is then up to the microprocessor in the pulser box to control the pulse sequences. The pulser boxes also have a veto connection, allowing the pulsing to be paused during beam cycles. Although the pulser boxes are capable of pulsing up to $30 \mathrm{kHz}$, the pulse rate during data taking is limited to $\sim 33 \mathrm{~Hz}$. As each pulse illuminates 1280 channels directly, causing very high data rates, the DAQ system can become saturated. As there are 16 far detector pulser boxes, each with 20 LEDs, a full drift point run, used to monitor changes in PMT gain, with one light level setting and 1000 pulses on all LEDs takes about 100 minutes. A full gain curve run, used to map the PMT gains, with 10 height settings per LED will take 1000 minutes $(\sim 13 \mathrm{hrs})$.

The light injection installation at the far detector is now complete. There are 16 pulser boxes installed, each with 20 LEDs and each LED supplying light to $\sim 1280$ fibres.

\subsection{Linearity and the UV LED}

During the testing of the light injection system non-linearity was observed while using blue LEDs. When the response of the PIN photodiodes to blue light was compared to the response of the PMTs to the green light emitted by the WLS fibre the non-linearity in figure 6.25 was seen.

In lab tests however, no difference was seen when comparing the response of two PMTs being illuminated by the same LED. The linearity of the PIN photodiodes had also been proved as shown above. Figure 6.26 shows the LED spectra for high and low current pulses for the blue light received by the PIN photodiodes, and the green light received by the PMTs.

The source of this difference becomes apparent when the pulsed spectra are viewed along with the absorption spectrum of the WLS fibre. This is shown in figure 6.27. This figure shows how the pulse spectra of the blue LED falls over a 


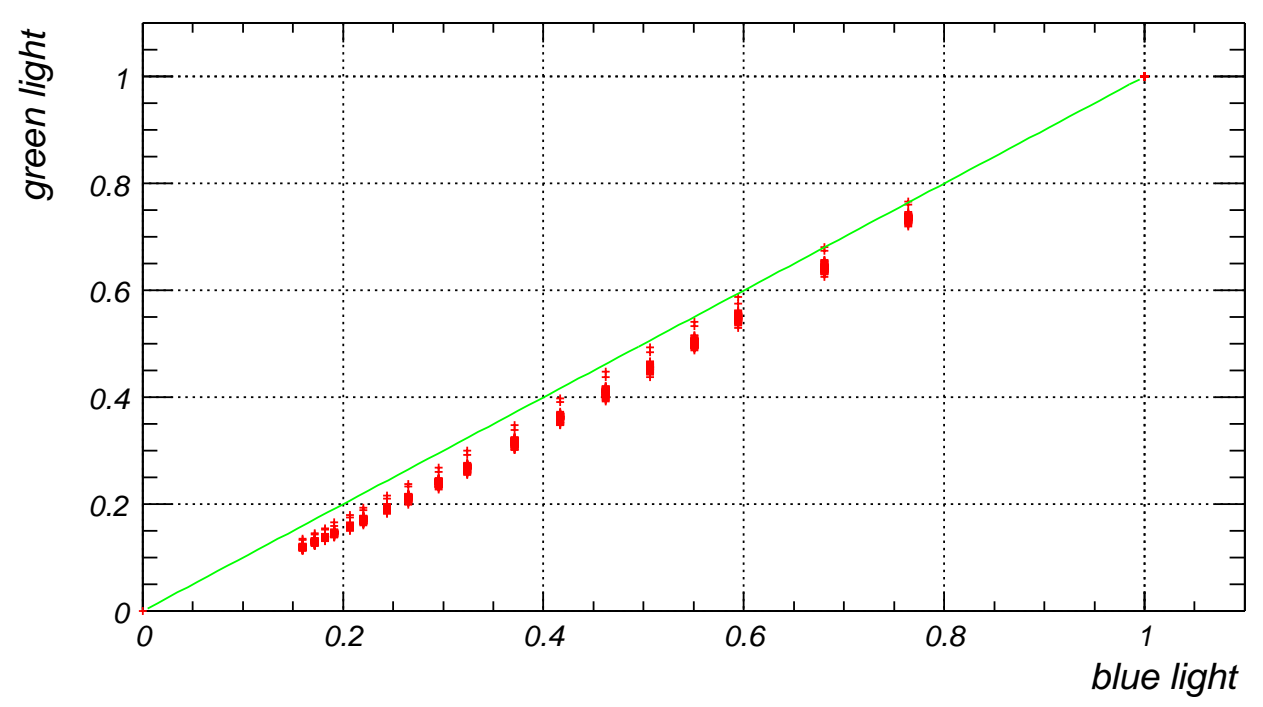

Figure 6.25: A plot showing the non-linearity, seen by the calibration detector, of the pulser system. Each point shows the response of a M16 PMT pixel to the green light versus the response of the PIN photodiode to the blue light. The solid lines shows true linearity. The data are normalised to the highest point. 


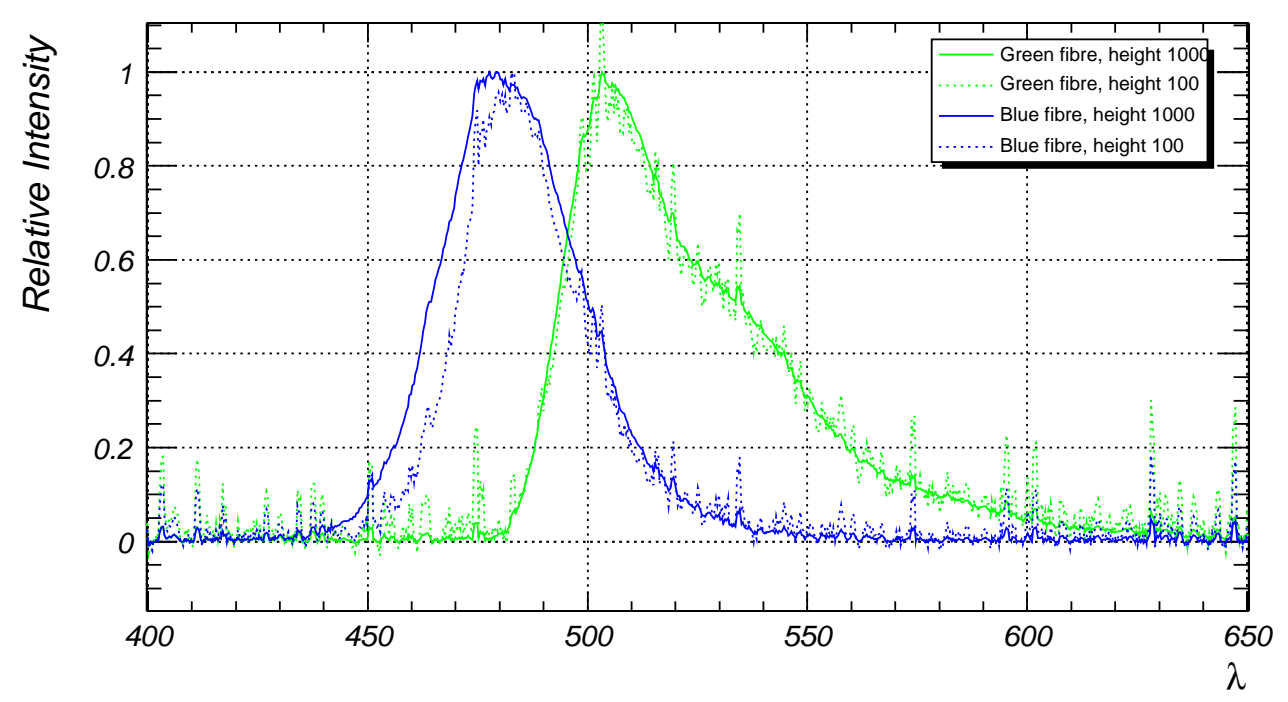

Figure 6.26: Wavelength spectra of the two LED pulses, one produced by the green WLS fibre, the other the raw blue LED spectra. They have been normalised to have the same amplitude 
very rapidly changing area of the WLS absorption curve. This causes the spectra changes between the high and low-current pulses to differ between the raw blue light from the LED, and the green light re-emitted by the WLS fibre.

To find a solution to this, the spectral properties of several types of UV LEDs $[64,66]$ were investigated, as shown in figure 6.27. The spectra from these UV LEDs changed less for different current settings, also they occupied an area of the WLS absorption curve where the response is much flatter. The result of this is that the UV LEDs provide a much more linear curve as proved by figures 6.28 and 6.29 .

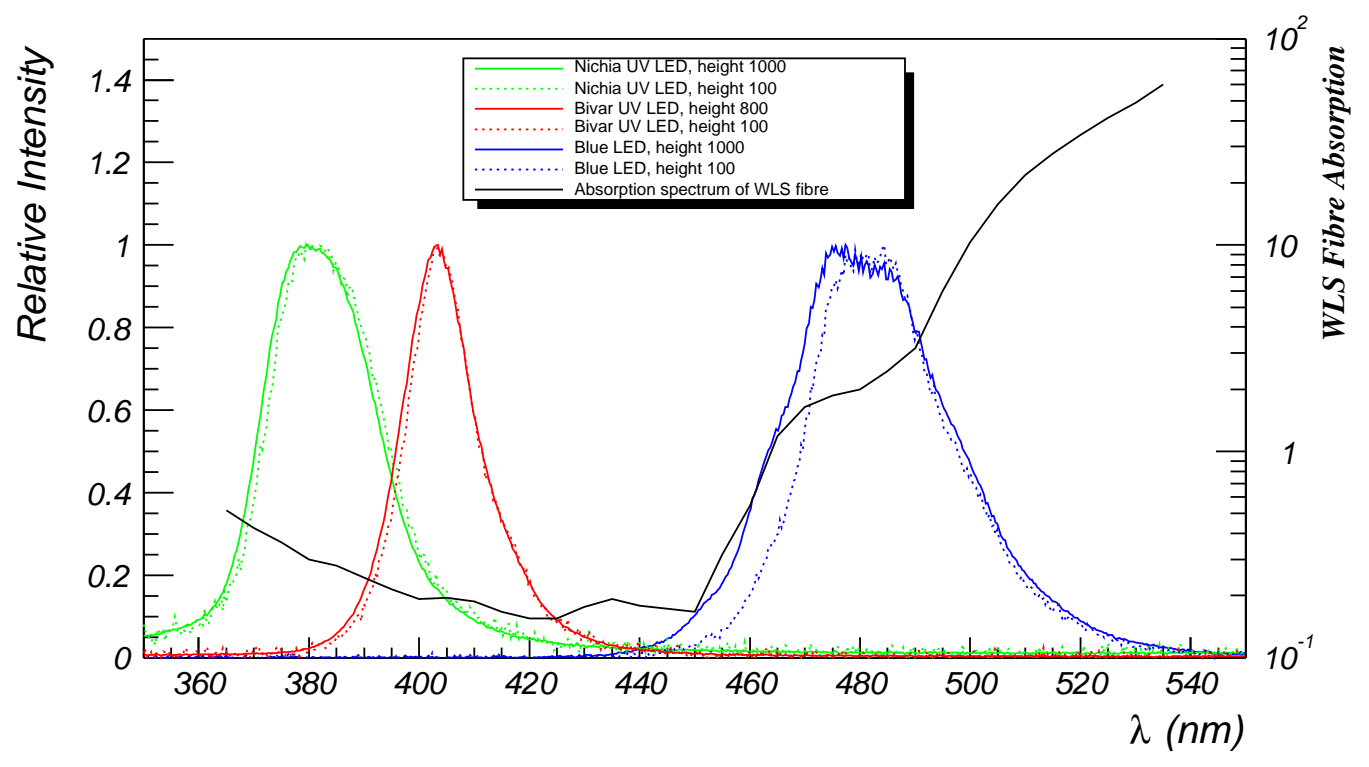

Figure 6.27: Here is shown the spectra for the LEDs along with the absorption curve for the WLS fibre [67]. Although there is still a slight difference in spectra's for the UV LEDs, the changes in the absorption of the WLS fibre are far less dramatic at this point.

As well as providing a pulse that allows the system to reach linearity, the Bivar UV LEDs are also brighter than the blue LEDs. They also achieve the same level of uniformity throughout the system. Figure 6.30 shows the pulse shape of the Bivar 


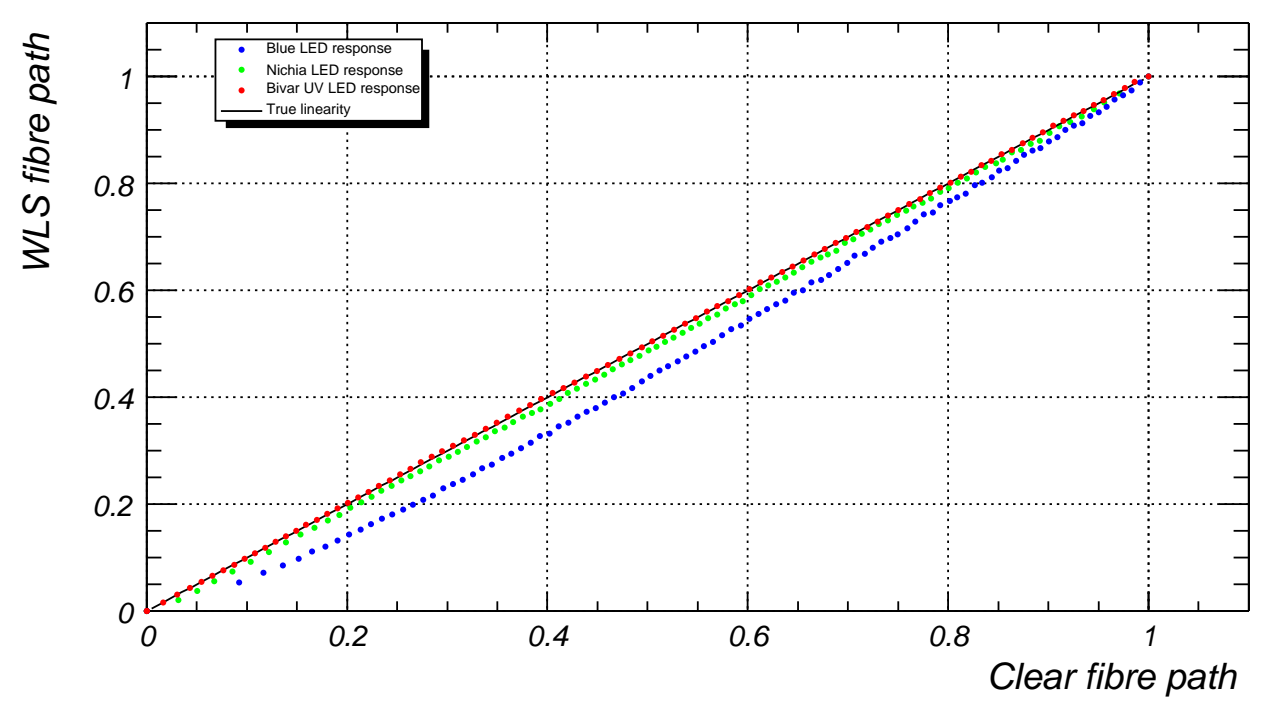

Figure 6.28: The final result. The Nichia UV LEDs still do not give us perfect true linearity, however the Bivar UV LEDs do indeed give us linearity, to within measurement error. This can be shown by the residuals in figure 6.29 . 


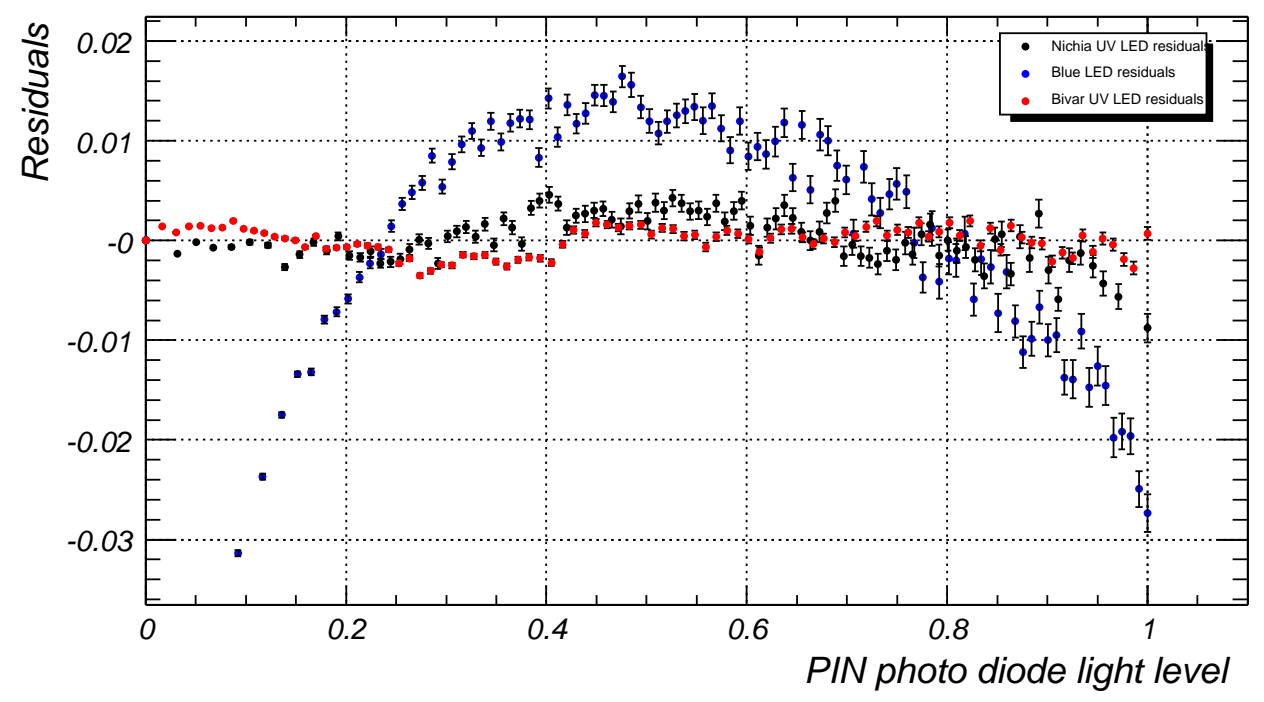

Figure 6.29: The residuals of the linearity curves from UV and Blue LEDs. The Bivar LEDs are clearly the most linear.

LEDs, it can be seen that the pulse width is also reduced allowing sharper pulses. 


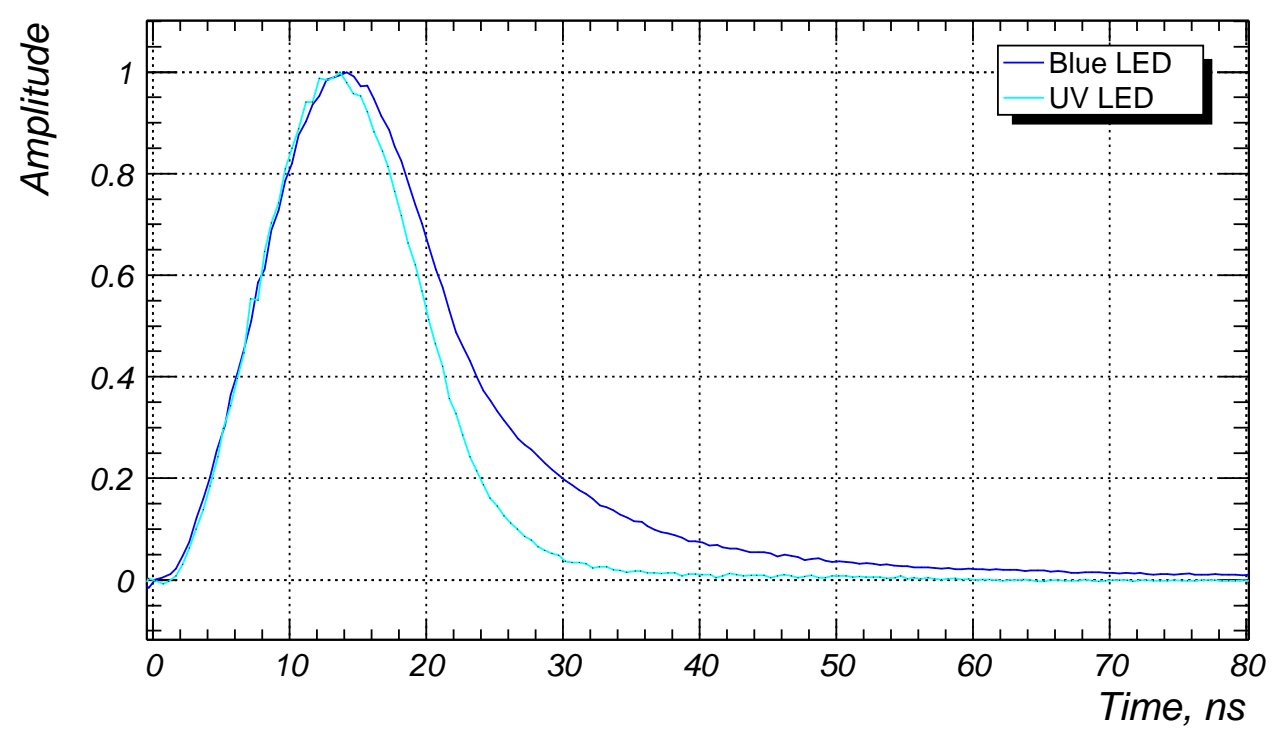

Figure 6.30: The improved pulse shape from Bivar UV LEDs. The rise time is the same, but the fall time is reduced. 


\section{Chapter 7}

\section{Hadronic Production and NA49 Introduction}

\subsection{Introduction}

There were two main motivations for carrying out a hadronic production investigation using the NA49 experiment:

\subsubsection{MINOS Neutrino Oscillation Parameters}

The first motivation was to be able to reduce the errors involved in the calculations of the neutrino oscillation parameters in MINOS. Several methods of calculating the neutrino oscillation parameters rely upon accurate modelling of the neutrino beam, to allow the fluxes to be calculated at each detector. Figure 7.1 shows the predictions by four of the main models for hadron production generation of neutrinos at the near detector. Figure 7.2 shows the ratio of this to the far detector neutrino fluxes generated by the same models. This shows that there is a large variation, of up to $25 \%$, in the predicted neutrino fluxes. The disagreement in the ratio of near/far neutrino fluxes varies by up to $10 \%$. If this could be reduced to $2 \%$ it would be of 
the order of the systematic and statistical errors of the experiment.
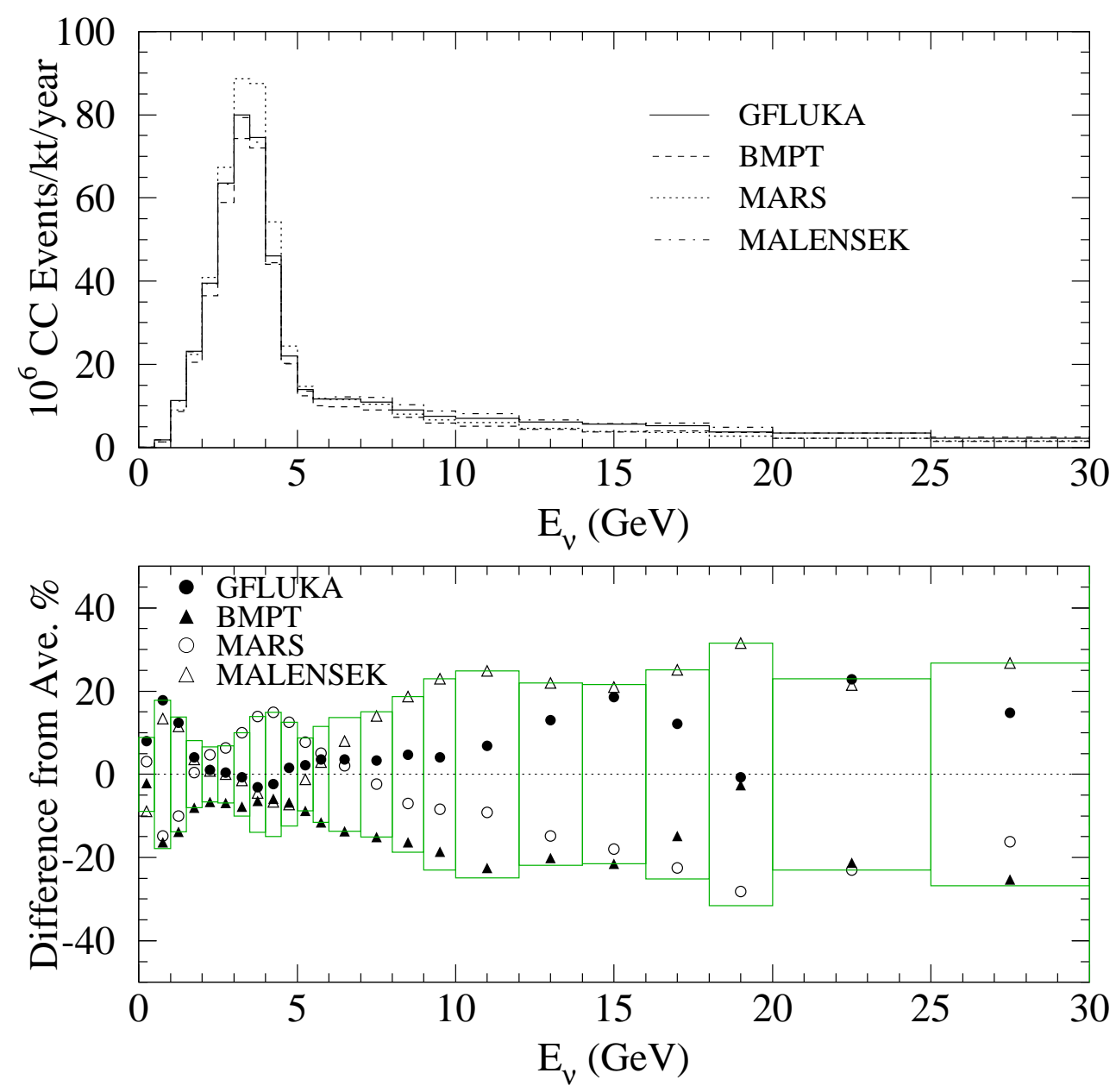

Figure 7.1: Predictions of absolute neutrino rates at the MINOS near detector using four hadron production models [68-71].

The neutrino beam in MINOS from the NuMI beam is generated by a combination of pions and kaons. Figure 7.3 shows the contribution to the neutrino flux from kaons. As seen, below $5 \mathrm{GeV}$ the contribution from kaons is small; however this quickly climbs to $30 \%$ at $30 \mathrm{GeV}$.

Figure 7.4 shows the relevant momentum ranges that contribute to the MINOS 

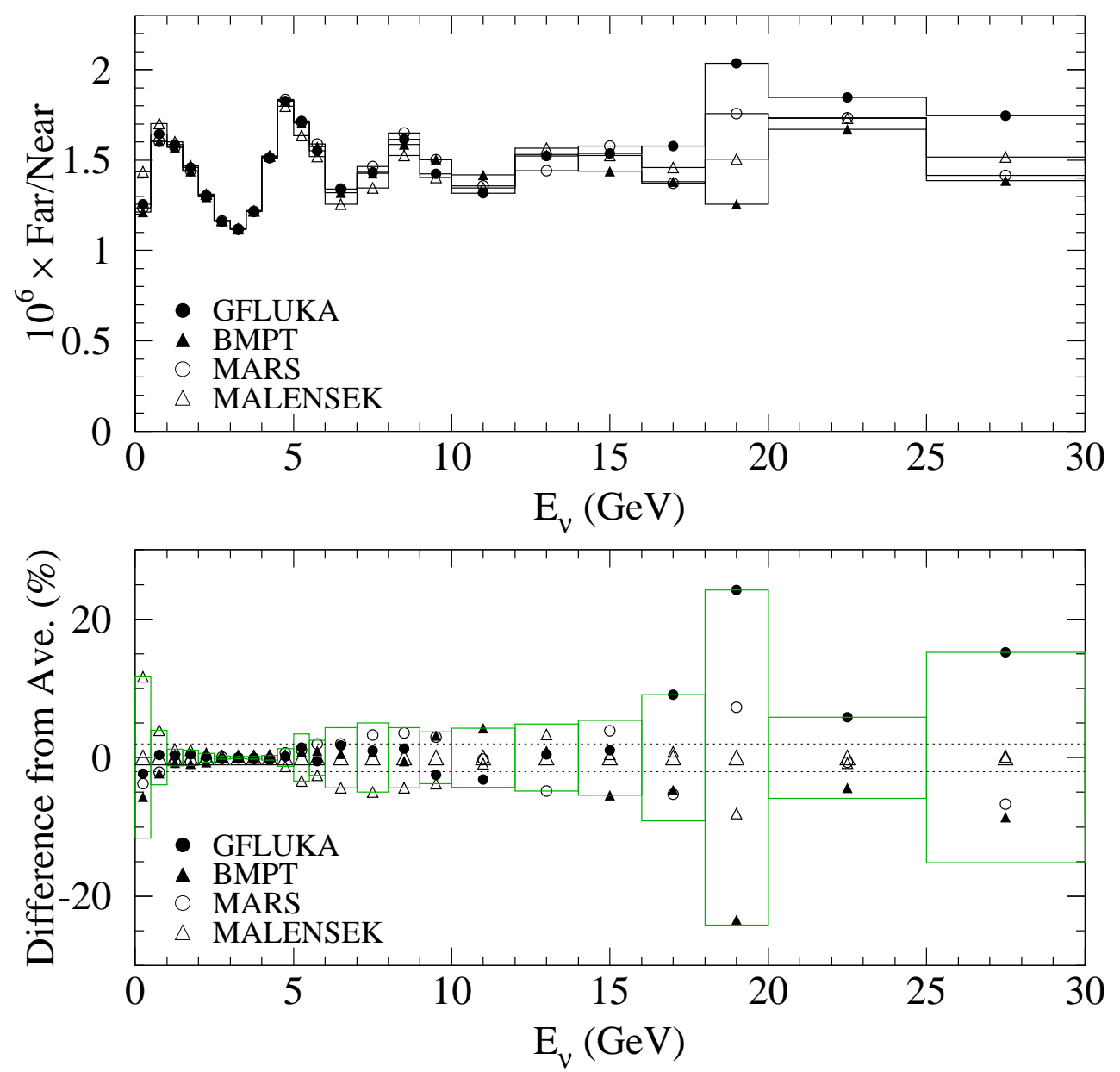

Figure 7.2: The ratio of near to far neutrino fluxes for the same four models as in figure 7.1. It is clear from these plots that, even though they agree at low energies, the models vary by as much as $25 \%$ for the absolute fluxes, and as much as $10 \%$ for the ratio at higher energies. 

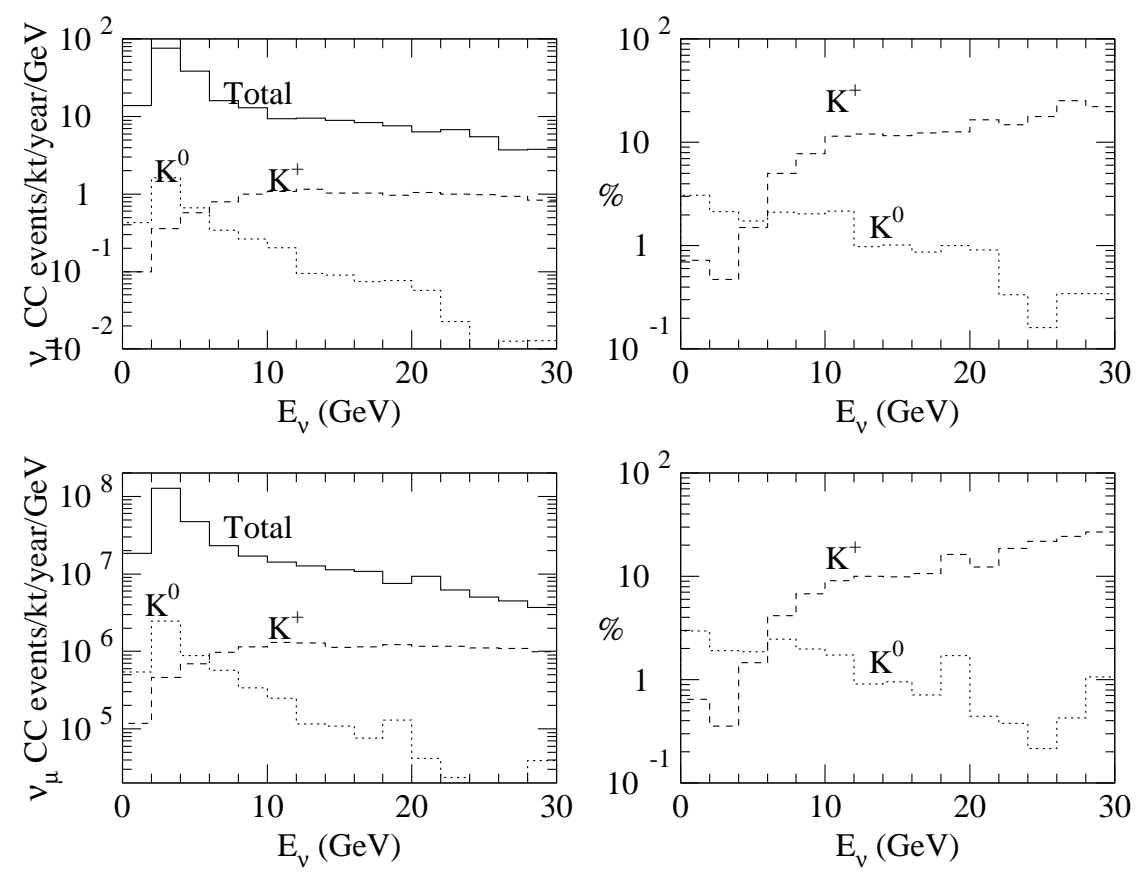

Figure 7.3: Kaon contributions to the muon neutrino flux from the NuMI beam. The top plot shows data from the far detector, the bottom plot for the near detector. It is clear that the kaon composition of the beam is of particular importance at higher energies. 
neutrino flux spectra, overlaid with the coverage of current data.

\section{Low Energy Beam}

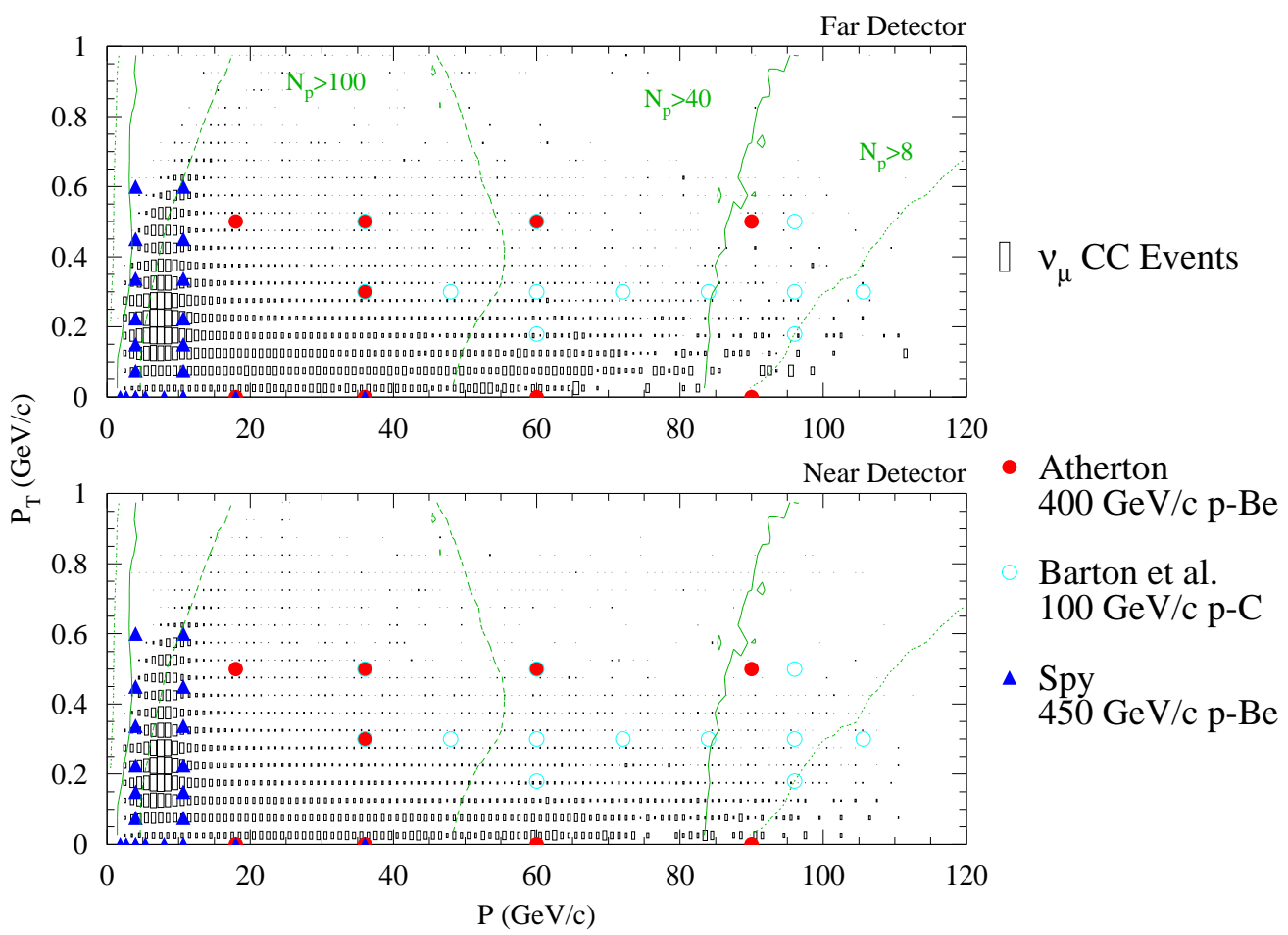

Figure 7.4: The distribution in $p$ and $p_{T}$ for secondary pions produced on the NuMI target. Overlaid are the locations of existing hadronic production measurements [72-74]. Also shown are the acceptance regions for the NA49 experiment, assuming at least 8,40 or 100 TPC track points.

As can be seen from the experimental results overlaid on this plot, hadron production data are rare. The SPY results [74] only have data in the forward momentum direction for particle production, except at 15 and $40 \mathrm{GeV}$ where they have covered a range of $p_{T}$. The Atherton data [73] although having a large forward $p$ range, were limited in the $p_{T}$ to 0 and $500 \mathrm{MeV}$. The Barton results [72] provide the best data as yet, with a wide range of forward $p$ values, however, there are still only two or three $p_{T}$ values per bin. The consequence of these results is that the hadronic produc- 
tion models have to extrapolate and interpolate the data from only 2 or sometimes 3 points. This leads to high errors.

\subsubsection{Atmospheric Neutrinos and the $K^{+} / \pi^{+}$Ratio}

The second motivation for the NA49 analysis was for the study of atmospheric neutrinos. The ratio of kaon and pion production from $\mathrm{p}+\mathrm{C}$ collisions is very important for the study of atmospheric neutrinos. It determines the available centre of mass energy, the decay chain and the flavour of neutrino produced. Figure 7.5 shows the proportion of upward produced muons at Super-Kamiokande produced by kaons. The kaons produce around half of the neutrino-induced muons. This is due to the combination of steeply falling primary cosmic ray spectrum, shown in figure 7.6, and the difference in available energy in the rest frame of the decaying meson.

Figure 7.7 shows the difficulty in modelling these hadronic production data. This plot, from [75], shows the changes introduced to the hadronic production model G-FLUKA by introducing the SPY data to create the FLUKA distribution.

The most precise current measurements of the $K^{+} / \pi^{+}$ratio are those from Atherton et al [73] and SPY [74], shown previously. Both measurements only have limited phase space regions, and used thick targets which were extrapolated to zero thickness for atmospheric calculations.

There were thin target measurements made by Barton et al [72] but with far less precision. Serpukov [76-78] also has results. Atherton et al showed that the $K^{+} / \pi^{+}$ratio can vary as much as a factor of two over $p_{T}$ and the ratio is strongly dependant on $p_{L}$. The NA49 apparatus is capably of measuring this ratio over a very large phase space, to an accuracy of roughly $10 \%$. 


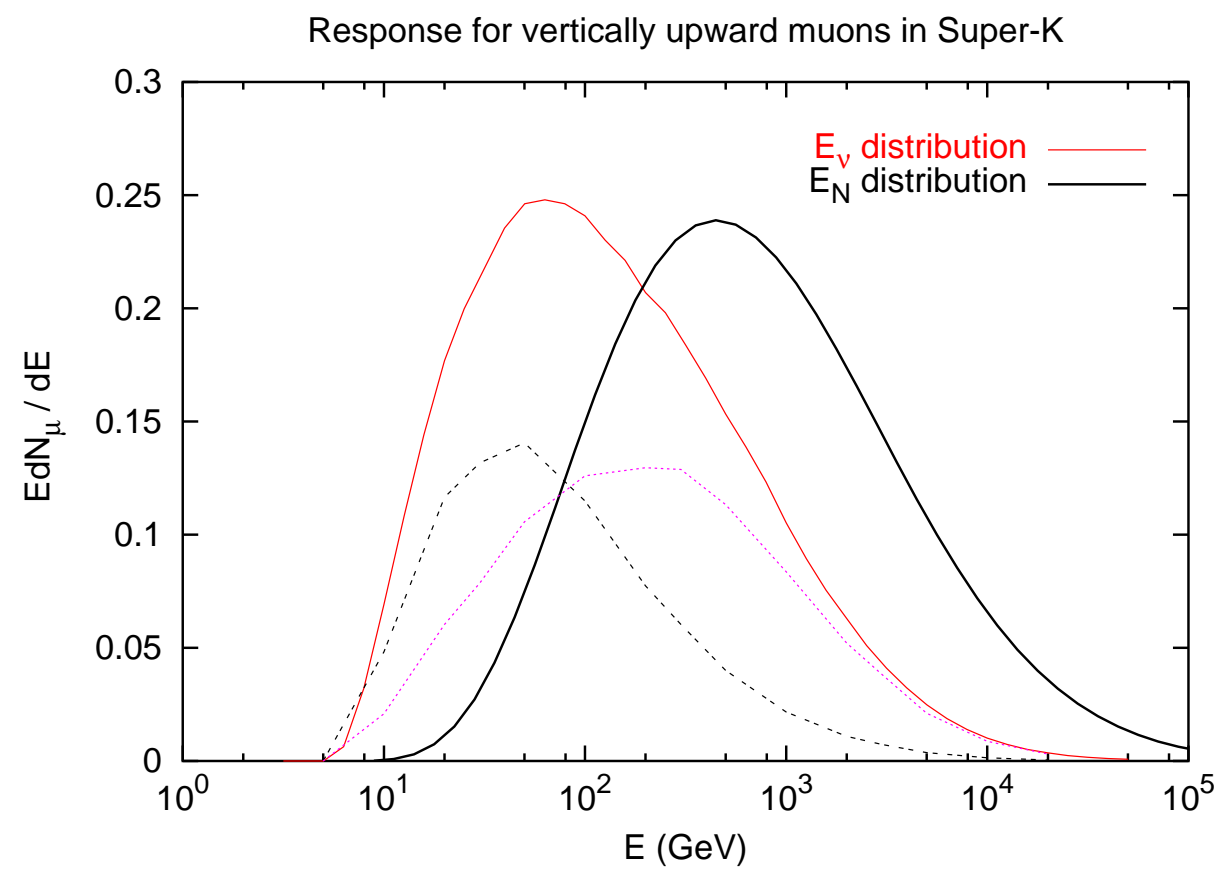

Figure 7.5: The distribution of neutrino energy $\left(E_{\nu}\right)$ and primary nucleon energy $\left(E_{N}\right)$ that produces the signal of through going upward muons at SuperKamiokande. The contribution to the neutrino energy from kaons (dashed) and pions (dotted) are shown. 


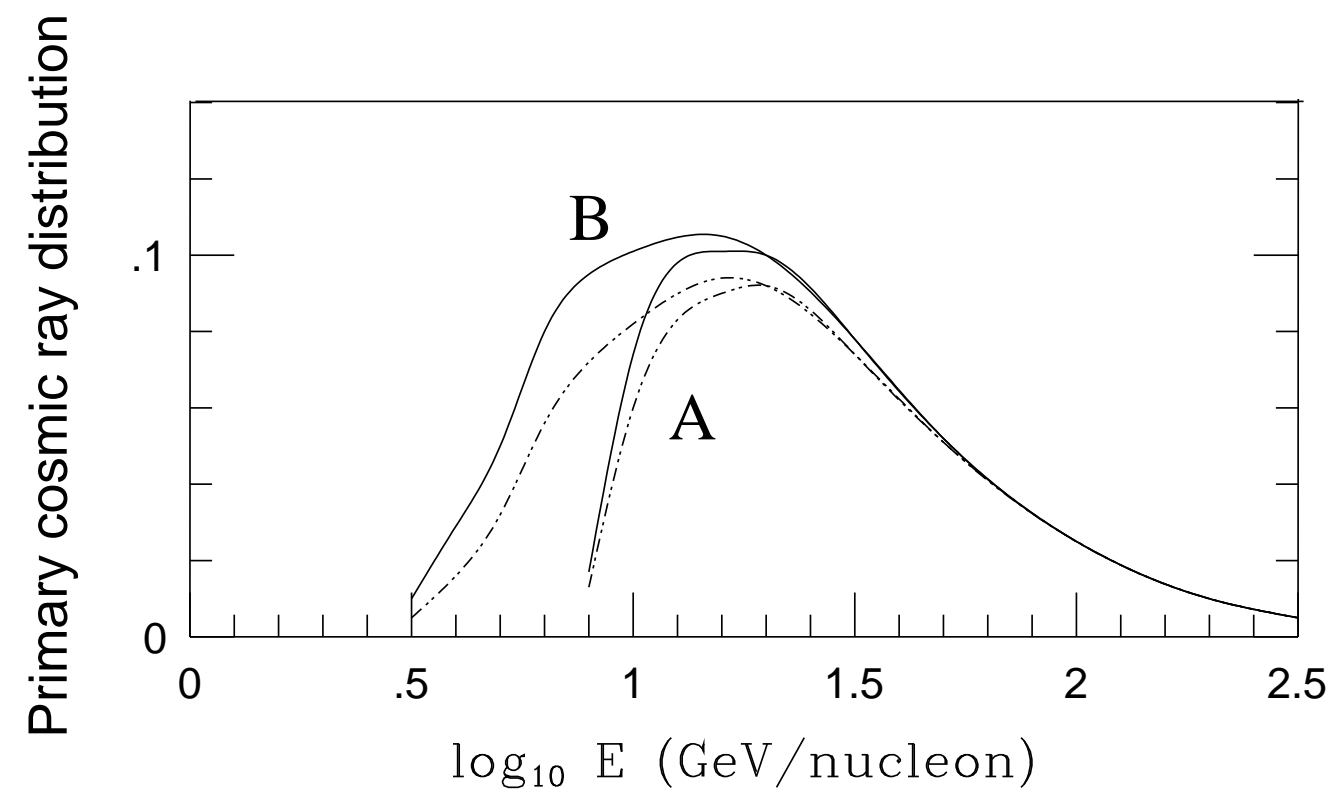

Figure 7.6: Energy distribution of the primary cosmic-ray nucleons giving rise to sub-GeV neutrino interactions in Super-Kamiokande. Curve A is for cosmic rays in the local atmosphere at Kamioka, neutrinos from above the horizon. Curve B is for cosmic rays giving rise to neutrinos from below the horizon. Each pair of curves shoes the variation of the signal between solar activity minimum (the upper curve) and maximum (the lower curve). 


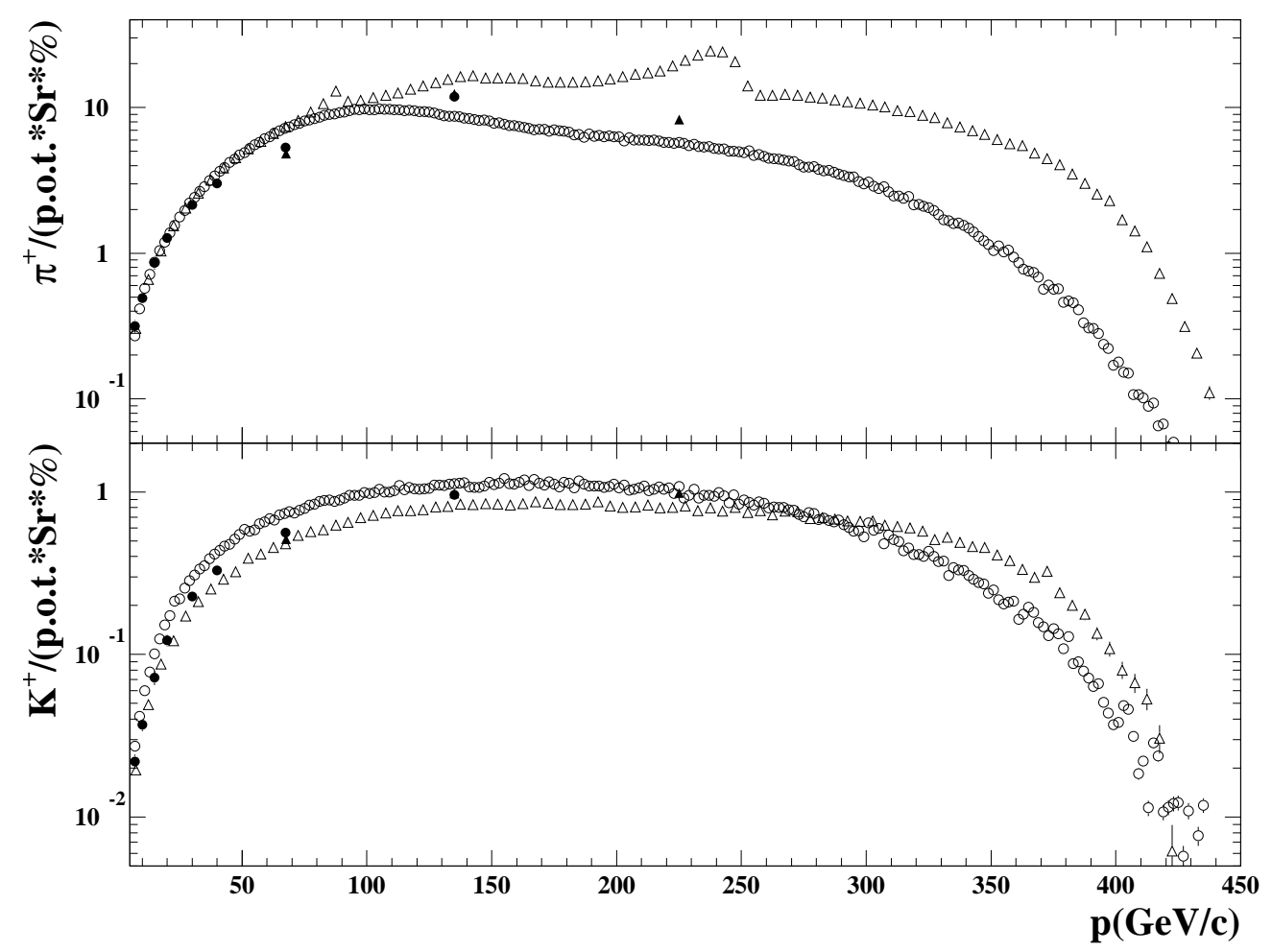

Figure 7.7: Existing model $\pi^{+}$and $K^{+}$distributions produced in $450 \mathrm{GeV} / \mathrm{c}$ protonberyllium collisions, for forward angles less than $0.2 \mathrm{mrad}$; open circles: FLUKA; open triangles: G-FLUKA; full circles and triangles: experimental data. 


\subsection{Experimental Apparatus}

The NA49 detector, Figure 7.8 is a wide-acceptance spectrometer that was designed for the study of hadron production in $\mathrm{p}+\mathrm{p}, \mathrm{p}+\mathrm{A}$ and $\mathrm{A}+\mathrm{A}$ collisions from the CERN SPS. The detector is mainly comprised of four large-volume Time Projection Chambers, TPCs, which carry out the tracking and particle identification from $\frac{d E}{d x}$ measurements. The data collected from the NA49 experiment should help greatly towards increasing the model accuracy of the beam generation. This is due to its $100 \%$ acceptance within a $100^{\circ}$ azimuthal wedge around the magnet bending plane.

Figure 7.9 shows the region of space in the $p, p_{T}$ plane that is covered by the NA49 TPCs with different magnet and target configurations.

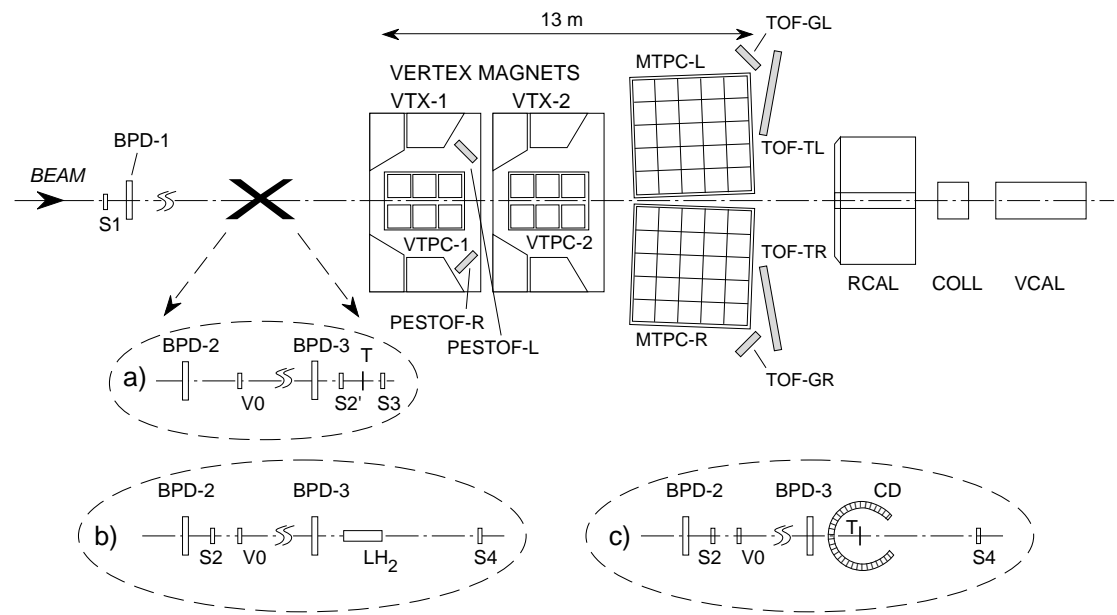

Figure 7.8: Schematic of the NA49 detector and beam information detectors (not to scale). Various beam detector configurations are shown for a) $\mathrm{Pb}+\mathrm{Pb}, \mathrm{b}) \mathrm{p}+\mathrm{C}$ and c) $\mathrm{p}+\mathrm{C}$ collisions. The target position is at the front face of the first Vertex Magnet (VTX-1). Configuration c), for $\mathrm{p}+\mathrm{C}$ collisions, was used in our data run.

In the region of interest for these hadronic production models, theory suggests that the distributions evolve slowly with beam energy. Due to this the original plan was to perform several runs at differing beam energies with the NA49 experiment. However, due to time and financial constraints, only two runs where performed, 

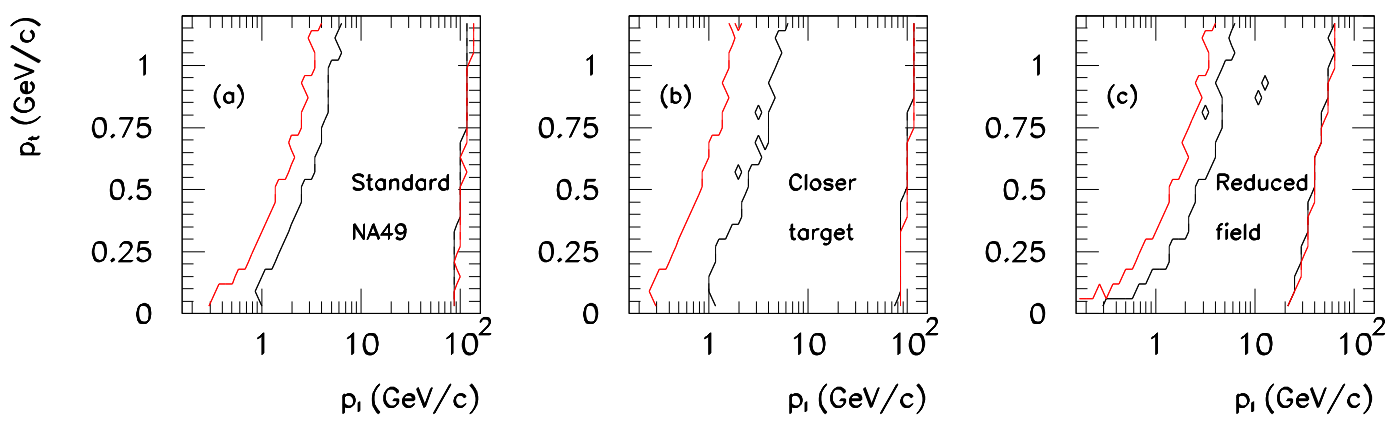

Figure 7.9: $p, p_{T}$ phase space covered by the NA49 p-C experiment in a $100^{\circ}$ wedge around the bending magnets. The lighter, red line signifies an acceptance requiring only 8 hits in the TPC, whereas the darker black line shows the acceptance for a higher number of hits, 30. Three situations are shown: a) Normal target and magnet mode. b) Target moved $60 \mathrm{~cm}$ closer to the first TPC. c) Magnetic field reduced by a factor of 4. Configuration a) was used; this provided the greatest coverage in the limited time.

at $100 \mathrm{GeV}$ and $158 \mathrm{GeV}$. Unlike the previous experiments, which needed several measurements to cover the $p, p_{T}$ region at each energy, a single run with NA49 covered nearly the whole of the phase space shown in figure 7.9.

\subsubsection{The SPS Accelerator Beam}

The NA49 experiment is situated in the North Area of the CERN complex. The protons used in this experiment are first generated in linear accelerators. They are then injected into the Proton Synchrotron Booster where they become accelerated to $95 \mathrm{MeV} / c$. The Proton Synchrotron further increases the momentum to $4.5 \mathrm{GeV} / \mathrm{c}$. After final acceleration in the Super Proton Synchrotron the particles enter the lab at a maximum momentum of up to $450 \mathrm{GeV} / \mathrm{c}$. Upstream scintillation detectors and beam position detectors provide precise information about the timing, charge and position of the incoming particles. The transverse position of incoming beam particles are measure using a telescope of beam detectors placed along the beam line, 
shown in figure 7.8 as BPD-1/2/3. These detectors are small $\left(9 \mathrm{~cm}^{2}\right)$ proportional chambers.

Interactions in the target are selected by anti-coincidence of the incoming beam particle with a small scintillation detector (S4 in figure 7.8).

\subsubsection{Magnets}

There are two super-conducting dipole magnets employed in the NA49 detector. They have a combined power of 9 Tm at currents of around $5000 \mathrm{~A}$ and are centred on the beam line. The centres of the two magnets are based at $2000 \mathrm{~mm}$ and 5800 $\mathrm{mm}$ from the target. Each magnet has a $1000 \mathrm{~mm}$ gap between the upper and lower coils, in which are placed Time Projection Chambers (TPCs). To be able to operate TPCs within magnetic fields, one either needs an extremely homogeneous field, or to have very precise knowledge of the magnetic field magnitude and orientation.

In the case of NA49 there is very precise knowledge of the field properties. This was performed in two ways. Detailed field measurements were taken with Hall probes on a small scale grid with cell sizes of $4 \mathrm{~cm}$ spacing. Also the magnetic field was calculated by TOSCA code, these two methods agreed to within $0.5 \%$. The magnets cover a volume of $16.5 \mathrm{~m}^{3}$.

\subsubsection{Time Projection Chambers}

There are four Time Projection Chambers (TPCs) in the NA49 experiment, two are in series in the beam line, known as Vertex TPCs (VTPCs), and two are further down the beam line either side of the centre on the horizontal plane, these are known as the Main TCPs (MTPCs). All the TPCs are shown in figure 7.8 and are labelled as VTPC-1, VTPC-2, MTPC-L and MTPC-R. This figure also shows how the MTPCs are tilted to align with the spread of the beam.

The TPCs are divided into 62 readout chambers, each $72 \times 72 \mathrm{~cm}^{2}$. These detectors are built with the same detector technology as many TPCs. A charged particle 
travelling through the gas within a readout chamber ionises some of the gas, releasing electron clusters. These clusters drift in the ambient electric field towards a gating grid followed by a cathode plane. Signals are picked up by pads on the back cathode plane. Figure 7.10 shows a schematic cross section of this. The pad sizes in the TPCs are small, down to $3 \mathrm{~mm}$, allowing up to 4000 channels per readout module.

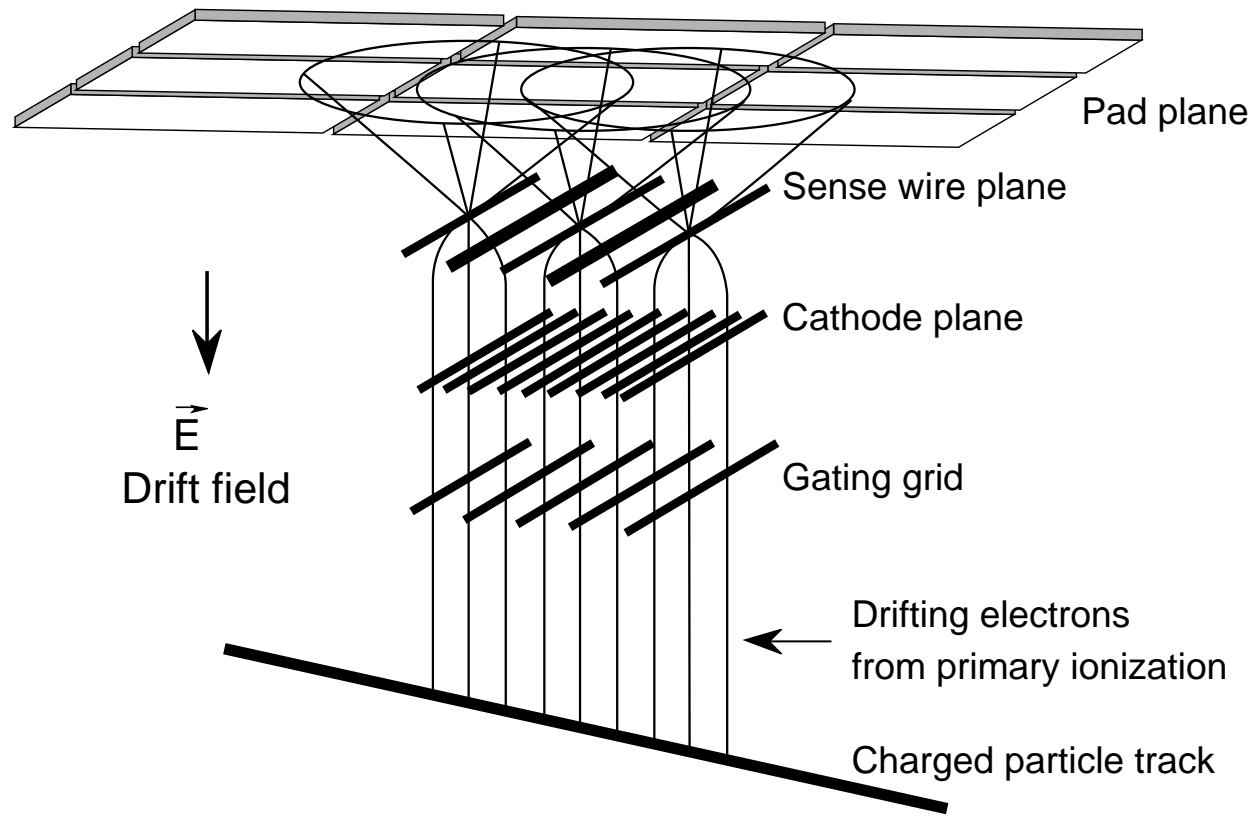

Figure 7.10: Schematic of the TPC readout chambers. The drift direction is vertically upwards.

Due to the kinematics of fixed-target collisions at SPS energies, TPCs are very efficient at particle detection. The mean particle emission angle is only in the region of $100 \mathrm{mrad}$, as shown in the example event in figure 7.11. Most particles are therefore contained within the TPCs, with only $100 \mathrm{~cm}$ drift lengths on average.

The magnets do however provide a large transverse momentum kick of the order of $1.5 \mathrm{GeV}$. This allows particles of opposite charge to be cleanly split into two hemispheres. This kick provides a change in track angles of a few degrees for high energy particles, to $\sim 50^{\circ}$ for low momenta particles. Due to this high transverse 


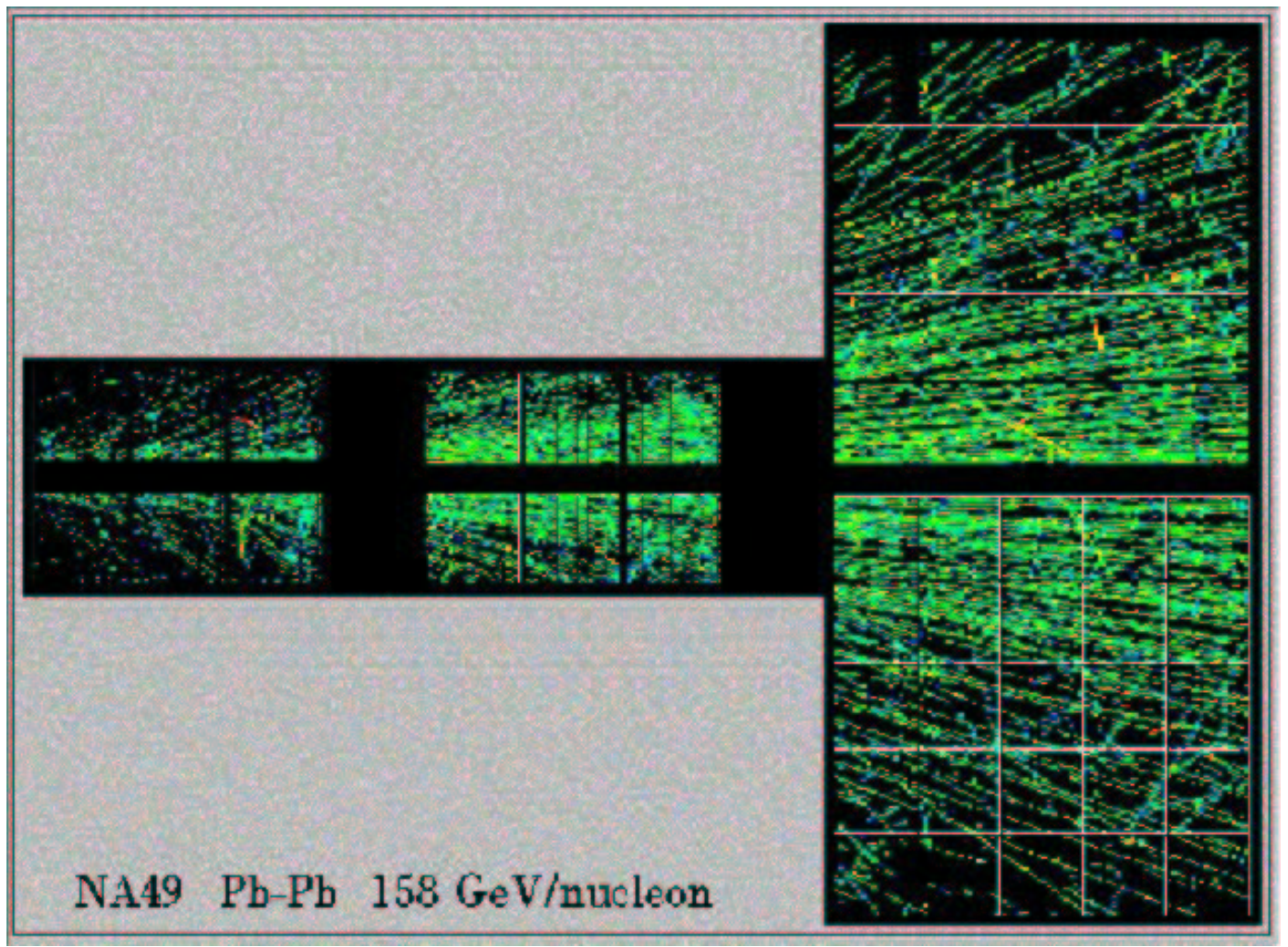

Figure 7.11: An example from the NA49 event display. Shown is an event from a $158 \mathrm{GeV} \mathrm{Pb}+\mathrm{Pb}$ run. Only a $7 \mathrm{~mm}$ vertical slice around the beam centre is shown to improve clarity. The multiplicity on these lead-lead events is far higher than any seen with $\mathrm{p}-\mathrm{C}$ interactions. 
track bending, the lowest energy particles detectable in the first VTPC are around 1 $\mathrm{GeV} / \mathrm{c}$. As each momentum particle has its own individual path through the TPCs, the readout pads are twisted to the same path direction as the particles. This keeps the angles between tracks and pads small.

It must be kept in mind that the NA49 detector was built for far higher multiplicities from $\mathrm{Pb}+\mathrm{Pb}$ collisions than are seen from $\mathrm{p}+\mathrm{C}$ collisions. For this reason charge information from the sense wires is not recorded, as the data flow was too high. Therefore tracking and energy loss measurements are only possible via pad readout.

Table 7.1 shows some properties of the TPCS, and table 7.2 shows some geometrical properties of the NA49 TPC system.

\begin{tabular}{|l|c|c|}
\hline \multicolumn{1}{|c|}{ Property } & VTPCs & MTPCs \\
\hline \hline Gas mixture & Ne/CO2 & Ar/CH/CO2 \\
Gas ratios & $90 / 10$ & $90 / 5 / 5$ \\
Diffusion coefficients & $220 \mu \mathrm{m} / \sqrt{\mathrm{cm}}$ & $270 \mu \mathrm{m} / \sqrt{\mathrm{cm}}$ \\
Drift velocities & $1.4 \mathrm{~cm} / \mu \mathrm{s}$ & $2.4 \mathrm{~cm} / \mu \mathrm{s}$ \\
Drift length & $66.6 \mathrm{~cm}$ & $111.7 \mathrm{~cm}$ \\
\hline
\end{tabular}

Table 7.1: Properties of the TPCs

\subsubsection{Electronics}

The small pad sizes and large TPC areas in the NA49 detector lead to a very large number of readout channels, the order of 182,000 . Electronics were custom built to handle this flow of data. The front end electronics are grouped into 32 channel cards, each channel representing a corresponding pad on the cathode plane. The analog signal from these channels is amplified, shaped and time-sampled to be stored in a Switched Capacitor Array (SCA). This can be considered to be a series of 512 


\begin{tabular}{|c|c|c|c|c|}
\hline dimensions $[\mathrm{mm}]$ & VTPC-1 & VTPC-2 & MTPC-L/R & wire material \\
\hline width & 2000 & 2000 & 3900 & \\
\hline length & 2500 & 2500 & 3900 & \\
\hline height & 980 & 980 & 1800 & \\
\hline drift length & 666 & 666 & 1117 & \\
\hline pad length & 16,28 & 28 & 40 & \\
\hline pad width & 3.5 & 3.5 & $3.6,5.5$ & \\
\hline pad angles & $12-55$ & $3-20$ & 0,15 & \\
\hline $\mathrm{pad} / \mathrm{sense}$ wire dist. & 3 & 2 & 2,3 & \\
\hline sense wire diam. & 0.02 & 0.02 & 0.02 & W-Ru (gold plated) \\
\hline sense wire spacing & 4 & 4 & 4 & \\
\hline field wire diam. & 0.125 & 0.125 & 0.125 & $\mathrm{Cu}-\mathrm{Be}$ (gold plated) \\
\hline field wire spacing & 4 & 4 & 4 & \\
\hline Frisch grid wire diam. & 0.075 & 0.075 & 0.075 & $\mathrm{Cu}-\mathrm{Be}$ \\
\hline Frisch grid wire spacing & 1 & 1 & 1 & $\mathrm{Cu}-\mathrm{Be}$ \\
\hline gating grid wire diam. & 0.075 & 0.075 & 0.075 & $\mathrm{Cu}-\mathrm{Be}$ \\
\hline gating grid wire spacing & 1 & 1 & 1 & \\
\hline
\end{tabular}

Table 7.2: Geometrical dimensions of the NA49 TPC systems. 
capacitors, each with sample and hold circuitry. These charges are then transfered to an ADC with 9 bit resolution. These data then transfer to the counting house via optical cables, where its stored for reconstruction, calibration and analysis. 


\section{Chapter 8}

\section{NA49 Data Reconstruction and Calibration}

\subsection{Acquisition}

The beam spills from the SPS occur every 25 seconds and last for 5 seconds. In our data taking run, we were restricted to $\sim 30$ events per spill. This was a design constraint resulting from the need of the NA49 detector to be able to handle the extremely high multiplicity events from $\mathrm{Pb}+\mathrm{Pb}$ collisions.

\subsubsection{Storage}

The NA49 data is stored by the DAQ onto large capacity magnetic tapes. The data is stored in a format named DS PACK. This is an object-orientated client-server data manager package. All the information from the detector, such as pressures, temperatures, beam positions and other parameters, are stored in Data Summary Tapes, DSTs, along with the actual detector physics data. The DST files are very large, and contain far more data than is normally needed for $\frac{d E}{d x}$ analysis. Therefore a reduced data format has been produced which allows quick and easy access to 
relevant data. These micro-DSTs are ASCII based files. They contain data such as run and event number, and information about the reconstructed tracks, such as track momentum, charge and measured $\frac{d E}{d x}$.

\subsubsection{Reconstruction}

A major and critical part of the work done by the NA49 collaboration on this data was the reconstruction. The data is written to the tapes in raw data format, and hence must be reconstructed by the following method;

\section{Cluster Finding}

The raw data from the TPCs represents a 3D distribution of digitised charge deposits, recorded in a format of pad row $(y)$, pad number within the row $(x)$ and time(z). As the charge produced from the ionised track diffuses as it travels, an entire cluster is read out by the readout electronics. This consists of the integrated charge, and the position is read as the centre of mass of the cluster. Hence, the cluster finder is the first step in the reconstruction, it effectively seeks the points of ionisation, and records the sum of the charge deposited by that cluster.

\section{Tracking}

The track finding algorithms pieces together the points left from the cluster finding algorithm into tracks. As the relative positions of the TPCs are known very accurately (better than $200 \mu \mathrm{m}$ ) global tracking can take place by gluing together the separate tracks from individual TPCs. If the tracks pass through either of the magnetised VTPCs the momentum of the particle can be measured from the curvature of the track. The tracking process also reduces singles and event independent external muons or cosmic rays.

\section{Vertex Resolution}

The vertex of the primary collision is calculated from the reconstructed tracks. The vertical and horizontal components are known to a large accuracy from the recorded 
beam position. The accuracy of the other vertex fit parameter depends greatly on the multiplicity of the event. Due to the target construction on occasion there are events that are the result of collisions with the carbon target holder. If the multiplicity is too low, then it becomes impossible to distinguish these events from collisions with the target, as shown in figure 8.1.

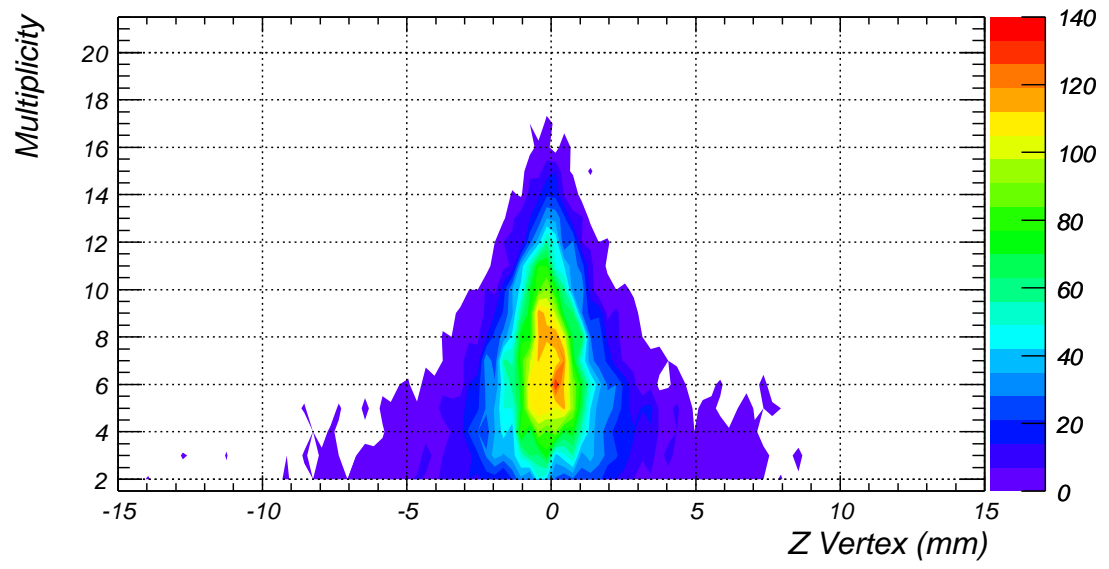

Figure 8.1: Plot showing the dependence on multiplicity of the $\mathrm{z}$ vertex position. It can be seen here that at low multiplicities there are events that do not have their vertex fitted with the target. The target was $1 \mathrm{~cm}$ long centred on zero.

For a certain fraction of events, with low multiplicity, vertex reconstruction is not possible. Due to this the NA49 collaboration have developed weighting factors according to the multiplicity. These weighting factors are implemented in the final $\frac{d E}{d x}$ fitting routine.

\section{2 $\frac{d E}{d x}$ Calibration}

The post-reconstruction calibration is extremely important for study of the $K^{+} / \pi^{+}$ ratio. Figure 8.2 shows the results from an 8 hour run, with no post reconstruction calibration; only the minimal calibration involved in reconstruction has been ap- 
plied. At this stage there seems to be very little structure to the curve, and it is very difficult to make out any particle identification. However, the basic structure of the Bethe-Bloch curve can be seen.

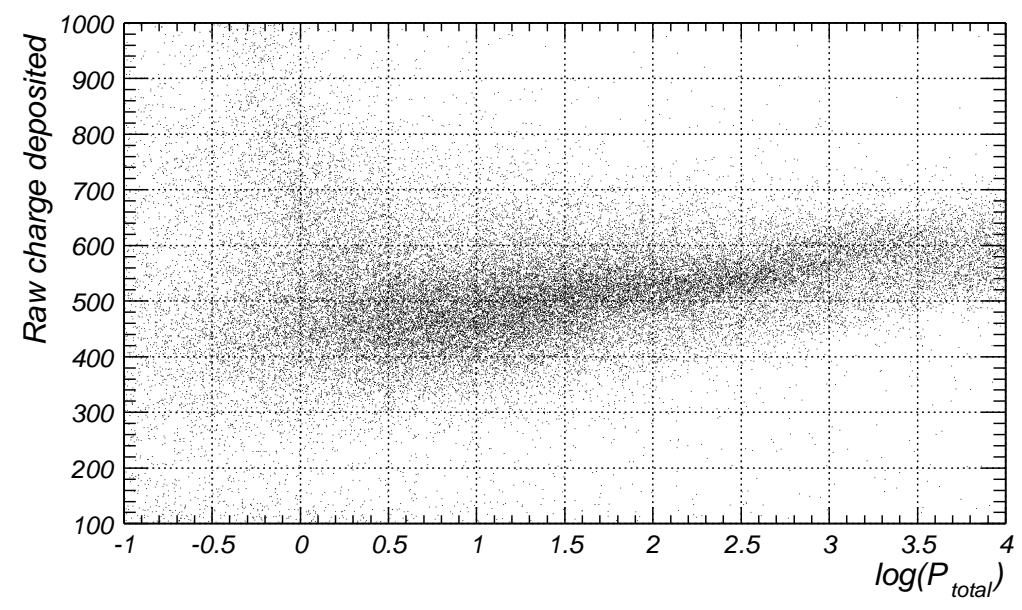

Figure 8.2: This plot shows the Bethe-Bloch curve for post calibration data from run 4797 . The vague shape can be seen; however, any serious attempt at particle identification at this point is impossible.

I will outline the various methods used to calibrate the data, and give an indication on the effectiveness of each method.

\subsubsection{Track Corrections}

There are two types of track correction made in the calibration procedure. The first is to consider the difference between the pad length along the track, and the actual track length. This is shown in Figure 8.3

The pads in all four TPCs are aligned parallel to the most probable momentum paths of the tracks, in an attempt to minimise this effect. However, there are still track angle differences, and these must be accounted for. Look up tables were developed to allow the relevant angle of each pad to be found, and compared with 


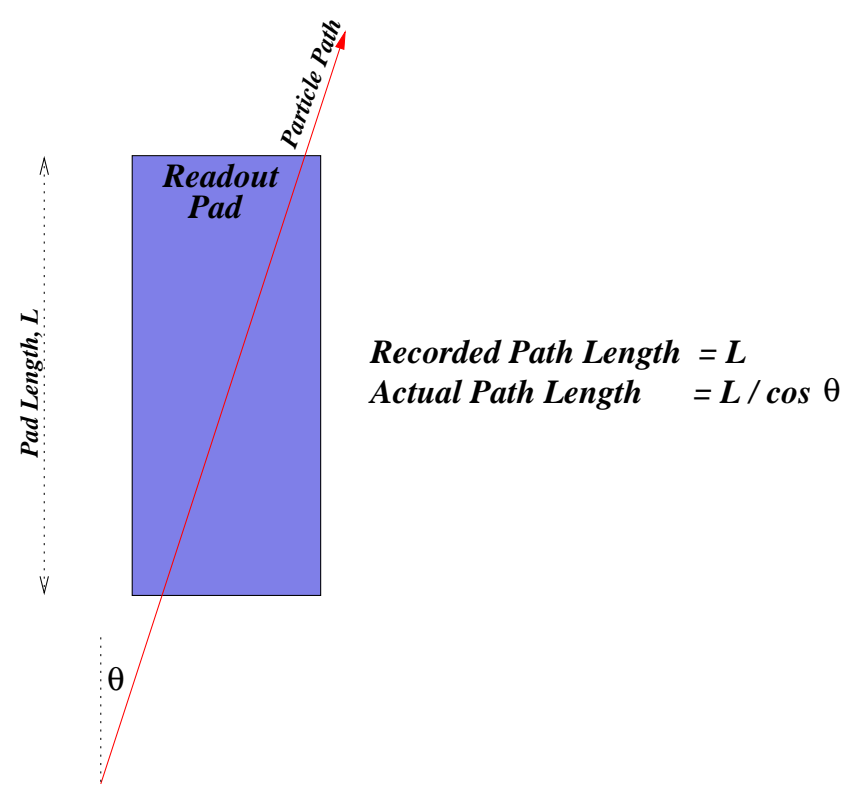

Figure 8.3: Schematic showing the difference between the assumed track length and the actual track length. The pads in the TPCs are twisted in line with the standard particle paths to reduce this effect as much as possible. 
the track angle at that point. Any correction is then trivial to apply to the $\frac{d E}{d x}$ value at that point.

Further correction has to be done when "associating" the tracks from different TPCs together. There are different gases in each TPC, and also different gases in each section of the Main TPC's (MTPC's). The gain depends upon the gas being used. This results in a different so called "gas gain" factor depending upon track position. This also affects the $\frac{d E}{d x}$ value at each point, and therefore the $\frac{d E}{d x}$ values are varied accordingly.

\subsubsection{Sector Calibration}

There are two means of calibrating the detector on a sector-by-sector basis. The first, which is carried out bi-monthly, involves pumping a gas isotope into the TPC and analysing the produced spectrum for gain shifts. For this purpose ${ }^{83} \mathrm{Kr}$ is used; it produces electron deposits from $9-42 \mathrm{KeV}$. This is perfect for the NA49 TPCs, as the gain of the NA49 TPCs and pad sizes imply that a minimum ionising particle (MIP) will produce between 2 - $10 \mathrm{KeV} /$ pad. This calibration method also allowed the spread of gas within the TPC chambers to be analysed. Figure 8.4 shows the method used in more detail. This form of calibration is typically done before and after each SPS beam run of the detector. The time evolution of the detector response between these calibration runs has been seen to be uniform over the 62 sectors.

There are however other sector by sector variations in the pad responses on the shorter time scale of a day. This is mainly due to pressure variations within the chambers, and uncertainties in the setting of the high voltage grid supply and low voltage electronics supply. These variations are accounted for via analysis of the data taken during a run. Within each sector, a mean response is calculated for all particles travelling through that sector. As the particle distribution is dominated by pions in nearly all momentum bins this mean should vary only very slightly with sector position. Figure 8.5 shows an indication of the variation of these calibration 

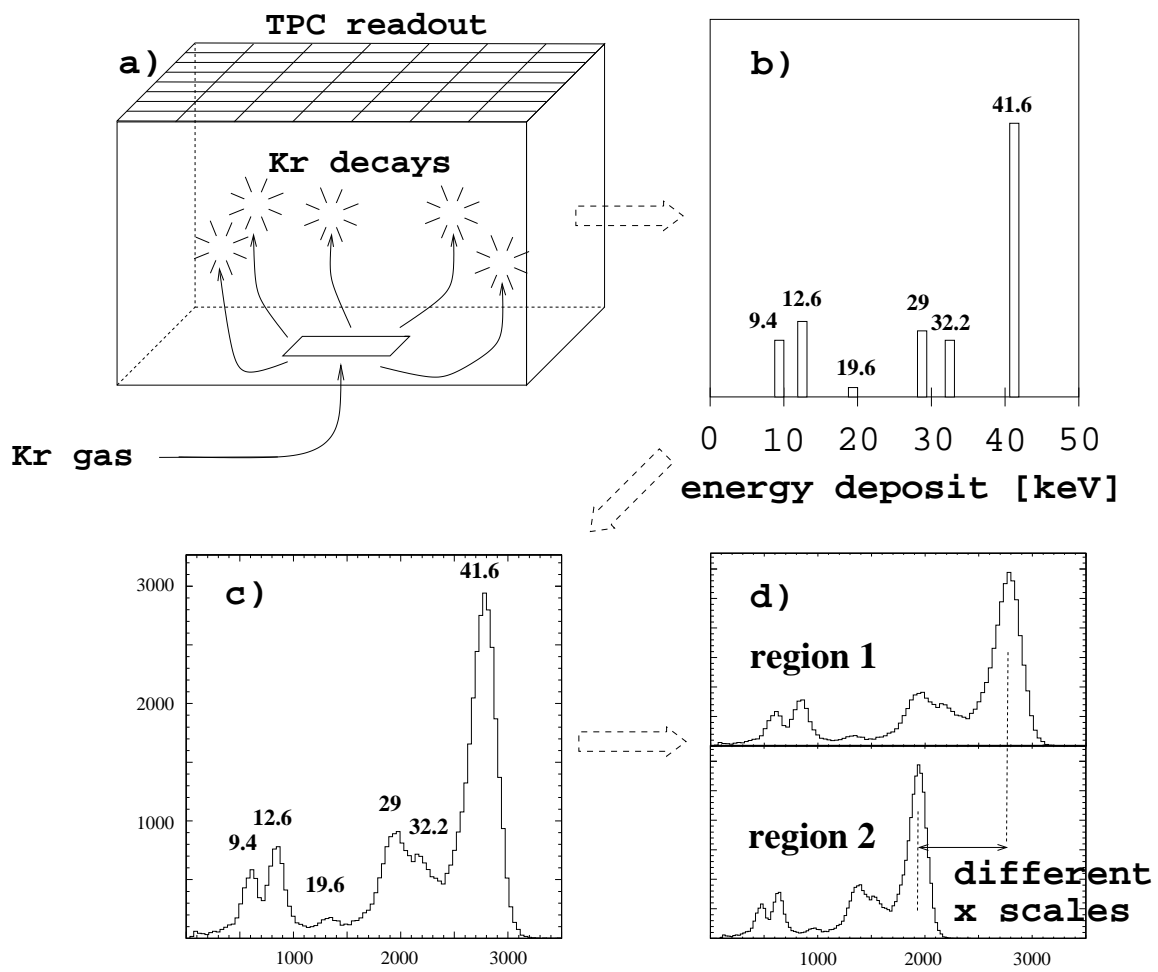

observed charge deposit [a.u.]

relative gain

Figure 8.4: This figure outlines the method used in the krypton calibration. [79]. First the krypton gas is pumped into the TPCs, this isotope decays with the energy spectrum shown in b). This produces the corresponding charge peaks in c). Each sector within the TPC has its own charge spectra with known peaks, these can then be used to calculate the relative gain, as in $\mathrm{d}$ ). 
constants used.

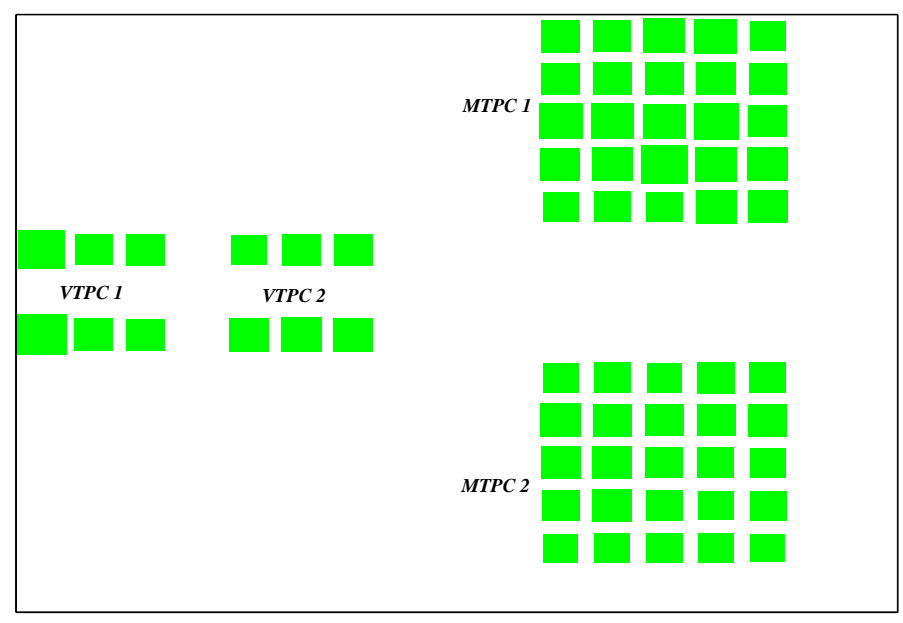

Figure 8.5: This is a schematic showing the relative gains of the 62 sectors of the TPCs in the NA49 detector. The area of the box represents the relative gain of that sector within its TPC. The first two sectors of the first VTPC on the left clearly show the effect of having different gas pressures, and hence very different gains to the other sectors within that TPC.

\subsubsection{Truncated Mean Correction}

The distribution of charges deposited on the TPC pads by a track of several meters or more is very nearly Gaussian. However, Monte Carlo analysis, as well as real data analysis, has shown that the $\frac{d E}{d x}$ resolution can be improved by up to $20 \%$ by considering truncated means.

The optimum truncation method proved to be discarding the upper $50 \%$ of charges, leaving only the lowest $50 \%$. This provided a method of stabilising the data from outlying points Figure 8.6 demonstrates this truncation. The resolution can then be parameterised as $38 \% / \sqrt{N_{c}}$ where $N_{c}$ is the number of clusters on the track. Figure 8.7 shows the distribution of the number of clusters per track $\left(N_{c}\right)$. 
Considering the range of track lengths in this plot, it implies a $\frac{d E}{d x}$ resolution from the truncated mean of around $6 \%$ for tracks which only pass through VTPC1. Going to $3 \%$ for tracks which pass through both VTPC's and a MTPC.

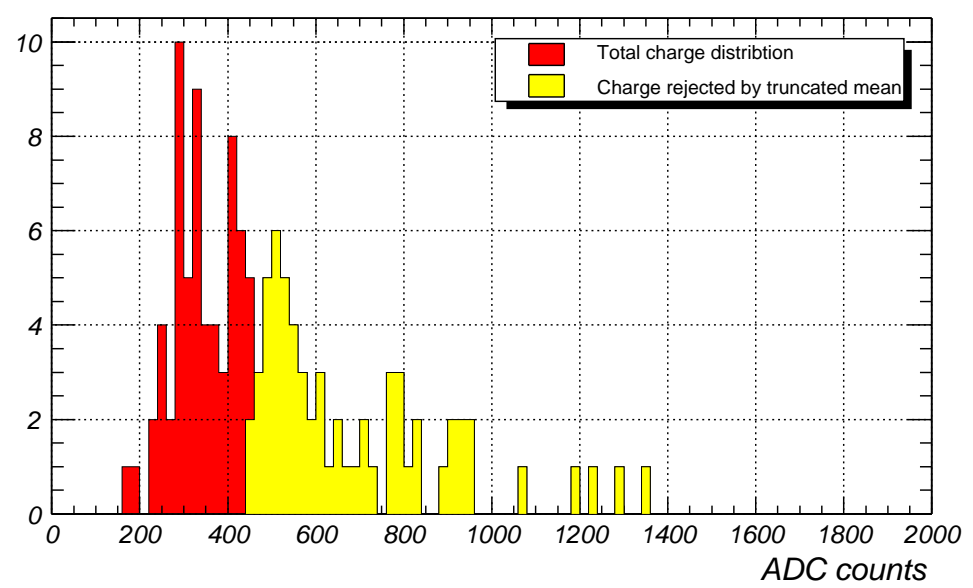

Figure 8.6: Shown here is the charge distribution from a typical track which passes through 3 TPCs. The yellow area of the histogram shows the region that is rejected by the truncated mean, following the method described by the Monte Carlo.

\subsubsection{Drift Length Correction}

The biggest correction to be made in the $\frac{d E}{d x}$ calibration is that for drift length. As well as the electron attachment due to the gas properties, which accounts for about $1-2 \%$ of loss in the MTPCs and 3-4\% loss in the VTPCs, there is a loss due to diffusion. As a cluster drifts towards the readout electronics its width increases due to diffusion of the charge cloud. Due to the electronic noise constraints on the front end boards, charge readings below 5 counts are suppresed. Therefore, as the cloud spreads, more of its contributions are below this suppression threshold, reducing the final integrated charge for that cluster. This effect creates an apparent charge loss of around 5\%. However, when this effect is combined with the truncated mean method 


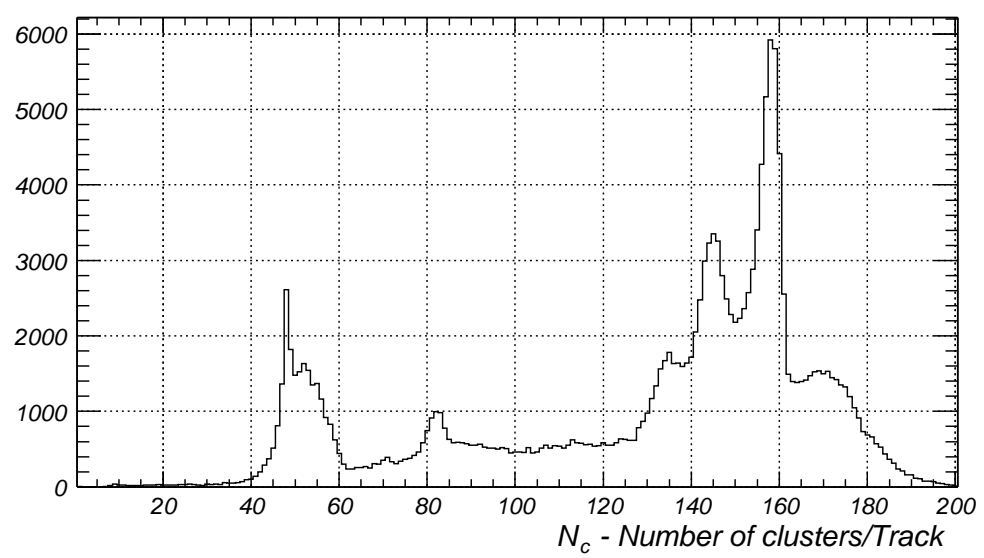

Figure 8.7: The number of clusters per track $\left(\mathrm{N}_{c}\right)$ for events from the NA49 detector. The peaks represent scenarios where the tracks pass through different numbers of TPCs and pads.

above, this loss increases to $7 \%$ as only the smallest $50 \%$ of charges are maintained for integration.

\subsection{Particle Identification}

The main problem facing the calculation of $\mathrm{K}^{+} / \pi^{+}$is that of particle identification. The particle ID is done via a method of identifying the amount of charge deposited per unit length of the particle track, $d q / d x$ which is proportional to the energy loss $\frac{d E}{d x}$.

\subsection{1 $\frac{d E}{d x}$ Energy Loss}

Charged particles moving through matter lose energy through processes of ionisation and atomic excitation. This energy loss can be described by the Bethe-Bloch equation [52], 


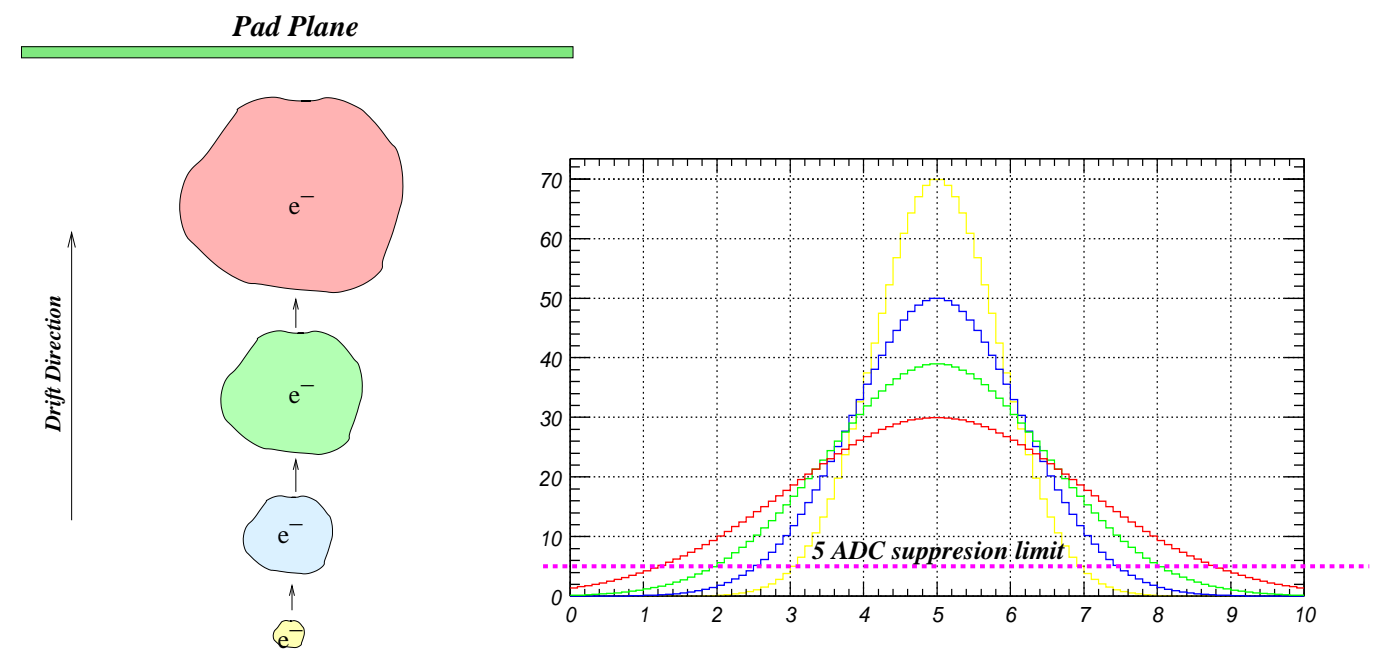

Figure 8.8: This shows the effect drift length has on the charge distribution that is corrected for by the drift length correction. The plot on the right shows an indication of the area the charge is spread over. It can be seen that as the cluster expands, a larger proportion of the charge is below the 5 ADC suppression limit, reducing the integrated charge value. 


$$
-\frac{d E}{d x}=K z^{2} \frac{Z}{A} \frac{1}{\beta^{2}}\left[\frac{1}{2} \ln \frac{2 \mathrm{~m}_{\mathrm{e}} \mathrm{c}^{2} \beta^{2} \gamma^{2} \mathrm{~T}_{\max }}{\mathrm{I}^{2}}-\beta^{2}-\frac{\delta}{2}\right]
$$

$T_{\text {max }}$ is the maximum kinetic energy which can be imparted to a free electron in a single collision,

$$
T_{\text {max }}=\frac{2 m_{e} c^{2} \beta^{2} \delta^{2}}{1+2 \gamma m_{e} / M+\left(m_{e} / M\right)^{2}} \approx 2 m_{e} c^{2} \beta^{2} \gamma^{2}
$$

for $2 \gamma m_{e} / M \ll 1$.

In the analysis of NA49 data a parameterisation of this determined by Gabor Veres is used, which allows for particle identification from $\frac{d E}{d x}$ information. Figure 8.9 shows momentum dependence of $\frac{d E}{d x}$ for several particles, generated by the NA49 parameterisation.

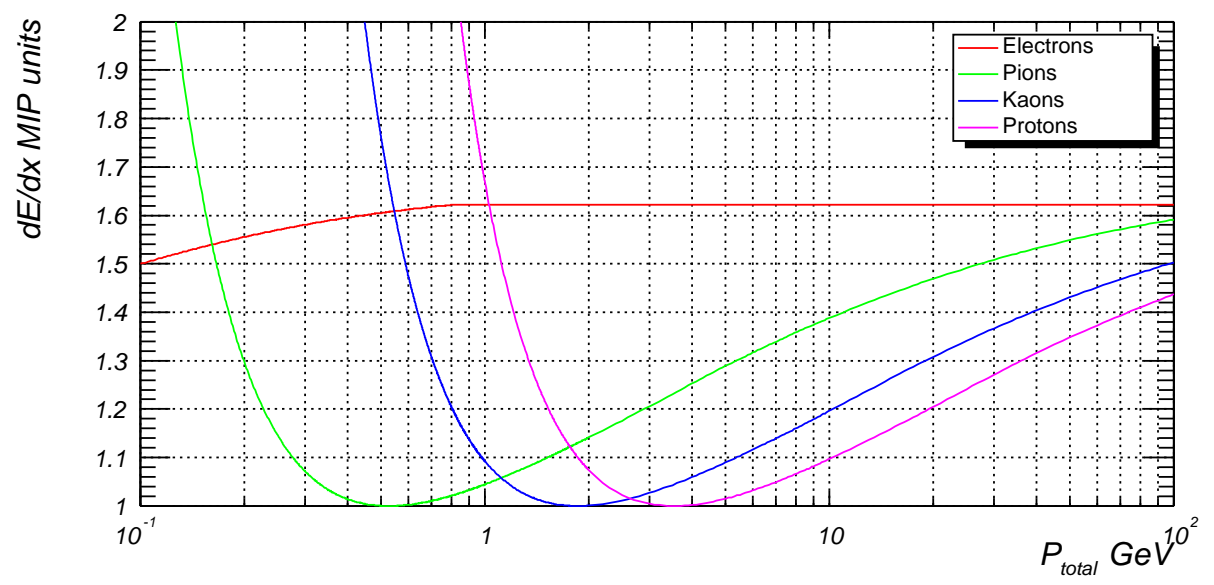

Figure 8.9: Bethe-Bloch distribution

The area of interest from this plot is in the relativistic rise. Only in this area, around $10 \mathrm{GeV}$ are the particle curves separated enough to allow particle identification. An ideal situation would produce the plot in figure 8.9, with four well defined distributions at each momentum bin from the raw data.

This is not the case however. Comparing plot 8.9 with figure 8.10 shows an 
indication of the problems. Figure 8.10 shows the $\frac{d E}{d x}$ distribution from all particles generated from the $158 \mathrm{GeV} \mathrm{p}+\mathrm{C}$ run at NA49.

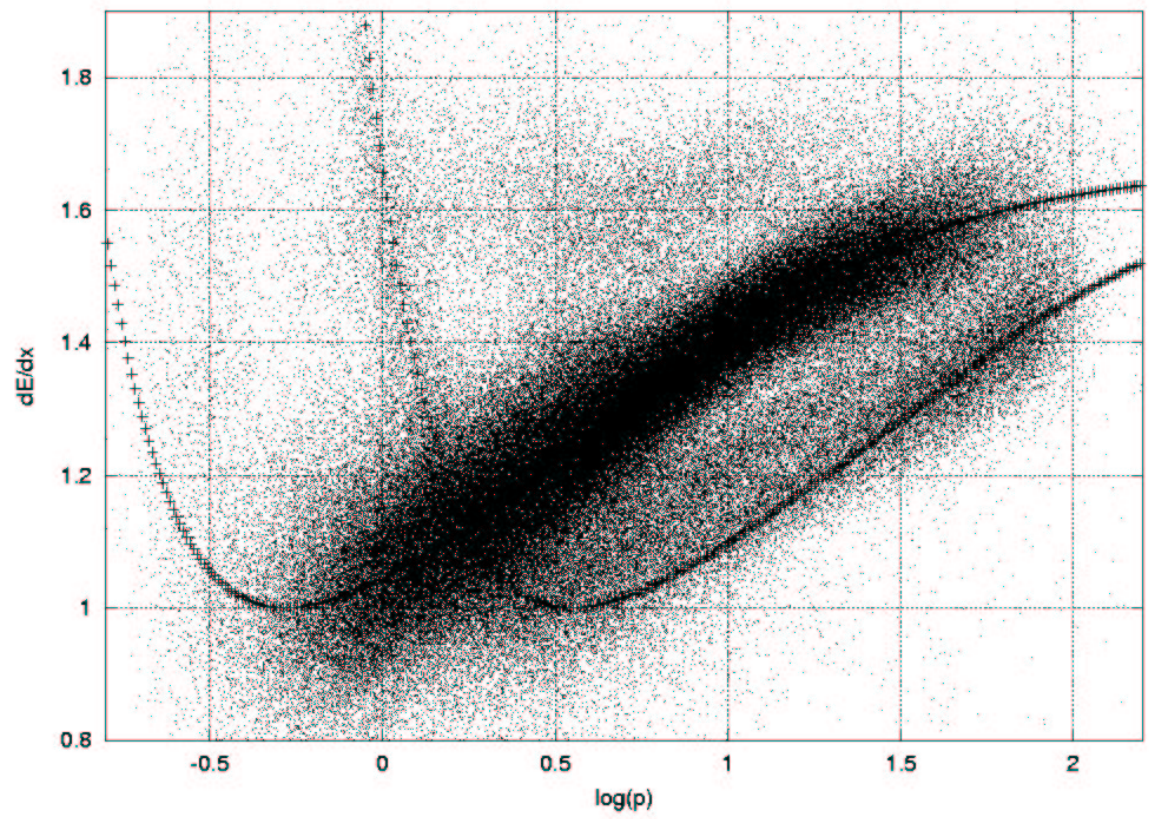

Figure 8.10: Bethe Bloch distribution from reconstructed $\mathrm{p}+\mathrm{C}$ data. The two functions shown by crosses represent the pions and kaons expected.

Shown in figure 8.10 are all particles generated from the reconstruction software. Excluded are tracks with poor vertex resolution and bad reconstruction parameters. The resolution of the particle distributions within the data set can be greatly increased by making sensible restrictions on the phase space included for analysis.

Cuts in the data had to be made to maximise the particle identification accuracy, as well as ensuring results were valid. Here I shall outline each cut as it was made, explaining the reason behind the restriction, and the effect it has on the data. 


\subsubsection{Physical Data Cuts}

These cuts were to ensure maximum acceptance of the data, and to ensure that all tracks accepted were fully understood:

- Charge cut

The first, and most obvious, restriction on the data was the charge cut. As we are interested in the $\mathrm{K}^{+} / \pi^{+}$ratio we can exclude all negative charged particles. This reduces the data set by around $50 \%$. As the strong magnetic field provide very large momentum kicks according to charge, this cut is trivial to make. Charge is assigned to each track during the reconstruction track finding stage.

- Sagitta cut

The next cut was related to one of the problems outlined in the $\frac{d E}{d x}$ calibration section. The pads in the TPCs are aligned parallel to the most probable momentum paths of the particles. This is to ensure that the maximum charge is collected from each path. However, this is only the case when the particle has the correct charge on the correct side of the detector. Figure 8.11 describes this in greater detail. This cut discards all events with a high transverse momentum in the direction opposite to their momentum kick.

- $\phi$ Cut

Figure 8.12 shows the relation between $\phi$ and $p$, it can be seen that the low end of the momentum range the acceptance in $\phi$ is restricted. To take account of this, there has to be an azimuthal angle cut, shown in figure 8.13. Events are only accepted that lie within a $100^{\circ}$ section of the detector, based around the horizontal beam plane. This ensures that the acceptance of the tracks is not affected by their momentum. Otherwise, at high momenta and large angles, the tracks would not travel through enough of the VTPCs to allow 


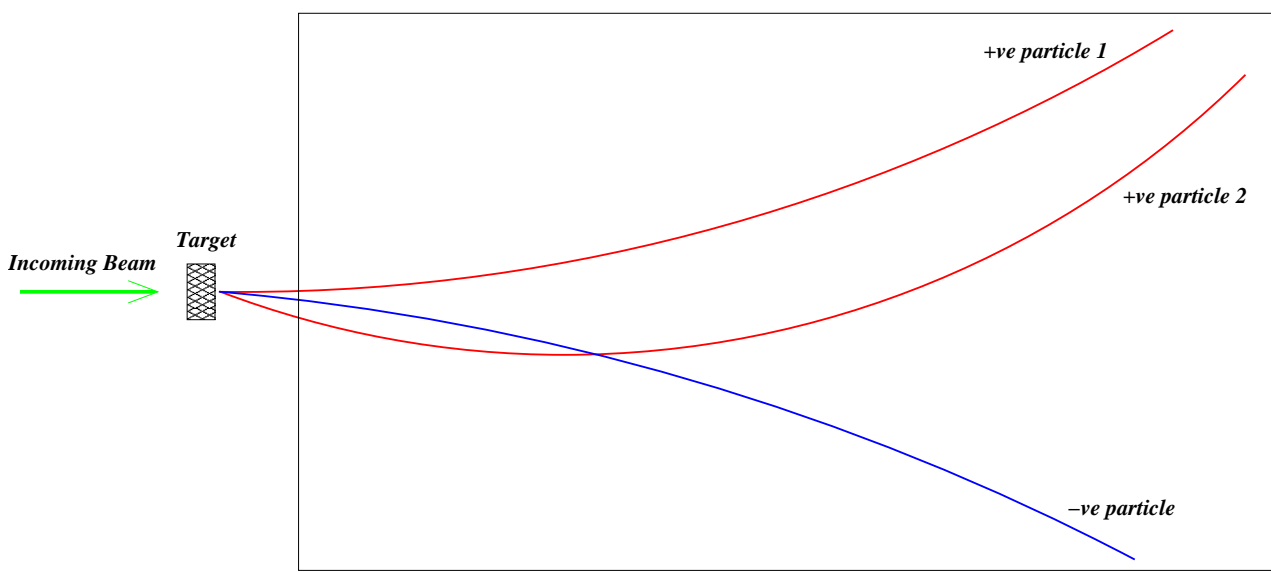

Figure 8.11: Schematic showing the sagitta cut. In this diagram, any positive particle from the interaction will be given a kick in the upward direction. +ve particles 1 and 2 have very similar forward momentum, however they have greatly different transverse momentum. This results in the second particle following a very different path than the first particle. As the pads are all tilted to follow a standard particle track with average transverse momentum, they are far from optimised for the tracks which follow very different paths such as that for particle 2 . . 

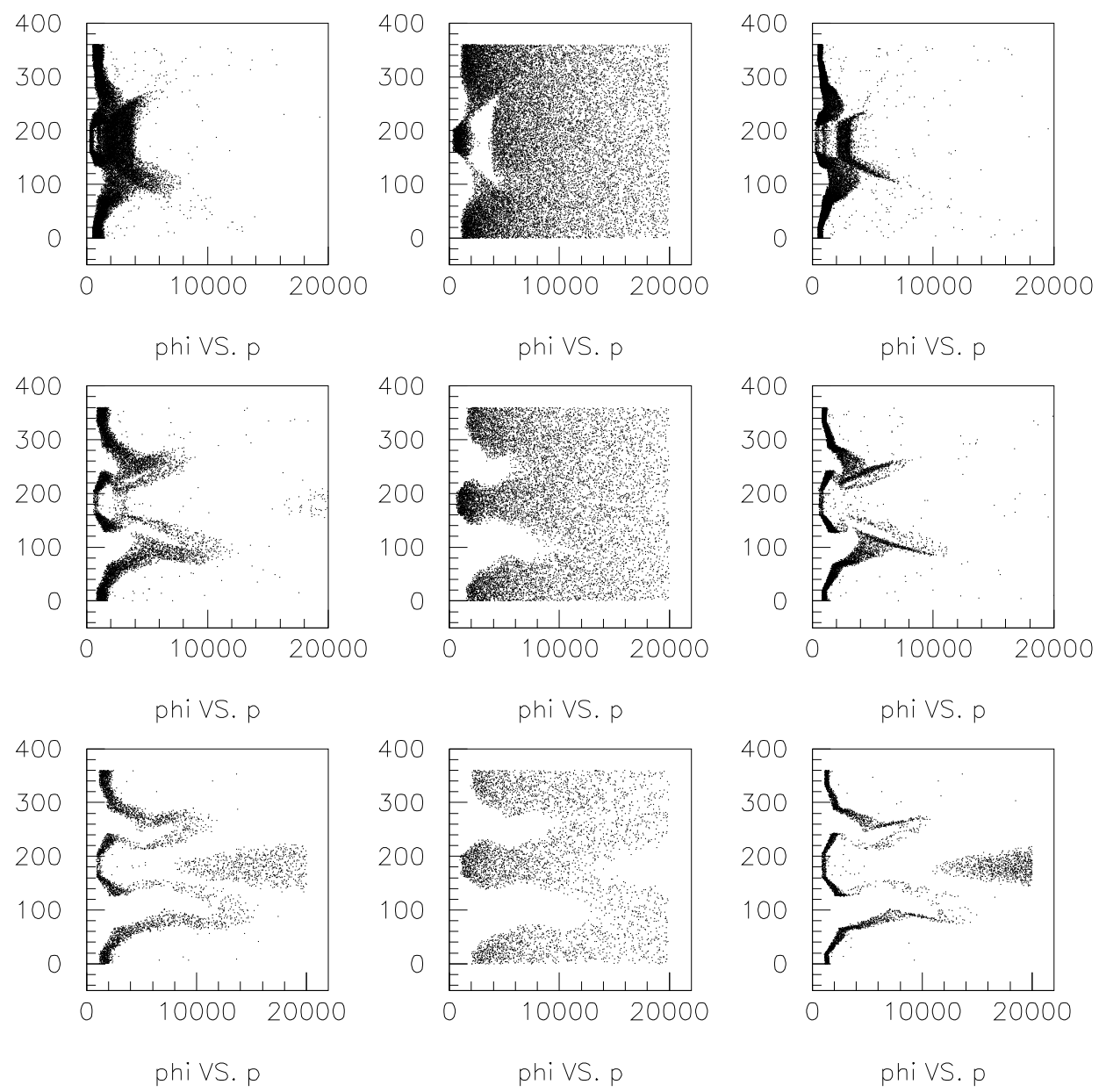

Figure 8.12: Scatter plots of $\phi$ vs $p$. The rows give different distributions in $p_{T}$ bins: top $0.0-0.2 \mathrm{GeV}$, middle $0.4-0.6 \mathrm{GeV}$, bottom $0.6-0.8 \mathrm{GeV}$. The left hand column shows events with less than 30 hits in the VTPCs, the middle plots show events with 30 or more hits in the VTPCs and the right hand ones show events with 15 or less VTPC hits. The middle column is relevant, as we only accept events with more than 30 hits in the VTPCs. 
proper reconstruction. This would therefore bias the data in favour of the low momentum tracks.

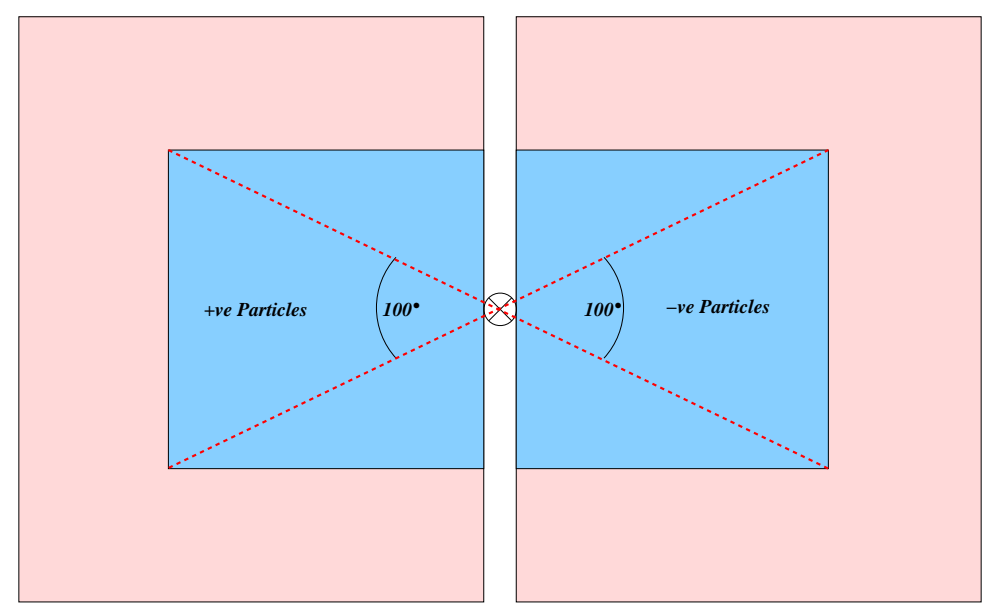

Figure 8.13: Due to the geometry of the detector a $100^{\circ}$ cut around the horizontal plane reduces acceptance problems. Otherwise we are limited to only low momentum events in the larger angle areas.

With these cuts it was ensured that the remaining data set would neither be biased towards any section of the detector, nor towards any energy or momenta.

\section{4 $\frac{d E}{d x}$ Fitting}

To calculate the absolute yields of the four main particle types, the data collected in any particular bin in $p_{T}, p_{L}$ phase space had to be fitted. Figure 8.14 shows an example of a fit to proton-proton NA49 data. The fit is comprised of four MC generated $\frac{d E}{d x}$ distributions, each assuming the particle is either a kaon, proton, pion or electron. The fit is then minimised by the MINUIT [81] package, adjusting the amplitude $\left(a_{j}\right)$ of each distribution to fit the experimental data;

$$
\left(\frac{d E}{d x}\right)_{e x p}^{h i s t}(i) \approx \sum_{j=1}^{4} a_{j} \cdot\left(\frac{d E}{d x}\right)_{M C_{j}}^{h i s t}(i)
$$




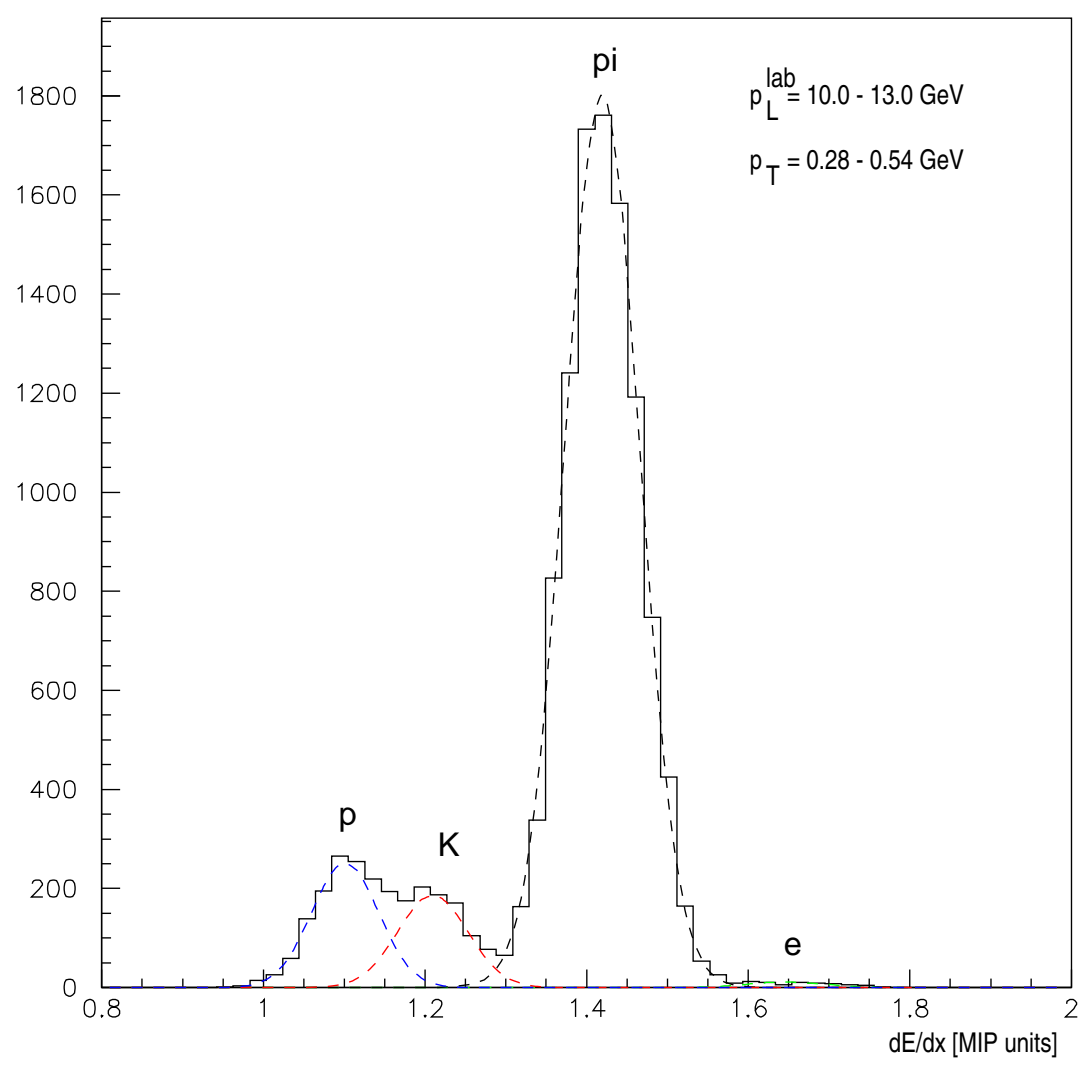

Figure 8.14: This histogram shows the measured $\frac{d E}{d x}$ and fitted MC simulated $\frac{d E}{d x}$ of positive charged particles within laboratory momentum $p_{L}^{l a b}=10.0-13.0 \mathrm{GeV}$ and transverse momentum $p_{T}=0.28-0.54 \mathrm{GeV}$. From [80]

Where $j \epsilon\{e, \pi, K, p\}$ are the four particle types. Therefore, if the total number of tracks $\left(N_{\text {tracks }}\right)$ in the bin is known, then the particle multiplicities $\left(N_{\text {particle }}\right)$ can be simply calculated by this number multiplied by $\alpha_{j}$.

The quality of the fit is measured by,

$$
Q=\frac{1}{N_{\text {tracks }}} \sum_{i=1}^{I_{\text {lim }}}\left[\left(\frac{d E}{d x}\right)_{\text {exp }}^{\text {hist }}(i)-\sum_{j=1}^{4} a_{j} \cdot\left(\frac{d E}{d x}\right)_{M C_{j}}^{\text {hist }}(i)\right]^{2}
$$

where $I_{l i m}$ is the number of histogram bins. Note that this $Q$ does not have the statistical significance of the common $\chi^{2}$. It provides an effective and quick method of measuring the goodness of the fit. 


\subsubsection{Monte Carlo Simulation of $\frac{d E}{d x}$}

The Monte Carlo simulation has the task of generating the $\frac{d E}{d x}$ distributions required by the above fitting technique. Each particle passing through and leaving a track in the detector is either a kaon, proton, pion or electron. Therefore at each momentum there is not a continuous range of probable $\frac{d E}{d x}$ but only four discrete values, one for each particle type. For each track the MC generates four values for $\frac{d E}{d x}$ based on the Bethe-Bloch parameterisation of each particle.

The corresponding MC error measurement can then be derived from the experimental measurement error. As $\frac{d E}{d x}$ measured for each track is the weighted mean of the separate $\left(\frac{d E}{d x}\right)_{i}$ measured in each TPC. Then the corresponding $\sigma_{e x p}^{\frac{d E}{d x}}$ of the track is a combination of the relative $\sigma_{i}$ 's from each TPC. The $\sigma_{i}$ of $\frac{d E}{d x}$ is very dependant on the energy loss in each TPC, which TPCs the tracks passed through, and the number of tracks in the TPC,

$$
\sigma_{i} \propto \frac{1}{\sqrt{N_{i}^{t r}}}\left(\frac{d E}{d x}\right)_{i}^{\alpha_{i}}
$$

Where $\alpha$ is dependant upon TPC and varies as $\alpha_{1}=\alpha_{2}=0.5, \alpha_{3}=0.7$ for the three types of TPC, and $N_{i}^{t r}$ is the number of the tracks points located in the $i$-th TPC. However, these values of $\alpha$ are known to be very inaccurate. We can therefore use this information to generate an estimated error for each MC track generated from the actual data, without too larger increase in error, by taking $\alpha=0.5$. As we have generated four different particles for each track recorded, and since the tracks $\sigma_{e x p}^{\frac{d E}{d x}}$ corresponds to the measured ionisation, we have to rescale the error according $\frac{d E}{d x}{ }_{M C}$

$$
\sigma_{M C}^{\frac{d E}{d x}}=\sigma_{e x p}^{\frac{d E}{d x}} \cdot \sqrt{\frac{\left(\frac{d E}{d x}\right)_{e x p}}{B B(p / m)}}
$$

This results in generating four MC events with $\frac{d E}{d x}$ centred around the BetheBloch value for the tracks momentum, with a corresponding $\sigma$, for each track in the experimental data, one for each of the four particles. 


\subsection{2 $\frac{d E}{d x}$ Fitting Corrections}

There are several corrections made to the MC generated distributions before they undergo fitting:

- Bethe-Bloch Shifts - The method used to generate the Bethe-Bloch distribution, and therefore the means of the MC generated histograms, is based on an estimated parameterisation. Therefore, it is sensible to allow some variation of the Bethe-Bloch used for the generation of the $\frac{d E}{d x}{ }_{M C}$ distributions for each particle. This has the ability to shift the means of each particles MC generated distribution

- Sigma Relative Shifts - Also, $\sigma_{M C}$ is based on the original experimental distribution being Gaussian. However we know this is not the case, also $\sigma_{\text {exp }}$ is modified by the truncated mean method. Therefore we allow some variation $\sigma_{r e l}$ for fine-tuning the distribution widths. Each particle's MC generated distribution is adjusted by this same width.

- Weight Factors and $\sigma_{\text {vertex }}$ - As shown in figure 8.1 the lower the number of tracks in an event the greater $\sigma_{\text {vertex }}$, the deviation of the fitted vertex, becomes. Sometimes in very low multiplicity events the vertex cannot be reconstructed at all and so these events must be dropped. There also occur bad events in which the reconstruction program cannot reconstruct any tracks at all, these are known as zero-prong events. In both these cases we have still triggered on the event, so they must be corrected for. Because of this, weighted factors were developed, by H.G. Fischer and T. Tsussa of the NA49 collaboration, to compensate for the missed events as a function of the multiplicity of the event. 


\subsection{3 $\frac{d E}{d x}$ Fitting Method}

The fitting method employed in finding the particle yields involves a mixture of computer automated fitting with human intervention for fine-tuning the corrections mentioned above. Figure 8.15 outlines the process involved in fitting a binned data set. The fitting software used is based on a FORTRAN program written by H.G.

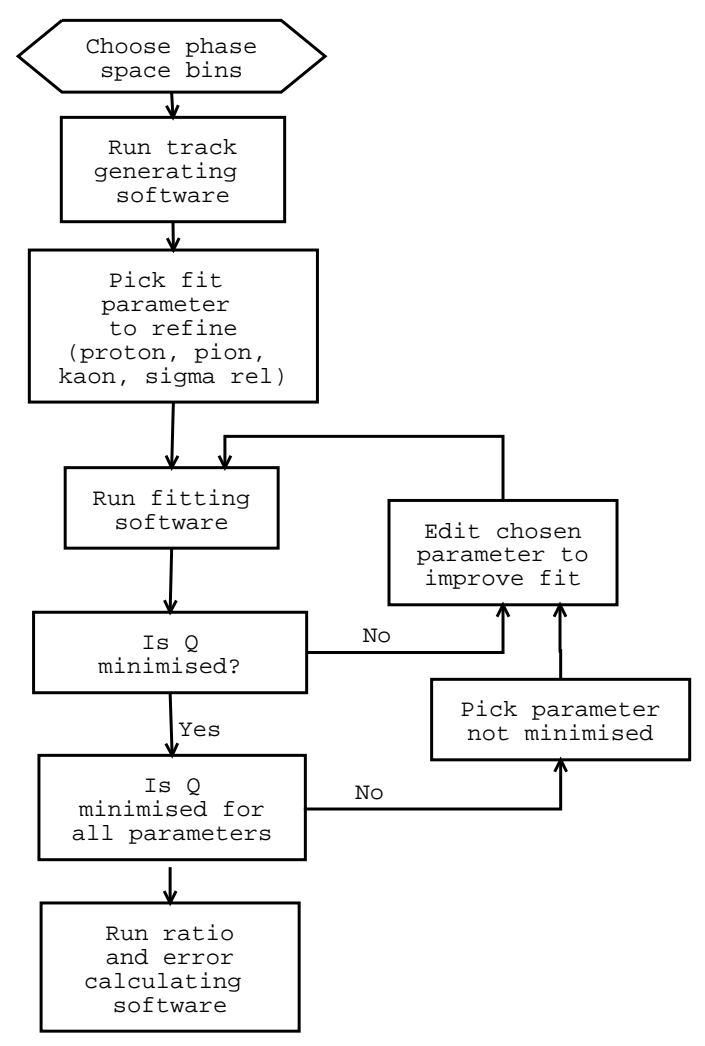

Figure 8.15: $\frac{d E}{d x}$ fitting algorithm.

Fischer. Initially, all the corrections are set to zero and the fitting program carries out a preliminary fit. This may produce a fit similar in fashion to the one shown in figure 8.16. The shifts to the MC generation are then applied. As these shifts and $\sigma_{r e l}$ are functions of the particles momentum, and are not independent, if they are set as free parameters the fitting routine often blows up and produces unphysical results. An example of this is shown in figure 8.17

As shown by figure 8.15 each of the Bethe-Bloch shifts and $\sigma_{\text {rel }}$ are altered in 


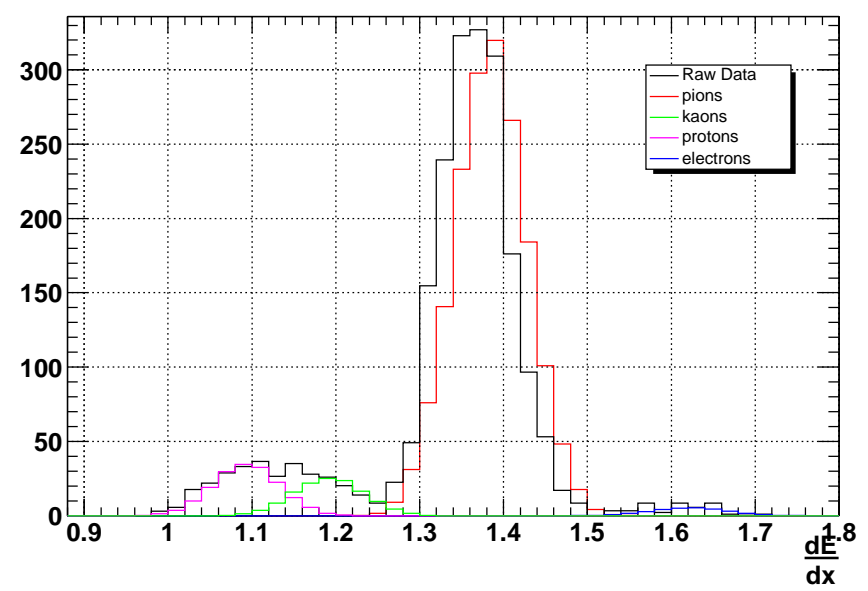

Figure 8.16: Initial fit produced by $\frac{d E}{d x}$ fitting routines. The need for the means of each particles distribution to be shifted can be seen, as well as the width being too narrow.

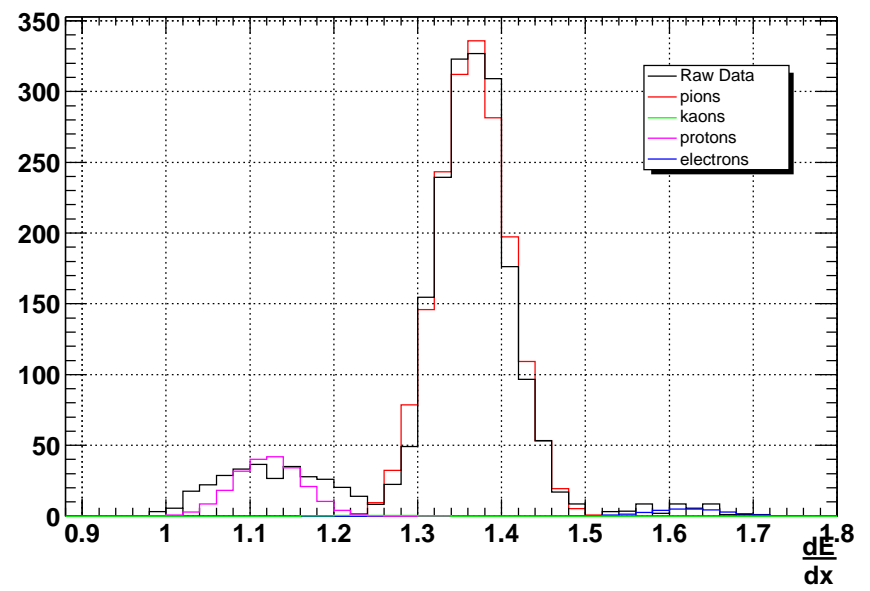

Figure 8.17: This plot demonstrates the situation which can occur if the BetheBloch corrections are set as free parameters for the minimising routine to calculate. The kaon yield has been reduced to zero to enable the proton peak to be fitted. 
turn until the quality of the fit, $Q$, is minimised. This method of manually fitting the data is very time consuming, using the original FORTRAN code would take 8-10 minutes for each of the 300 data sets analysed. To decrease this time I wrote a graphical interface to the FORTRAN code to facilitate the necessary manual adjustments, this is shown in Appendix $\mathrm{C}$ along with other fitting details.

When a reasonable fit has been acquired the yields of each particle type are recorded, along with the variations used for each fitting parameter. Figure 8.18 shows the result of each separate particles contribution. Figure 8.19 shows the comparison between the raw data and the sum of the different particle fits.

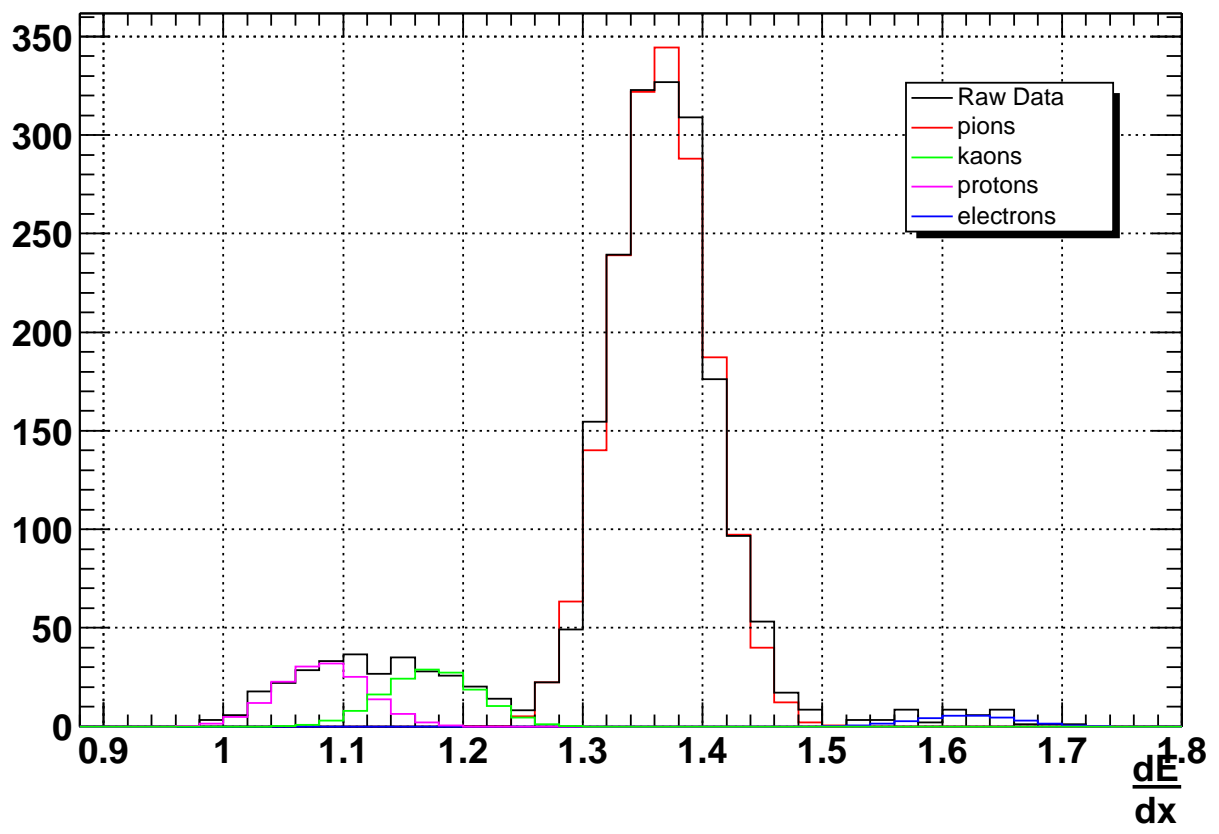

Figure 8.18: An example $\frac{d E}{d x}$ fit. 


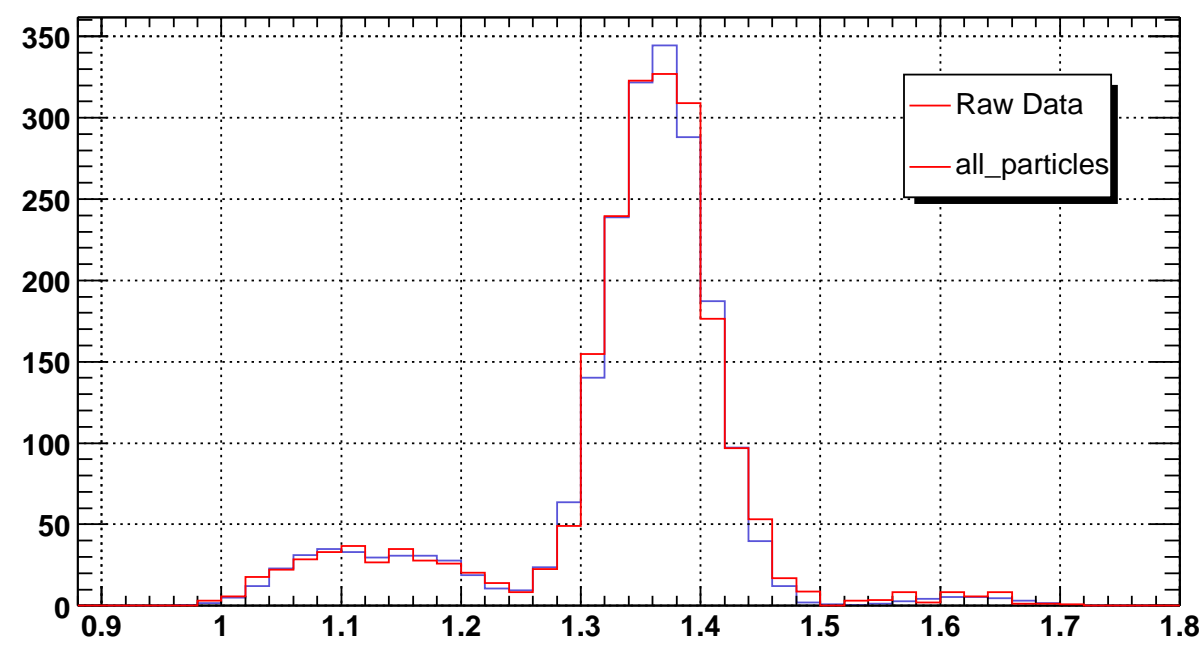

Figure 8.19: An example $\frac{d E}{d x}$ fit showing only the sum of the four particle fits. 


\section{Chapter 9}

\section{NA49 - Results}

Once the data had been through reconstruction, undergone calibration and sanitisation by the cuts described in the previous chapter, they were ready to be analysed and values to be calculated for the $K^{+} / \pi^{+}$ratio. There were two data runs taken by the NA49 experiment, one with a beam energy of $158 \mathrm{GeV}$ and one at $100 \mathrm{GeV}$. Unfortunately due to time constraints only about 700,000 events were taken at the lower energy, compared to about 2,300,000 events at $158 \mathrm{GeV}$. Therefore, due to low statistics, I have only been able to analyse the higher energy data set.

The NA49 group carry out all of their analysis in Feynman $x$ space $\left(x_{F}\right)$ rather than $p_{L}$ space. As I am using analysis techniques and software built on NA49 collaboration development I have followed the same approach. The Feynman $x$ variable, $x_{F}=\frac{p_{L}}{p_{L_{\max }}}$, is a scaling variable that was introduced by Feynman in 1969 [82] while discussing hadronic interactions at high energies. For binning of the data I used values of $x_{F}$ based on the maximum forward momentum of each particle assuming it was a pion. This was necessary as the identity of each particle is not known. Using Feynman $x$ instead of forward momentum allows direct comparison between different beam energies. 


\subsection{Binning}

As discussed in Chapter 7, the aim of the initial study was to provide values for the $K^{+} / \pi^{+}$ratio over a large area of the $p_{L}, p_{T}$ phase space. Figure 9.1 shows the distribution of events over this phase space.

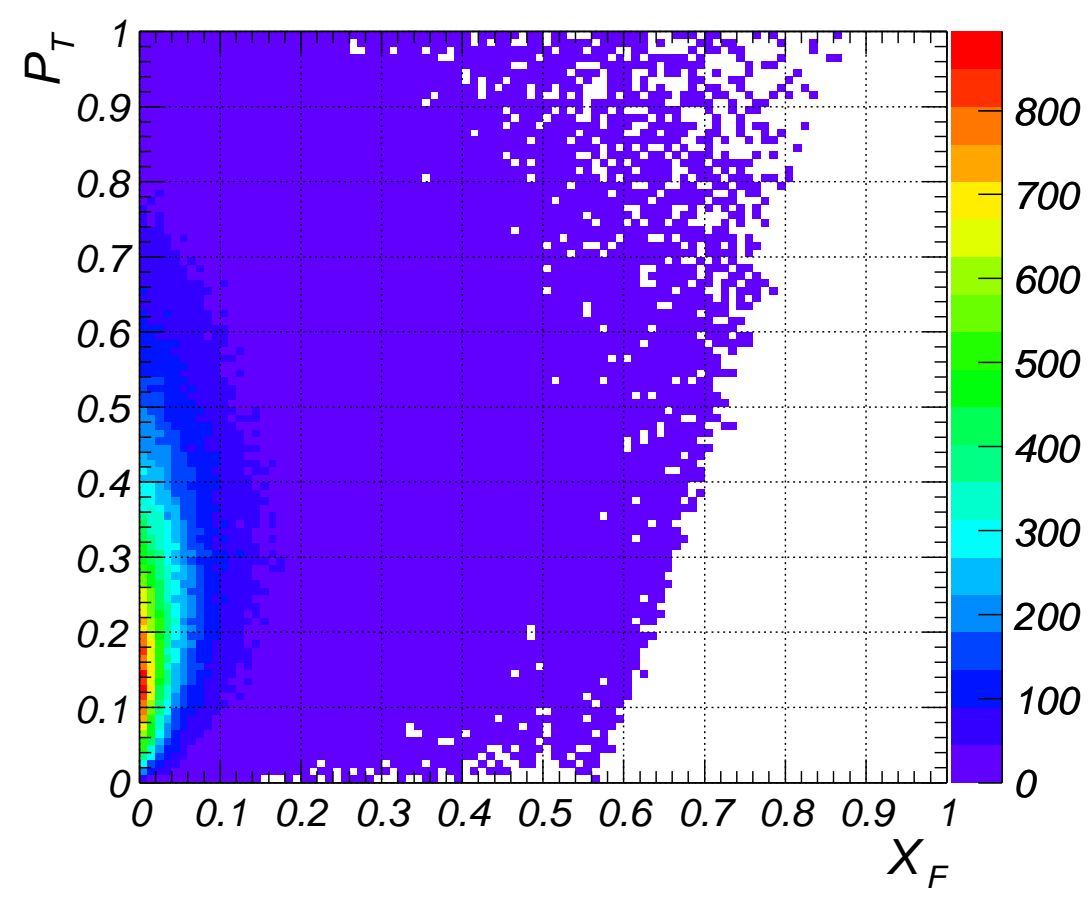

Figure 9.1: A plot showing the event distribution in $x_{F}$ and $p_{T}$ space. It can be seen that the acceptance of the detector is restricted at high values of momentum as expected. Also clear is the high distribution of events with $x_{F}$ below 0.1

The first task was to bin the data ready to be analysed. As figure 9.2 shows, the number of tracks decreases with $x_{F}$. Therefore, rather than create a fixed bin width, I decided to make the bin width variable. If a fixed bin width had been used then the size required to contain a satisfactory number of events at high $x_{F}$ would have been over wide at low $x_{F}$. So the resolution at low $x_{F}$ would not be maximised. 


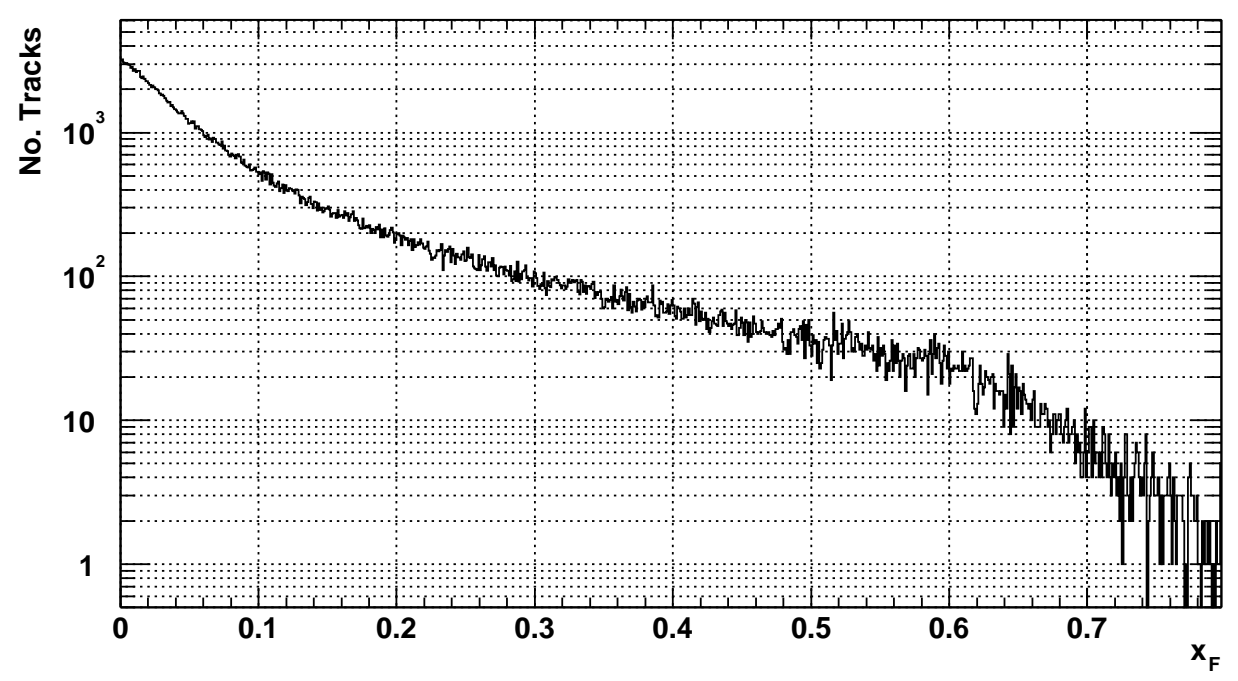

Figure 9.2: This plot shows how the number of tracks falls as $x_{F}$ increases. Therefore, for higher efficiency, variable bins widths are used.

Also it is important to try to make the spread in $x_{F}$ per bin as small as possible for other reasons than just statistical purposes. The narrower the bin width, the less spread in $\frac{d E}{d x}$ contained in the bin. This has the effect of reducing the width of the particle peaks enabling more accurate fitting. Figure 9.3 shows this in detail. With a large spread in the $\frac{d E}{d x}$ of the particles the peaks start to merge into each other, making fitting very difficult. This is especially important at low $x_{F}$ when the Bethe-Bloch curve is changing rapidly.

\subsubsection{Bin Representation Values}

Due to the distribution of particles in the momentum phase space, care has to be taken when defining the values of $p_{T}$ and $x_{F}$ representing each binned data set. This is because as the distribution is not flat, but declines with increased momentum in both directions, the mean value of the momentum represented is not the centre of the bin. Figure 9.4 shows this with some example plots. For this analysis I used the 

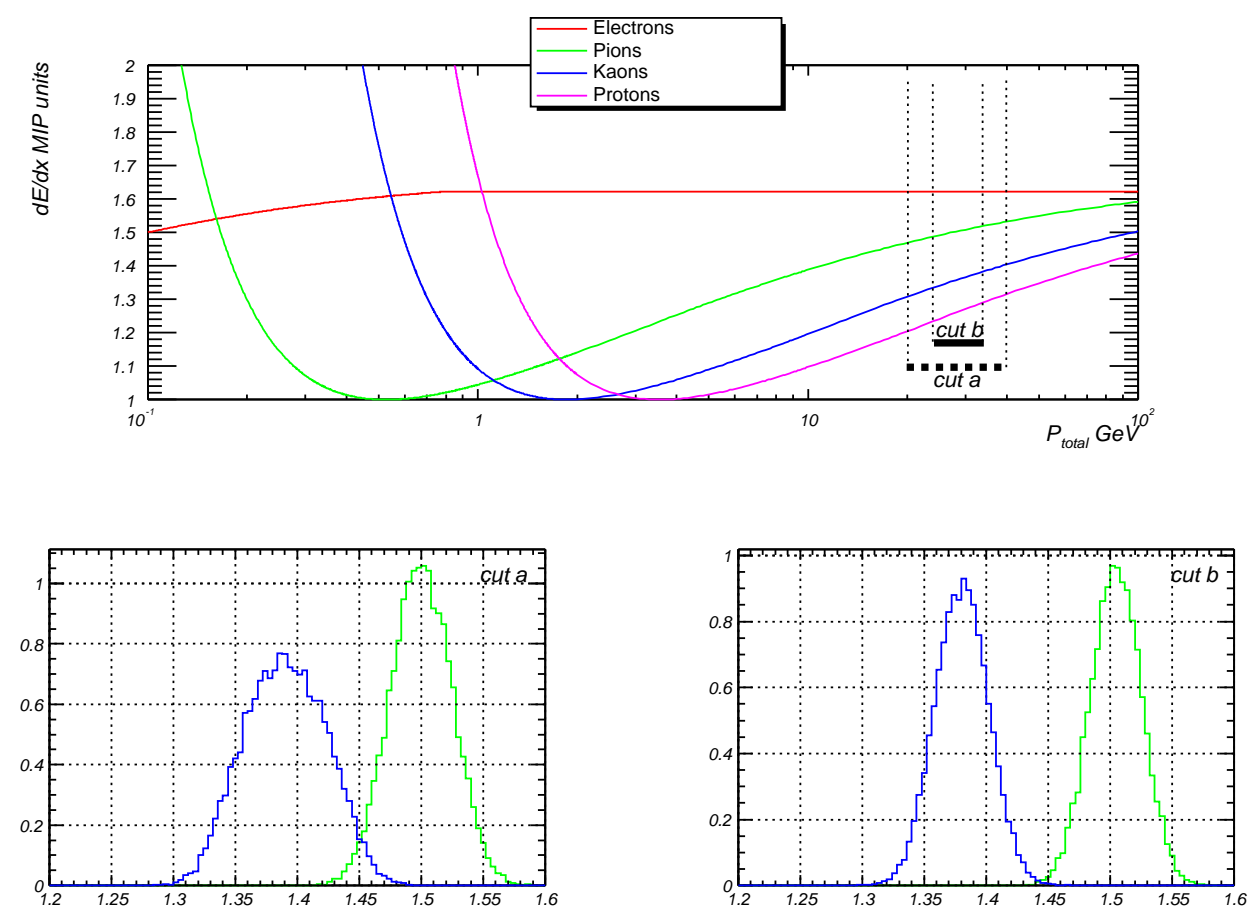

Figure 9.3: This schematic diagram demonstrates the need for the $x_{F}$ bins to be as small as possible. The top plot shows a portion of the Bethe-Bloch curve for all four particles. The two lower plots show projections of the portions of this curve, as shown by the dashed (cut a) and solid (cut b) lines, onto the y-axis. It is clear that on the right hand plot the distributions have a far clearer separation. 
mean of each binned data set.

\subsection{2 $p_{T}$ Constraints}

There were also upper and lower limits for which it would be possible to carry out the analysis in $p_{T}$ space. Various factors affected this. Firstly were the physical dimensions of the detector. At low momenta the momentum kick provided by the magnets would be too high compared to $p_{L}$. This would cause the particle to bend out of the TPC before enough data points were recorded. At the other end of the energy range, if the momentum of the particles were too high, then the kick provided by the magnets would not be enough to allow the particles to pass through the VTPCs. This would not enable the momentum of the particle to be calculated. For momentum calculation to be successful the curvature of the track needs to be measured. For this to happen the particle needs to pass through a TPC while a strong magnetic field is present. As the VTPCs are the only chambers within the fields, they must contain hits for the particle to be measured.

Figure 9.5 shows the different parts of the detector hit by particles of different $p_{L}$ and $p_{T}$. It can be seen that for particles below around $1 \mathrm{GeV}$ not all $p_{T}$ particles are contained within the detector. Similarly, for particles with $p_{L}$ above around 40 $\mathrm{GeV}$, the lower $p_{T}$ particles do not have enough curvature to be counted within the VTPCs.

The affect of this acceptance can be seen in figure 9.1, where at high values of forward momentum, $x_{F}$, only particles with higher transverse momentum are accepted. These are the only particles that pass through a vertex TPC and leave enough track points to enable a fit. 

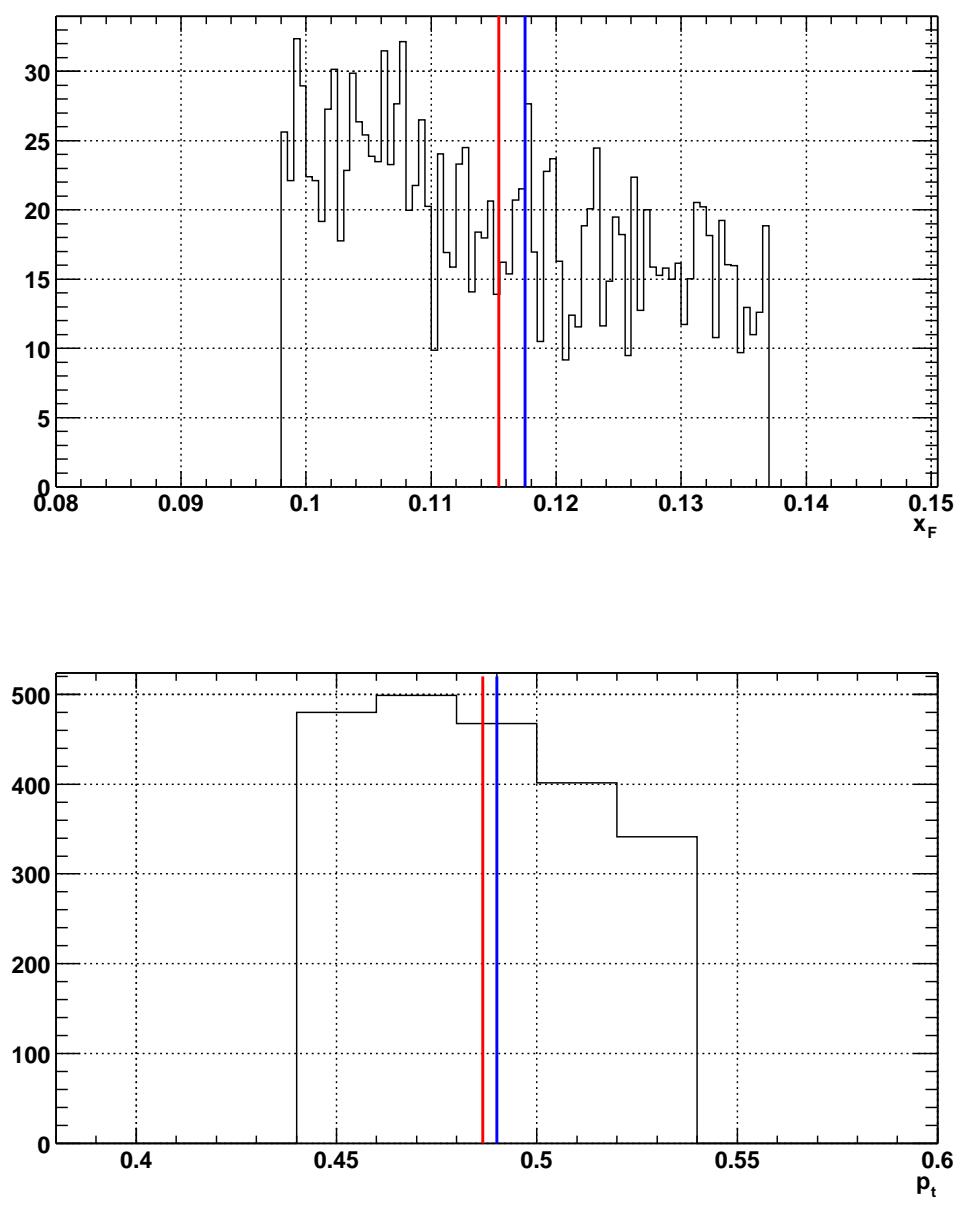

Figure 9.4: These two plots show the need to take into account the non-flat distribution of particles as momentum increases, both longitudinal (represented by $x_{F}$ ), and transverse $\left(p_{T}\right)$. In both plots the blue line represents the middle of the bin, while the red shows the mean value for the histogram. It can be seen for both that there is a distinct difference between the two values. 


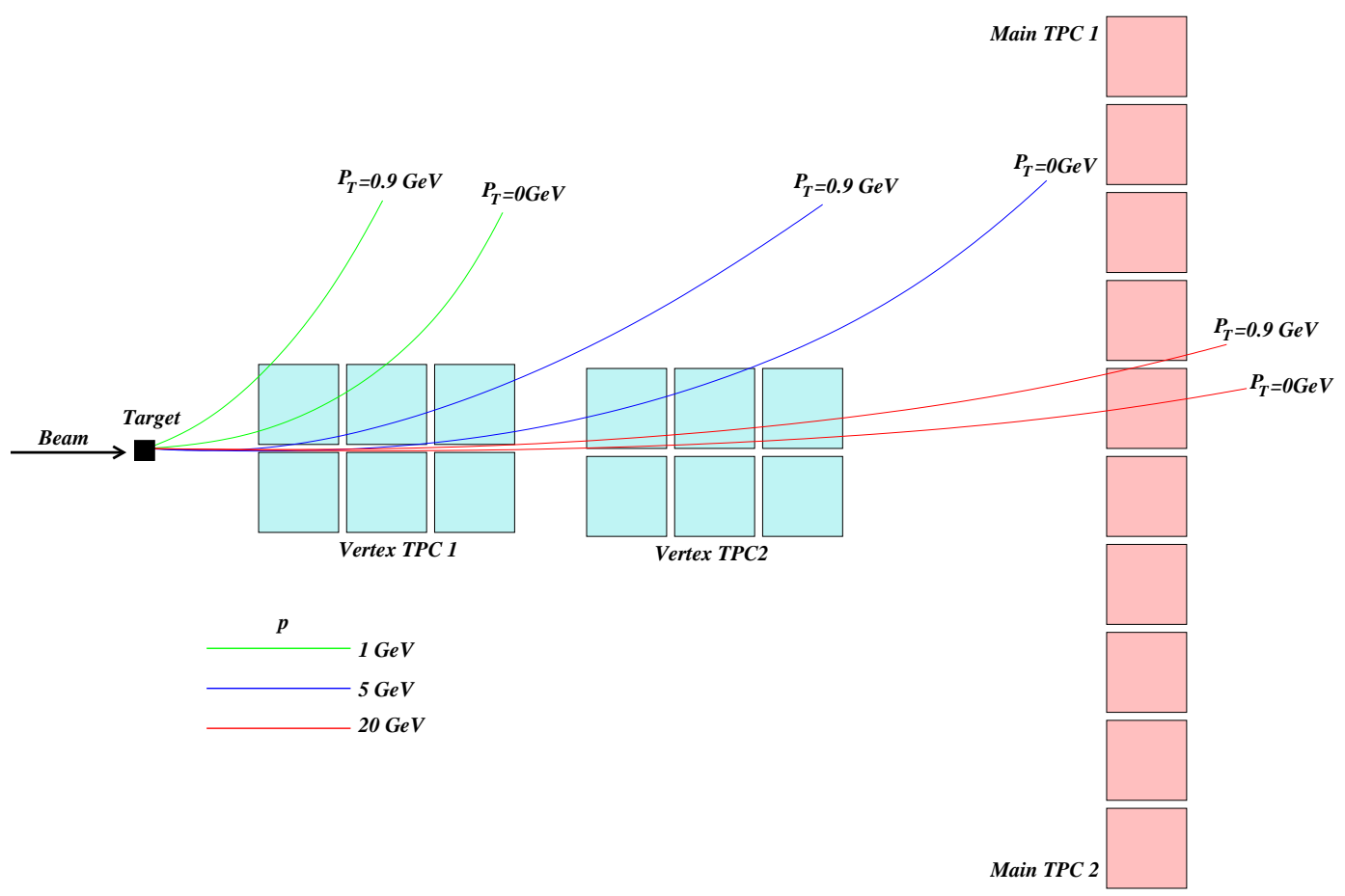

Figure 9.5: Schematic showing the acceptance of the NA49 for several positive charged particles with differing values of $p$ and $p_{T}$. Because of this acceptance issue, only momentum ranges with $100 \%$ acceptance were kept. 


\subsection{Results}

I analysed the data from two different perspectives, firstly I looked for a relation between the $\frac{K^{+}}{\pi^{+}}$ratio and $x_{F}$; I then looked at the ratio with respect to $p_{T}$.

\subsection{1 $p_{T}$ Slices}

Plot 9.6 shows the method I used to bin the data. Firstly I divided the data into bins in $p_{T}$ phase space, requiring each bin to contain 20,000 events. These can be seen as the horizontal lines in figure 9.6. I then subdivided each of these large bins into 10 smaller bins, each with 2,000 entries. It can be seen from this diagram that the bins with high $x_{F}$ begin to cover a region of space with limited acceptance, the bins are also unacceptably large. Therefore I have only fitted those bins which fall inside the acceptance of the detector.

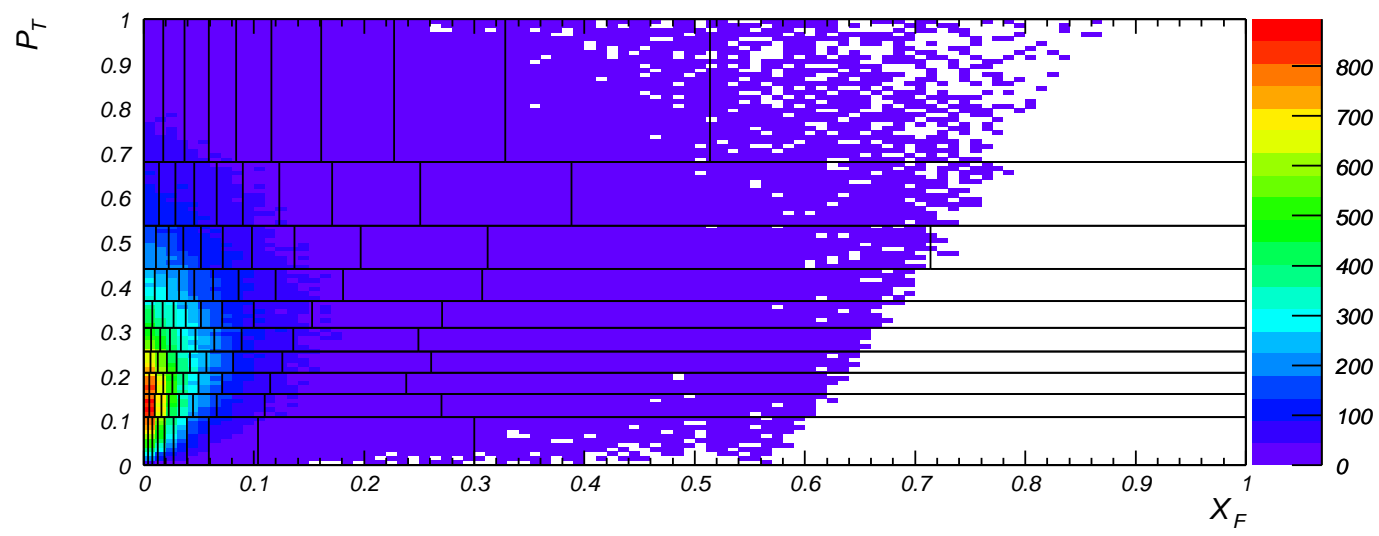

Figure 9.6: This plot shows the binning made based on $p_{T}$.

After the data was binned it was fitted using the methods previously described.

Figures 9.7 and 9.8 show the yields of the pions and kaons calculated from the fitting routines. Ideally one would plot the absolute number of each particle detected. However, the total number of particles in each bin includes protons and 
electrons, making this impossible. Therefore the yields are shown as a percentage of the total number of pions and kaons in the bin.

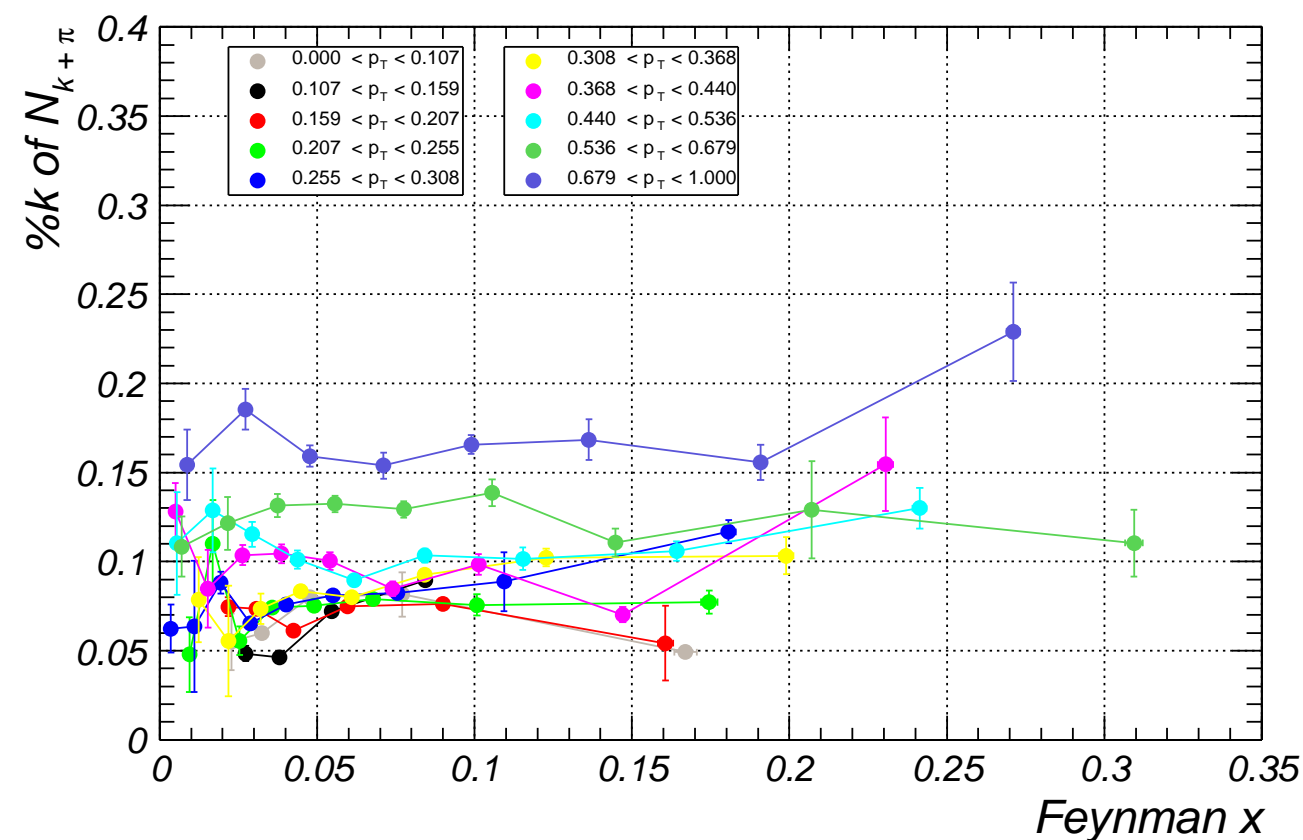

Figure 9.7: Kaon production (as a percentage of total kaon and pion production) as a function of $x_{F}$.

These two yields combined provide the $\frac{K^{+}}{\pi^{+}}$ratio, as shown in figure 9.9. This shows that there is not a clear dependence of the $\frac{K^{+}}{\pi^{+}}$ratio with $x_{F}$, however there is a clear dependence on $p_{T}$, the higher $p_{T}$ bins have a higher $\frac{K^{+}}{\pi^{+}}$ratio.

\subsection{2 $x_{F}$ Slices}

The data were then binned again in a similar fashion to the previous section. This time the initial binning was in $x_{F}$. Once again the data were divided into bins with 20,000 entries, these bins were then subdivided into bins with 2,000 events in $p_{T}$ as shown in figure 9.10. The same considerations for detector acceptance were taken 


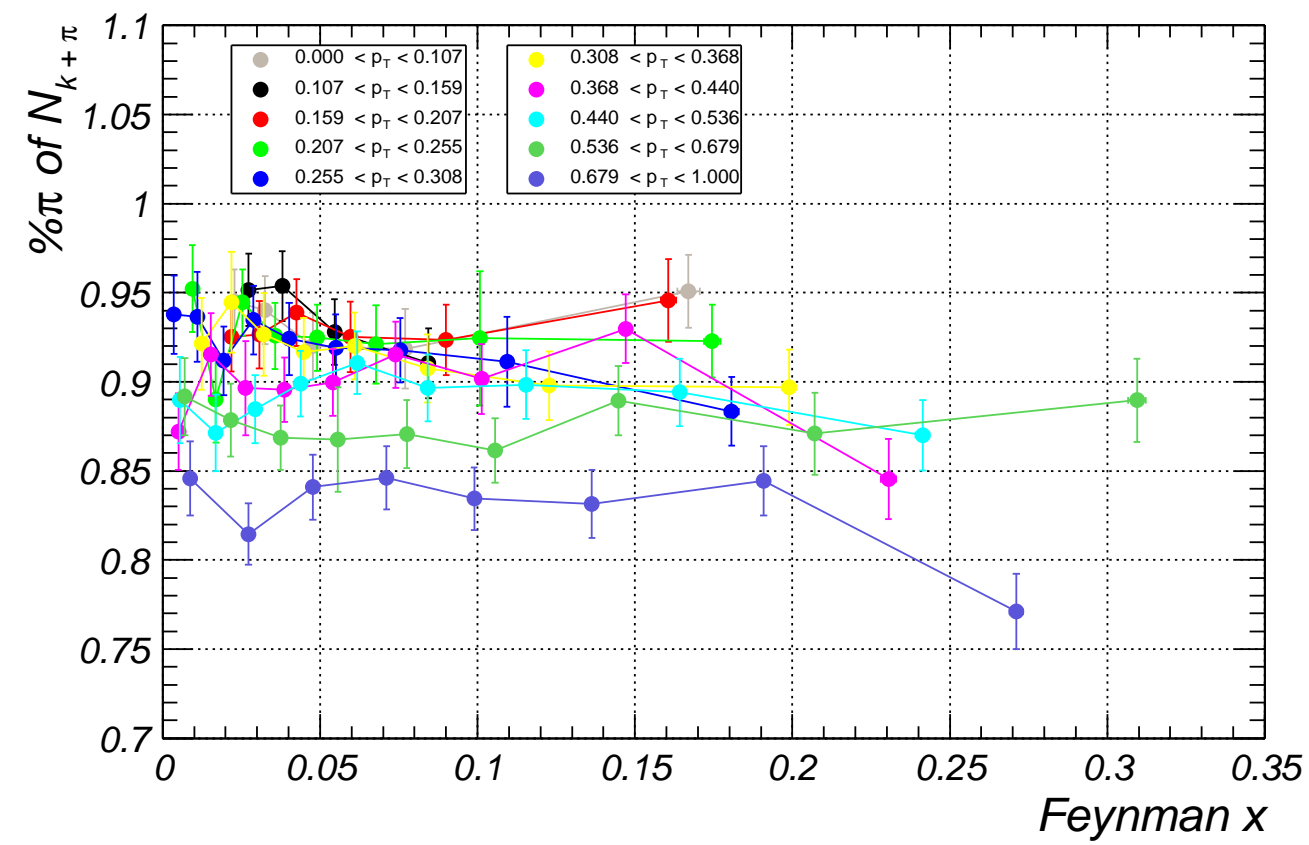

Figure 9.8: Pion production (as a percentage of total kaon and pion production) as a function of $x_{F}$. 


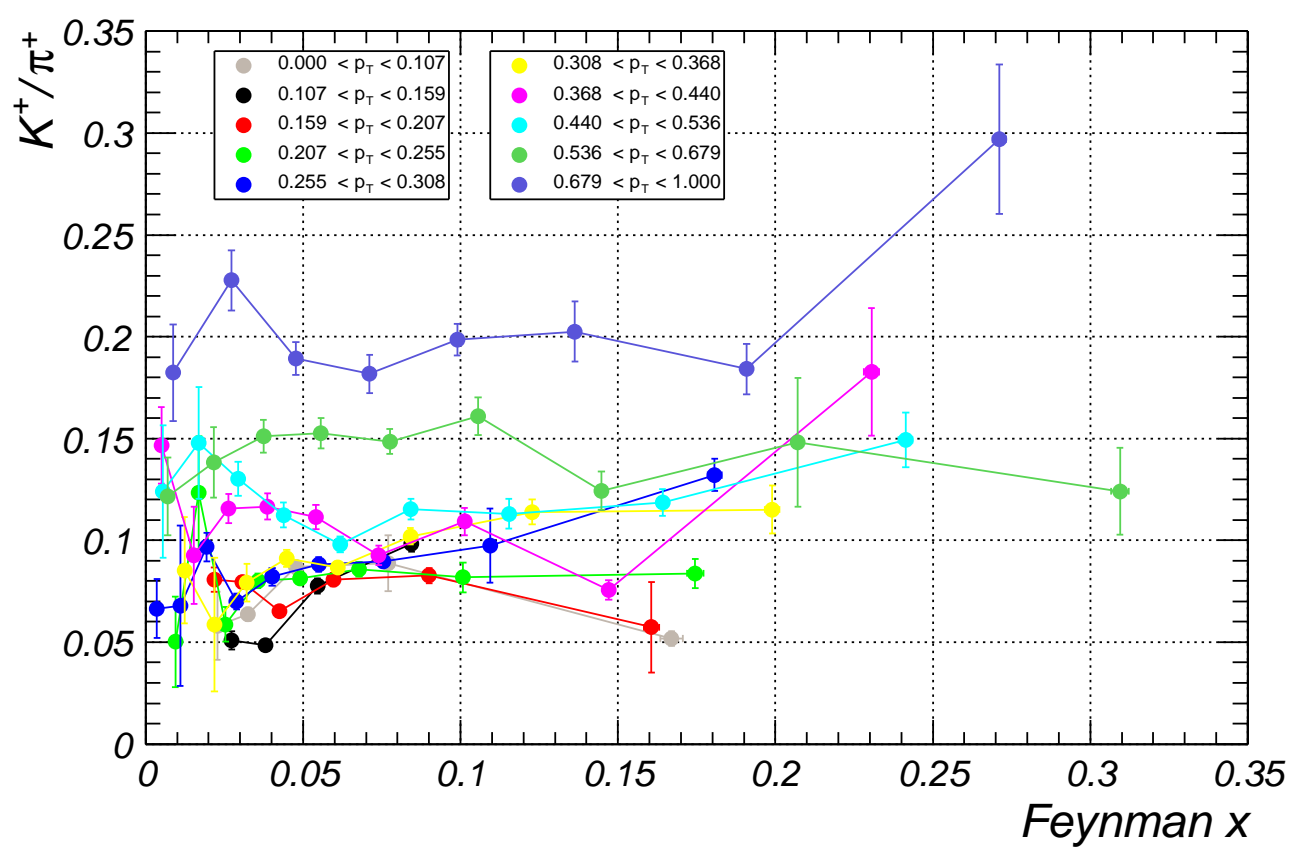

Figure 9.9: $\frac{K^{+}}{\pi^{+}}$as a function of $x_{F}$. 
as in previous analysis, with only those bins with $100 \%$ acceptance being acepted.

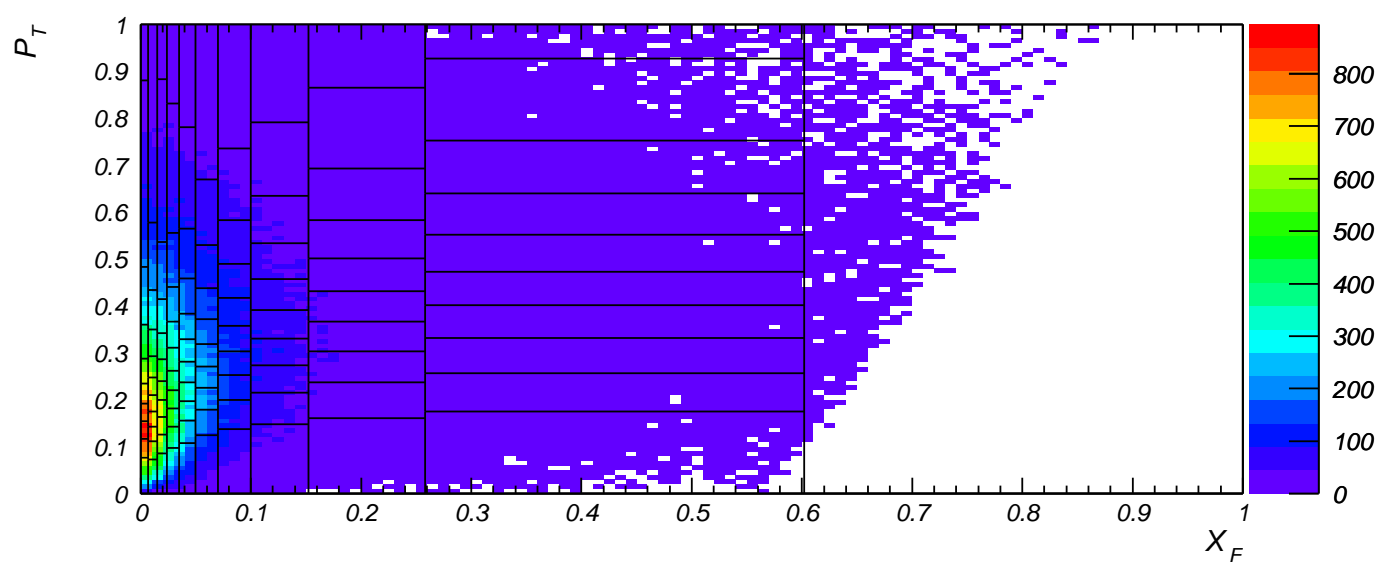

Figure 9.10: This plot shows the binning made based on $x_{F}$.

Once again the individual yields for the kaons and pions is shown as a percentage of the total number of kaons and pions in figures 9.11 and 9.12.

These yields combined provide the $\frac{K^{+}}{\pi^{+}}$ratio, shown in figure 9.13. A clear correlation is seen here between transverse momentum and the $\frac{K^{+}}{\pi^{+}}$ratio. 


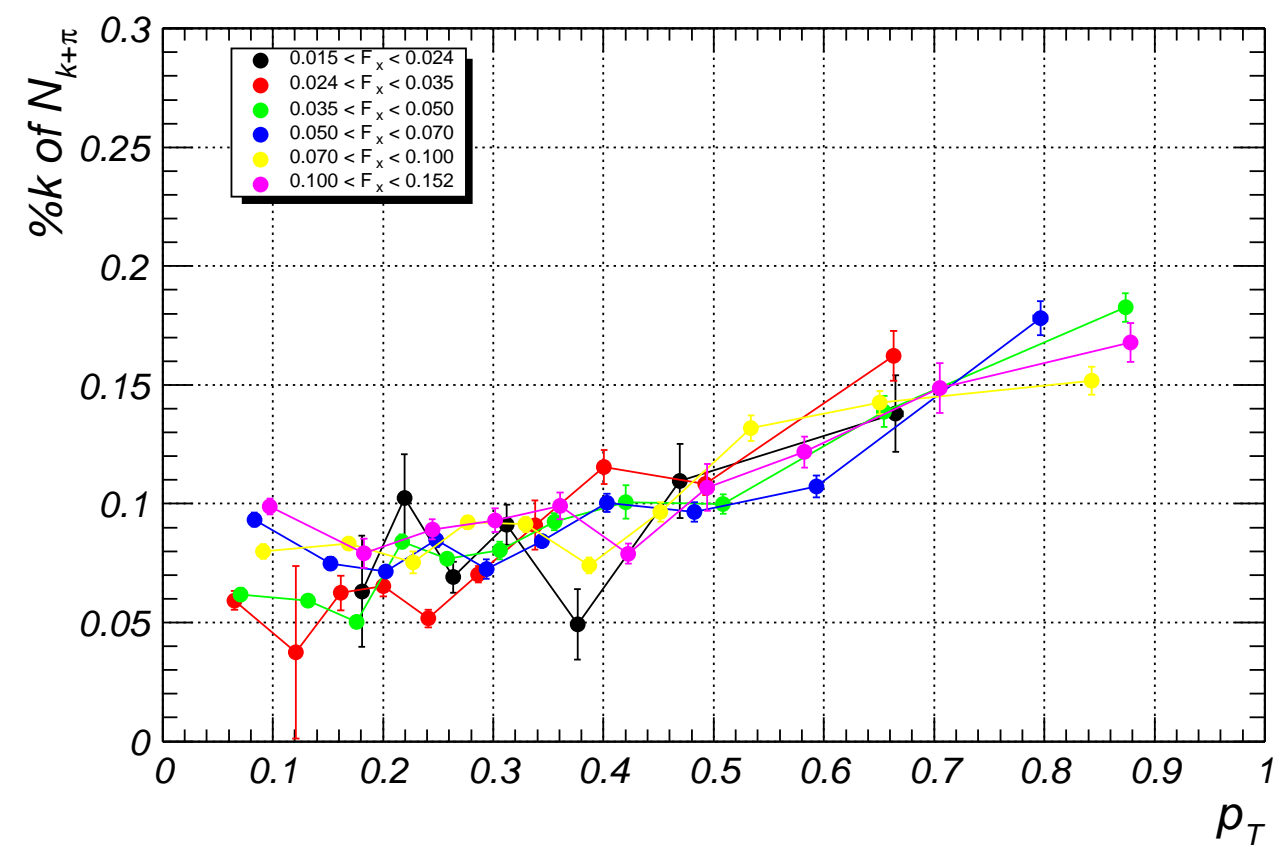

Figure 9.11: Kaon production (as a percentage of total kaon and pion production) as a function of $p_{T}$. 


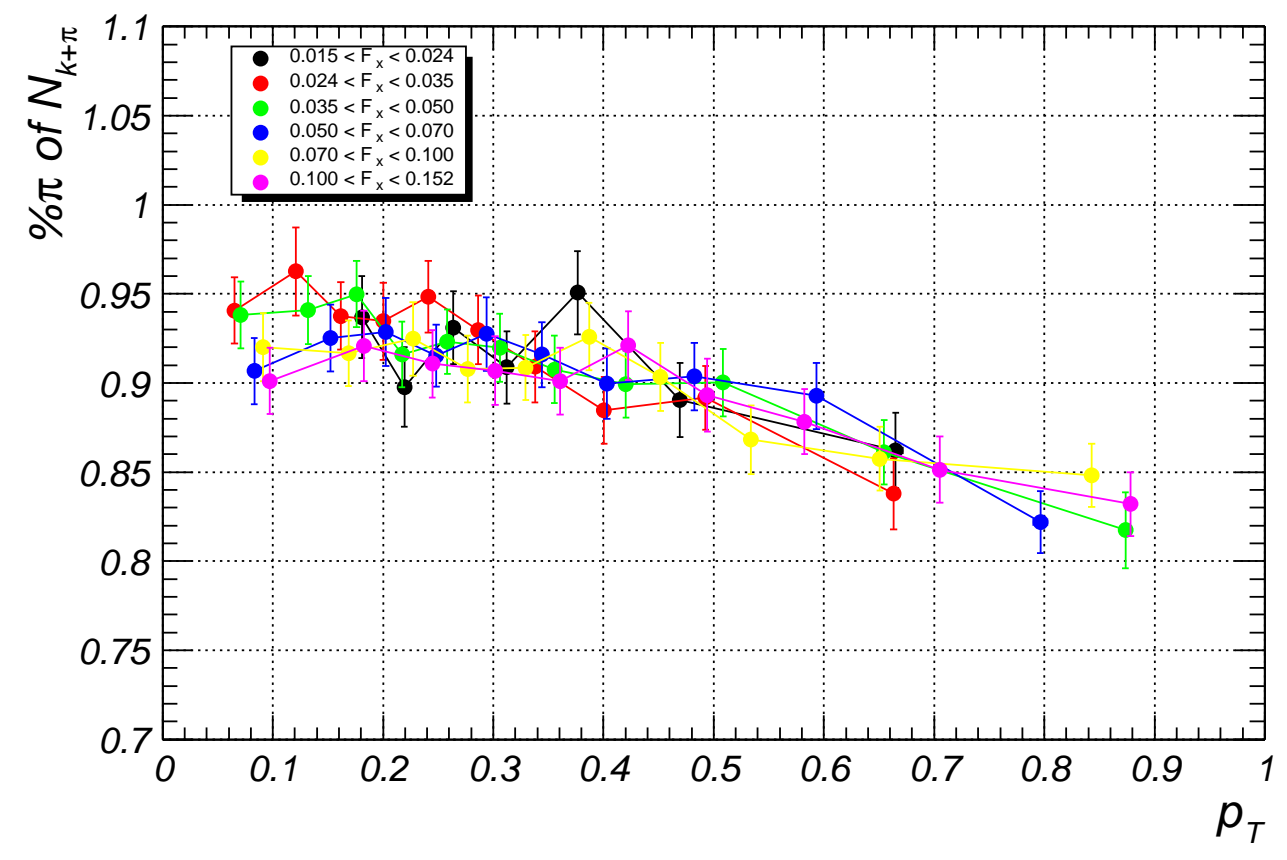

Figure 9.12: Pion production (as a percentage of total kaon and pion production) as a function of $p_{T}$. 


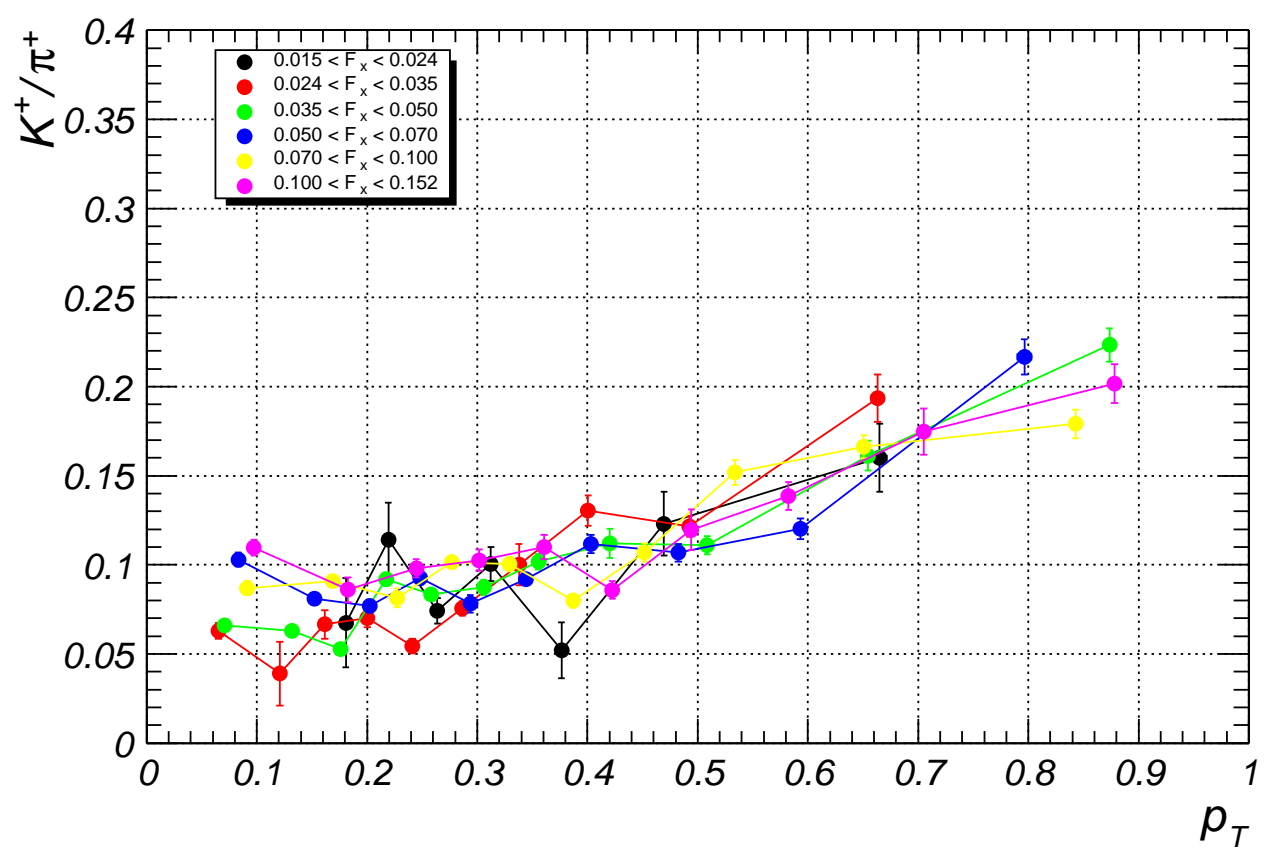

Figure 9.13: $\frac{K^{+}}{\pi^{+}}$as a function of $p_{T}$. 


\subsection{Comparison with Previous Experiments}

A direct comparison between the data presented above and the results obtained from previous hadronic production experiments is difficult. Most other experiments such as Barton [72] and Atherton [73] focused at much higher momenta, often with zero $p_{T}$. The experiment with the closest energy ranges to the NA49 $\mathrm{p}-\mathrm{C}$ run was the SPY [74] experiment. Figure 9.14 shows the results from the SPY experiment overlaid on the relevant NA49 data.

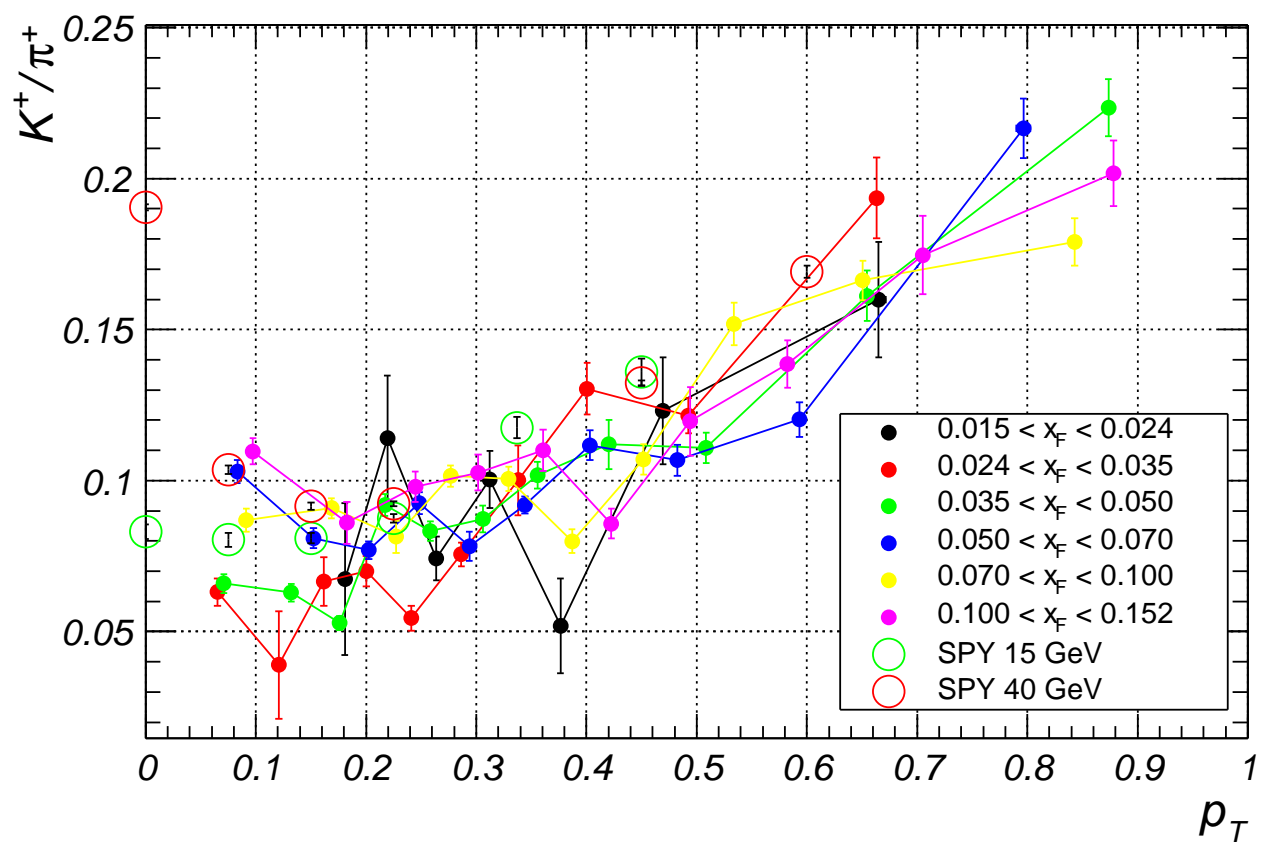

Figure 9.14: Comparison of NA49 hadronic production data with SPY 15 and 40 $\mathrm{GeV}$ data.

Figure 9.15 shows a more detailed comparison between the SPY $15 \mathrm{GeV}$ data [74] and the relevant binned data from the NA49 p-C run. 


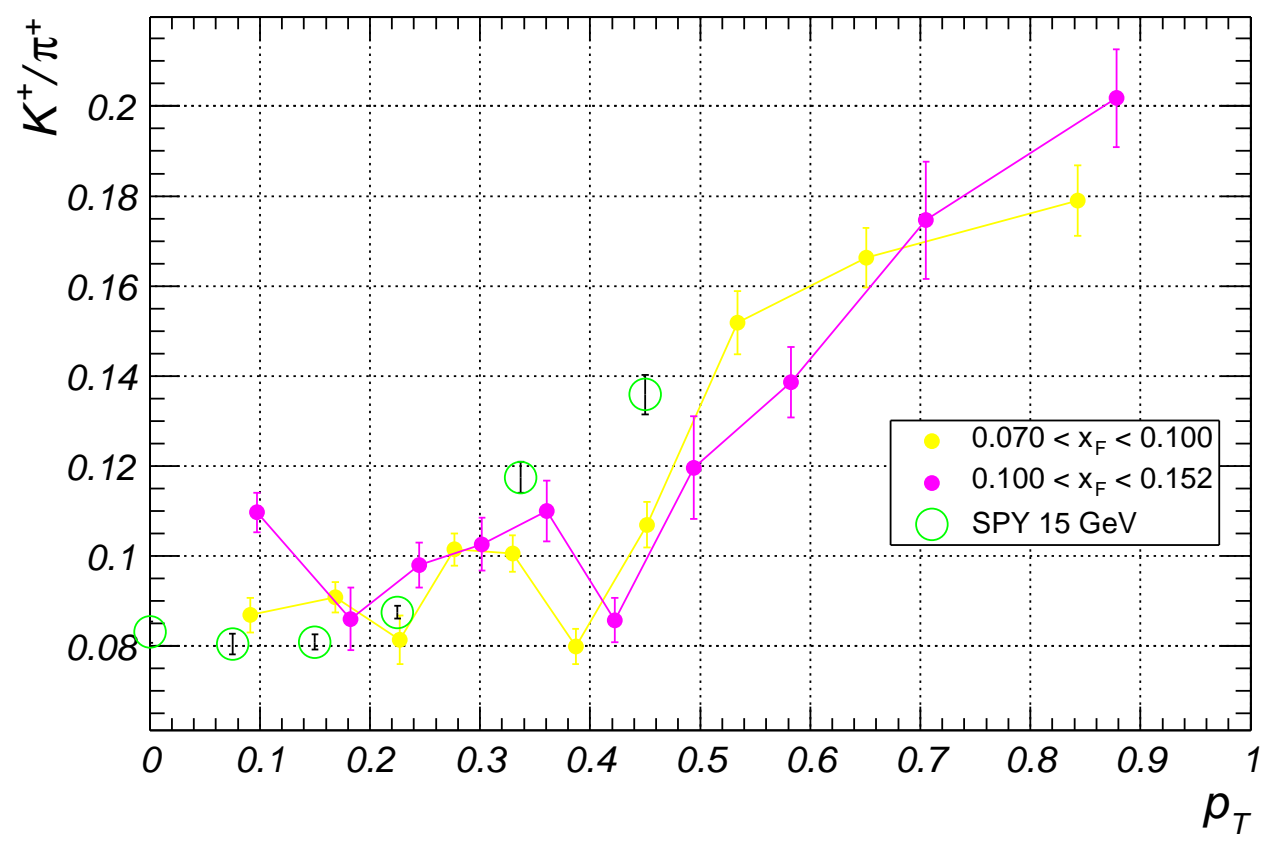

Figure 9.15: Comparison of NA49 hadronic production data with SPY $15 \mathrm{GeV}$ data. The two data sets from the NA49 run have comparable momentum to that of the SPY data. 


\section{Chapter 10}

\section{Conclusions}

At the time of writing, construction of the MINOS far detector was nearing completion. With the second supermodule complete and the magnetising coils in place. The calibration detector is in the final stages of its life taking data at CERN, characterising electronics to be used at the near detector. Construction of the near detector itself is well underway, and running according to schedule. The NuMI beam line is scheduled to be complete by the end of 2004, providing MINOS with its first measurable neutrino spectrum.

Within two years of running, MINOS will have confirmed neutrino oscillations as the source of the atmospheric neutrino anomaly. MINOS will make the first precision measurements of the $\nu_{\mu} \rightarrow \nu_{\tau}$ mixing parameters: It will measure the mixing parameters $\sin ^{2} 2 \theta_{23}$ and $\Delta \mathrm{m}_{23}^{2}$ to within $10 \%$. It will also place tighter constraints on $\theta_{13}$ by searching for electron neutrino appearance. This will be important in determining the final designs of the off-axis experiments that are now under development which take advantage of the spread of the numi beam.

The light injection system was delivered to specification, on time, and $40 \%$ below budget. It has enormous flexibility, and is very robust; and with no moving 
parts, it is expected to be maintenance-free throughout the lifetime of MINOS. It has clearly demonstrated that it is able to monitor and to compensate for gain changes at the $1 \%$ level, as required. It is also being used now to establish the gain curves of all 200,000 MINOS readout channels, thus characterising the PMT responses from single-photoelectron levels up to complete saturation. It has already been used extensively as a very powerful test and development tool within MINOS. It pushes the detector, and the data acquisition system, to its limits, and several potential problems have been discovered and solved as a result.

The proton-carbon data showed a strong increase of the $\frac{K^{+}}{\pi^{+}}$ratio with $p_{T}$, with little apparent dependence on $x_{F}$. This greatly extends the range of existing data as shown at the end of chapter 9. In conjunction with the MIPP results that are expected to be forthcoming within the next few years, these results will provide valuable reference data for the development of Monte Carlos to predict the nature of the NuMI beam at the MINOS Near and Far Detectors. In addition, these data are already being incorporated into new models of atmospheric neutrino production. 


\section{Appendix A}

\section{NA49 $\frac{d E}{d x}$ Fitting Parameter Checking}

During the fitting of the binned $\frac{d E}{d x}$ data, some of the fitting parameters were edited by hand. Due to this a great deal of caution had to be used to prevent the fitting being swayed by human intervention. To check this, I investigated any trends behind the modifications used to shift the mean of each particles distributions from the expected Bethe-Bloch curve. Figures A.1 and A.2 show an indication of the changes in these fitting parameters.

Any non-physical solutions found by manipulating the fitting parameters should expose themselves here as drastic changes. It can be seen however that all the parameters in all situations are slowly varying and move together, implying that they represent real variations from the expected values. 

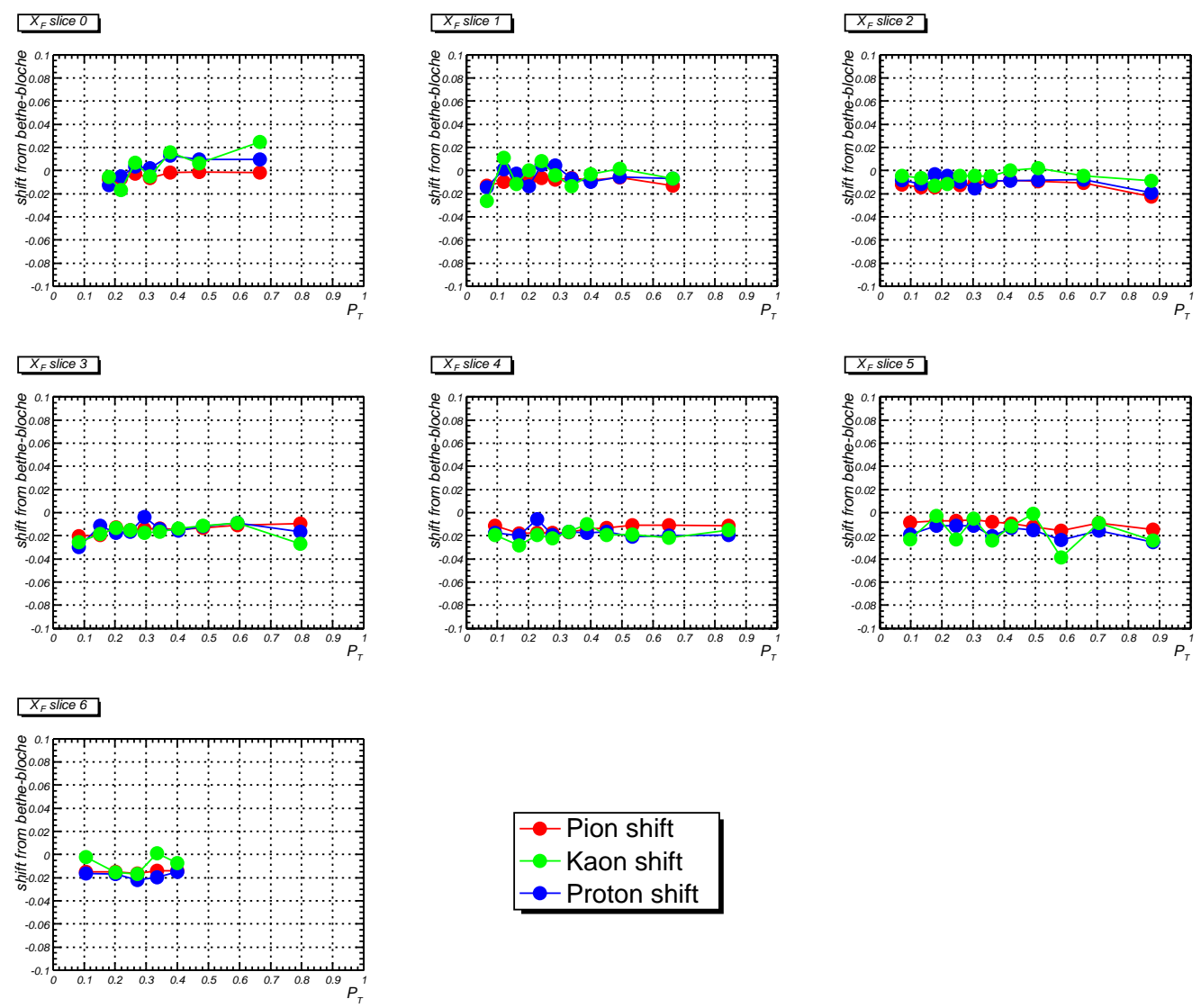

Figure A.1: Shown here are the shifts from the theoretical Bethe-Bloch means used for the fits of the $x_{F}$ slices of the data. 

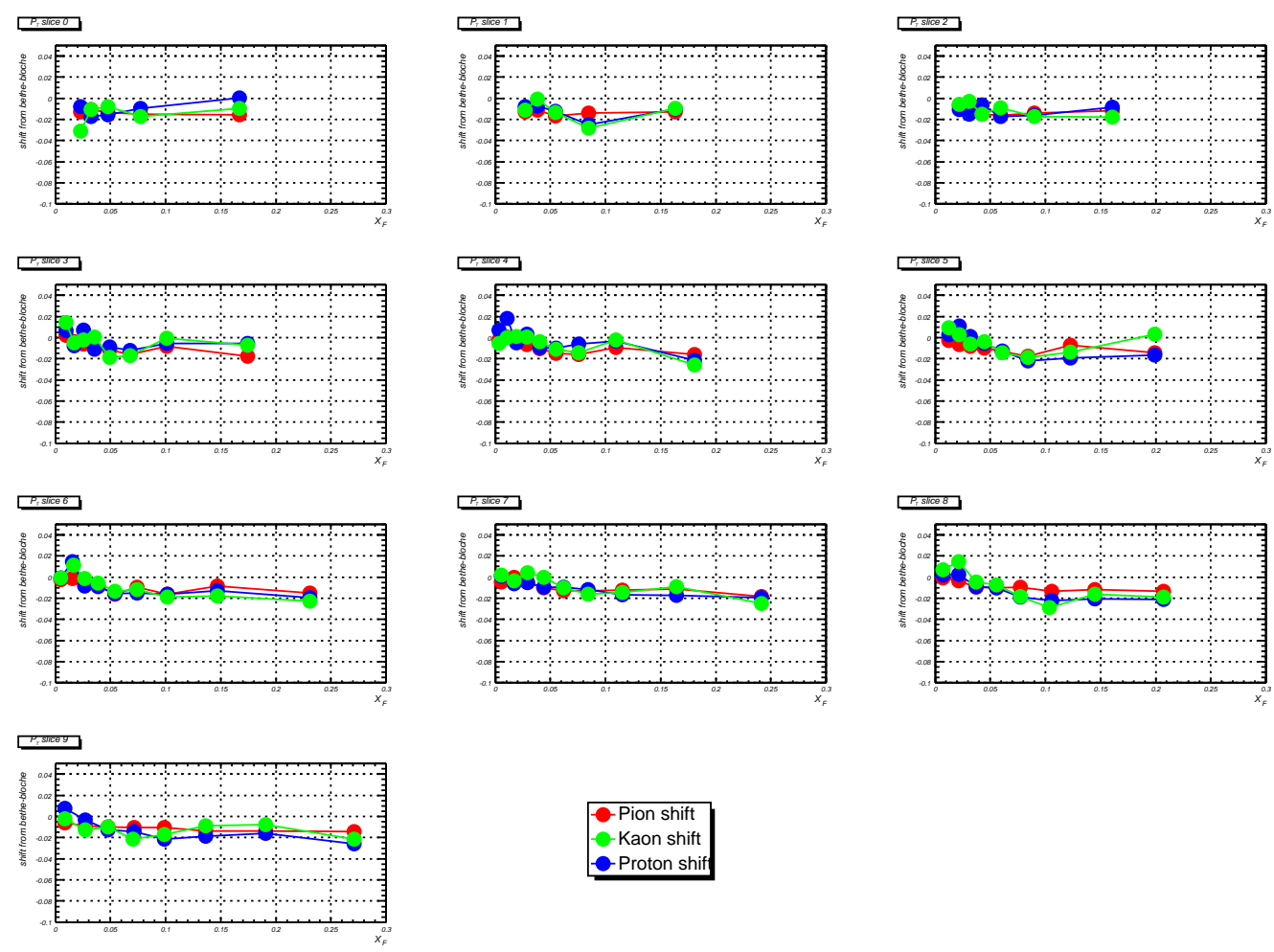

-Proton shif

Figure A.2: Shown here are the shifts from the theoretical Bethe-Bloch means used for the fits of the $p_{T}$ slices of the data. 


\section{Appendix B}

\section{MINOS Light Injection Software}

During my time working on the MINOS light injection system I was deeply involved in the installation and debugging of the detectors. To help with this I developed many pieces of software ranging from on the spot scripts to deal with day to day issues to more in depth programs consisting of many thousands lines of code. Detailed below are just two of the more useful and persistent pieces of software developed.

\section{- LI Summary}

Due to the extremely high data rates from the MINOS detectors from the thousands of channels, a complex software framework was developed to manage and analyse the data. This framework was based upon ROOT a C++ software environment developed by CERN especially for the particle physics community. The LI summary package that I wrote was designed to identify and extract the data relevant to the light injection system calibration runs. The data was then analysed to extract the various calibration parameters needed, such as PMT gain drifts.

- Gryfindor 
This piece of software was developed to monitor the installation of the light injection system at the far detector. Due to the multiplexing of the readout fibres to the PMTs, the pattern of pixels pulsed on each tube was not intuitive. Figures B.1 and B.2 show screen shots of the software in operation. It has the ability to read in MINOS data files, extract and decode the light injection data contain within, then plot the resulting pulse heights seen by each pixel on a PMT's face. By simultaneously showing the expected pulsing pattern a visual check can then simply be made to ensure that the right pixels are being pulsed. If there is a discrepancy, it is possible to change the pattern of expected pulses to investigate where the mis-wiring has taken place. This removes the need to check each wire physically and therefore saves a great deal of time. 


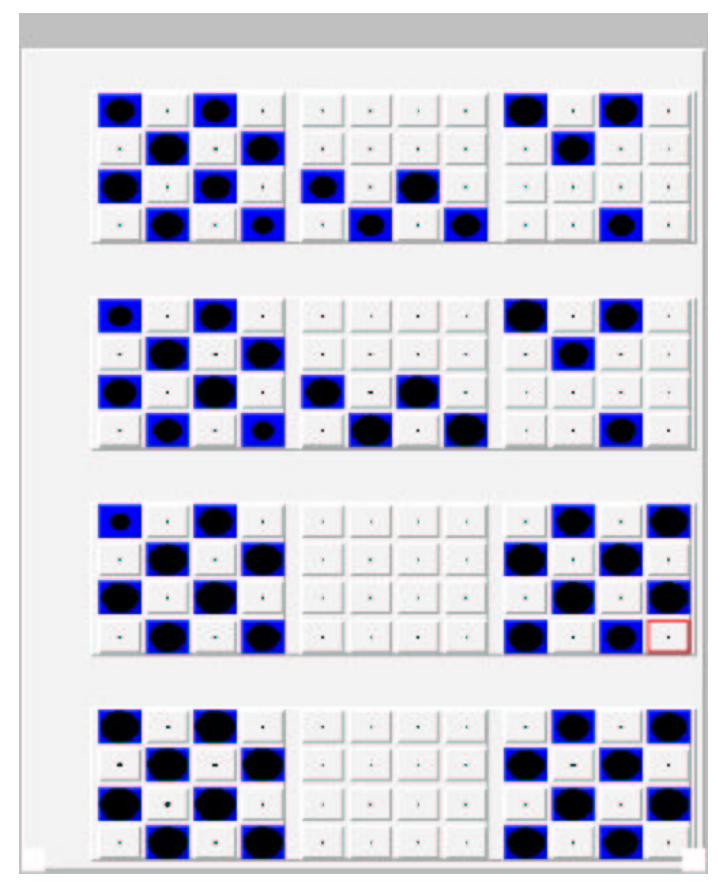

Figure B.1: A screenshot of the Gryfindor software used to check the integrity of the light injection system. Each set of 16 squares represents a PMT, and each set of three PMTs are grouped in MUX boxes. By comparing the received light (black circles, size proportional to signal size) with the expected illuminated pixels (blue squares) the pulsed patterns can be checked. It can be seen that this figure represents a correctly wired system. (The red square is an artifact of the drawing package). 


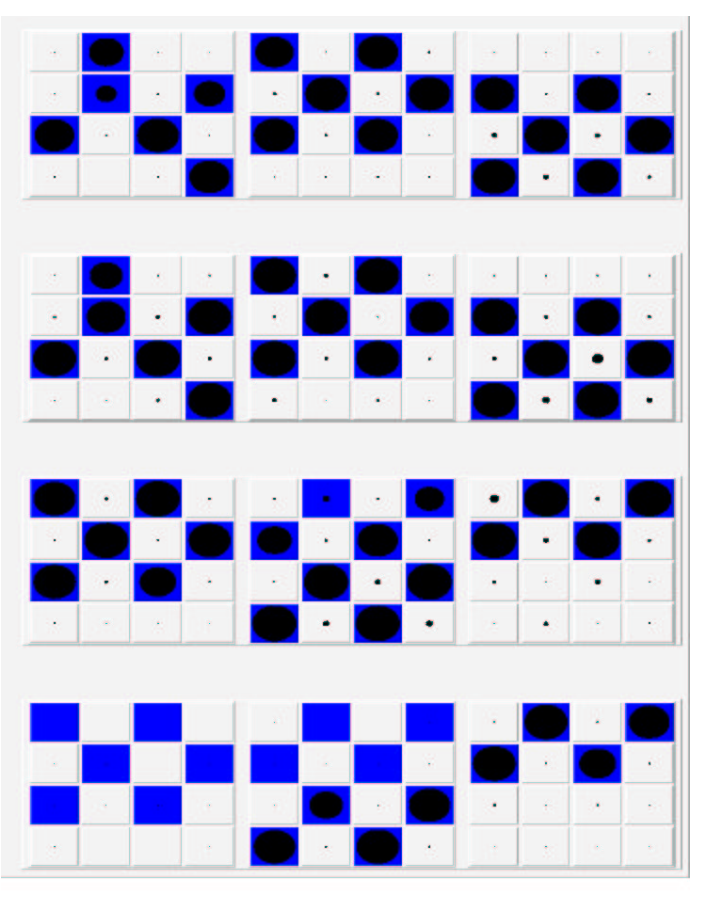

Figure B.2: This example of the Gryfindor software shows a situation where a cable has broken. In the bottom left and middle PMTs blue squares are indicating there should be pulses received; however no light is visible. 


\section{Appendix C}

\section{NA49 Software Development}

As shown in chapter 7, the fitting method involved in the $\frac{d E}{d x}$ particle yield analysis is very time consuming. It is also a very difficult method to employ, as human intervention is needed to prevent the fit converging on a non-physical situation. To make this process quicker I wrote a graphical user interface (GUI) front-end to enable quick manipulation of the fitting parameters. Figure C. 1 shows a screen shot of this program running on an example data set. The main left hand panel shows a histogram of the mean $\frac{d E}{d x}$ values for all the particles in the data set. Overlaid in this plot are the current distributions of pions, kaons, electrons and protons produced by the fitting software.

The purpose of this plot is to check for non-physical solutions. Due to the method of varying the means of the particle distributions it is possible for the fitting method to veer towards a non-physical solution. These are obvious to note, the fitting routine suddenly shifts one distribution under another. Therefore by monitoring this plot, these situations can be identified. In some cases it is impossible to converge on a real solution, if this happens the data bin has to be set aside as impossible to fit with this method. 


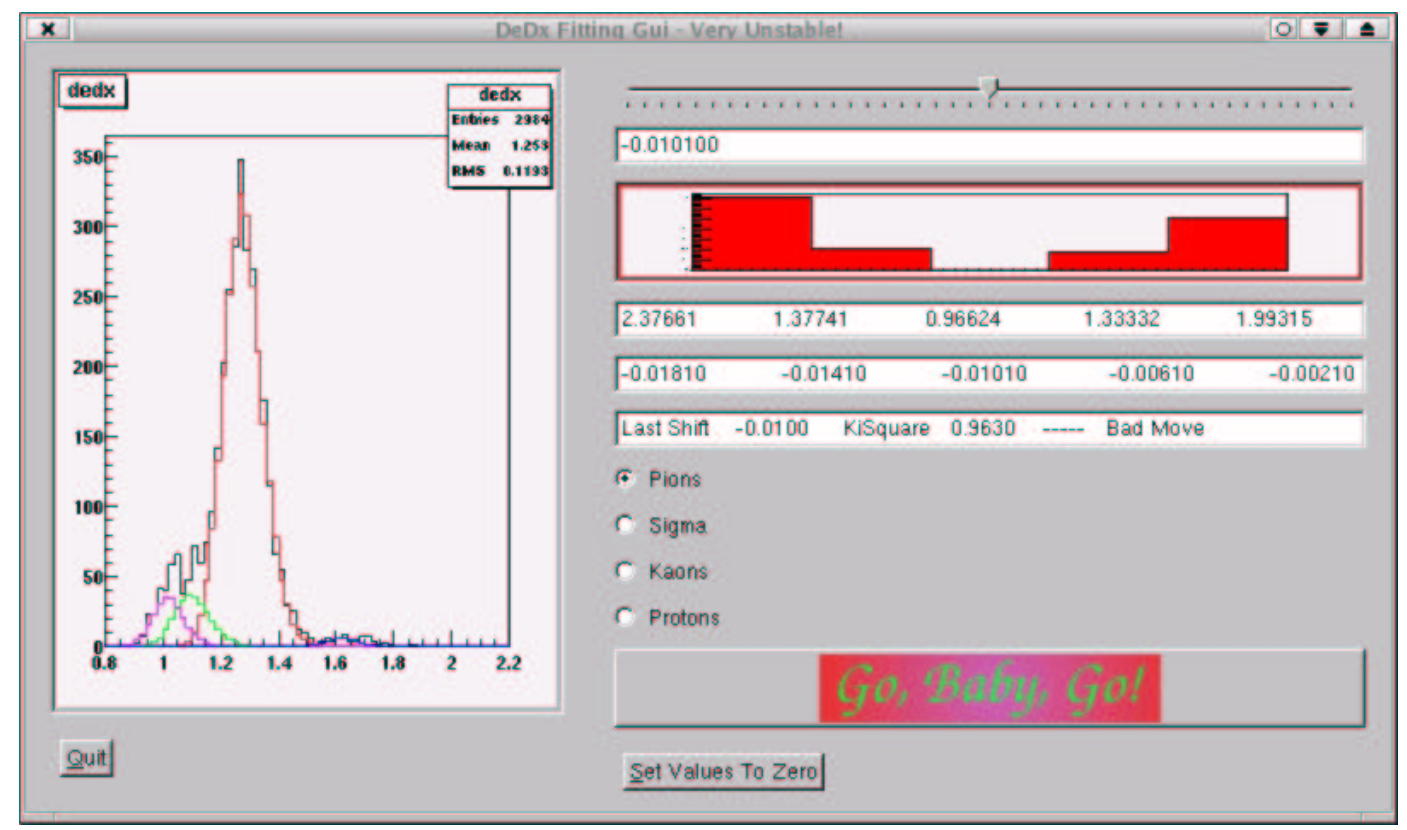

Figure C.1: Screenshot of NA49 analysis package

The second histogram panel, in red, is used to look at the quality of the fit according to the selected parameter. For example, in this situation the parameter governing the mean of the pion distribution has been altered. This histogram shows the goodness of the fit for the parameter at this value, as well as the improvement/deterioration possible by altering the parameter to two values either side of the selected value.

The text fields on the right side of the GUI show the numerical information implied by the parameter slider at the top of the screen, and the resulting fit goodness shown by the quality of fit histogram. The bottom text box shows the previous result from the fit, enabling the user to tell whether the change in parameters has caused an improvement in the fit, or has been detrimental.

This software was very beneficial in analysing the NA49 data, and extracting the particle yields from $\frac{d E}{d x}$ measurements. The time taken to analyse each data set was reduced by a factor of 10 . It was this that allowed the data to be analysed and 
fitted in such a large number of bins. 


\section{Bibliography}

[1] P. Adamson et al., Nucl. Instrum. Meth. A492, 325 (2002).

[2] P. Adamson et al., to be submitted to NIM. .

[3] G. J. Neary, Proc. Phys. Soc.(London) A175, 71 (1940).

[4] W. Pauli, letter, reproduced in translation in September 1978 Physics Today.

[5] C. L. Cowan, Jr. et al., Science 124, 103 (1956).

[6] C. Wu et al., Phys. Rev. 105, 1671 (1957).

[7] M. Goldhaber, L. Grodzins, and A. Sunyar, Phys. Rev. 109, 1015 (1958).

[8] S. Abrams et al., Phys. Rev. Lett. 63, 2173 (1989).

[9] B. Adeva et al., Z. Phys. C51, 179 (1991).

[10] and G. Alexander et al., Z. Phys. C42, 175 (1991).

[11] P. Abreu et al., Nucl. Phys. B367, 511 (1991).

[12] D. Decamp et al., Z. Phys. C53, 1 (1992).

[13] D. Decamp et al., Phys. Lett. 276B, 247 (1991).

[14] R. Davis, Jr., D. S. Harmer, and K. C. Hoffmann, Phys. Rev. Lett. 20, 1205 (1968).

[15] B. Pontecorvo, JETP 33, 549 (1957). 
[16] B. Pontecorvo, JETP 34, 247 (1958).

[17] B. Pontecorvo, JETP 53, 1717 (1967).

[18] H. V. . Klaptor-Kleingrothaus, neutrino '96, Procedings of the 17th International Conference on Neutrino Physics and Astrohysics, Helsinki, Finland, 1996, eds. K. Enqvist, K. Huiti and Maalampi, World Scientific, p317.

[19] T. Yanagida, O. Sawanda, and A. . Sugamoto, , in: Procedings Of The Workshop On The Unified Theory And The Baryon Number In The Universe, held at National Laboratory For High- Energy Phyiscs, February 13-14, 1979.

[20] R. N. Mohapatra and G. Senjanovic, Phys. Rev. D 23, 165 (1981).

[21] Z. Maki, M. Nakagawa, and S. Sakata, Prog. Theor. Phys 28, 870 (1962).

[22] L. Wolfenstein, Phys. Rev. D17, 2369 (1978).

[23] S. P. Mikheyev and A. Y. Smirnov, Sov. J. Nucl. Phys. 42, 1441 (1985).

[24] J. N. Bachall, http://www.sns.ias.edu/ jnb/.

[25] A. I. Abazov et al., Phys. Rev. Lett. 67, 3332 (1991).

[26] D. N. Abdurashitov et al., Phys. Rev. Lett. 77, 4708 (1996).

[27] P. Anselmann et al., Phys. Lett. B 285, 376 (1992).

[28] W. Hampel et al., Phys. Lett. B 388, 384 (1996).

[29] K. S. Hirata et al., Phys. Rev. Lett. 65, 1297 (1990).

[30] Y. Fukuda et al., Phys. Rev. Lett. 77, 1683 (1996).

[31] Y. Fukuda et al., Phys. Rev. Lett. 81, 1158 (1998). 
[32] Y. Fukuda et al., Phys. Rev. Lett. 82, 1810 (1999).

[33] The SNO Collaboration, NIM A 449, 172 (2000).

[34] Q. R. Ahmad et al., Phys. Rev. Lett. 89, 011301 (2002).

[35] Q. R. Ahmad et al., Phys. Rev. Lett. 89, 011302 (2002).

[36] R. Becker-Szendy et al., Phys. Rev. Lett. 69, 1010 (1992).

[37] R. Becker-Szendy et al., Nuc. Phys. Proc. Suppl. 38, 331 (1995).

[38] K. S. Hirata et al., Phys. Rev. Lett. B 280, 146 (1992).

[39] G. Battastoni et al., Nucl. Instr. Meth. A 245, 277 (1986).

[40] M. Aglietta et al., Europhys. Lett. 8, 611 (1989).

[41] K. Daum et al., Z. Phys. C 66, 417 (1995).

[42] W. W. M. Allison et al., Phys. Lett. B 449, 137 (1999).

[43] Y. Fukuda et al., Phys. Lett. B 433, 9 (1998).

[44] S. Fukuda et al., hep-ex/0009011 (2000).

[45] C. Yanagisawa, Nucl. Phys. Proc. Suppl. 95, 93 (2001).

[46] M. Apollonio et al., Phys. Lett. B 466, 415 (1998).

[47] K. Eguchi et al., Sumbmitted to Phys. Rev. Lett. B (2002).

[48] L. Ludovici et al., Nucl. Phys. Proc. Suppl. 91, 177 (2001).

[49] P. Astier et al., Phys. Lett. B. 611, 3 (2001).

[50] C. Athanassopoulus et al., Phys. Rev. Lett. 81, 1774 (1998).

[51] K. Nakamura, Nucl. Phys. Proc. Suppl. 91, 203 (2001). 
[52] K. Hagiwara et al., Physical Review D 66, 010001+ (2002).

[53] The MINOS Collaboration, NuMI Beam TDR, Technical Report, Fermilab, 1999.

[54] The MINOS Collaboration, MINOS Technical Design Report; FNAL internal document NuMI-L-337 (1998).

[55] J. Hylen et al., FERMILAB-TM-2018 (1997).

[56] V. Abramov et al., IFVE-96-91, IHEP-96-91 .

[57] P. Adamson, A. Para, and J. Thomas, nuMI-NOTE-SIM-0536; unpublished.

[58] O. Toker et al., Nucl. Instr. Meth. A 340, 572 (1994).

[59] IDE AS, Veritasveien 9, Box 315, N-1323 Hvik, Norway; www.ideas.no.

[60] T. Zimmerman and M. Sarraj, IEEE Trans. on Nuclear Science 43, 1683 (1996).

[61] C. Smith, Calibration of the MINOS Detectors and Extraction of Neutron Oscillation Parameters, D.Phil. Thesis, University of London (2002).

[62] S. E. K. Lang, J. Day et al., Nucl. Instr. Meth. A 461, 571 (2001).

[63] R. Nichols, Private communication.

[64] Nichia Corporation, 491 Oka, Kaminaka-Cho, Anan, Tokushima 774-8601, Japan; www.nichia.co.jp.

[65] Hewlett-Packard 3000 Hanover Street Palo Alto, CA 94304-1185, U.S.A.; www.hp.com.

[66] Bivar Inc; 4 Thomas, Irvine, CA 92618 , U.S.A.; www.bivar.com. 
[67] K. Ruddick, private communication. Data above $495 \mathrm{~nm}$ are from measurements and the University of Minnesota; the rest are from Bicron Corp. (using nominal doping).

[68] G. D. Description and S. Tool, CERN Program Library W5013, (1994).

[69] M. V. Mokhov and S. I. Stringanov, AIP Conf. Proc. 435, 543 (1997).

[70] M. Bonesini, A. Marchinonnia, F. Pietropaola, and T. T. de Fatis, hepph/0101163, .

[71] A. J. Malensek, Fermilab-FN 341, (1981).

[72] Barton et al., Phys. Rev. D. 27, 2580 (1983).

[73] Atherton et al., CERN 80-07, (1980).

[74] G. . Ambrosini, Euro. Phys. J. C10, 605 (1999).

[75] G. Collazuol et al., Report CERN-OPEN-98-032; Nucl. Instr. and Meth. A449, 609 (2000).

[76] N. Bozhko et al., Sov. J. Nucl. Phys. 29(3), 347 (1979).

[77] N. Bozhko et al., Sov. J. Nucl. Phys. 31(5), 664 (1980).

[78] N. Bozhko et al., Sov. J. Nucl. Phys. 31(6), 775 (1980).

[79] Andrzej Rybicki, Charged Hadron Production in Elementary and Nuclear Collisions at $158 \mathrm{Gev} / \mathrm{c}, \mathrm{Ph}$.D. Dissertation for the H. Niewodniczanski Institute of Nuclear Physics, Krakow, March 2002.

[80] O. Chvala, Extraction of Inclusive Cross Sections of Charged Pions Produced in Proton-Proton Collisions from the NA49 Experimental Data (2000).

[81] F. James and M. Roos, Computer Physics Communications 10, 343 (1975). 
[82] R. P. Feynman, Phys. Rev. Lett. 23, 1415 (1969). 